\title{
Size effects in epitaxial oxide thin films
}

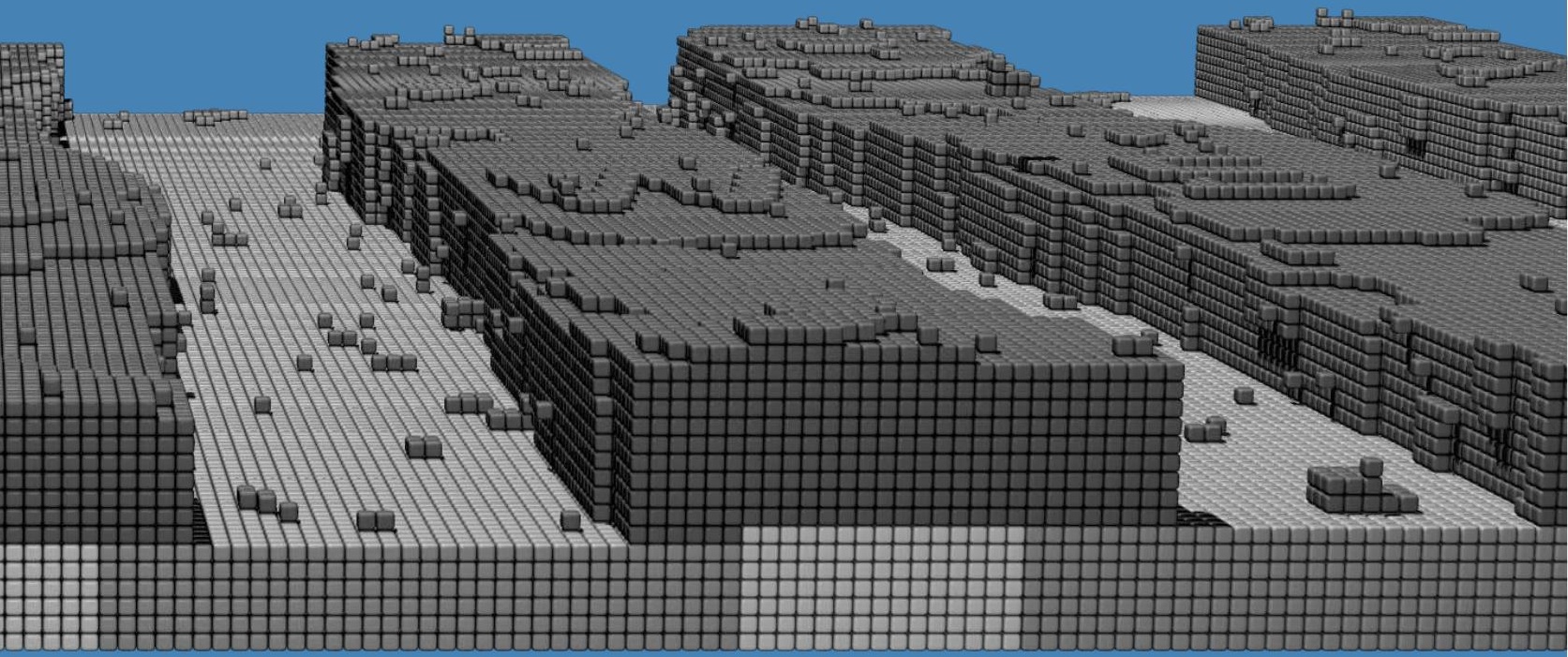




\section{Ph.D. committee}

\section{Chairman}

prof. dr. G. van der Steenhoven

\section{Secretary}

prof. dr. G. van der Steenhoven

\section{Supervisor}

prof. dr. ing. A.J.H.M. Rijnders

\section{Assistant-supervisor}

dr. ir. G. Koster

University of Twente

University of Twente

University of Twente

University of Twente

\section{Members}

prof. dr. ing. D.H.A. Blank

prof. dr. ir. H.J.W. Zandvliet

prof. dr. H.M. Christen

prof. dr. J. Aarts

prof. dr. B. Noheda
University of Twente

University of Twente

Oak Ridge National Laboratory

University of Leiden

University of Groningen

Cover Three dimensional impression of the formation of epitaxial nanowires on an ordered mixed terminated crystal surface. The light gray blocks at the bottom represent the different areas of surface mixed termination, e.g., DyO and $\mathrm{ScO}_{2}$ in the case of $\mathrm{DyScO}_{3}$. The dark blocks represent complete perovskite blocks of deposited film material, e.g., $\mathrm{SrRuO}_{3}$. This type of nanowire formation is described in chapters three, four and five of this thesis. The picture is generated using POVRay software and is based on actual Monte Carlo simulation results.

The research described in this thesis was carried out within the Inorganic Materials Science group, Department of Science and Technology and the MESA+ institute for Nanotechnology at the University of Twente. This work is financially supported by The Netherlands Organization for Scientific Research (NWO).

Size effects in epitaxial oxide thin films

Ph.D. Thesis, University of Twente

Printed by Gildeprint Drukkerijen

Copyright (c) 2014 by B. Kuiper

DOI: $10.3990 / 1.9789036536097$

ISBN: 978-90-365-3609-7

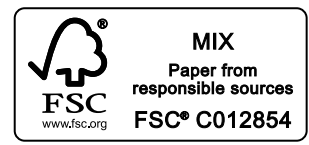




\title{
SIZE EFFECTS IN EPITAXIAL OXIDE THIN FILMS
}

\author{
PROEFSCHRIFT
}

ter verkrijging van

de graad van doctor aan de Universiteit Twente, op gezag van de rector magnificus,

prof. dr. H. Brinksma

volgens besluit van het College voor Promoties

in het openbaar te verdedigen

op donderdag 30 januari 2014 om 14:45 uur

door

Bouwe Kuiper

geboren op 17 oktober 1984

te Rotterdam 
Dit proefschrift is goedgekeurd door de promotor prof. dr. ing. A.J.H.M. Rijnders

en de assistent promotor

dr. ir. G. Koster 


\section{Contents}

1 Nanopatterned epitaxial oxide thin films 1

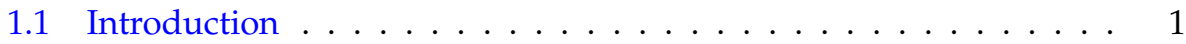

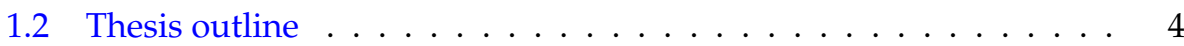

2 Fabrication and characterization of epitaxial oxide thin films 5

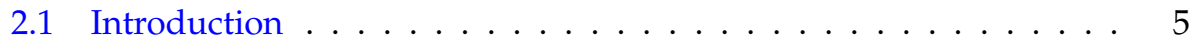

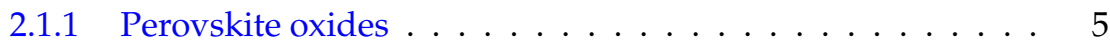

2.1.2 Epitaxy and strain $\ldots \ldots \ldots \ldots \ldots \ldots$

2.2 Thin film growth by pulsed laser deposition $\ldots \ldots \ldots \ldots$

2.2.1 Single crystal substrates and targets $\ldots \ldots \ldots \ldots$

2.2.2 Pulsed laser deposition parameters . . . . . . . . . . . 9

2.2.3 In situ reflection high energy electron diffraction . . . . . . . 11

2.3 Thin film characterization . . . . . . . . . . . . . . . . 12

2.3.1 Scanning probe/electron microscopy . . . . . . . . 12

$2.3 .2 \quad$ X-ray diffraction . . . . . . . . . . . . . . . . 13

2.3.3 Photoelectron spectroscopy . . . . . . . . . . . . . . . . 14

2.3.4 X-ray photoelectron diffraction . . . . . . . . . . 16

2.3.5 Magnetic and electrical characterization . . . . . . . . . . 20

2.4 Simulations and computer modeling . . . . . . . . . . . . . 21

2.4.1 Multiple scattering simulations . . . . . . . . . . . . . 21

2.4.2 Density functional theory calculations . . . . . . . . . . 21

2.4.3 Monte Carlo growth simulations . . . . . . . . . . . . . 21

$3 \mathrm{DyScO}_{3}(110)$ substrate surface termination control 25

3.1 Introduction . . . . . . . . . . . . . . 26

3.2 Mixed surface structure of annealed $\mathrm{DyScO}_{3} \ldots \ldots \ldots \ldots$

3.2.1 $\mathrm{DySCO}_{3}$ annealing for mixed termination . . . . . . . . . 29

3.2.2 Influence of the anneal parameters . . . . . . . . . . . . 31

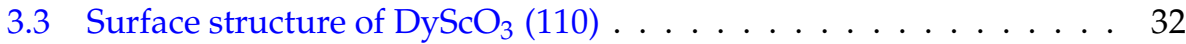

3.3.1 Selective wet chemical etching for $\mathrm{ScO}_{2}$ termination . . . . . 33

3.3.2 Structural characterization of $\mathrm{DyScO}_{3}(110)$ surfaces . . . . . 35

3.4 Artificially induced $\mathrm{DyO}$ termination $\ldots \ldots \ldots$. . . . . . . 39

3.5 Discussion . . . . . . . . . . . . . . . . . . . 41

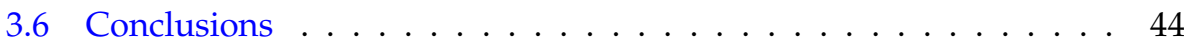


4 Self-organization on ordered oxide surface terminations 45

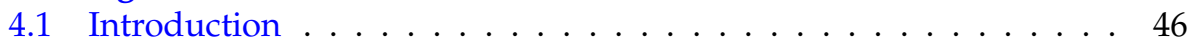

4.2 Self-organization and growth of $\mathrm{SrRuO}_{3} \ldots \ldots$. . . . . . . . . . . . . 47

4.2.1 Ordered mixed terminated $\mathrm{DyScO}_{3}$ substrates . . . . . . . . 48

4.2.2 $\mathrm{SrRuO}_{3}$ growth and $\mathrm{RHEED}$ analysis . . . . . . . . . . . . 48

4.2.3 Nanowire formation on $\mathrm{DyScO}_{3}$ substrates . . . . . . . . 50

4.2.4 Termination selective growth other substrates . . . . . . . 54

4.3 Structural and electronic characterization . . . . . . . . . . . . . . 54

4.3.1 Structural characterization . . . . . . . . . . . . . 54

4.3.2 Interface structure and configuration .......... 55

4.3 .3 Local electronic properties . . . . . . . . . . . . 56

4.4 Discussion . . . . . . . . . . . . . . . . 57

4.5 Conclusions ...................... 60

5 Growth kinetics on termination templates 61

5.1 Introduction . . . . . . . . . . . . . . . 62

5.1 .1 Atomistic view on thin film growth ......... 63

5.2 Monte Carlo type growth simulations . . . . . . . . . . . . . . 64

5.2.1 Three-dimensional kinetic Monte Carlo model . . . . . . . . 65

5.3 Growth simulations on termination templates . . . . . . . . . 67

5.3.1 Simulations on a simple termination template . . . . . . 68

5.3.2 Simulations on ordered termination templates . . . . . . . 69

5.3.3 Tuning simulated self-organization . . . . . . . . . 70

5.3.4 Time evolution of self-organized nanowire growth . . . . 70

5.3.5 Simulated growth on other templates . . . . . . . . 72

5.4 Discussion . . . . . . . . . . . . . . . . . 74

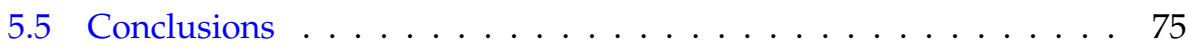

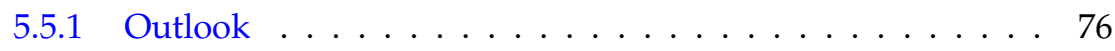

6 Structural phase transition in ultra-thin $\mathrm{SrCuO}_{2}$ films 77

6.1 Introduction . . . . . . . . . . . . . . . 78

6.2 Experimental results and simulations . . . . . . . . . . . 80

6.2.1 Substrate preparation and $\mathrm{SrCuO}_{2}$ film growth . . . . . . . . 80

6.2.2 $\mathrm{SrTiO}_{3}$ substrate reference structure . . . . . . . . . . . 81

6.2.3 $\mathrm{SrCuO}_{2}$ structural characterization by XPD . . . . . . . . . 84

6.2 .4 Thin film photoemission ............... 86

6.2.5 Quantitative photoemission ............. 88

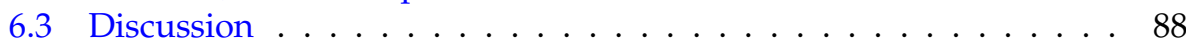

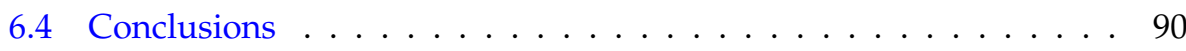

7 Role of interfacial oxygen octahedral coupling in strained $\mathrm{SrRuO}_{3}$ films 91

7.1 Introduction . . . . . . . . . . . . . . . 92

7.1.1 Sample geometry, orientation and structure . . . . . . . . 93

7.2 Critical thickness of strained $\mathrm{SrRuO}_{3} \ldots \ldots \ldots$. . . . . . . . . . . 94

7.2.1 Ground state of tetragonal $\mathrm{SrRuO}_{3} \ldots \ldots$. . . . . . . . 94 
7.2.2 Increased symmetry in ultra-thin films . . . . . . . . . 96

7.2 .3 Search for a possible AFM phase . . . . . . . . . . . . 97

7.2.4 Structural characterization by XPD . . . . . . . . . 98

7.3 Capping layer induced enhancement of ferromagnetism $\ldots \ldots 100$

$7.3 .1 \mathrm{~T}_{\mathrm{C}}$ enhancement . . . . . . . . . . . . . . 100

7.3.2 Selective etching of the $\mathrm{SrTiO}_{3}$ capping layer . . . . . . . . 104

7.4 Discussion . . . . . . . . . . . . . . . . . 105

7.4.1 Ground state of ultra-thin $\mathrm{SrRuO}_{3}$ films . . . . . . . . . 105

7.4.2 Capping induced $\mathrm{T}_{\mathrm{C}}$ enhancement . . . . . . . . . . 107

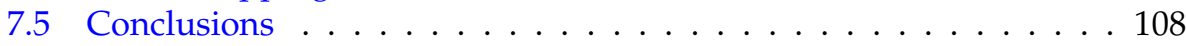

$\begin{array}{ll}\text { Bibliography } & 109\end{array}$

$\begin{array}{ll}\text { Summary } & 117\end{array}$

$\begin{array}{ll}\text { Samenvatting } & 121\end{array}$

$\begin{array}{ll}\text { Dankwoord } & 125\end{array}$ 


\section{Chapter 1}

\section{Nanopatterned epitaxial oxide thin films}

\subsection{Introduction}

Fabricating ever smaller devices which show ever more functionality is at the heart of modern day technological development in the field of electronics. This quest for smaller and more advanced electronics, requires the fabrication of components and structures with length-scales now reaching only several nanometers (a billionth of a meter). Material properties at the nanometer scale can be very different compared to their bulk counterparts. Volume to surface area ratios change and classical laws of physics cannot always be applied; quantum effects start to play a role. A famous quote from world-renowned physicist Feynman is often referred to in this regard:

"There is plenty of room at the bottom."

\section{Richard Feynman, December $1959^{[1]}$}

Although popular magazines discourage the use of this quote as introduction in scientific publications ${ }^{[2]}$ along with Moore's law, it perfectly summarizes the motivation for much of the research done in the field of nanotechnology in the past years and nicely fits the work done in this thesis.

While intrinsic (bulk) material properties may be lost or altered by size or quantum effects, new phenomena and properties can emerge, e.g., giant magnetoresistance, which is now commonly used in hard disk drives and sensor applications. ${ }^{[3,4]}$ The fabrication process of these small structures or thin films is usually different compared to conventional patterning techniques. New fabrication processes must be developed in order to create functional patterns at the nanometer scale and characterization techniques, like electron microscopy, should be improved to allow for analyzing the resulting structures and properties. In this respect, the fundamental material properties, the fabrication process and characterization methods are coupled and all require a great amount of study.

An interesting group of materials for both device fabrication and fundamental materials studies is the family of metal oxides. Within this group, the per- 
ovskite family contains a range of materials which all share a common oxygen backbone, with a multitude of different properties, e.g., magnetic, superconducting, ferro- and piezoelectric. By creating oxide heterostructures, several of these properties can be combined. The structure of this $\mathrm{ABO}_{3}$ perovskites subgroup, consists of alternating planes of $\mathrm{AO}$ and $\mathrm{BO}_{2}$ layers. Cations $\mathrm{A}$ and $\mathrm{B}$, either rare-earth or metal, are complemented by oxygen anions. The oxygen atoms form $\mathrm{BO}_{6}$ octahedra, which surround the B-site ions, as schematically indicated in Fig. 1.1(a). An intensively studied complex-oxide example is $\mathrm{YBa}_{2} \mathrm{Cu}_{3} \mathrm{O}_{6+x}$, a superconducting metal oxide, which was successfully fabricated using pulsed laser deposition (PLD). ${ }^{[5,6]}$ By artificial layering of a closely related compound, infinite-layer $\mathrm{SrCuO}_{2}$, a superconducting phase was prepared using PLD which was not observed in bulk. ${ }^{[7,8]}$ Infinite-layer $\mathrm{SrCuO}_{2}$ has a defective perovskite structure with one missing oxygen anion, as shown in Fig. 1.1(b).

The perovskite group of oxide materials contains a vast subgroup which is piezoelectric and/or ferroelectric. In this area, $\mathrm{Pb}(\mathrm{Zr}, \mathrm{Ti}) \mathrm{O}_{3}{ }^{[9]}$ is a commonly used material for creating functional devices, e.g., memory devices, actuators and sensors. Intriguing fundamental physical phenomena are also observed in this material class, for example in the $\mathrm{LaAlO}_{3}-\mathrm{SrTiO}_{3}{ }^{[10]}$ system. In this system, two wide-bandgap semiconductors are stacked on-top of each other, resulting in a conducting two-dimensional interface. The exact origin of this effect is still an active research topic.

Oxide heterostructures made of perovksite-type materials are usually prepared as two-dimensional layered sheets (thin films). The common oxygen backbone and crystal structure often allows for epitaxial growth, where the crystal structures of the film and substrate are aligned and coupled to each other at the interface $^{[11]}$, as sketched in Fig. 1.1(a). By taking advantage of epitaxy, the properties of thin films and nanostructures can be tuned or enhanced. For example, when straining commonly used $\mathrm{SrTiO}_{3}$ on a $\mathrm{DyScO}_{3}$ substrate, this normally dielectric material becomes ferroelectric. ${ }^{[12]}$ Recently, the role of the oxygen backbone and oxygen octahedral coupling across interfaces has attracted great interest as a new way to tune the properties of oxide thin films, apart from only considering substrate induced strain effect, e.g., $\mathrm{SrRuO}_{3}$ and $\mathrm{LaNiO}_{3} .{ }^{[13-15]}$

Next to the formation of two-dimensional layered sheets of material where the film thickness can be reduced to below one nanometer, as depicted in Fig. 1.1(c), lateral or in-plane control of material dimensions can be a useful way to tune material properties via size effects ${ }^{[16]}$, i.e., by making nanostructures with reduced dimensions. In this scenario, it is important to be able to distinguish intrinsic size effects from extrinsic factors, like surface roughening and structural changes at the surface. Although, these extrinsic effects might give rise to enhanced properties, in principal they are undesired. Therefore, a bottom-up approach for creating nanostructures is highly promising. For example, by using self-assembly or self-organization. ${ }^{[17]}$

In this thesis, perovskite-type thin films and nanostructures of different dimensionality, e.g., ribbons and thin films are fabricated using PLD and the effects of size reduction on the structure and properties studied in various material sys- 


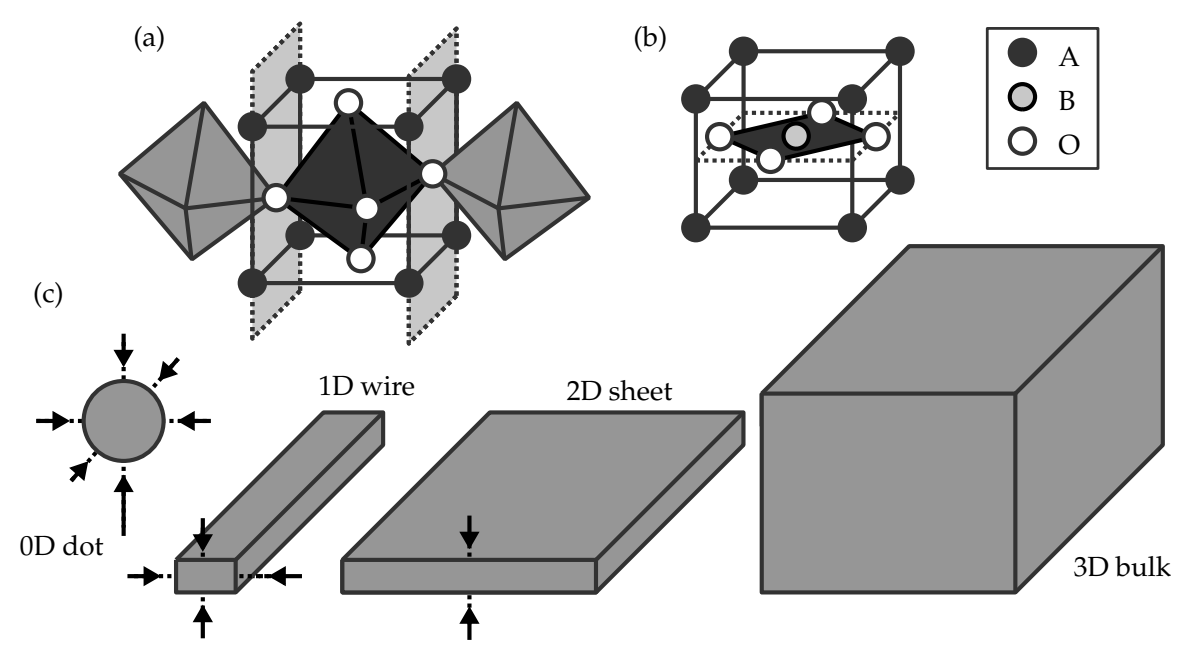

Figure 1.1: Schematic drawings of, (a) the oxygen backbone in perovskite-type oxides and the coupling of oxygen octahedra at interfaces (left and right sides of the cubic unitcell), (b) the infinite-layer, defective perovskite structure and (c) of structures with various dimensionalities. (0D) Zero-dimensional dot; all three spatial directions are reduced. (1D) One-dimensional wire or ribbon; two reduced directions. (2D) A two-dimensional thin film or sheet; one reduced direction and (3D) a bulk or three-dimensional object.

tems. By using epitaxial strain and tuning the interplay between oxygen octahedra across interfaces, the physical properties and structure of ultra-thin films are altered and enhanced. The performed experiments are aimed towards control of morphology, structure and physical properties by means of understanding the underlying physical mechanisms. Nanopatterns of $\mathrm{SrRuO}_{3}$ are created using a novel bottom-up technique, which relies on self-organization on insulating single-crystal templates. The resulting epitaxial structures are conducing, as $\mathrm{SrRuO}_{3}$ is one of the few conducting undoped complex oxides. The resulting electrode patterns can be used to study thin film size effects, where also the in-plane dimensions are reduced. The $\mathrm{DyScO}_{3}$ (110) starting templates for this type of growth are studied in detail, as they yield well-defined $\mathrm{SrRuO}_{3}$ nanopatterns. $\mathrm{DyScO}_{3}$ is of interest as a substrate material for epitaxial growth, due to its high crystalline quality. ${ }^{[18]}$ Moreover, $\mathrm{DyScO}_{3}$ can be used as a model system for a group of related scandate compounds, with a range of lattice constants. The $\mathrm{DyScO}_{3}(110)$ substrates, $\mathrm{SrRuO}_{3}$ nanowires and the atomic growth model of $\mathrm{SrRuO}_{3}$ on $\mathrm{DyScO}_{3}$ are studied and discussed in this thesis.

The effects of size reduction, epitaxial strain and the role of oxygen octahedral coupling are studied in two material systems. In thin films of $\mathrm{SrRuO}_{3}$, the influence of octahedral coupling and strain are studied both experimentally and theoretically, in order to tune and enhance the film properties. The oxygen sublattice structure of $\mathrm{SrRuO}_{3}$ is studied using various techniques, including X-ray photoelectron diffraction (XPD). The exact sensitivity of XPD to the oxygen sublattice 
is unknown. Therefore, $\mathrm{SrCuO}_{2}$ is studied as a model system for XPD analysis; a material system showing strong structural changes in the oxygen sublattice. Upon reducing the film thickness, in such $\mathrm{SrCuO}_{2}$ ultra-thin films, a theoretically predicted phase transformation, due to atomic rearrangement in the oxygen sublattice, is experimentally verified using XPD.

\subsection{Thesis outline}

This thesis contains five main scientific chapters and one chapter on sample fabrication/characterization techniques, chapter two. Chapters three to five discuss the fabrication of epitaxial oxide nanostructures on single crystal templates. The last two chapters, six and seven, are focused on material properties, where dimensions are reduced.

Chapter 3, describes the rare earth scandate surface structure, using $\mathrm{DyScO}_{3}$ (110) as a model system. The surface structure of singly terminated $\mathrm{DyScO}_{3}(110)$ and various other surface termination configurations are studied. By careful sample treatment, patterned surfaces with well-defined surface termination areas of $\mathrm{ScO}_{2}$ and $\mathrm{DyO}$ are created and their structure studied.

In chapter $4, \mathrm{DyScO}_{3}(110)$ surface termination templates are used as starting point for the fabrication of $\mathrm{SrRuO}_{3}$ nanostructures. Here doubly terminated $\mathrm{DySCO}_{3}$ substrates with a well-defined surface termination pattern are used to grow patterned thin films using PLD. $\mathrm{SrRuO}_{3}$ is sensitive to the local surface termination and self-organizes on the $\mathrm{ScO}_{2}$ terminated areas, leaving the DyO areas uncovered. The resulting nanostructures are characterized and their growth studied. The influence of the exact $\mathrm{DyScO}_{3}$ starting template is shown to be of great influence on the resulting $\mathrm{SrRuO}_{3}$ patterns.

In chapter 5 , a detailed growth model of these $\mathrm{SrRuO}_{3}$ patterns is provided. A kinetic Monte Carlo model is used to study diffusion, nucleation and growth of $\mathrm{SrRuO}_{3}$ on various surface termination templates. This model is based on a Solidon-Solid type two-dimensional growth model, but extended to allow for growth of wires and islands were atoms can also diffuse vertically. The time evolution of the model is compared to results found in chapter four and the model is used to study the mechanism driving the formation of the nanostructures.

In chapter 6, a thickness dependent phase transition in ultra-thin $\mathrm{SrCuO}_{2}$ films is revealed by XPD. A theoretically predicted phase transition from bulk planar $\mathrm{SrCuO}_{2}$ to chain-type $\mathrm{SrCuO}_{2}$ in ultra-thin films is confirmed. Moreover, the stoichiometry and electronic structure of both phases are determined.

Finally, in chapter 7, another thickness dependent transition is studied in $\mathrm{SrRuO}_{3}$ ultra-thin films. A change in magnetic and electrical properties is observed when the $\mathrm{SrRuO}_{3}$ thickness is reduced. Moreover, a capping layer is shown to be able to change the octahedral tilt and rotation angles, which allows for tuning of the ferromagnetic transition temperature. The experimental findings are supported by calculations. Initial experimental evidence for a possible structural transition in ultra-thin films below the critical thickness is discussed. 


\section{Chapter 2}

\section{Fabrication and characterization of epitaxial oxide thin films}

\subsection{Introduction}

Materials from the perovskite oxide crystal class with their rich variety of properties can be synthesized into epitaxial thin films using several deposition techniques, like sputtering deposition, molecular beam epitaxy and pulsed laser deposition (PLD). The latter technique, PLD, is used in this work, for the growth of thin films. The resulting film material properties and structural parameters are characterized, to reveal possible new or enhanced properties. In this chapter, an overview of the main experimental methods used in this thesis is provided. Special focus is directed towards relevant aspects of data analysis and newly developed features and methodologies specific for this thesis. For example, the use and development of an in situ X-ray photoelectron diffraction setup. In this chapter, first the structural properties of the relevant perovskite oxides and their thin film epitaxy are discussed, followed by the PLD growth process used for their synthesis. Finally, the data analysis, sample characterization techniques and Monte Carlo simulations used in this work are discussed.

\subsubsection{Perovskite oxides}

The perovskite crystal class describes a family of compounds, which all have a structure similar to $\mathrm{CaTiO}_{3}$, with a general formula $\mathrm{ABO}_{3} \cdot \mathrm{CaTiO}_{3}$ was discovered in 1839 and named after Lev Perovski. In a perovskite type crystal, cations $A$ and B of different size, are usually rare-earth or transition metals which are accompanied by oxygen anions. In the ideal cubic case the structure can be described by a network of corner-sharing oxygen $\mathrm{BO}_{6}$ octahedra, with oxygen atoms at the faces of the unit-cell, A-site cations at the corner positions and the B-site cation at the center. In this structure, the B-site cation is 6-fold coordinated and the A-site atom has a 12-fold coordination. The most common perovskites are slightly distorted from the ideal cubic shape due to a non ideal relative ratio between A and B ion sizes, resulting in tetragonal or orthorhombic crystal struc- 


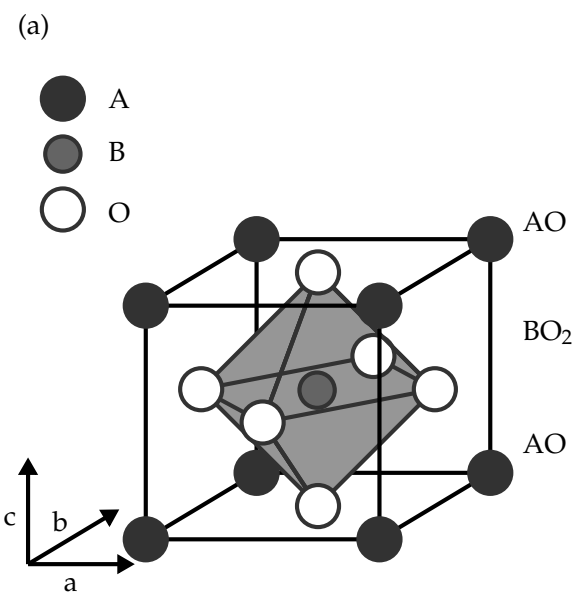

(b)

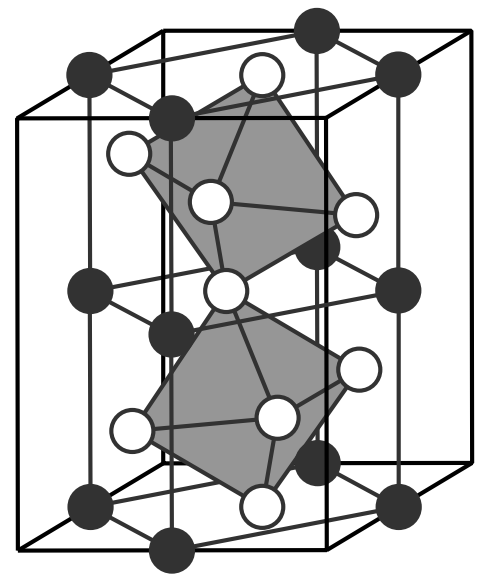

Figure 2.1: Schematic drawings of perovskite-type crystal structures, (a) A cubic $\mathrm{ABO}_{3}$ crystal structure, which is intrinsically layered in the [001] direction, showing alternating planes of $\mathrm{AO}$ and $\mathrm{BO}_{2}$. (b) Orthorhombic structure, which has a $\sqrt{(2)} \times \sqrt{(2)} \times 2$ times larger unit-cell and thus contains the equivalent of four pseudo-cubic unit-cells. Two pseudo-cubic unit-cells are drawn in (b), here the (110) plane is often used for substrates.

tures. ${ }^{1}$ In such a system the $\mathrm{BO}_{6}$ octahedra tilt or rotate, resulting in a lowering of the symmetry. Therefore, a larger unit-cell definition is required. However, it is possible to define a psuedo-cubic unit-cell within most perovskites, where the symmetry reduction is ignored. In general, this simplification allows for easy comparison and discussion of strain effects of various perovskites, independent of their exact structure. Schematic drawings of a cubic and an orthorhombic perovskite structure are shown in Fig. 2.1, two pseudo-cubic sub-unit-cells are drawn within the orthorhombic structure, in Fig. 2.1(b).

Not all perovskites can be readily synthesized as single crystals. However, using a process like PLD, it is possible to synthesize many types of perovskite thin films on single crystal substrate templates. In Table 2.1 an overview of the perovskite type materials used in this thesis is shown. Most of the materials in the list can be created in bulk crystal form and used as a substrate. However, for example $\mathrm{SrRuO}_{3}$ is difficult to prepare in bulk single crystal form, but can be prepared as crystalline thin film. Materials only used as thin film are labeled $\left(^{*}\right)$ in Table 2.1. However, for example $\mathrm{SrTiO}_{3}$ is used as substrate and as thin film capping layer.

\footnotetext{
${ }^{1}$ The Goldschmidt tolerance factor can used to describe how much the ion radii ratio deviates from an ideal cubic scenario. It is effectively an indicator of the stability and distortions of crystal structures. ${ }^{[19]}$
} 
Table 2.1: Overview of substrate and film materials used or discussed in this thesis. Their (pseudo)-cubic lattice constant $\left(0.5 \sqrt{a^{2}+b^{2}}\right)$ is given for non-cubic crystals. Materials marked [*] are only prepared as a thin film, all other materials are discussed or used as a substrate, but can also be prepared as a thin film. ${ }^{[8,20-22]}$

\begin{tabular}{cccc}
\hline \hline Material & a $p c(\AA)$ & crystal structure & orientation \\
\hline $\mathrm{SrTiO}_{3}$ & 3.905 & cubic & $(001)$ \\
$\mathrm{DyScO}_{3}$ & 3.95 & orthorhombic & $(110)$ \\
$\mathrm{NdGaO}_{3}$ & 3.86 & orthorhombic & $(110)$ \\
$\mathrm{LaAlO}_{3}$ & 3.89 & cubic & $(001)$ \\
$\mathrm{LSAT}^{*}$ & 3.87 & cubic & $(001)$ \\
$\mathrm{SrRuO}_{3}{ }^{*}$ & 3.93 & orthorhombic & $(110)$ \\
$\mathrm{SrCuO}_{2}$ & $3.4 / 3.8$ & tetragonal & $(001)$ \\
\hline \hline
\end{tabular}

\subsubsection{Epitaxy and strain}

Perovskite crystals with different unit-cell parameters can be stacked on-top of each other in heterostructures. In case of an epitaxial ${ }^{2}$ thin film grown on a single crystal substrate, the orientation of the film is well-defined with respect to the substrate. ${ }^{[23]}$ In case of homoepitaxy, the crystal structure of the film and substrate are lattice matched. In heteroepitaxy, the lattice parameters are unmatched. When the lattice mismatch is small, the film can be strained and to the substrate, called stained epitaxy. In Fig. 2.2 two examples of such strained films are given, for (a) compressive strain and (b) tensile strain. Alternatively, a film can relax towards its bulk values, but still show a well-defined orientation with respect to the substrate, called relaxed epitaxy. In this thesis all films are heteroepitaxial and the term epitaxy refers to strained epitaxy, i.e., all films are fully strained.

When a perovskite crystal is strained its structure will be deformed. These deformations will alter the crystal structure and affect the $\mathrm{BO}_{6}$ octahedra. Possible alterations of the octahedra are depicted in Fig. 2.2, where for example the octahedra deform or tilt/rotation angles are changed. Changes in the octahedral tilt pattern without deformations can be classified using Glazer notation. ${ }^{[24]}$ In this notation the magnitude and symmetry of the octahedral tilts are noted for all three spatial directions for a $2 \times 2 \times 2$ pseudo-cubic structure, e.g., a single symmetric rotation around the c-axis is noted as $\mathrm{a}^{0} \mathrm{a}^{0} \mathrm{c}^{+}$, which corresponds to a tetragonal structure (space group \#127).

At the interface between two perovskite oxides these tilt patterns should be matched, as they are shared among both layers. This coupling provides an additional parameter, which can influence the tilt angles and possible deformations of the octahedra near interfaces. For example, the schematic of octahedra rotations near the substrate-film interface in Fig. 2.2(b), does not have a continuous octahedra pattern. This is physically not possible and has to be resolved, for example, by deformations of the octahedra in the substrate or film.

\footnotetext{
${ }^{2}$ The term epitaxy comes from the Greek roots epi and taxis, meaning above and in an ordered manner.
} 


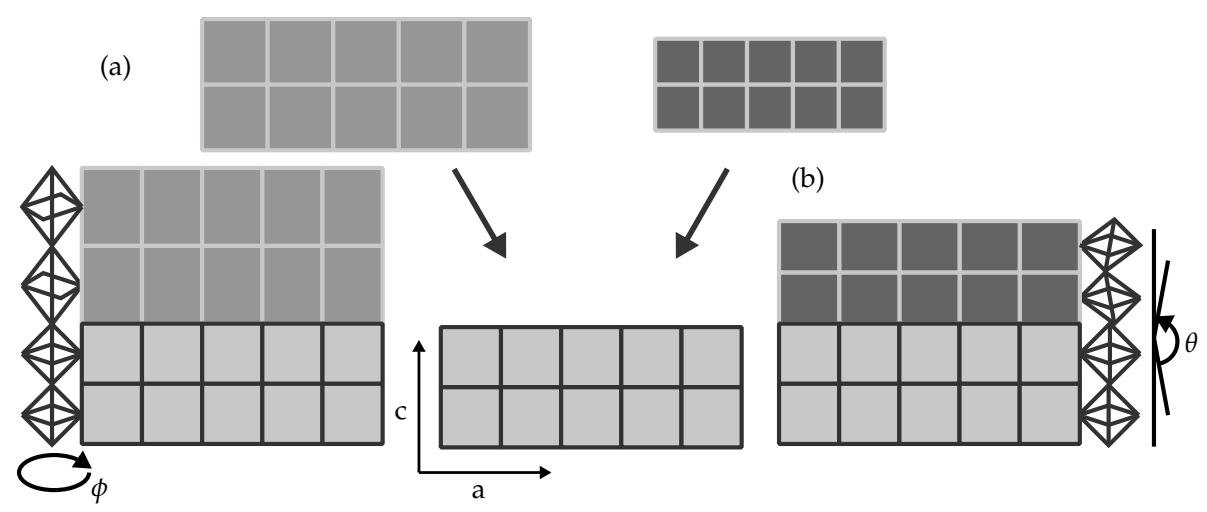

Figure 2.2: Schematic drawings of strained epitaxial thin films, (a) compressive where the film lattice-constant is larger than that of the film and (b) tensile strain in the opposite case. Artist impressions of the effect of strain of the oxygen $\mathrm{BO}_{6}$ octahedra are drawn in both scenarios, deformations of an ideal perfect cubic perovskite structure can result in tilting, rotation and deformations in the octahedra.

\subsection{Thin film growth by pulsed laser deposition}

Oxide thin films used in this work are made using pulsed laser deposition (PLD). In PLD, a high intensity $\mathrm{KrF}$ excimer laser is used to ablate material within a vacuum chamber. A plasma is formed, which expands and is collected on a single crystal substrate. The substrate temperature is controlled along with other deposition parameters, like laser fluence, laser spot-size, target-substrate distance and background gas mixture. By optimizing these settings, a high quality epitaxial thin film can be fabricated. A schematic of the PLD setup is given in Fig. 2.3(a).

PLD allows for the deposition of a vast group of oxide materials. Moreover, materials containing volatile species like $\mathrm{Pb}, \mathrm{Bi}$ and $\mathrm{Ru}$ can be deposited with a stoichiometric composition, most likely linked to the high supersaturation in PLD. The high supersaturation gives rise to a relatively high nucleation density and step density. By subsequent deposition from different targets, artificial stacks or heterostructures can be made.

During growth the film structure can be monitored using in situ reflection high energy electron diffraction (RHEED). Using RHEED it is possible to monitor the growth rate and gather information on the films surface morphology and structure. Moreover, by using RHEED during PLD, growth control on an atomic scale is possible.

\subsubsection{Single crystal substrates and targets}

Single crystal substrates: $\mathrm{SrTiO}_{3}(001), \mathrm{DyScO}_{3}$ (110), $\mathrm{NdGaO}_{3}$ (110) and 0.05 wt.\% Nb doped $\mathrm{SrTiO}_{3}$ (001) were supplied by Crystec $\mathrm{GmbH}$, Germany with a typical size of $5 \times 5 \times 0.5 \mathrm{~mm}^{3}$ and a miscut angle with respect to the desired crys- 
tal plane below $0.2^{\circ}$. Prior to annealing, the substrates were visually inspected for surface contaminations using an optical microscope. When required, substrates were cleaned using acetone and ethanol under ultra-sonic agitation.

The as-received substrates were annealed in a tube furnace to achieve ordered terrace steps. Annealing of $\mathrm{DyScO}_{3}$ and $\mathrm{NdGaO}_{3}$ was done at $1000{ }^{\circ} \mathrm{C}$ under an oxygen flow of $150 \mathrm{ml} / \mathrm{min}$. The anneal time was varied between 15 minutes and several days. $\mathrm{SrTiO}_{3}$ was annealed at $950{ }^{\circ} \mathrm{C}$ for 90 minutes under oxygen flow.

$\mathrm{SrTiO}_{3}$ substrates were chemically treated to obtain a single $\mathrm{TiO}_{2}$ surface termination. ${ }^{25]}$ Selective wet chemical etching in order to achieve single termination on $\mathrm{DyScO}_{3}$ substrates was done using a $12 \mathrm{M}$ and $1 \mathrm{M} \mathrm{NaOH}(\mathrm{aq})$ solution prepared using deionized water in an ultrasonic bath for at least 1 hours per step. ${ }^{[26]}$ Prior to selective etching using $\mathrm{NaOH}$, a surface roughening step was applied to some samples. The surface roughening was done using buffered HF $\left(\mathrm{NH}_{4} \mathrm{~F}: \mathrm{HF}=87.5: 12.5, \mathrm{pH}=5.5\right)$ for 30-60 seconds in an ultrasonic bath.

The samples were glued to sample plates for the deposition using Leitsilber glue (silver paint). Upon loading the substrates into vacuum they were heated to $\sim 150{ }^{\circ} \mathrm{C}$ in order to cure the glue and clean the sample surface.

Single crystal $\mathrm{SrRuO}_{3}$ and $\mathrm{SrCuO}_{2}$ targets were not available. Therefore, compressed powder pellets supplied by Praxair were used for PLD of $\mathrm{SrRuO}_{3}$ and $\mathrm{SrCuO}_{2}$. The area of the target used for material ablation was sand grinded and pre-ablated prior to each deposition. Circular targets with a diameter of $2.5 \mathrm{~cm}$ were typically used. The excimer laser beam was scanned over the target surface during ablation.

\subsubsection{Pulsed laser deposition parameters}

A PLD system from Twente Solid State Technology BV, was used in a cluster setup. This cluster setup allowed for sample fabrication and analysis in ultra high vacuum (UHV). The PLD was connected via a central storage chamber to an Omicron Nanotechnolgy $\mathrm{GmbH}$ (Oxford instruments) scanning probe microscope (SPM) and an Omicron surface analysis chamber. A $248 \mathrm{~nm}$ excimer laser with a pulse duration of 25 ns was used to ablate material from sintered power or single crystal targets. A rectangular mask was used to create a well-defined and homogeneous laser profile on the target. The laser fluence was controlled using a variable beam attenuator. The background gas pressure was in the range of $10^{-8}$ mbar. During deposition the surface structure was monitored using RHEED, at pressures up to 0.3 mbar, this is possible due to a differential pumping stage. ${ }^{[6]}$ A Staib instruments RHEED setup was used for the work presented in this thesis, operated at $30 \mathrm{keV}$.

Sample heating was done using a resistive heater or using a newly developed infrared laser heating system. The resistive heater allowed for accurate temperature control up to $\sim 850{ }^{\circ} \mathrm{C}$ in this setup. In order to increase the maximum temperature and allow for fast temperature modulation, a $120 \mathrm{~W}$ Coherent Quattro FAP laser was used for heating the sample plates directly. The maximum sample temperature was increased to $1100{ }^{\circ} \mathrm{C}$. Using this heating method, it was possible 
(a)

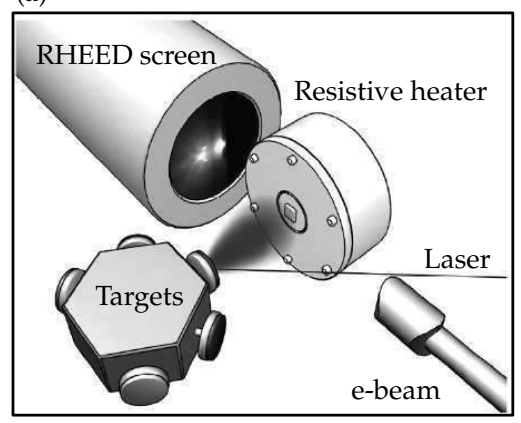

(b)

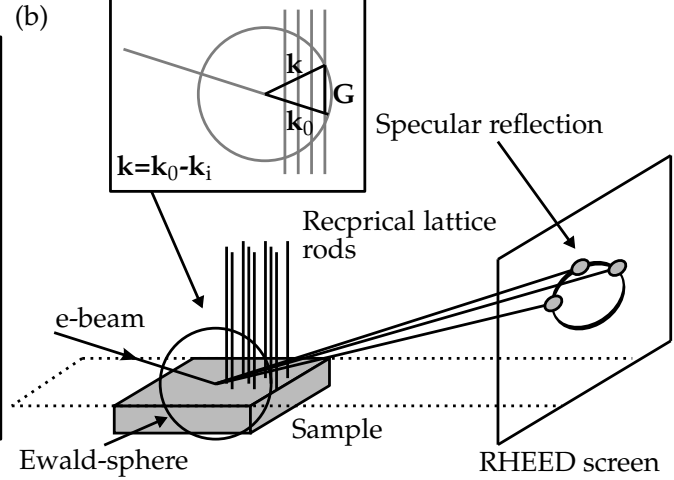

Figure 2.3: Schematic drawing of pulsed laser deposition (PLD) in (a) and reflection high energy electron diffraction (RHEED) in (b). In the PLD setup, the target carousel, resistive heater with a substrate, laser-beam, RHEED electron gun and RHEED camera are depicted. The resistive heater can be exchanged with an infrared laser-heating sample holder. The RHEED schematic gives an impression on how the observed diffraction pattern on the RHEED screen is obtained. Whenever the Ewald sphere intersects a reciprocal lattice rod and the diffraction conditions are met, an intensity maximum is observed next to the specular reflection. Image (a) is adapted from Huijben ${ }^{[27]}$.

to quickly change the sample temperature during deposition, up to $\sim 25^{\circ} \mathrm{C} / \mathrm{sec}$. The spot-size of the infrared laser was about $1 \mathrm{~cm}^{2}$ and aligned on the backside of the sample plate prior to every deposition. The sample-plate temperature was measured using an infrared thermometer, for good laser adsorption and close to black-body radiation the back of the sample plate was roughened and oxidized.

\section{$\mathrm{SrRuO}_{3}$ growth parameters}

$\mathrm{SrRuO}_{3}$ growth by PLD was done at an energy density of $2.1 \mathrm{~J} / \mathrm{cm}^{2}$, a laser repetition rate of $1 \mathrm{~Hz}$ and a spotsize of $2.3 \mathrm{~mm}^{2} . \mathrm{SrRuO}_{3}$ was ablated from a compressed powder pellet with a target-substrate distance of $5 \mathrm{~cm}$. The background pressure was set to 0.3 mbar with a gas mixture consisting of equal parts oxygen and argon. The substrate temperature was set between 600 and $700{ }^{\circ} \mathrm{C}$. Samples were post-annealed during cool-down at a rate of $25 \%$ min in $100 \mathrm{mbar}$ of oxygen.

\section{$\mathrm{SrTiO}_{3}$ growth parameters}

$\mathrm{SrTiO}_{3}$ films grown as capping-layers on-top of $\mathrm{SrRuO}_{3}$ were grown at the same settings as $\mathrm{SrRuO}_{3}$, except for the laser fluence and background pressure. The laser fluence was set to $1.3 \mathrm{~J} / \mathrm{cm}^{2}$ and a background gas consisting of $0.01 \mathrm{mbar}$ oxygen was used to grow $\mathrm{SrTiO}_{3}$. 


\section{$\mathrm{SrCuO}_{2}$ growth parameters}

$\mathrm{SrCuO}_{2}$ thin films were grown using a laser fluence of $2.0 \mathrm{~J} / \mathrm{cm}^{2}$, a spotsize of $1.8 \mathrm{~mm}^{2}$, laser repetition rate of $1 \mathrm{~Hz}$, substrate temperature of $650{ }^{\circ} \mathrm{C}$, target substrate distance of $5 \mathrm{~cm}$ and a pressure of 0.3 mbar oxygen. The target used is oxygen rich, $\mathrm{SrCuO}_{2.5}$. The samples were cooled down at a rate of $10^{\circ} / \mathrm{min}$ in the growth pressure.

\subsubsection{In situ reflection high energy electron diffraction}

In situ reflection high energy electron diffraction (RHEED) can be done during PLD, due to the gracing incidence and emission angles of the electron beam. The setup does not block the PLD plasma from reaching the sample surface. A schematic of the RHEED setup and main working principle is shown in Fig. 2.3(b). The electron beam is focused on a substrate, under a gracing angle and a photoluminescent screen in combination with a CCD camera was used to measure the intensity of reflected electrons.

The electrons are reflected of the sample surface. The crystal structure of the surface gives rise to diffraction peaks on the RHEED screen. The observed diffraction pattern depends on the crystal structure of the top surface. ${ }^{[28,29]}$ The pattern can be calculated based on the reciprocal lattice structure. In three dimensions, a reciprocal lattice consists of points. However, the reciprocal lattice of a twodimensional crystal is not represented by points, but by lines or rods, due to the dimension reduction. These rods intersect the reciprocal lattice points of a similar bulk crystal. Diffraction conditions are satisfied where these rods of reciprocal lattice intersect the so-called Ewald sphere. The Ewald sphere construction relates the wavevector of the incident electron beam with the diffraction conditions of a crystal lattice.

The relationship $\vec{k}=\overrightarrow{k_{0}}-\overrightarrow{k_{i}}$ defines the scattering wave vector $\vec{k}$ as a function of the wavevector of the incident beam $\overrightarrow{k_{0}}$ and the wavevector at any intersection between the Ewald sphere and the reciprocal lattice. Where, $\overrightarrow{k_{0}}=2 \pi / \lambda$ with $\lambda$ being the electron wavelength. $\vec{k}$ is related to the crystal plane spacing. The peak which is found at an take-of angle equal to the incidence angle is called the specular reflection. Many of the reciprocal rods meet the diffraction conditions. Only a selected few will give rise to a peaks on the RHEED screen, due to the gracing incidence angle of electron beam used in the setup. Intersections of the reciprocal lattice rods with the Ewald sphere lie on concentric circles, called the Laue circles. Therefore, the spots on the RHEED screen are also observed on circular shapes. The azimuthal angle of the sample with respect to the Laue circle is aligned in such a way that a circular pattern is observed perpendicular to the sample surface, i.e., perpendicular to a certain atomic row. Such atomic rows are labeled with their corresponding in-plane $[h k]$ values.

When the crystal surface is rough, transmission of electrons will yield additional diffraction peaks, which are three-dimensional in nature, in contrast to the above described two-dimensional effect. Such three-dimensional patterns do not 
show a clear dependence of azimuthal and polar rotations of the sample with respect to the incident electron beam. If only two-dimensional spots are observed, the sample can be considered atomically smooth and the diffraction pattern is related to the in-plane surface crystal structure of the sample.

The time-evolution of the intensity of the specular spot can be recorded during PLD growth. The intensity scales with the coverage of the sample; the highest intensity is found for complete coverage. During heteroepitaxy the intensity of the RHEED pattern is also influenced by the type of scattering atoms and their scattering cross-sections. An alternative to monitoring the intensity of the specular spot to study the formation of layered thin films is to extract the full-widthhalf-maximum (FWHM) of the peak shape. The FWHM roughly scales inversely with the intensity of the specular spot, since for partial coverage the observed scattering is slightly more diffuse compared to full coverage, resulting in a larger FWHM.

\subsection{Thin film characterization}

Before and after growth, the sample surface morphologies, structures and properties were studied using various techniques. In this section, first the sample morphology analysis and local conductivity analysis using scanning probe techniques is discussed. Next, the samples structural analysis methods are shown, as for example X-ray diffraction. Finally, the electronic and magnetic characterization methods are discussed.

\subsubsection{Scanning probe/electron microscopy}

\section{Scanning probe microscopy}

Sample surface morphologies were measured using various scanning probe microscopy (SPM) techniques. STM experiments were done using an Omicron nanotechnology $\mathrm{GmbH}$ (Oxford Instruments) variable temperature SPM in UHV conditions. AFM was performed in this thesis using the Omicron SPM setup and two ex situ systems, a Bruker ICON Dimension AFM and a NanoScope III AFM.

Height information is obtained using AFM by monitoring the position of laserspot which is reflected of the top of the tip-cantilever. AFM can be performed in several operation modes, the most intuitive one being contact-mode $(\mathrm{CM})$, where the tip is in close contact with the sample surface and the interaction strength is large. CM-AFM allows for simulations recording of friction force images. Friction force contrast is obtained when scanning a tip perpendicular to its cantilever orientation, as a result of the scanning movement the tip might bend due to sample induced drag. This bending will result in a deflection of the laser beam and is a measure of the tip-surface interaction strength. Both ex situ AFM machines could also be used in tapping mode (TM), where the tip is oscillated close to its resonance frequency. The amplitude of the oscillation is reduced when the tip approaches the sample surface. Moreover, the phase signal is a measure of the 
samples elasticity, adhesion and friction interactions with the tip. In effect, this provides similar information as CM-AFM friction contrast. The in situ AFM was operated mainly in non-contact (NC) mode, but could also be used in contactmode. In NC-AFM the interaction strength between the tip and sample is reduced with respect to TM-AFM and CM-AFM, but is in principle comparable to TM-AFM when operated in amplitude modulation mode. Local conductivity measurements were done using the Bruker ICON Dimension AFM, with the tunneling AFM (TUNA) module.

\section{Electron microscopy}

Scanning electron microscope (SEM) images of samples surfaces and nanostructures were recorded using a JSM-6490 high vacuum SEM (Jeol; Tokyo, Japan). Detailed structural characterization was done using transmission electron microscopy $(\mathrm{TEM})^{3}$ experiments on a FEI Titan 3 microscope operated at $120 \mathrm{kV} .^{[30]}$. Scanning TEM (STEM), electron energy loss spectroscopy (EELS) and high-angle annular dark-field imaging (HAADF) or Z-contrast modes of the TEM are used in this work.

\subsubsection{X-ray diffraction}

X-ray diffraction (XRD) experiments were done using a Bruker GmbH (Germany) D8 Discover diffractometer. Moreover, in-plane (XRD) ${ }^{4}$ experiments were done at beamline 7-2 of the Stanford Synchrotron Radiation Laboratory at SLAC (USA).

\section{Surface X-ray diffraction}

Surface X-ray diffraction (SXRD) is a highly surface sensitive technique, which can be used to determine the structure of crystal surfaces by measuring the intensity profile of crystal truncation rods (CTR). ${ }^{[31]}$ A CTR is the result the twodimensional nature of a surface layer, which leads to scattering intensity in between bulk Bragg peaks in a direction perpendicular to surface, i.e., the CTRs are lines in reciprocal space as compared to points normally observed for a bulk crystal. SXRD ${ }^{5}$ measurements were done using the $(2+3)$ axis diffractometer on beamline BM26 (DUBBLE) at ESRF synchrotron in Grenoble, France operated at $16 \mathrm{keV} .{ }^{[32]}$ Measurements were done under an nitrogen flow at $250{ }^{\circ} \mathrm{C}$ to limit water adsorption. At least six crystal truncation rods and the specular rod were measured for each sample. The data was analyzed with the ANA-ROD package ${ }^{[33]}$ using $\chi^{2}$ as goodness-of-fit criterion.

\footnotetext{
${ }^{3}$ TEM experiments performed by Ricardo Egavil, EMAT, University of Antwerp, Belgium (2012). TEM samples were prepared in collaboration with Brian Smith, University of Twente, Enschede (2012).

${ }^{4}$ XRD synchrotron experiments done in collaboration with Arturas Vailionis, Geballe Laboratory for Advanced Materials in Stanford (USA) (2012)

${ }^{5}$ SXRD experiments were performed in collaboration with Paul Tinnemans from the Radboud University Nijmegen, The Netherlands, Sybolt Harkema from the University of Twente, Enschede, The Netherlands and Guiseppe Portale from ESRF, Grenoble, France.
} 


\subsubsection{Photoelectron spectroscopy}

Thin film stoichiometry and electronic structure were studied using X-ray and ultraviolet photoemission spectroscopy, (XPS) and (UPS). In this XPS setup, the sample could be rotated and tilted, which allowed for doing angle dependent (diffraction) measurements, called X-ray photoelectron diffraction (XPD). This specific XPD setup was not used before, to perform such experiments. Therefore, below a short introduction into XPS and XPD is provided and the relevant data analysis and background removal methods briefly discussed.

\section{Experimental setup}

XPS and UPS were performed in situ on an Omicron nanotechnology $\mathrm{GmbH}$ (Oxford Instruments) Surface Analysis system, with a background pressure of $5 \times 10^{-11}$ mbar. Measurements were done using a monochromatic Al K $\alpha$ X-ray source (XM1000) with a kinetic energy of $1486.7 \mathrm{eV}$, and analyzed using a 7 channel EA 125 electron analyzer operated in CAE mode. For the XPD experiments, the acceptance angle of the detector was set to 4 degrees by using medium magnification and an entrance slit of $6 \times 12 \mathrm{~mm}^{2}$ was used, resulting in an analysis area of $3 \times 6 \mathrm{~mm}^{2}$. A Thermionics 5 axis sample stage was used for rotating the sample for XPD measurements. The pass energy was set to $100 \mathrm{eV}$ for XPD measurements. The $[001]_{p c}$ peak at zero degrees was used to correct for sample alignment errors due to deviations in gluing the sample to the sample holder.

A schematic of the XPD setup is shown in Fig. 2.4(a). A rotating sample geometry was used, where the azimuthal angle $(\phi)$ and the polar angle $(\theta)$ could be adjusted. The electron yield was recorded as a function of the kinetic energy, polar and azimuthal emission angles. The angle between the $X$-ray source and electron analyzer $(\beta)$ was fixed at $81^{\circ}$. For map scans were recorded using azimuthal steps of $2-3^{\circ}$ and polar steps of $1^{\circ}$. High resolution $\theta$-scans were recorded with step-sizes down to $0.1^{\circ}$.

\section{X-ray photoelectron spectroscopy}

Spectroscopic analysis of X-ray generated electrons via the photoelectric effect, called photoelectrons, is a well-known technique for chemical analysis of solids. ${ }^{[34]}$ By accurately measuring the kinetic energy of the electrons emitted from the sample, one can determine the core level binding energies, which are related to the electronic structure of the sample. A schematic representation of energy diagram is given in Fig. 2.4(b) and the relationship the kinetic energy $E_{K}$, photon source energy $h v$ and the binding energy for conducting samples is given by Eq. (2.1), where $\phi_{s p}$ is the work function of the detector.

$$
E_{K}=h v-E_{B}^{F}-\phi_{s p}
$$

If the work function of the spectrometer is known and the sample is metallic, i.e., the Fermi levels of the sample and the spectrometer are aligned, than the work 
function of the sample does not appear in the equation. ${ }^{6}$ For insulating samples a common reference is required, due to sample charging. For example the position of a common peak from the substrate or a line from a common surface contaminant, like C 1s, can be used to correct for shifts in the observed kinetic energy due to sample charging.

XPS is a surface sensitive technique, due to strong attenuation of electrons in solid material. This electronic attenuation depends on several factors like the experimental geometry and electron transport in the solid, but can be estimated using the inelastic mean free path (IMFP) and Beer-Lambert law, Eq. (2.2). The measured intensity $I_{z}$ is equal to $I_{0}$ attenuated exponentially depending on the IMFP $\lambda$ and the electron take-off-angle $\theta$ to the plane of the sample. Typical sampling depths for $\mathrm{O} 1$ s photoelectrons using $\mathrm{Al} \mathrm{K} \alpha$ radiation at normal incidence are in the order of a few nanometers. ${ }^{[34]}$

$$
I_{z}=I_{0} \cdot \exp \left(\frac{-z}{\lambda \sin (\theta)}\right)
$$

Next to elastic single photon-electron interactions, also various other electron generation paths are possible. Like the generation of inelastically scattered electrons, called secondary electrons. These secondary electrons give rise to a background in the spectrum, but also contain information about the sample. Moreover, two electron process can give rise to Auger electrons, where a valence electron fills up a deeper lying core hole releasing enough energy to emit another valence electron. Other processes which determine the peak shape and fine structure are for example, multiplet splitting, shake-up satellites and plasmon loss peaks. ${ }^{[34]}$

\section{XPS background removal}

Selection of the background type and peak shape functions for analyzing photoemission spectra is of influence to quantitative measurements. There are different background types available, each with its own specific drawbacks and strengths. The simplest background type is a linear fit, but a popular general purpose background choice is the Shirley background. Both the linear and Shirley background are purely mathematical and have no physical origin. For fitting peak shapes a Voigt ${ }^{[35]}$ function is often used, a combination of a Gaussian and Lorentzian line shape. The Gaussian part represents the instrumental and thermal broadening and the Lorentzian part the life-time broadening effects. In this thesis, linear backgrounds are used for XPD spectra, as they are easy to compute for all spectra automatically. Shirley backgrounds are used for XPS spectra. For fitting peak shapes in XPS spectra, either Gaussian or Voigt functions are used.

\footnotetext{
${ }^{6}$ The work function of the spectrometer was calibrated using a Au reference sample.
} 
(a)

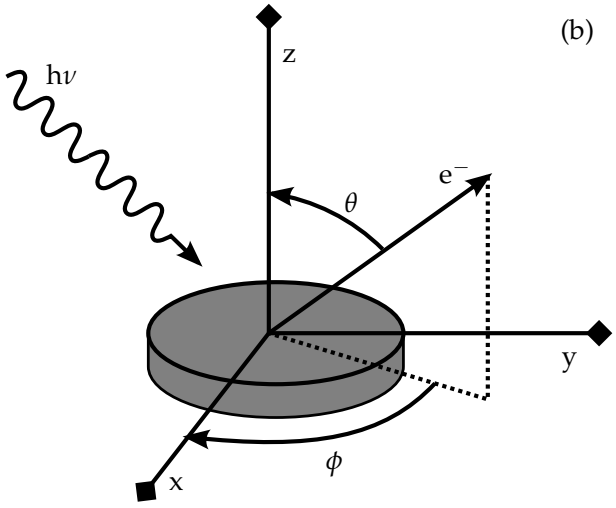

(b)

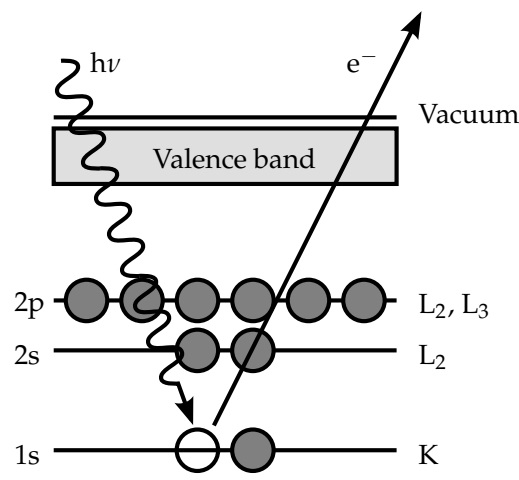

Figure 2.4: Schematic drawing of the XPS/XPD geometry (a). A sample mounted on a rotating sample stage is illuminated with $\mathrm{X}$-rays and the dependence of the photoelectron yield is recorded as a function of kinetic energy, azimuthal angle $(\phi)$ and polar angle $(\theta)$. The angle between the $X$-ray source and analyzer is fixed at $81^{\circ}$. Schematic drawing of the main XPS mechanism in (b): An energetic photon excites an atom, which emits an electron, leaving behind a core hole. The kinetic energy of such primary electrons can be accurately determined. A spectrum can be recorded that is a measure of the electronic structure of the sample.

\subsubsection{X-ray photoelectron diffraction}

XPD refers to performing angle dependent measurements of the photoelectron intensity, where the intensity modulations are caused by coherent scattering. Such scattering is typically observed in samples which show a high degree of order at the surface, like for example crystalline surfaces. XPD is highly surface sensitive, due to the limited electron mean free path in solids.

The diffraction or scattering patterns can be calculated using multiple scattering theory. ${ }^{[36]}$ Typically, a high intensity is observed along inter-atomic rows. Describing XPD patterns by analysis of atomic rows is called forward scattering or forwards focusing analysis. Work by for example $\mathrm{Xu}$ and van Hove ${ }^{\text {[37] }}$, Egelhoff Jr. ${ }^{[38]}$ and Poon and Tong [39], give a detailed description of this mechanism. In short, the emitted photoelectron wave, undergoes an attractive interaction with the neighboring cores (scatterers). Enhanced intensity is observed along a ring of solid-angle in the direction of the scattering atoms, due the cylindrical symmetry around the emitter-scatterer axis. The amount of 'forward focusing' depends on the kinetic energy of the photoelectrons and the distance between the atoms. At larger kinetic energies, electrons travel closer to the atom cores and the forward focusing cone becomes narrower. Typically these forward focusing effects are observed for kinetic energies above $500 \mathrm{eV}$. Next to forward focusing peaks, also interference peaks can be observed. The interatomic distance between the emitter and scatterer atoms has an effect on interference peaks. These interference peaks do not have to overlap with the forward focusing peaks. In some cases these interference peaks can even give rise to volcano shaped peaks in forward 
(a)

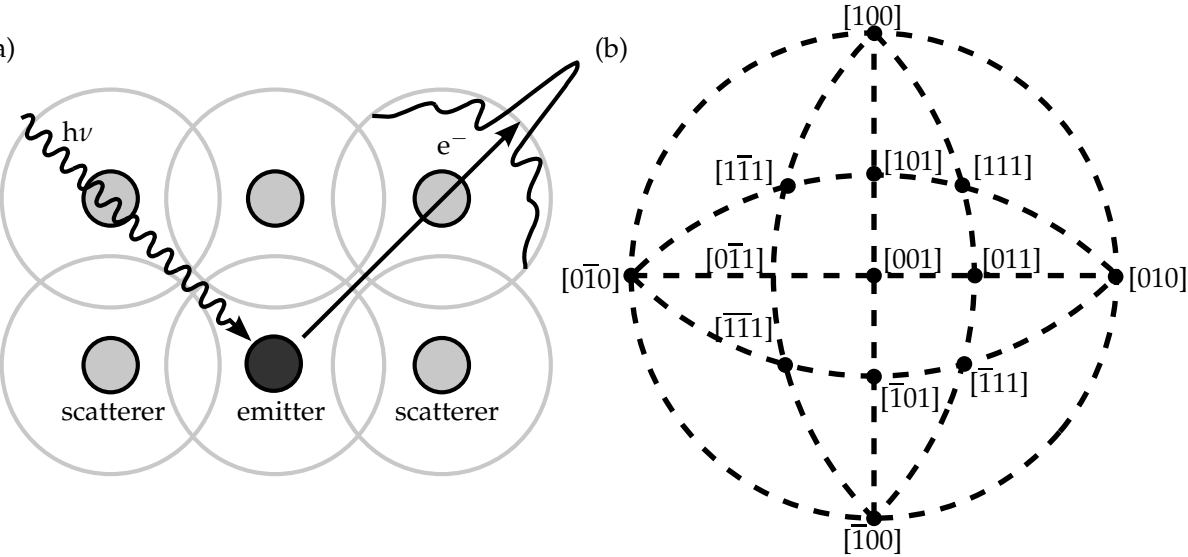

Figure 2.5: Schematic drawing of (a) multiple-scattering of spherical electron-waves resulting in a peaked angular distribution of electron intensity, originating from an emitter atom at a specific kinetic energy. Waves scattered of neighboring atoms (scatterers) gives rise to an interference pattern, with strong peaks along atomic-rows. In (b) a schematic stereographic projection of the emission pattern of a simple cubic (001) structure is drawn, some zone-axes are marked with their corresponding labels.

directions. A schematic of the XPD forward focusing and interference effect is shown in Fig. 2.5(a). A strong forward focusing peaks is drawn in the direction of a scatter, surrounded by interference type oscillations.

It is common to plot the scattering pattern obtained by XPD in stereographic projections. The locations of several crystal axis are shown in Fig. 2.5(b) using a stereographic projection. At the every intersection of two crystal planes, called the zone-axis, the directions are labeled for a simple cubic structure with the [001] direction pointing out-of-plane. The dotted lines represent families of crystal planes which are symmetric with respect to the labeled zone-axes.

\section{XPD Angular background}

The intensity in XPS depends on several factors, given in Eq. (2.3). ${ }^{\text {[40] }}$

$$
I=n f \sigma y \lambda A T
$$

Where, $n$ is the number of atoms per $\mathrm{cm}^{3}$ of the sample, $f$ is the X-ray flux, $\sigma$ is the photoelectric cross-section of the orbital which is probed, $\theta$ is the angular efficiency factor of the setup, $y$ is the efficiency of the photoelectric process, $\lambda$ is the electron mean free path in the sample, $A$ is the area of the sample which is probed by the analyzer and $T$ is the detector efficiency. By changing the rotation and tilt angle of the sample while the $X$-ray source and detector are fixed, several factors influence the angular dependence. By assuming a homogeneous sample, a constant incident flux and by probing a specific transition only two factors re- 
main: the angular efficiency of the setup and the area of the sample that is probed by the analyzer.

For an Omicron XPS system these functions are reported and explained in detail by Herrera-Gomez et al. ${ }^{[41]}$. The intensity is found to increase when the detector is facing parallel to the surface normal compared to facing parallel to the surface. Background functions similar to the ones shown by Herrera-Gomez et al. ${ }^{[41]}$ are shown in Fig. 2.6.

\section{XPD data acquisitions}

XPD data was recorded by measuring a narrow energy band near certain atomic transitions. Typically, spectra with about 35 data points and $20 \mathrm{eV}$ in width were recorded for every core-level of interest for every combination of angles. The measured core levels were selected based on two factors: the intensity should be high and the core-level should not overlap with core-levels of other elements. For example, $\mathrm{Sr} 3 \mathrm{~d}, \mathrm{O} 1 \mathrm{~s}$ and $\mathrm{Ti} 2 \mathrm{p}$ core levels were often recorded in the case of $\mathrm{SrTiO}_{3}$. Moreover, a background level was measured using a binding energy where no XPS peak was present.

A linear background was used to remove the XPS background from the spectral data. The XPD signal was then calculated by integrating the spectra. The angle-dependent spectral intensity of $\mathrm{O} 1$ s emission of an $\mathrm{Nb}$ doped $\mathrm{SrTiO}_{3}$ substrate is shown in Fig. 2.6. The raw data shows a strong angular $(\theta)$ dependence related to the instrument geometry, as discussed above. In order to extract the photoelectron diffraction contribution this background was removed. The following three methods for removing the angular background are discussed:

A Subtracting the recorded background data, at for example $1165 \mathrm{eV}$, from the core level data. The background data is first scaled to match the intensity of the selected core-level.

B Subtracting a $2^{\text {nd }}$-order polynomial fit from the core-level angular data.

C Normalizing all angular data at one specific energy value, i.e., the recorded background level at $1165 \mathrm{eV}$ binding energy

Results of all three background removal methods are shown in Fig. 2.6. In Fig. 2.6(a), method [A] is used. The background level recorded at $1165 \mathrm{eV}$ is scaled to average the intensity of the $\mathrm{O} 1$ s data and subsequently subtracted. The scaled background function is rather flat and does not appear to contain scattering effects. In Fig. 2.6(b), method [B] is applied. A polynomial background is subtracted from the recorded data. This approximation of the real background function is only valid for $\theta$ angles between roughly 20 and $70^{\circ}$. The results of method [C] are shown in Fig. 2.6(c). The spectral data is normalized at $1165 \mathrm{eV}$ by dividing by the background level. This introduces additional noise, because the intensity at the $1165 \mathrm{eV}$ is lower compared to the $\mathrm{O} 1$ s core level and similar measurement times were used. The insets show the effect of the selected background removal method on the stereographic projection of the intensity map. 


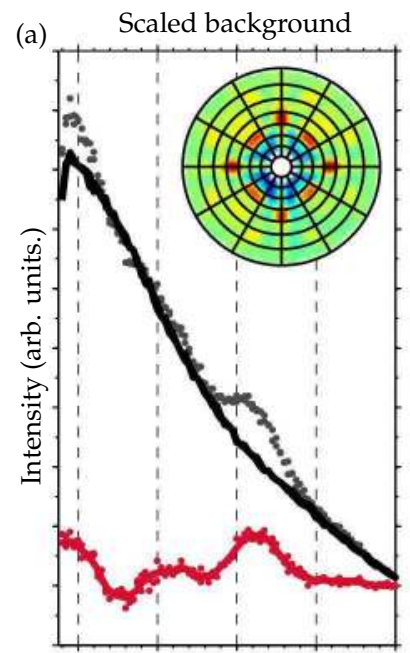

(b)

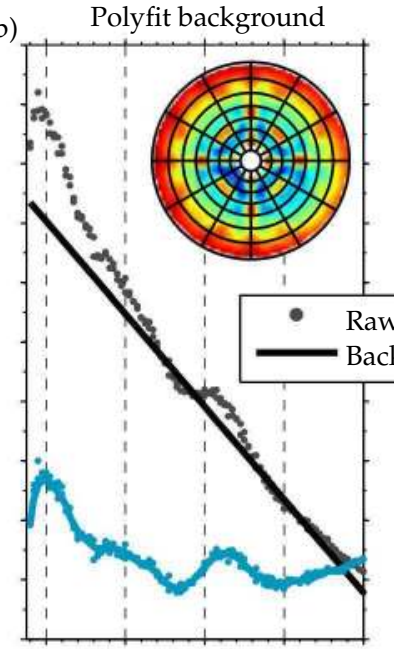

(c)

c) Normalized background

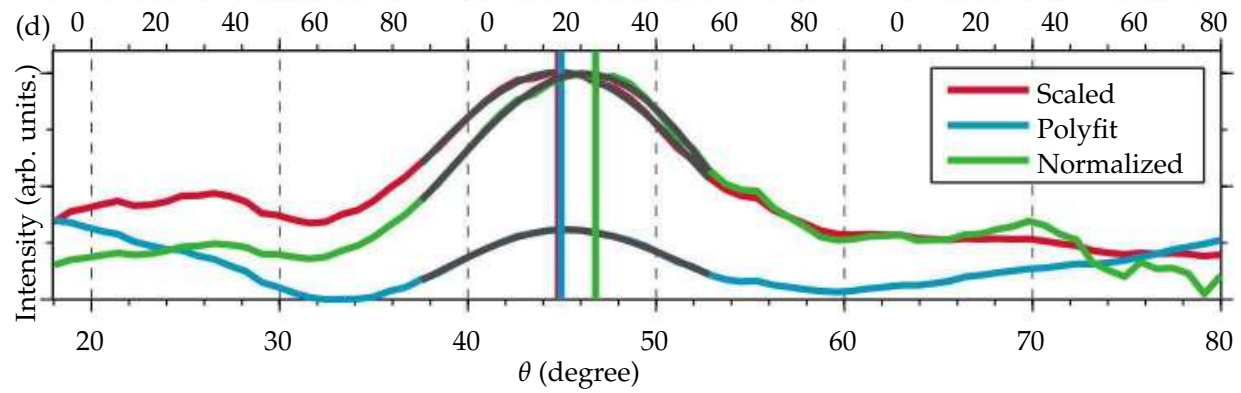

Figure 2.6: Results of three types of polar background removal methods for XPD for O 1s data of $\mathrm{SrTiO}_{3}$ (001). (a) Raw data, background recorded at $1165 \mathrm{eV}$ scaled to the data and data with the background function subtracted. (b) Raw data, $2^{\text {nd }}$ order polynomial background and data minus the background. (c) Raw data, background, background recorded at $1165 \mathrm{eV}$ and the result of normalizing the data to the background level. The insets in (a-c) show the resulting stereographic intensity map corresponding with the background function. In (d) $\theta$-scans with all three background removal methods are compared. The normalized background (c) gave a different main peak position compared both background subtraction methods. A three-point running average was used to smoothen the data.

In Fig. 2.6(d) the quantitative results of all three methods are compared. The main peak position near $45^{\circ}$ is determined using a Gaussian fit. For both methods [A] and [B] the peak is found at $\sim 45^{\circ}$. However, for method [C], the peak is found at $\sim 46.5^{\circ}$ which does not agree with simulations and forward focusing arguments. Therefore, in this work, methods [A] and [B] were used for removing the angle dependent background in XPD. 


\section{Angle resolved mass spectroscopy of recoiled ions}

Angle resolved mass spectroscopy of recoiled ions (AR-MSRI) ${ }^{7}$ was done using an Ionwerks time-of-flight mass spectrometer inside a high vacuum chamber with a background pressure below $10^{-6}$ mbar. This time-of-flight based mass spectroscopy technique was used for surface composition analysis. The intensity of recoiled ions strongly depends on surrounding ions as a result of blocking and/or shadowing effects. Therefore, azimuthal scans of the MSRI are sensitive to the in-plane structure of the sample. Prior to the measurements the samples were cleaned using trichloroethene, acetone and isopropanol in ultrasonic baths and the samples were heated to $500-600{ }^{\circ} \mathrm{C}$ in $0.07-0.13$ mbar oxygen to remove hydrocarbons. Potassium ions ${ }^{39} \mathrm{~K}$ were accelerated to $10 \mathrm{keV}$ and the incoming angle was set to $15^{\circ}$, while the azimuthal angle was scanned. Ion collection of masses up to $200 \mathrm{amu}$ was done in shadowing mode $\left(60^{\circ}\right)$ at $150{ }^{\circ} \mathrm{C}$.

\subsubsection{Magnetic and electrical characterization}

Next to X-ray photoemission analysis discussed above several other techniques were used to study the thin film samples prepared for this thesis. Characterization was focused towards studying the magnetic properties of $\mathrm{SrRuO}_{3}$.

\section{Polar magnetic-optic Kerr effect}

Polar magneto-optic Kerr effect (MOKE) experiments were performed using a loopless Sagnac interferometer at UCLA Irvine, California (USA) ${ }^{8}$. This instrument is capable of locally probing magnetism with spatial resolution as high as 1 micron. Magnetic Kerr sensitivity up to 10 nrad (less than $1 / 1000$ of a $\mathrm{ML}$ of $\mathrm{SrRuO}_{3}$ ) has been demonstrated down to temperatures as low as $0.5 \mathrm{~K}^{[42-44]}$. $\mathrm{T}_{C}$ was acquired from the Kerr versus temperature data by finding the maximum value of the second derivative.

\section{X-ray magnetic dichroism}

X-ray magnetic linear and circular dichroism (XMLD/XMCD) ${ }^{9}$ experiments were done at beamline ID08, at ESRF in Grenoble, France. The x-ray absorption spectra (XAS) were measured simultaneously by total electron yield and fluorescence yield detection. The photon beam was normal to the sample surface, i.e., parallel to the c-axis and to the external magnetic field. The XMCD signal was obtained by the difference of XAS measured with the left and right circularly polarized light $\mu^{+}$and $\mu^{-}$respectively, divided by the maximum at the L3-edge of the sum spectrum.

\footnotetext{
${ }^{7}$ AR-MSRI experiments performed at UC Berkeley, California USA (2011)

${ }^{8}$ MOKE experiments done in collaboration with Jing Xia and Sean Thomas, UCLA Irvine (20122013)

${ }^{9}$ X-ray dichroism experiments were done in collaboration with Gabriella Maria de Luca, SPIN CNR, Napoli, Italy (2011)
} 


\subsection{Simulations and computer modeling}

Several types of computer simulations and calculations were used to explain and model the observed experimental phenomena. Multiple scattering (MS) simulations were used to calculate scattering patterns of samples analyzed by XPD, see chapter 6. Density functional theory was used to model the effect of strain on the octahedral tilt patterns and magnetic properties of $\mathrm{SrRuO}_{3}$ in chapter 7. Kinetic Monte Carlo growth simulations were performed to model to self-organization of $\mathrm{SrRuO}_{3}$ in chapter 4 .

\subsubsection{Multiple scattering simulations}

Multiple scattering electron diffraction simulations were done using EDAC ${ }^{[36]}$ software, with a cluster size of $\sim 700$ atoms, a mean free path of $2.3 \mathrm{~nm}$ and using 10 iteration steps.

\subsubsection{Density functional theory calculations}

Density functional theory $(\mathrm{DFT})^{10}$ calculations were performed using VASP code. The spin-polarized generalized-gradient approximation GGA (PBE) was used for calculating the effects of the capping layer. LDA+U and GGA were used to for studying the bare $\mathrm{SrRuO}_{3}$ films. For the simulations, the energy cut-off was set to $500 \mathrm{eV}$, and a $12 \times 12 \times 8 \mathrm{k}$-point mesh was used to sample the Brillouin zone. The lattice constant of $\mathrm{SrRuO}_{3}$ were first optimized, yielding $\mathrm{a}=5.593 \AA$, b $=5.606 \AA$, and $\mathrm{c}=7.884 \AA$.

\subsubsection{Monte Carlo growth simulations}

Atomistic simulations of PLD growth were done using Monte Carlo type models. In such simulations atomic movement of adatoms ${ }^{11}$ on a surface is performed based on activated processes, i.e., deposition, evaporation and diffusion. A schematic of these processes is given in Fig. 2.7(a), where also the nucleation of two adatoms is drawn. The simulations were performed on a lattice or grid with a cubic structure. Periodic boundary conditions were used to simulate adatoms moving across the borders of the grid. The pulsed nature of PLD was simulated by simultaneous deposition of a bunch of adatoms on random grid sites and no evaporation is taken into account. The diffusion hopping rates $k_{i}$ for event $i$ is calculated using Eq. (2.4).

$$
k_{i}=k_{0} \cdot e^{\left(\frac{-E_{\mathrm{D}}}{k_{\mathrm{B}}}\right)}
$$

\footnotetext{
${ }^{10}$ DFT calculations done by Zhicheng Zhong, University of Twente (2011) and TU Wien, Austria (2012)

${ }^{11}$ In these simulations the PLD growth process is simplified; only deposition and diffusion singular entities is allowed. Typically, these entities are atoms (adatoms) when simulating growth of for example metals. However, in the case of perovskites, diffusion and deposition of complete unit-cell blocks are simulated, which are referred to as adatoms here.
} 
(a)

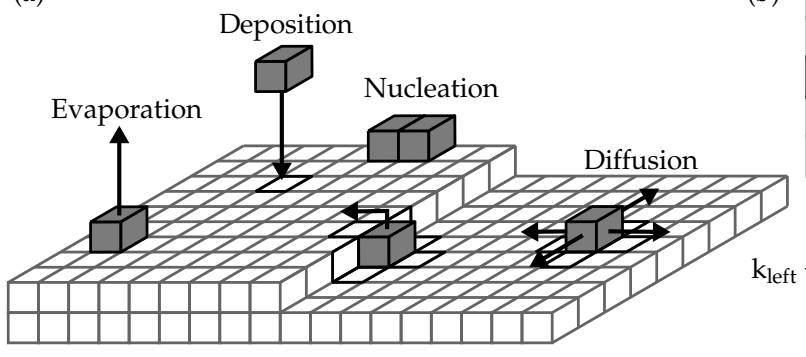

(b)

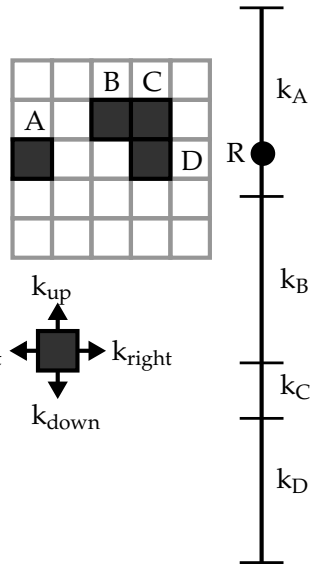

Figure 2.7: Overview (a) of the simulated processes, evaporation, deposition, diffusion and nucleation. (b) Schematic of the event selection procedure for four adatoms A, B, $\mathrm{C}$ and $\mathrm{D}$. Their hopping rates are depicted on the right, the highest rate found for $\mathrm{A}$ is indicated by the longest line segment. A random number $R \leq \sum k_{i}$ is selected and its position on the event list determines which event is selected, in this case $k_{A}$.

Where, the event rate $\mathrm{k}_{i}$, for diffusion of a single adatom, depends on the hopping attempt frequency $\mathrm{k}_{0}$, the temperature $\mathrm{T}$, the Boltzmann constant $\mathrm{k}_{\mathrm{B}}$ and the activation energy for diffusion, $E_{D}$. The definition of the activation energy is of the form: $\mathrm{E}_{\mathrm{D}}=\mathrm{E}_{\mathrm{S}}+\mathrm{n} \cdot \mathrm{E}_{\mathrm{N}}$, where $\mathrm{E}_{\mathrm{S}}$ is a static contribution and $\mathrm{E}_{\mathrm{N}}$ scales with $n$, the number of nearest neighbors. This so-called lattice kinetic Monte Carlo (kMC) algorithm allows for accurate determination of the simulated time; the time interval of one simulation event is inversely proportional to the total hopping rate, $K$. This kMC type algorithm is described below.

\section{Simulation algorithm}

The event selection is based on random numbers which are acquired using a Mersenne Twister ${ }^{[45]}$ pseudo-random number generator. The code for the algorithm is written in C++, initially compiled using Microsoft Visual Studio 2010, but most simulations were performed using the GNU Compiler Collection on a linux platform. Selecting an event in an ordered list of possible events, $k_{i}$ is done by using a binary chop type algorithm. A random number $(r \cdot K)$ is generated and the corresponding event is searched for. A schematic of such a selection procedure is given in Fig. 2.7(b). The chop algorithm first determines if the selected random number is in the first half of the ordered list of events by calculating $\sum_{i=1}^{N / 2} k_{i}$ and continues doing the same in either the first or second half of the list until a single event is found. A fast Monte Carlo algorithm ${ }^{[46]}$ is also used where the list of events is split into groups. 


\section{KMC algorithm}

1 Set time $t=0$ and load the initial simulation parameters. For example define a terrace step substrate grid with dimensions $x$ and $y$.

2 Deposit $N_{a}$ atoms: $N_{a}=\frac{x \cdot y}{P p m l}$ where the growths speed, Ppml is the number deposition pulses required to complete one monolayer.

3 Calculate all neighbor counts $n$ and all hopping rates:

$k_{i}=k_{0} \cdot e^{\left(\frac{-E_{\mathrm{D}}}{k_{\mathrm{B}} T}\right)}$ where $\mathrm{E}_{\mathrm{D}}=\mathrm{E}_{\mathrm{S}}+\mathrm{n} \cdot \mathrm{E}_{\mathrm{N}}$.

4 Calculate the cumulative hopping rate: $K=\sum_{i=1}^{N} k_{i}$.

5 Select one event using a random number $r \in[0,1]$ and find the event with a hopping rate: $K_{i-1}<r \cdot K \leq K_{i}$

6 Perform the hopping event

7 Update all relevant hopping rates which might have changed.

8 Update the simulation time $t=t+\Delta t$, where $\Delta t=-\frac{\log (r)}{K}$.

9 Based on the simulation time, either deposit more material (return to step 2) or return to step 3 and perform another hopping event. 


\title{
Chapter 3
}

\section{$\mathrm{DyScO}_{3}(\mathbf{1 1 0 )}$ substrate surface termination control}

\begin{abstract}
The surface structure and termination of high quality single crystal $\mathrm{DyScO}_{3}$ (110) substrates is studied and controlled for their use as substrates for growth of oxide thin films and nanostructures. A selective wet chemical etching procedure, used for creating singly $\mathrm{ScO}_{2}$ terminated surfaces, is enhanced by the addition of a surface roughening step. The structure of the $\mathrm{DyScO}_{3}$ (110) surfaces is analyzed using atomic force microscopy, time-of-flight mass spectroscopy, reflection high energy electron diffraction and surface $\mathrm{X}$-ray diffraction in various stages of the surface treatment process. These well-defined singly terminated $\mathrm{DyScO}_{3}$ surfaces are used as a starting point in order to artificially induce a DyO terminated surface. Moreover, ordered mixed terminated $\mathrm{DyScO}_{3}$ templates are obtained which can serve as templates for nanowire growth, discussed in chapter 4 .
\end{abstract}

Part of the work discussed in this chapter is published in: J.E. Kleibeuker, B. Kuiper, S. Harkema, D.H.A. Blank, G. Koster, G. Rijnders, P. Tinnemans, E. Vlieg, P.B. Rossen, W. Siemons, G. Portale, J. Ravichandran, J.M. Szepieniec, and R. Ramesh. Physical Review B 85, 165413 (2012). ${ }^{[47]}$ 


\subsection{Introduction}

High quality and well-defined single crystal perovskite-type oxide substrates are of great interest for creating strained oxide films. By using different substrate materials which apply different amounts of epitaxial strain, it is possible to tune existing properties ${ }^{[22]}$, as well as reveal new properties. ${ }^{[12]}$ Moreover, due to the polar nature of some perovskite oxides, the exact interface between the substrate and film material in epitaxial structures can give rise to new and intriguing phenomena. ${ }^{[10]}$ To be able to fully control and tune such properties and interface configurations, the surface structure of the substrate should be well-defined and well-controlled.

One can consider the unit-cell of a perovskite-type oxide in a (pseudo-)cubic form as a stack of alternating planes of $\mathrm{AO}$ and $\mathrm{BO}_{2}$, as depicted in Fig. 3.1(a). After cleaving, both types of planes will be present at the surfaces of the cleaved crystal parts. The resulting surfaces contain equal amounts of both types of surface terminating layers. However, the spatial distribution of different types of surface termination varies locally, as sketched in Fig. 3.1(b). For atomically controlled growth of two-dimensional layered sheets, a single type of surface termination covering the surface is desired as a starting point, as drawn in Fig. 3.1(c).

Popular perovskite substrates for thin film growth are shown in Fig. 3.1(d). The substrates are ordered based on their lattice parameter, which is important when considering epitaxial strain in thin films. Next to, for example, $\mathrm{SrTiO}_{3}$, $\mathrm{LaAlO}_{3}$ and LSAT, the group of rare-earth scandates $\left(\mathrm{REScO}_{3}\right)$ is of high interest, due to its high crystalline quality and the slightly larger lattice parameters. The surface structure of $\mathrm{SrTiO}_{3}$ is studied in great detail, e.g., by studying the possible types of surface reconstructions. ${ }^{[48-50]}$ The surface termination of $\mathrm{SrTiO}_{3}$ can be well controlled by chemical etching to yield surface with a $\mathrm{TiO}_{2}$ termination. ${ }^{[25,51,52]}$ Surfaces with a dominant $\mathrm{SrO}$ termination can also be prepared. ${ }^{[53,54]}$ This controlled surface termination makes $\mathrm{SrTiO}_{3}$ one of the most popular substrates for thin film growth of perovskite-type oxides.

Rare-earth scandate crystals, like $\mathrm{DyScO}_{3}$, can be prepared using the Czochralski growth technique, resulting in high crystalline quality and strain free crystals. Compared to $\mathrm{SrTiO}_{3}$, made by Verneuil growth, the number of defects is significantly reduced. The $\mathrm{REScO}_{3}$ group is isostructural with $\mathrm{GdFeO}_{3}$ and has an orthorhombic (Pbnm, spacegroup \#62) crystal structure. $\mathrm{DyScO}_{3}$ has the following lattice parameters ${ }^{[18,20]}: \mathrm{a}=0.5440 \mathrm{~nm}, \mathrm{~b}=0.5717 \mathrm{~nm}, \mathrm{c}=0.7903 \mathrm{~nm}$, corresponding to a pseudo-cubic lattice parameter of $\mathrm{a}_{p} \sim 0.395 \mathrm{~nm}$. All rare-earth scandates are insulating with a bandgap above $5.5 \mathrm{eV} \cdot{ }^{[21]}$ Strong magnetic anisotropy is found in $\mathrm{DyScO}_{3}{ }^{[55]}$ and an anti-ferromagnetic phase is found at temperature below $3.1 \mathrm{~K}$, which should be considered when studying magnetism in films on $\mathrm{DyScO}_{3}$ substrates.

As mentioned above, surfaces with a single type of surface termination are desired for growth of complex oxide heterostructures in order to form two-dimensional layered sheets of various oxides with a vast range of physical proper- 

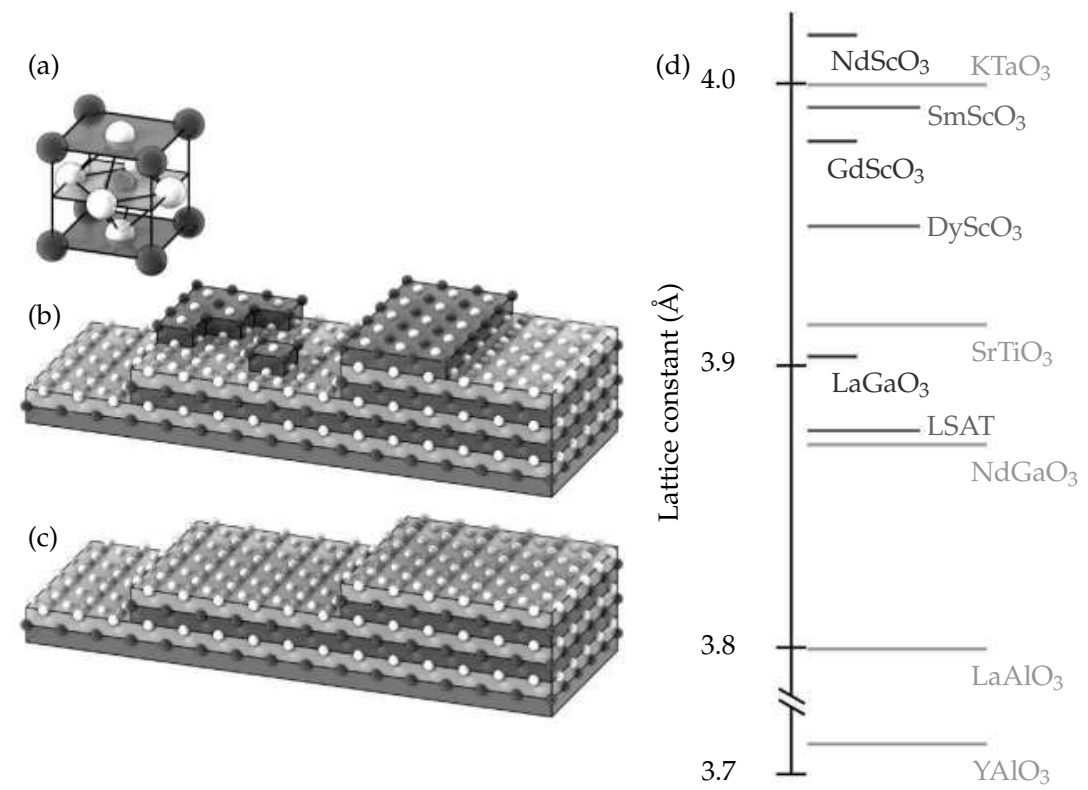

Figure 3.1: Schematic drawing of (a), a cubic $\mathrm{ABO}_{3}$ perovskite unit-cell, (b) a mixed terminated surface and (c) a surface with a single type of terminating layer, adapted from Kleibeuker et al. ${ }^{[26]}$. (d) Overview of commonly used perovskites, adapted from Schlom et al. ${ }^{[22]}$.

ties. However, an interesting case of ordered areas of both surface termination is also considered, as shown by Foerster et al. ${ }^{[56]}$, where a laterally confined twodimensional electron gas is created on $\mathrm{SrTiO}_{3}$ surface with ordered areas of $\mathrm{SrO}$ and $\mathrm{TiO}_{2}$ surface termination. Moreover, the growth of $\mathrm{SrRuO}_{3}$ is highly sensitive to the surface termination of oxide substrates. This sensitivity allows for $\mathrm{SrRuO}_{3}$ self-organization ${ }^{[57,58]}$ based on the termination template, resulting in conducting nanostructures on an insulating substrate. However, reproducible acquisition of mixed ordered termination patterns is not shown in the literature.

The $\mathrm{DyScO}_{3}$ (110) surface morphology and structure are studied on $\mathrm{DyScO}_{3}$ surfaces with various treatments, e.g., annealed and chemically etched. In this chapter, the number of treatment steps applied to the $\mathrm{DyScO}_{3}$ substrate is increased in every subsequent section. First, the surface morphology of annealed $\mathrm{DyScO}_{3}$ substrates is studied using atomic force microscopy (AFM). The observed height distributions are categorized in distinct groups. Ordered mixed terminated substrates are selected based on the AFM results, which can be used as templates for the growth of $\mathrm{SrRuO}_{3}$ nanowires, as discussed in chapter 4 . Next, a chemical treatment is introduced, in order to achieve single $\mathrm{ScO}_{2}$ termination. The exact surface structure such $\mathrm{ScO}_{2}$ terminated surfaces and other $\mathrm{DyScO}_{3}$ surfaces are studied using various surface sensitive techniques, like surface $X$ ray diffraction (SXRD), reflection high energy electron diffraction (RHEED) and 

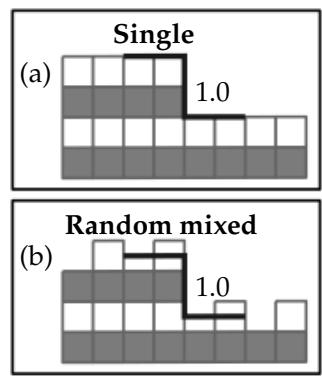
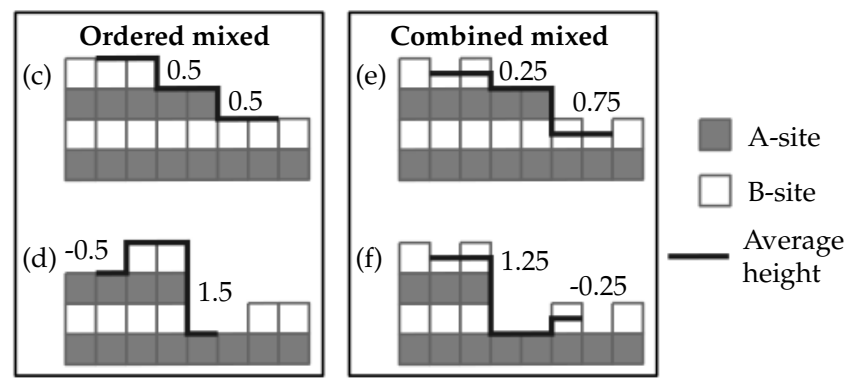

Figure 3.2: Surface termination models for layered perovskites. The sketch in (a) represents a singly terminated surface, (b) a randomly mixed terminated surface, $(c, d)$ two types of ordered mixed terminated surfaces and $(\mathrm{e}, \mathrm{f})$ two combinations of random and ordered mixed terminated surfaces. The dark line profiles indicate average terrace height profiles for the given morphologies. The corresponding step size are also given in the figure, where 1.0 is the size of one pseudo-cubic unit-cell $(\sim 4 \AA)$.

time-of-flight mass spectroscopy (TOF-MS). The combined studies using these techniques and $\mathrm{SrRuO}_{3}$ growth by pulsed laser deposition (PLD) give a detailed description of the chemically treated $\mathrm{DyScO}_{3}(110)$ surface and provide insight in other possible surface configurations. Finally, the PLD growth of an DyO overlayer is discussed to create $\mathrm{DyScO}_{3}$ surfaces with an artificially induced DyO surface termination.

\subsection{Mixed surface structure of annealed $\mathrm{DyScO}_{3}$}

Cleaved and polished perovskite surfaces (as-received) have a disordered surface morphology, which is often hard to quantify. Therefore, an anneal step is performed, to create an ordered starting point for thin film growth, which can be readily analyzed using AFM. During annealing the surfaces reorganizes, forming terrace structures with a step width related to the miscut angle between the crystal axis and the actual surface. After cleaving and polishing ${ }^{1}$ the resulting surfaces must on-average contain an equal amount of both $\mathrm{A}$ and $\mathrm{B}$ cations at the surface.

During high temperature annealing several processes influence the resulting surface morphology; (1) Surface reorganization via diffusion; (2) Cation diffusion through the bulk to the surface, mostly near crystal defects; (3) Evaporation of material. Since $\mathrm{DyScO}_{3}$ has a melting point well above the anneal temperature and the crystal quality of $\mathrm{DyScO}_{3}$ is very high, the first mechanism is assumed dominant. The second and third process become more pronounced at high temperature or using long anneal times.

The distribution of $\mathrm{A}$ and $\mathrm{B}$ cation terminated surface areas after annealing can have different degrees of order. A schematic of some possible surface config-

\footnotetext{
${ }^{1}$ The polishing is assumed to be non-preferential with respect to the surface termination.
} 
urations is shown in Fig. 3.2. For (a), a singly terminated surface, (b) a configuration where the termination is random and $(c, d)$ surfaces with ordered areas of both cation species at the surface. Combinations of ordered and randomly terminated surface areas are depicted in $(\mathrm{e}, \mathrm{f})$. In schematics the random mixed and ordered mixed diagrams have a termination ratio $(\mathrm{A} / \mathrm{B})$ of 0.5 , the single of 1.0 and the combined of 0.25 . Two more mixed configurations can be made where the location of the A or B terminated areas is changed with respect to the terrace step by interchanging A and B in the patterns in (c) and (d). Similar additional configurations for the combined termination configuration are also possible as well as different termination ratios.

The dark line drawn at the surface of all configurations indicates the average height profile. In the case of single and ordered mixed termination, the height profile is drawn equal to the local sample surface. However, an AFM tip might not be able to completely resolve the mixed termination in the case of a randomly terminated surface. Depending on the lateral size of the mixed termination features, and AFM might fully resolve them latterly, observe only the highest features or measure an averaged height value. The latter is assumed in the sketch and will be shown to be feasible in the experimental results discussed below. The resulting possible height steps observed on (averaged) height profiles show steps of 1 and 0.5 unit-cell (u.c.) for the ordered single and ordered mixed terminated surfaces. For the random mixed terminated areas of the surface, averaging of the height profile can result in observed step height of 0.25 u.c. and 1.25 u.c.

It is interesting to note that complete single termination of a bulk $\mathrm{DyScO}_{3}$ crystal will lead to a polar interface, due to the charged nature of the alternating $\mathrm{DyO}^{+}$and $\mathrm{ScO}_{2}^{-}$planes. This polar surface-vacuum interface must be resolved at the surface, possibly by vacancies, reconstructions or adsorbates. For highly ordered mixed terminated surfaces this polar surface must also be resolved at a local scale.

In this section, the morphology of annealed $\mathrm{DyScO}_{3}$ surfaces is studied using AFM. Height as well as phase and friction force imaging provide insight into the termination ordering. The observed morphologies after annealing are related to the models described above.

\subsection{1 $\mathrm{DyScO}_{3}$ annealing for mixed termination}

Various annealed $\mathrm{DyScO}_{3}$ substrates were studied using AFM, as described in section 2.2.1. The resulting micrographs are depicted in Fig. 3.3. In all height images, clear terrace structures were observed, with miscut angles between $0.1^{\circ}$ and $0.2^{\circ}$ for all samples, except sample (a), which had a lower miscut angle of $\sim 0.05^{\circ}$.

In Fig. 3.3(a) a clear phase contrast is visible. While, in the corresponding height image and line profile, almost no contrast is visible. A height line profile for an ordered mixed terminated surface with a step structure of $0.6 \mathrm{~nm}$ up and $0.2 \mathrm{~nm}$ down is drawn on top of the line profile data in (a). However, the average height profile showed steps of $0.5 \mathrm{~nm}$ up and $0.1 \mathrm{~nm}$ down. This indicates a 

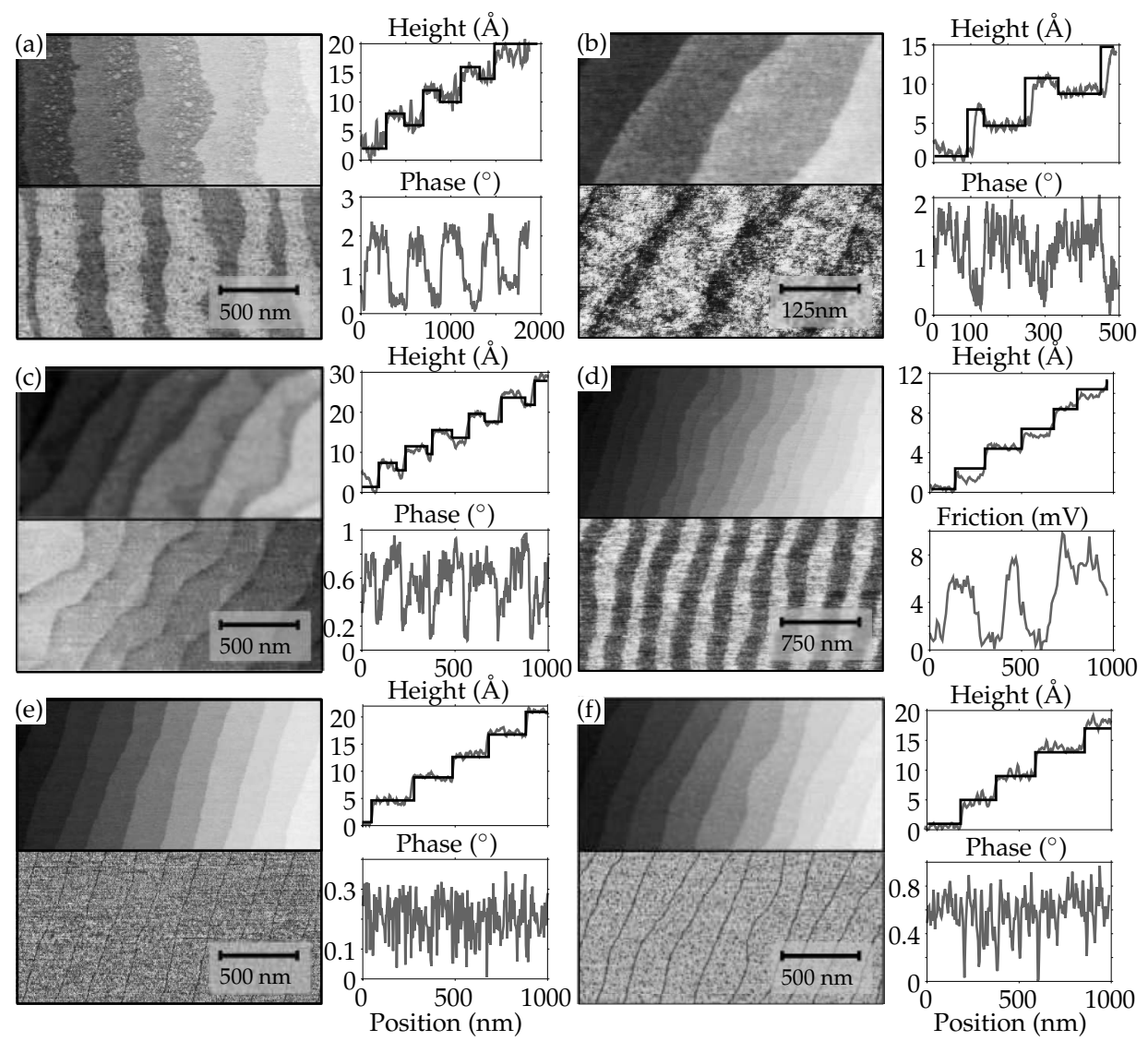

Figure 3.3: Examples of mixed terminated $\mathrm{DyScO}_{3}$ substrates after annealing in oxygen. $(\mathrm{a}, \mathrm{b}, \mathrm{c}, \mathrm{e}, \mathrm{f})$ simultaneously recorded TM-height and TM-phase micrographs of five different substrates and corresponding line profiles. (d) TM-height and CM-friction images one substrate, recorded sequentially at different positions. Black lines in the line profiles provide a guide to the eye. For (a-c) steps of 0.2 and $0.6 \mathrm{~nm}$ are drawn, for (d) steps of $0.2 \mathrm{~nm}$ only are drawn and for $(\mathrm{e}, \mathrm{f})$ steps of $0.4 \mathrm{~nm}$.

surface configuration as drawn in Fig. 3.2(f).

The surface areas corresponding to the dark phase contrast in Fig. 3.3(a) had a standard deviation of the height of $0.06 \mathrm{~nm}$. Whereas, the areas corresponding to the white phase contrast had a standard deviation of $0.12 \mathrm{~nm}$. This variation in roughness could also be observed qualitatively in the height image. This change is roughness is in agreement with the observed averaged height steps of 0.25 and 1.25 u.c. This samples shows that the AFM might not be able to fully resolve areas of mixed termination latterly and thus provide an averaged height value on terrace areas with a higher roughness, as discussed above.

In Fig. 3.3(b) a clear phase contrast is visible. However, almost no height con- 
trast was observed, similar to (a). A mixed termination configuration similar to (a) is drawn on top of the data. Steps of $0.5 \mathrm{~nm}$ up and $0.1 \mathrm{~nm}$ down were observed using averaged height profiles, but no clear roughness changes were observed, indicating a surface model as plotted in Fig. 3.2(d) or (f).

Sample (c) ${ }^{2}$ in Fig. 3.3 showed a clear height contrast with two distinct height levels on each substrate terrace. However, no clear phase contrast was observed. The step structure indicated an ordered mixed terminated substrate surface, as drawn schematically in Fig. 3.2(d).

Figure $3.3(\mathrm{~d})^{3}$ shows height and friction data. A clear friction contrast was observed, as well as two distinct height levels on each terrace. The height profile indicates surface steps of $0.3 \mathrm{~nm}$ and $0.1 \mathrm{~nm}$ steps upwards, similar to the configuration in Fig. 3.2(e).

Samples shown in Fig. 3.3(e) and (f) showed clear terrace steps. The line profiles indicates only $0.4 \mathrm{~nm}$ steps upwards, while no ordered phase contrast was observed. The surface roughness of (f) was slightly higher compared to (e), because (f) had small islands or dots on the terraces, also giving rise to some spots in the phase image. The step structure of $0.4 \mathrm{~nm}$ could match with a singly terminated surface (Fig. 3.2(a)), as well as a randomly mixed terminated surface (Fig. 3.2(b)).

Note, these samples are merely a selection of $\mathrm{DyScO}_{3}$ surface morphologies obtained after annealing. Surface morphologies resembling Fig. 3.3(e,f) were observed most frequently after annealing of $\mathrm{DyScO}_{3}$ substrates with a similar miscut. Based on the combined analysis of height and phase/friction images it possible to estimate the surface termination configuration. However, for many samples it is not possible to do this unambiguously, especially when the samples appear to be singly terminated. Therefore, the use of AFM alone is not sufficient to determine the surface termination configuration. Moreover, AFM does not allow for determining the local type of surface termination; AFM can only show a contrast, with respect to areas with a different surface termination.

\subsubsection{Influence of the anneal parameters}

As discussed above, various surface configurations were observed after annealing of $\mathrm{DyScO}_{3}$ substrates under similar conditions. No clear dependence of the miscut angle on the configuration was observed. Ordered mixed termination was observed on substrates annealed for 30 minutes to 12 hours (not shown). Whether or not ordered mixed termination was observed could not be predicted based on miscut or by selecting the anneal time.

In order to study the time evolution of the surface termination configuration during annealing a single $10 \times 10 \mathrm{~mm}^{2} \mathrm{DyScO}_{3}$ substrate was divided into pieces, which were annealed separately for various times. AFM height and friction images of pieces annealed for 30 minutes, 4 hours and 8 hours are depicted in Fig. 3.4. After 30 minutes the steps and terraces were ordered and showed

\footnotetext{
${ }^{2}$ AFM data recorded by David Dubbink (2010, University of Twente)

${ }^{3}$ AFM data recorded by Brian Smith (2012, University of Twente)
} 

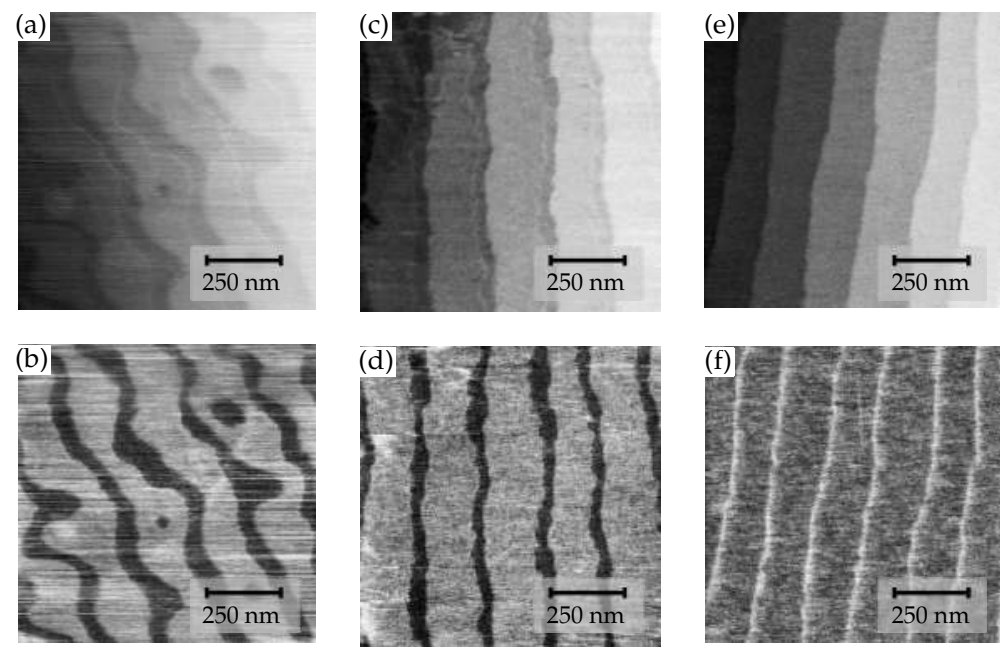

Figure 3.4: $\mathrm{DyScO}_{3}$ annealed for 30 minutes $(\mathrm{a}, \mathrm{b}), 4$ hours $(\mathrm{c}, \mathrm{d})$ and 8 hours $(\mathrm{e}, \mathrm{f})$ AFM height $(\mathrm{a}, \mathrm{c}, \mathrm{e})$ and friction force $(\mathrm{b}, \mathrm{d}, \mathrm{f})$ images.

two distinct levels, both in height as well as in friction force contrast. Annealing longer, 4 hours, resulted in straightening of the step edges and a higher degree of order. The ratio of mixed termination was changed. The amount of terrace area with a lower height was reduced upon annealing. The same trend was observed when annealing for 8 hours. Where only steps of $0.4 \mathrm{~nm}$ were observed and no friction force contrast was found, expect for some signal at the step edge, which was an experimental artifact caused by the step edge.

\subsection{Surface structure of $\mathrm{DyScO}_{3}(110)$}

Annealing of $\mathrm{DyScO}_{3}$ yields surfaces with various degrees of order. Substrates with a high degree of termination ordering can be used to create nanostructures, as discussed in chapter 4 . However, well-defined oxide surfaces with a single type of surface termination are of great interest for the study and development of new and enhanced (interface) properties of perovskite type materials. The surface structure of $\mathrm{DyScO}_{3}$ and the surface treatment are hardly addressed in literature. Therefore, below a selective chemical etching procedure is discussed to create singly $\mathrm{ScO}_{2}$ terminated substrates. $\mathrm{DyScO}_{3}(110)$ is used as a model system for the scandate group of perovskites. The resulting $\mathrm{ScO}_{2}$ terminated surfaces are studied using RHEED, TOF-MS and SXRD in order to determine the surface structure. 


\subsubsection{Selective wet chemical etching for $\mathrm{ScO}_{2}$ termination}

A selective wet chemical etching procedure for $\mathrm{DyScO}_{3}$ is introduced and discussed in detail by Kleibeuker et al. ${ }^{[26]}$. The process consists of two main steps; (1) forming a hydroxide and (2) dissolving this hydroxide. A similar method is applied successfully to $\mathrm{SrTiO}_{3}$ (001) surfaces. Immersing $\mathrm{SrTiO}_{3}$ in water results in the formation of $\mathrm{Sr}(\mathrm{OH})_{2}$, which is readily dissolved in an $\mathrm{HF}_{-}-\mathrm{HNO}_{3}$ solution ${ }^{[25,51]}$. Recently, $\mathrm{Sr}(\mathrm{OH})_{2}$ is also reported to dissolve in demineralized water during ultra-sonic agitation. ${ }^{\text {[52,59] }}$

In the case of $\mathrm{DyScO}_{3}$, the reaction in Eq. (3.1) is expected to dominate the etching process in basic solutions.

$$
\mathrm{Dy}_{2} \mathrm{O}_{3}(\mathrm{~s})+3 \mathrm{H}_{2} \mathrm{O}(\mathrm{l}) \stackrel{6 \mathrm{OH}^{-}(a q)}{\longrightarrow} 2 \mathrm{Dy}(\mathrm{OH})_{3}(a q)
$$

Here $\mathrm{OH}^{-}$acts as a catalyst. Both the hydroxide formation and the dissolving of the hydroxide can be combined into one step for $\mathrm{DyScO}_{3}$ by using a $12 \mathrm{M}$ $\mathrm{NaOH}$, water solution. Kleibeuker et al. ${ }^{[26]}$ have shown this method to be successful for creating singly terminated $\mathrm{DyScO}_{3}$ surfaces.

\section{Enhancement of etching rate}

In order to confirm single termination, $\mathrm{SrRuO}_{3}$ thin films were grown on the treated $\mathrm{DyScO}_{3}$ substrates. $\mathrm{SrRuO}_{3}$ is highly sensitive to the local surface termination, as it preferentially nucleates on B-site terminated surface areas. ${ }^{[57,58,60]}$ Therefore, this sensitivity ${ }^{4}$ was used to confirm the perfect single termination of treated $\mathrm{DyScO}_{3}$ substrates. $\mathrm{SrRuO}_{3}$ growth experiments indicated that the chemical treatment did not always result in perfect $100 \%$ single termination, as will be discussed below.

The surface morphologies obtained after the individual treatment steps of two $\mathrm{DyScO}_{3}$ (110) substrates and subsequent $\mathrm{SrRuO}_{3}$ growth results are shown in Fig. 3.5. A substrate annealed for 4 hours (a), showed atomically smooth steps without indication of ordered mixed surface termination. The surface was still atomically smooth, after selective etching in $12 \mathrm{M} \mathrm{NaOH}(\mathrm{b})$. Interestingly, the small protrusions (spots) on the terraces observed after annealing were not observed after etching. These spots were possibly caused by contaminations or small islands of $\mathrm{DyO}$ or $\mathrm{DyScO}_{3}$. Growth of $\mathrm{SrRuO}_{3}$ with expected film thickness of 5 mono-layers resulted in the undesired formation of islands with a height of up to $4 \mathrm{~nm}$. This island formation indicates the $\mathrm{DyScO}_{3}$ substrate surface was not singly terminated.

To increase the selective etching rate, a surface roughening step was introduced. Such a surface roughening step could remove the dependence of the etching result on the substrate surface morphology prior to wet chemical etching. As $\mathrm{DyScO}_{3}$ was annealed prior to chemical etching, the surface was smoothened and the number of step edges was different for every sample, as is clear from the

\footnotetext{
${ }^{4}$ This sensitivity is used in chapter 4 to create $\mathrm{SrRuO}_{3}$ nanostructures.
} 

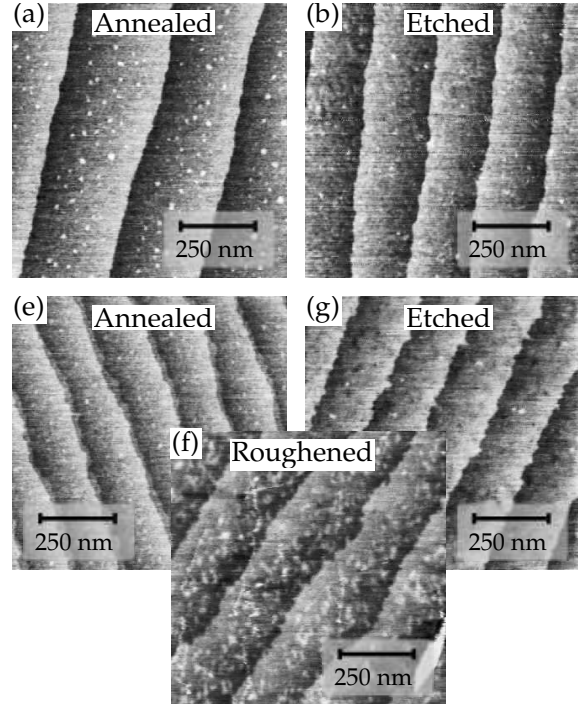

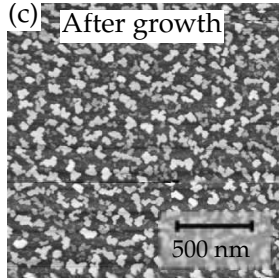

(h) After growth

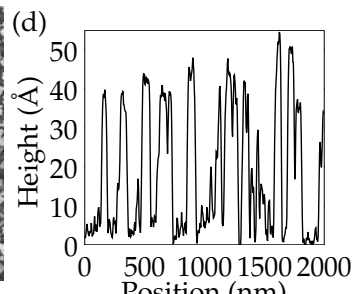

Position (nm)

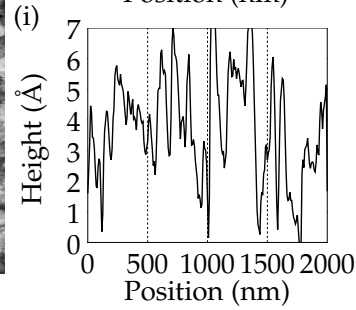

Figure 3.5: Example of surface preparation process for two $\mathrm{DyScO}_{3}$ (110) substrates. In (a) and (b) the surface morphology of a substrate is shown after annealing and after subsequent $\mathrm{NaOH}$ etching. The resulting growth of $\sim 5$ unit-cell layers of $\mathrm{SrRuO}_{3}$ resulted in islands with a height of $\sim 4 \mathrm{~nm}$ as indicated in (c) and (d). After annealing of a second substrate (e), a surface roughening step (f) was performed by using a strong acid, resulting in a higher surface roughness. After subsequent $\mathrm{NaOH}$ etching $(\mathrm{g})$, a smooth surface was obtained again. $\mathrm{SrRuO}_{3}$ growth resulted in a smooth film surface after deposition of $\sim 12$ unit-cell layers, as indicated in (h) and (i).

range of different morphologies observed in Fig. 3.3. Moreover, the distribution of A and B-site terminated surface areas showed different degrees of order. At the step edges, Dy has a lower coordination number than on the terraces. Therefore, the etching rate at step edges or at the boundary of A/B-site terminated areas is expected to be enhanced compared to atomically flat terrace areas. A similar asymmetry in etching rate is observed for $\mathrm{Si}$ (111) surfaces, where the lowest etching rate is observed on H-terminated (111) planes. ${ }^{\text {[61] }}$

The surface roughening step was introduced in between the annealing and selective etching process. The $\mathrm{DyScO}_{3}$ substrate was dipped in buffered HF, for 30-60 seconds in an ultrasonic bath. The AFM height images of the surface at several stages of this process are shown in Fig. 3.5. For, (e) after annealing, (f) after roughening and (g), after etching. Growth of $\sim 12$ mono-layers of $\mathrm{SrRuO}_{3}$ resulted in an atomically smooth film, as shown by the AFM height image and profile in Fig. 3.5(h) and (i). Indicating that the $\mathrm{DyScO}_{3}$ substrate was singly terminated. 


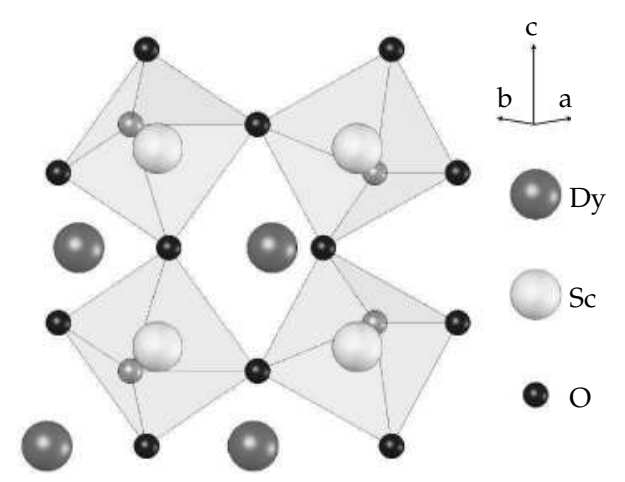

Figure 3.6: Surface structure of $\mathrm{ScO}_{2}$ terminated $\mathrm{DyScO}_{3}$ (110) viewed from above, showing two atomic planes. Image adapted from Kleibeuker et al. ${ }^{[47]}$.

\subsubsection{Structural characterization of $\mathrm{DyScO}_{3}(110)$ surfaces}

Now $\mathrm{DyScO}_{3}$ (110) substrates can be chemically treated independent of the surface configuration after annealing and growth of $\mathrm{SrRuO}_{3}$ on such treated substrates results in atomically smooth films. The exact surface structure of these treated surfaces as well as untreated $\mathrm{DyScO}_{3}$ is not well studied up to now. Therefore, the structure of various states of the $\mathrm{DySCO}_{3}$ surface were studied using RHEED, TOF-MS and SXRD, i.e., as-received, annealed, etched (E), roughened (R) and roughened+etched (RE). A schematic representation of the $\mathrm{DyScO}_{3}$ surface structure is shown in Fig. 3.6 and corresponding bulk room temperature lattice parameters of $\mathrm{DyScO}_{3}$ are given in Table 3.1.

\section{RHEED analysis}

The in-plane surface structure of $\mathrm{DyScO}_{3}$ was studied using reflection high energy electron diffraction (RHEED). Due to the gracing incidence angle of the electron beam and the limited electron escape depth, RHEED is highly surface sensitive. By studying diffraction from topmost atomic layers, the in-plane crystalline ordering of the surface can be determined, see also section 2.2.3.

In Fig. 3.7 RHEED patterns of a chemically treated $\mathrm{DySCO}_{3}(110)$ surface are shown. The images were recorded at background pressure of $10^{-3} \mathrm{mbar}$ at room

Table 3.1: Room temperature fractional coordinates of $\mathrm{DyScO}_{3}$, space group \#62 (Pbnm). ${ }^{[62]}$

\begin{tabular}{cccc}
\hline \hline Atom & $\mathrm{x}$ & $\mathrm{y}$ & $\mathrm{z}$ \\
\hline Dy & 0.0172 & 0.9393 & 0.2500 \\
Sc & 0.0000 & 0.5000 & 0.0000 \\
$\mathrm{O} 1$ & 0.8804 & 0.5550 & 0.2500 \\
$\mathrm{O} 2$ & 0.6926 & 0.3040 & 0.9392 \\
\hline \hline
\end{tabular}



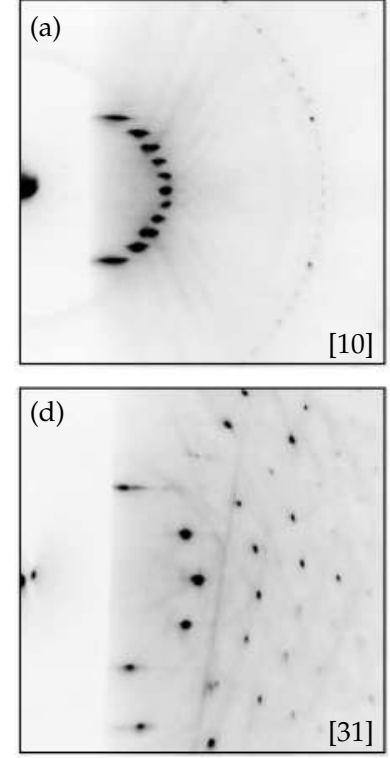
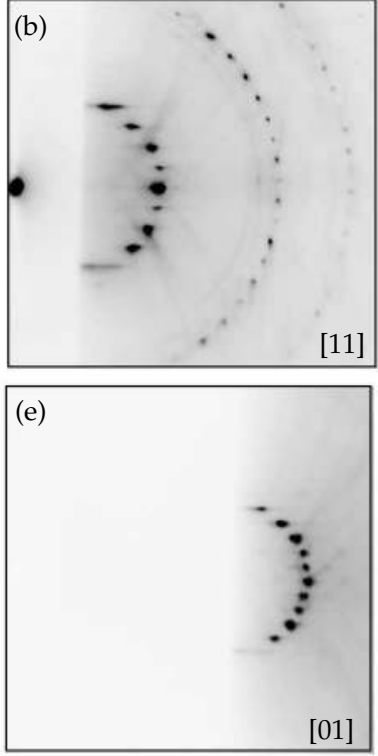
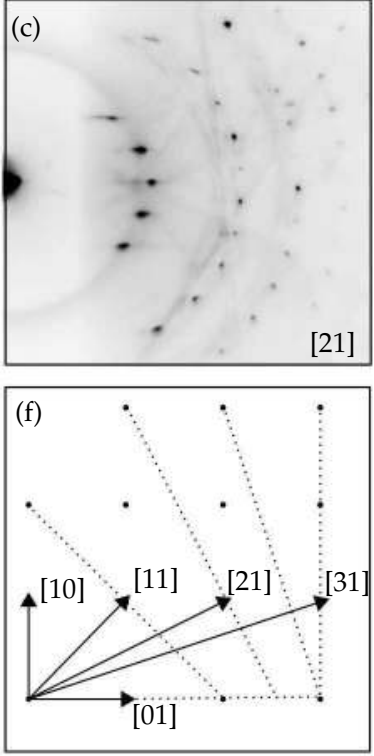

Figure 3.7: RHEED patterns of a $\mathrm{DyScO}_{3}$ (110) surface along different pseudo-cubic inplane crystal directions. Along [01], (a), [11] (b), [21] (c), [31] (d) and along the [01] direction (e). A schematic drawing of these direction is shown in (f).

temperature. ${ }^{5}$ In Fig. 3.7(a-e) RHEED patterns in the [10], [11], [21], [31] and [01] directions are shown. The directions refer to the pseudo-cubic in-plane crystal orientation. The in-plane crystal directions are schematically shown in Fig. 3.7(f). The size of the unit-cell scales inversely with the spacing of the diffraction spots. The observed diffraction patterns correspond the a bulk in-plane orthorhombic $\mathrm{DySCO}_{3}$ unit-cell. The [01] and [10] patterns should not be equal if the true orthorhombic unit-cell of $\mathrm{DyScO}_{3}$ is considered. However, within the experimental resolution, the difference between the [01] and [10] directions could not be distinguished. ${ }^{6}$

The observed diffraction patterns showed a four-fold symmetry and no superstructure was observed. No clear indications of a surface reconstruction were found using RHEED. However, possible variations at the length scale of the unitcell itself cannot be excluded. Diffraction patterns of as-received and annealed $\mathrm{DySCO}_{3}$ substrates could not be distinguished from patterns of the chemically treated substrates.

\footnotetext{
${ }^{5} \mathrm{DyScO}_{3}$ charges during electron illumination, because it is a wide bandgap insulator $(5.9 \mathrm{eV})$. Some oxygen gas was used to neutralize the sample surface.

${ }^{6} \mathrm{~A}$ higher incidence angle was used for measuring the [01] reflection. Part of the sample holder was blocking the electron beam at that specific angle.
} 
(a)
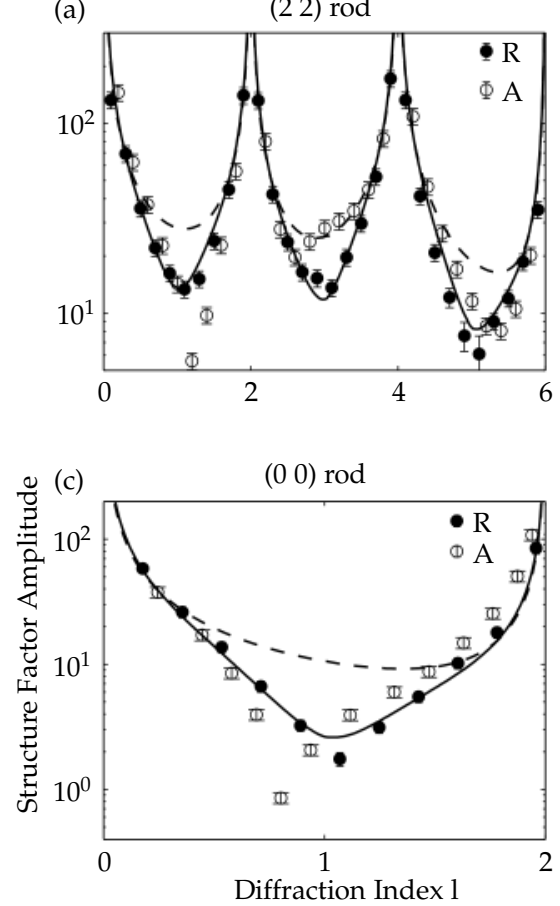

(b)

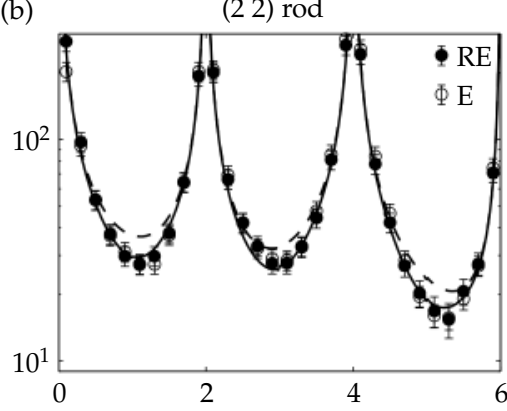

(d)

(0 0) rod

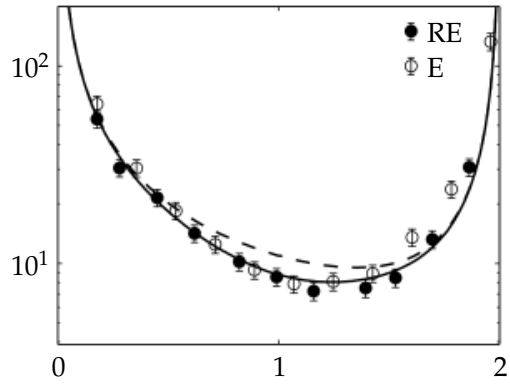

Figure 3.8: Selected CTRs measured by SXRD of $\mathrm{DyScO}_{3}$ after annealing (A) and after surface roughening (R) in (a) and (d). After etching (E) and after roughening+etching (RE) in (b) and (d). The dashed curves are modeled fits for perfect $\mathrm{ScO}_{2}$ terminated surfaces. The solid curves are modeled fits, where the occupancy of the top four surface layers was optimized to fit the experimental data. Adapted from Kleibeuker et al. ${ }^{[47]}$

\section{Surface X-ray diffraction}

SXRD experiments were done on annealed (A), roughened (R), etched $(\mathrm{E})$ and roughened+etched (RE) $\mathrm{DyScO}_{3}$ (110) substrates. SXRD is a highly surface sensitive technique, which can be used to determine the structure of crystal surfaces by measuring the intensity profile of crystal truncation rods (CTR), see also section 2.3.2. ${ }^{[31]}$

The crystallographic directions were defined in such a way that $l$ is in the direction perpendicular to the surface and $h$ and $k$ lie in the surface plane. The bulk $\mathrm{DySCO}_{3}$ atomic position used for modeling of the observed CTRs are given in Table 3.1. The experimental data was fitted by varying the occupancy of each atomic layer taking into account the top four atomic planes, i.e., two DyO layers and two $\mathrm{ScO}_{2}$ layers. All atoms were fixed to their bulk positions. Note that, in the presented model a perfectly $\mathrm{DyO}$ terminated surface will give results equivalent to that of a perfectly $\mathrm{ScO}_{2}$ terminated surface.

Experimental results are shown in Fig. 3.8, along with modeled CTRs for per- 
fectly $\mathrm{ScO}_{2}$ terminated $\mathrm{DyScO}_{3}$ (dashed curves) and optimized models (solid curves). The $\mathrm{A}$ and $\mathrm{R}$ samples did not match the model for perfect $\mathrm{ScO}_{2}$ termination, while the $\mathrm{E}$ and RE samples showed a reasonable agreement with the perfect model. Optimized fit results by changing the occupancy of the top four layers for the $\mathrm{R}$ and $\mathrm{A}$ samples indicated a mixed surface termination ( $\chi^{2}$ equals 3.6 and 3.8 respectively) with a higher DyO occupancy compared to $\mathrm{ScO}_{2}$. Optimized fits of the $\mathrm{E}$ and $\mathrm{RE}$ samples were found for singly terminated $\mathrm{ScO}_{2}$ surfaces with a coverage of 0.90 of the top two atomic layers ( $\chi^{2}$ equals 2.5 and 2.3 respectively). The lowered occupancy of the top most surface layer for $\mathrm{R}$ and RE samples agreed well with the observed AFM results, which indicated unit-cell holes on the terraces. ${ }^{[31,47]}$

The stability of the fit result for the RE sample was tested by applying small variations to the model and measure the effects on the goodness of fit $\left(\chi^{2}\right)$. For example, adding more than four layers to the model did not improve the fit result for the RE sample. Moreover, the fit did not improve by decreasing the coverage of the top two layers for the RE sample. Adding 5\% mixed termination to the RE sample increased $\chi^{2}$ and changing the termination to $\mathrm{DyO}$ with a partial $\mathrm{ScO}_{2}$ coverage reduced the fit result as well. This indicates, the optimized model with a coverage of 0.90 provided a stable fit the experimental data.

SXRD results show that the surfaces obtained for E and RE samples can be well described by using bulk atomic positions. An unreconstructed perfectly $\mathrm{ScO}_{2}$ terminated surface provided the best fit the experimental data. The surfaces of $A$ and $R$ samples were found to be mixed terminated.

\section{Angle resolved mass spectroscopy of recoiled ions}

Angle resolved mass spectra of recoiled ions (AR-MSRI) were recorded on all four different states of $\mathrm{DyScO}_{3}$ at different azimuthal angles, i.e., annealed, etched, roughened and as-received. This time-of-flight based mass spectroscopy technique was used for surface composition analysis, see also section 2.3.4. [63]

In Fig. 3.9 the Sc/Dy ratio of various samples is given as a function of the azimuthal angle. The measured spectra were normalized using the K peak intensity. Clear peaks were observed at $-45^{\circ}$ and $45^{\circ}$ for the chemically etched samples (E, $\mathrm{RE}$ ). This indicated a systematic blocking of Dy ions occurred at these angles, by either Sc or O. This blocking can only occur if $\mathrm{ScO}_{2}$ is the dominant terminating surface plane. Annealed and roughened $\mathrm{DyScO}_{3}$ samples showed no clear dependence of the Sc/Dy ratio as a function of azimuthal angle, indicating a mixed Dy/Sc surface structure. However, the as-received sample showed a similar, but reduced angular dependence compared to the etched samples, indicating a more $\mathrm{ScO}_{2}$ terminated surface prior to annealing. ${ }^{7}$

A four-fold symmetry was found for all samples. Note that, one would expect small changes every 90 degrees, due to the orthorhombic unit-cell of $\mathrm{DyScO}_{3}$.

\footnotetext{
${ }^{7}$ AR-MSRI experiments were performed on different samples, which could have a different initial Sc/Dy ratio. The Sc/Dy ratio of as-received samples was not studied in detail.
} 


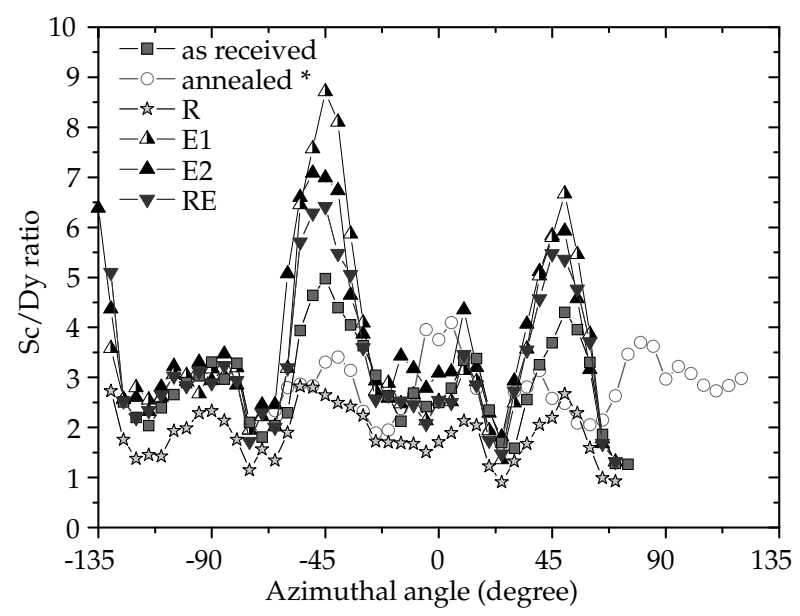

Figure 3.9: Time of flight mass spectroscopy Sc/Dy ratio as a function of azimuthal angle for various $\mathrm{DyScO}_{3}$ substrates, i.e., as-received, annealed, etched, roughened and roughened/etched.

However, similar to RHEED, the accuracy of the experiment is too low to distinguish the different orthorhombic directions and only the pseudo-cubic nature of the structure was visible. Some variations in the peak intensity were observed for various samples. For example, when comparing two etched samples, E1, E2. These variations might be due to differences in surface roughness, which could smear out the intensity of the recoiled species.

Overall, AR-MRSI indicated $\mathrm{ScO}_{2}$ to be the dominant top most surface layer of etched and as-received samples. However, the possible presence of a low fraction of Dy ions at the surface cannot be excluded.

\subsection{Artificially induced DyO termination}

Surfaces with a $\mathrm{ScO}_{2}$ surface termination can now be readily prepared. However, surface with the opposite surface termination (DyO) are also of interest. For example, for studying interface effects in polar oxides. ${ }^{[64]}$ Therefore, the surface termination and structure of well-defined chemically treated $\mathrm{DyScO}_{3}$ (110) was used as a starting point to artificially induce a DyO termination by PLD. The PLD growth of $\mathrm{Dy}_{2} \mathrm{O}_{3}$ was studied in order to artificially induce a change in surface termination, comparable to the growth of $\mathrm{SrO}$ on $\mathrm{TiO}_{2}$ terminated $\mathrm{SrTiO}_{3}$ substrates. ${ }^{[53]}$ The morphology of such DyO films was studied using AFM and the structure was analyzed by SXRD. 


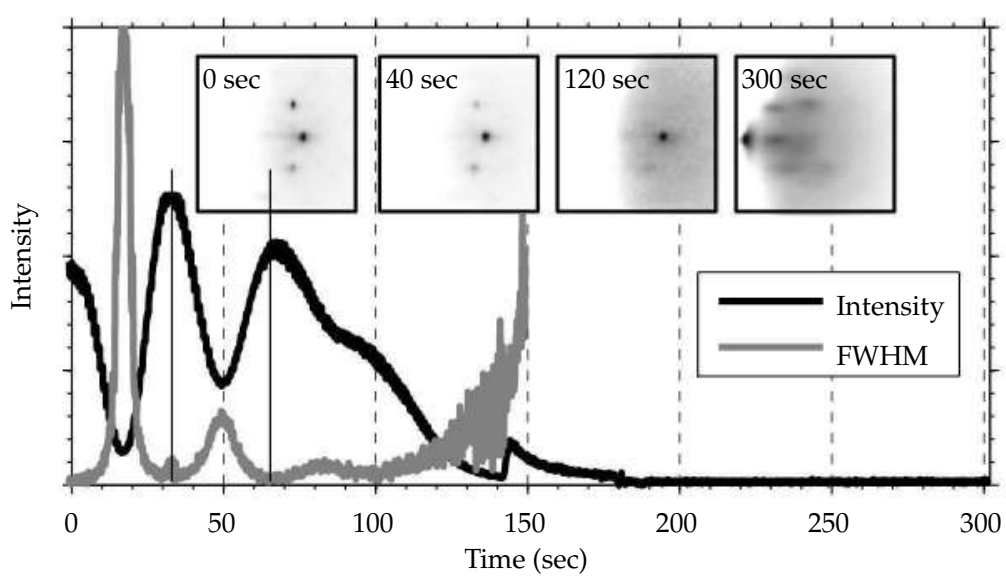

Figure 3.10: RHEED time-evolution of the growth of $\mathrm{Dy}_{2} \mathrm{O}_{3}$ on $\mathrm{DyScO}_{3}$ by PLD. Insets show the RHEED pattern at various times during the deposition.

\section{PLD growth of $\mathrm{Dy}_{2} \mathrm{O}_{3}$ on $\mathrm{DyScO}_{3}(110)$}

By depositing $\mathrm{Dy}_{2} \mathrm{O}_{3}$ on singly $\mathrm{ScO}_{2}$ terminated $\mathrm{DyScO}_{3}(110)$ substrates a switch to a DyO termination is expected. Therefore, first, the growth of $\mathrm{Dy}_{2} \mathrm{O}_{3}$ by PLD was studied using in situ RHEED. In Fig. 3.10 the RHEED time evolution of the specular spot of $\mathrm{Dy}_{2} \mathrm{O}_{3}$ on $\mathrm{DyScO}_{3}$ is shown. Two clear oscillations were observed which occurred every 33 seconds, as indicated by the vertical black lines. The full width half maximum (FWHM) of the specular RHEED spot showed a clear minimum as the peak intensity showed a maximum, indicating completion of one monolayer. The intensity dropped after the first two oscillations and became nearly zero after 150 seconds. By optimizing the tilt angle after growth (300 seconds), a spotty RHEED pattern was found, which indicated a rough surface. $\mathrm{Dy}_{2} \mathrm{O}_{3}$ has a lattice constant of $10.66 \AA$, which corresponds to a strain of $11 \%$ on $\mathrm{DyScO}_{3}$ (110). However, if rotated $45^{\circ}$ the strain is reduced to $4.7 \%$ as is observed for MBE growth on $\mathrm{SrTiO}_{3}{ }^{[65]}$, which also resulted in spotty RHEED patterns and island growth.

After completion of one RHEED oscillation (40 seconds), the diffraction pattern was nearly identical to that of the $\mathrm{ScO}_{2}$ terminated $\mathrm{DyScO}_{3}$ (0 seconds), as shown by the inset in Fig. 3.10. The overall intensity was reduced due to the fact the image was recorded just after the first maximum. This indicates the in-plane surface structure had not changed after the deposition of 33 pulses of $\mathrm{Dy}_{2} \mathrm{O}_{3}$ and DyO adapted to the crystal structure of $\mathrm{DyScO}_{3}$. The second oscillation might be caused by the formation of a Ruddlesden-Popper ${ }^{[66]}$ like defect, where a double stacking of A-site layers occurs. This double A-site layer would also fit to the $\mathrm{DyScO}_{3}$ structure. Upon continuation of the deposition, the RHEED pattern changed to a spotty pattern, indicating a change in crystal structure and/or surface roughness, most likely due to the formation of $\mathrm{Dy}_{2} \mathrm{O}_{3}$. The possible forma- 
tion of $\mathrm{Dy}_{2} \mathrm{O}_{3}$ films is not studied in this work. For the purpose of artificially inducing a DyO surface termination only a single monolayer of DyO should be deposited.

\section{Surface analysis of DyO terminated $\mathrm{DyScO}_{3}$}

A DyO over-layer was deposited using interval-deposition ${ }^{[67]}$ by applying 33 pulses at $50 \mathrm{~Hz}$. The resulting surface morphology is shown in Fig. 3.11(e). An atomically smooth surface with clear terrace steps was observed. Small holes were observed on the terraces, which were $\sim 0.4 \mathrm{~nm}$ deep, while no TM-AFM phase contrast was observed (not shown). Therefore, no experimental evidence for mixed termination was found based on AFM analysis.

RHEED patterns before and after deposition of DyO are given in Fig. 3.11(f). Both showed a clear RHEED pattern with spots indicative of two-dimensional or flat surface. The intensity difference between the central spot and the two symmetric side-spots was reduced after deposition. A similar change in contrast is observed when the surface termination of $\mathrm{SrTiO}_{3}$ is changed ${ }^{8}$. ${ }^{[60]}$ SXRD CTR measurements and models of this film are shown in Fig. 3.11(a-d). The dotted line corresponds to a model with a perfect DyO surface termination. This perfect DyO termination model did not match the experimental data well. However, an optimized model with a DyO coverage of $65 \%$ on a $\mathrm{ScO}_{2}$ terminated $\mathrm{DyScO}_{3}$ substrate gave a reasonable fit, as indicated by the solid-lines.

\subsection{Discussion}

\section{Annealed $\mathrm{DyScO}_{3}$}

After annealing the surface morphology of $\mathrm{DyScO}_{3}$ (110) shows various configuration of (dis)ordered areas of $\mathrm{DyO}$ and $\mathrm{ScO}_{2}$ surface termination. In some cases, areas of different surface termination order along the existing terrace step edges. By careful analysis of the AFM height and friction/phase images the surface termination configurations can be classified. Most substrates showed steps of 1.0 u.c. in height and no friction/phase contrast, indicating single termination. However, structural analyses of annealed $\mathrm{DyScO}_{3}$ substrates indicated a mixed surface terminated, see section 3.3. Therefore, AFM is not suitable to proof single termination. However, when height and/or friction/phase contrast is observed by AFM the surface is mixed terminated and the termination configuration can be determined. Both configurations for ordered mixed termination along terrace steps depicted in Fig. 3.2(c,d) are experimentally observed, although they cannot unambiguously be distinguished from randomly ordered mixed terminated models (e) and (f) in all cases. The increased surface roughness on part of the terraces, observed in Fig. 3.3(a) does show that model Fig. 3.2(e) and (f) are

\footnotetext{
${ }^{8}$ The spot doubling observed on some spots was probably caused by an instrumental effect.
} 

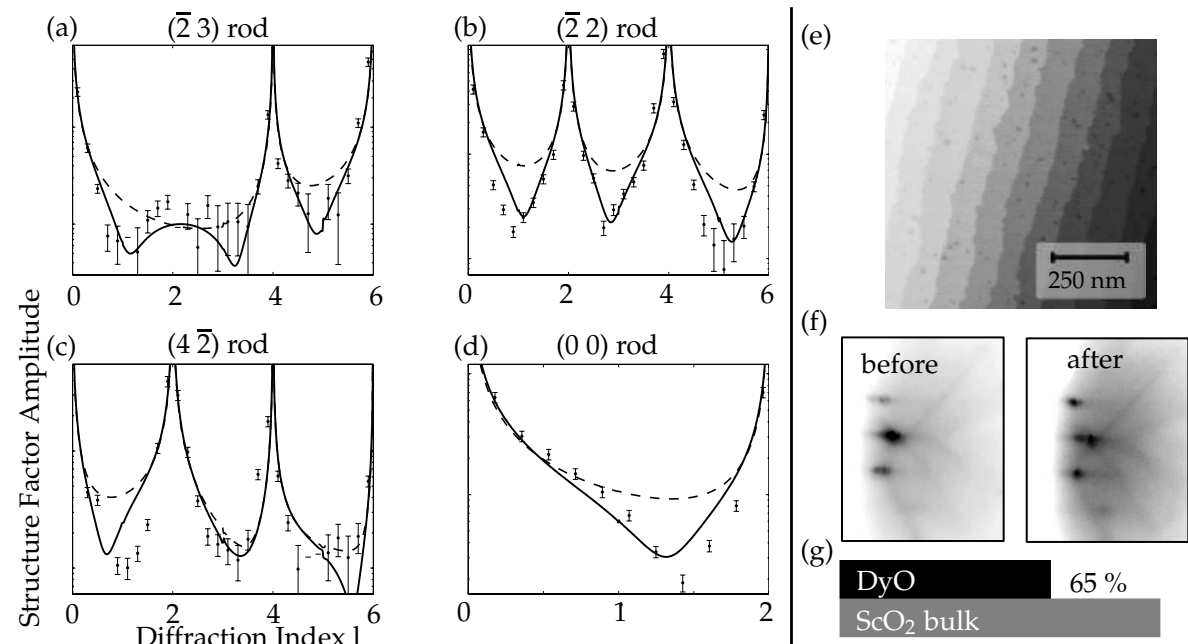

Figure 3.11: SXRD results (a-d) of a PLD grown DyO overlayer on $\mathrm{DyScO}_{3}$. Datapoints are given with corresponding errorbars, the solid lines are based on a model fit with $65 \%$ coverage of $\mathrm{DyO}$ on $\mathrm{ScO}_{2}$ terminated surface. The dashed lines correspond to a perfect $\mathrm{DyO}$ termination. In (e) an AFM image of the studied sample is shown and in (f) the RHEED pattern prior to and after growth of DyO are shown. A schematic of the $65 \%$ model fit is given in (g). SXRD experiments and analyses were done by Paul Tinnemans. ${ }^{[31]}$

likely to occur. The exact termination pattern should be considered when discussing growth and properties of thin films grown on patterned surface terminations. ${ }^{[57,58]}$ Ordered mixed terminated substrates are selected based on AFM analyses and can be used to create $\mathrm{SrRuO}_{3}$ nanostructures, as shown in chapter 4.

Dirsyte et al. ${ }^{[68]}$ suggest a transition from a dominant DyO termination after 30-60 minutes of annealing to $\mathrm{ScO}_{2}$ after 10 hours of annealing in oxygen or argon atmosphere. However, in this chapter, most ordered mixed termination patterns were observed after 30 minutes to 4 hours of annealing, but ordered mixed termination was also found after 12 hours of annealing. Moreover, ARMSRI results indicate a dominant $\mathrm{ScO}_{2}$ termination is present on annealed and as-received substrates which does not seem to match the observations of Dirsyte et al. ${ }^{[68]}$ Simultaneously annealed substrates show different types of termination ordering. It appears as if the initial surface configuration of $\mathrm{DyScO}_{3}$ varies for each substrate, resulting in a range of different configurations after annealing.

\section{$\mathrm{DyScO}_{3}$ surface structure}

To control the surface termination of $\mathrm{DyScO}_{3}$, a chemical etching process was developed. The DyO at the surface can be selectively removed to yield singly $\mathrm{ScO}_{2}$ terminated $\mathrm{DyScO}_{3}(110)$ substrates. The surface morphology studied using AFM after chemical etching did not show any indication of mixed surface termi- 
nation. However, in some cases growth of $\mathrm{SrRuO}_{3}$ on treated $\mathrm{DyScO}_{3}$ indicated the surface was not completely single terminated, as shown in Fig. 3.5. Therefore, a surface roughening step was introduced, which increased the etching rate. Moreover, this enhanced etching rate, reduced the influence of the exact surface configuration obtained after annealing. Effectively, reducing the time required to obtain complete single termination during $\mathrm{NaOH}$ etching.

These well-defined singly terminated $\mathrm{DyScO}_{3}$ substrates are used to study the surface structure of $\mathrm{DyScO}_{3}$ using AR-MSRI, RHEED and SXRD. Experiments agreed well with a $\mathrm{DyScO}_{3}$ surface structure which is bulk-like and singly terminated. However, a bulk-like surface termination would result in a polar surface. Any possible reconstruction seemed to occur within one $\mathrm{DyScO}_{3}$ orthorhombic unit-cell since no additional diffraction peaks were observed in RHEED and SXRD experiments. In the case of single $\mathrm{ScO}_{2}$ termination the charge on the surface could be reduced to $-1 / 2$ by removing one $\mathrm{O}$ atom, resolving the polar surface-vacuum interface.

\section{Artificial DyO termination}

Well-controlled formation of complete A-site surface termination was tested by depositing DyO using PLD on singly terminated $\mathrm{DyScO}_{3}$ substrates. The resulting DyO films had a surface morphology and roughness similar to that of the substrate. RHEED patterns before and after deposition of a single DyO layer showed a similar crystal structure. However, a small intensity change is observed between the main peak and the symmetrical side peaks. The side peaks became more pronounced after deposition of DyO. A similar effect is observed for $\mathrm{SrO}$ terminated $\mathrm{SrTiO}_{3} .{ }^{[60]}$ Since the RHEED pattern remains two-dimensional in nature, showing peaks aligned on a Laue circle, the DyO overlayer appears to adapt to the $\mathrm{DyScO}_{3}$ crystal structure.

To confirm that this DyO overlayer adapts to the $\mathrm{DyScO}_{3}$ structure, it was studied using SXRD. The experimental data of deposited Dy overlayer was successfully fitted using a DyO termination model with a coverage of $65 \%$. However, a coverage close to unity was expected. Possibly the number of applied pulses by PLD was too low. ${ }^{9}$ The number was estimated using previously observed growth speeds. Moreover, the sample used for SXRD was grown using interval-deposition, which did not allow for live RHEED monitoring of the specular intensity. No signs of a surface reconstruction of DyO overlayers was found. Resolving the polar nature of the $\mathrm{DyScO}_{3}$ surface for DyO terminated surfaces would require Dy vacancies, one in every six pseudo-cubic surface unit-cells (two in every three orthorhombic). This model is not compatible with the diffraction experiments, as no systematic cation vacancies were observed.

\footnotetext{
${ }^{9}$ Due to the limited possibilities to perform SXRD experiments no attempts were made to repeat this experiment with a larger number of DyO pulses.
} 


\subsection{Conclusions}

After annealing, various $\mathrm{DyScO}_{3}$ (110) surface morphologies and surface termination configurations are obtained. No clear dependence of the anneal time on the mixed termination configuration was found, apart from the straightening step terrace features with increasing anneal time. An unknown variation in the $\mathrm{DySCO}_{3}$ surface termination configuration prior to annealing is likely the main reason for the range of observed termination configurations after annealing. Ordered mixed terminated substrates can be selected based on AFM analysis.

The surface of selectively etched $\mathrm{DyScO}_{3}$ substrates studied by RHEED, SXRD and AR-MSRI has a dominant $\mathrm{ScO}_{2}$ surface termination. $\mathrm{SrRuO}_{3}$ growth experiments indicate complete single termination can be achieved. An additional surface roughening step enhances the selective etching rate, which strongly reduces any influence of the initial surface termination ordering on the selective etching. No structural evidence for surface reconstructions or displacements of polar $\mathrm{ScO}_{2}$ termination is found. Suggesting the polar surface is relieved by introducing oxygen vacancies in the topmost $\mathrm{ScO}_{2}$ layer.

A DyO over layer was prepared on $\mathrm{ScO}_{2}$ terminated substrates which adapts to the $\mathrm{DyScO}_{3}$ crystal structure when the thickness is below 2 unit-cell. SXRD experiments indicated a $65 \%$ coverage of $\mathrm{DyO}$, which was well described by a bulk-like surface termination model, e.g., no atomic displacements. Moreover, RHEED patterns similar to the $\mathrm{ScO}_{2}$ terminated surface were obtained. The DyO over-layer is formed as part of the bulk $\mathrm{DyScO}_{3}$ structure. How the polar discontinuity is resolved on $\mathrm{DyO}$ is unclear, but no indications of ordered cation vacancies are found. 


\title{
Chapter 4
}

\section{Self-organization on ordered oxide surface terminations}

\begin{abstract}
A method of fabricating oriented single-crystalline $\mathrm{SrRuO}_{3}$ nanostructures using a bottom-up approach relying on diffusion-mediated selforganization is demonstrated. $\mathrm{DyScO}_{3}$ (110) substrates exhibiting an ordered striped phase of $\mathrm{DyO}$ and $\mathrm{ScO}_{2}$ chemical termination are used as a template for pulsed laser deposition growth of $\mathrm{SrRuO}_{3} . \mathrm{SrRuO}_{3}$ preferentially nucleates on the $\mathrm{ScO}_{2}$ termination. The resulting nanowires are single crystalline, conducting and isolated from each other, typically $100 \mathrm{~nm}$ wide and 5-10 $\mathrm{nm}$ high. The resulting patterns are characterized using AFM, XRD and SEM and the stacking sequence and interface configurations are studied using HAADF TEM and STEM EELS.
\end{abstract}

Part of the work in this chapter is published in: B. Kuiper, J.L. Blok, H.J.W. Zandvliet, D.H.A. Blank, G. Rijnders, and G. Koster. MRS Communications 1, 17 (2011). ${ }^{[57]}$ 


\subsection{Introduction}

Thin film deposition techniques of perovskite-type oxides, that allow for atomic control, have been used to build artificial crystal structures bottom-up. By stacking unit-cell-high layers of different materials on top of each other to create vertically layered (two-dimensional) structures, new and appealing functionalities are observed. For example, by creating a conducting interface between two insulating materials, ${ }^{[10]}$ enhancement of ferroelectricity by breaking symmetry ${ }^{[69]}$ or superconductivity when creating a superlattice of $\mathrm{BaCuO}_{2} /(\mathrm{Sr}, \mathrm{Ca}) \mathrm{CuO}_{2} \cdot{ }^{[70]}$

To understand how-to achieve atomic control in such complex-oxides, it is helpful to represent the perovskite $\mathrm{ABO}_{3}$ crystal by alternating planes of $\mathrm{AO}$ and $\mathrm{BO}_{2}$, as depicted in Fig. 4.1(a). Both types of layer can be present at the surface of a $(001)_{\mathrm{pc}}$-cut single crystal. To date, a great deal of effort has been put into (chemical) methods to obtain starting surfaces with a single termination ${ }^{[25,26,47]}$ (i.e., either $\mathrm{AO}$ or $\mathrm{BO}_{2}$ ), which are optimal for two-dimensional, vertical structures.

In an interesting case of $\mathrm{SrRuO}_{3}$, the termination switches from B-site to Asite during film growth, while a correlated change in kinetic growth mode is observed. ${ }^{[71]}$ Therefore, a scenario as sketched in Fig. 4.1(b), where both terminations are present at the surface could offer interesting possibilities for both vertical and lateral kinetically controlled crystal growth. In-plane control of atomic ordering in artificial crystal structures can lead to a whole host of new possibilities to create materials with new or enhanced functionality. Moreover, one could exploit the mixed termination to fabricate epitaxial low-dimensional structures such as nanowires and nanodots bottom-up.

Bottom-up fabrication of nanostructures has proven successful in creating magnetic/ferroelectric columnar matrices of $\mathrm{BiFeO}_{3}-\mathrm{CoFe}_{2} \mathrm{O}_{4} \cdot{ }^{[17,72]}$ By using selfassembly during epitaxial growth one can create nanostructures without the need of physical or chemical etching, which can damage the surface structure, opening new possibilities to study size effects in oxide systems. ${ }^{[16]} \mathrm{SrRuO}_{3}$ is a commonly used electrode material in oxide heterostructures and is also studied for its interesting electrical and magnetic properties as it is one of the very few undoped conducing perovskite oxides. Effects of size reduction on intrinsic $\mathrm{SrRuO}_{3}$ properties as well as electrode induced size effects are used to tune properties of thin films, as discussed in chapter 7.

Two achieve this epitaxial self-organization, two prerequisites have to be fulfilled: (i) the substrate must show an ordered distribution of $\mathrm{AO}$ and $\mathrm{BO}_{2}$ terminated domains and (ii) subsequent heteroepitaxy must be sensitive to the chemical nature of the termination. Yet, to date not many systems have been shown to exhibit such ordered termination, but rather a random distribution of the two possible terminations. ${ }^{[60]}$ In some cases, the minority termination (after chemical etching) appears to be stabilized by the preexisting vicinal steps on the surface. Subsequent heteroepitaxy of metallic and magnetic $\mathrm{SrRuO}_{3}$ on these surfaces exhibits a morphology with trenches or elongated islands, clearly correlated with the underlying substrate vicinal steps. ${ }^{[73-75]}$ 


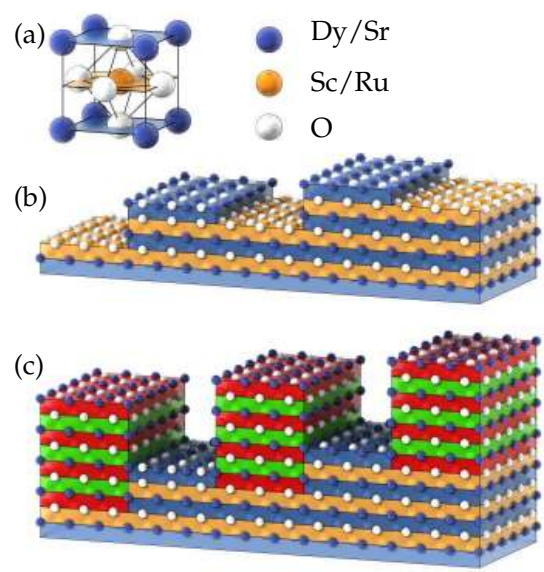

Figure 4.1: Schematic drawing of a perovskite crystal. (a) Cubic perovskite unit-cell. (b) A vicinal and ordered mixed terminated surface. (c) The resulting morphology after growth of a termination sensitive material on a mixed terminated substrate.

The surface termination of $\mathrm{DyScO}_{3}$ can be well-defined and ordered, either singly terminated ${ }^{[26,47]}$ or orderly patterned, as discussed in chapter 3 . Such $\mathrm{DyScO}_{3}$ substrates can act as templates for self-organized epitaxial $\mathrm{SrRuO}_{3}$ nanostructures, schematically drawn in Fig. 4.1(c). Observations of $\mathrm{SrRuO}_{3}$ self-organization during growth are reported on $\mathrm{SrTiO}_{3}{ }^{[73,74,76]}, \mathrm{LaAlO}_{3}{ }^{[75]}$ and $\mathrm{LSAT}^{[77]}$. Initially, the growth of these ordered morphologies on $\mathrm{SrTiO}_{3}$ substrates was attributed to the substrate step edges. Recently, this vision was revised and the formation of a correlated morphology on $\mathrm{SrTiO}_{3}$ is explained by the difference in adatom sticking coefficient between both surface terminations. ${ }^{[58]}$ The latter explanation assumes surface mixed termination to drive the $\mathrm{SrRuO}_{3}$ nanowire formation. However, in this work no clear difference in sticking was observed.

In this chapter, the mechanism driving the $\mathrm{SrRuO}_{3}$ self-organization is studied on $\mathrm{DyScO}_{3}$ templates. The role of step edges, the sticking coefficient, adatom mobility and nucleation rates on $\mathrm{SrRuO}_{3}$ self-organization are studied. The evolution of the nanowire growth is monitored during PLD growth using in situ RHEED and by studying the morphology of the nanowires using atomic force microscopy (AFM). The structure and atomic stacking at the substrate-film interface are revealed using X-ray diffraction and electron microscopy. Moreover, the local properties are explored using conductive AFM experiments.

\subsection{Self-organization and growth of $\mathrm{SrRuO}_{3}$ on mixed terminated surfaces}

In this section the experimental results of the self-organization of $\mathrm{SrRuO}_{3}$ in ordered mixed terminated $\mathrm{DyScO}_{3}$ (110) substrates are presented. 

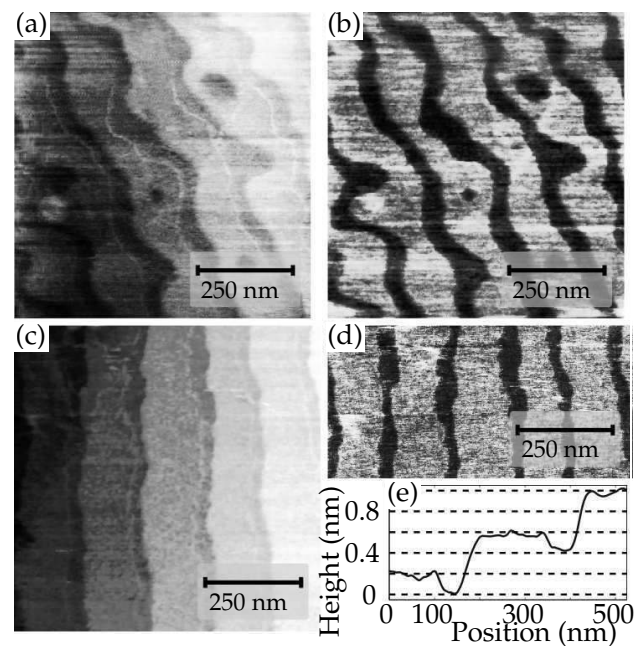

Figure 4.2: $\mathrm{AFM}$ images of doubly terminated $\mathrm{DyScO}_{3}$ substrates. (a) Height and (b) friction force of $\mathrm{DyScO}_{3}$ annealed for 30 minutes and (c) and (d) of $\mathrm{DyScO}_{3}$ annealed for 4 hours. (e) A line profile of (c) with $0.2 \mathrm{~nm}$ spaced dotted lines as guides to the eye.

\subsubsection{Ordered mixed terminated $\mathrm{DyScO}_{3}$ substrates}

The morphology of two $\mathrm{DyScO}_{3}$ (110) substrates with different relative termination domain populations of both surface terminations is shown in Fig. 4.2. Most terraces were about $200 \mathrm{~nm}$ wide and showed $\sim 50 \mathrm{~nm}$ wide areas offset by half a unit-cell. This offset is clearly visible in the AFM line profile, shown in Fig. 4.2(e). The horizontal lines are spaced by $0.2 \mathrm{~nm}$ as guides to the eye, indicating $0.2 \mathrm{~nm}$ and $0.6 \mathrm{~nm}$ steps, 0.5 and 1.5 unit-cell respectively. These offset areas also gave rise to a strong contrast in the simultaneously recorded friction force images, depicted in Fig. 4.2(b,d). This contrast indicated a local difference in tip-surface interaction strength. This combination of height offset and friction force contrast indicated two different surface terminations, i.e., DyO and $\mathrm{ScO}_{2}$ were present at the surface in an ordered fashion. The substrate depicted in Fig. 4.2(a) was annealed for 30 minutes, the step edges were curved and islands of different terminated regions were still present on the terrace. A crystal annealed for 4 hours, depicted in Fig. 4.2(c), showed well-ordered areas of mixed termination with a slightly different ratio between $\mathrm{DyO}$ and $\mathrm{ScO}_{2}$ compared to the sample annealed for 30 minutes. A more detailed overview of various mixed terminated $\mathrm{DyScO}_{3}$ (110) substrates is given in chapter 3.

\subsection{2 $\mathrm{SrRuO}_{3}$ growth and RHEED analysis}

During deposition of $\mathrm{SrRuO}_{3}$ on mixed terminated $\mathrm{DyScO}_{3}$ substrates, the surface structure was studied using in situ RHEED. The specular intensity was monitored over time and diffraction patterns were recorded before, after and during 

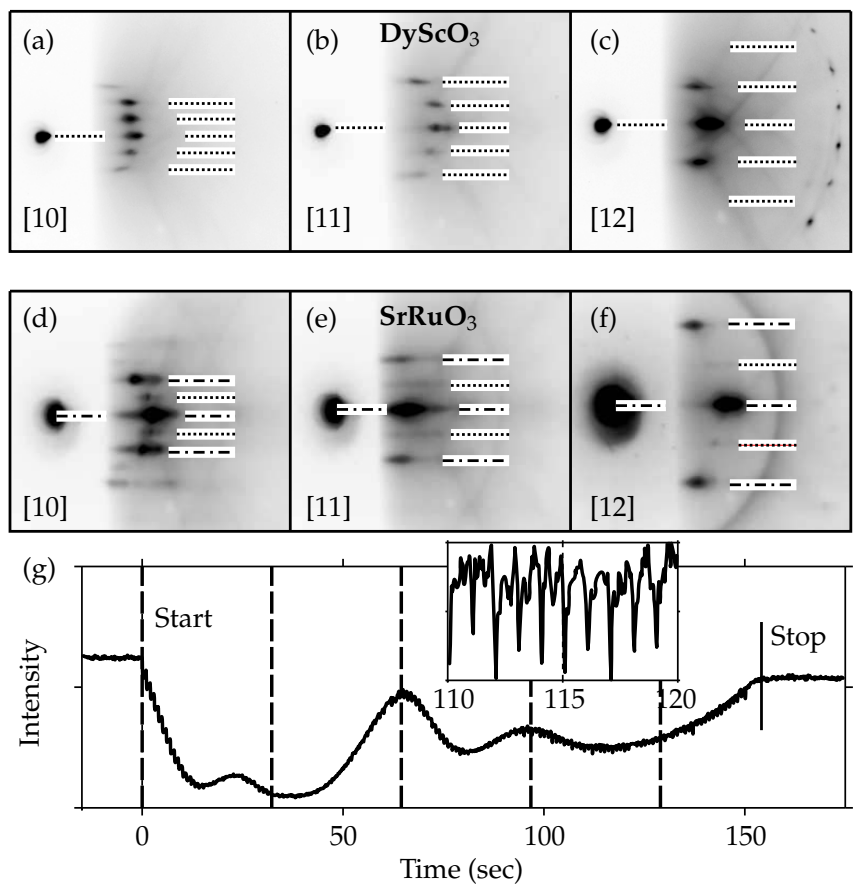

Figure 4.3: RHEED images of (a-c) $\mathrm{DyScO}_{3}$ substrate before deposition of $\mathrm{SrRuO}_{3}$ and (df) after deposition of $\mathrm{SrRuO}_{3}$. The [10], [11] and [12] crystalline orientations are shown, all indicating a two-dimensional RHEED pattern. The dotted lines are guides to the eye, indicating the vertical spacing of the RHEED spots. In $(\mathrm{g})$ the time evolution of the specular intensity of $\mathrm{SrRuO}_{3}$ growth on mixed $\mathrm{DyScO}_{3}$ is shown.

deposition. Such diffraction patterns are shown in Fig. 4.3 for an ordered mixed terminated $\mathrm{DyScO}_{3}$ substrate and for the $\mathrm{SrRuO}_{3}$ nanostructure. Diffraction patterns for three different in-plane directions are shown and labeled with their respective pseudo-cubic [hk] values. $\mathrm{All} \mathrm{SrRuO}_{3}$ patterns shown in Fig. 4.3(d-f) have diffraction spots which lie on a Laue circle. Indicating the spots are formed by reciprocal lattice rods intersecting the Ewald sphere ${ }^{1}$, as discussed in section 2.2.3. Such spots are only clearly visible on atomically smooth surfaces. However, some low intensity additional spots and slight peak broadening were observed. These stripes and spots indicated the nanostructures surface was slightly disordered. ${ }^{[29,78]}$

The RHEED patterns of $\mathrm{SrRuO}_{3}$ had a larger (vertical) spacing between intense diffraction spots, compared to the $\mathrm{DyScO}_{3}$ substrate, especially in the [11] and [12] directions. This spacing is inversely proportional to the unit-cell size. The $\mathrm{DyScO}_{3}$ surface structure is roughly $8 \times 8 \AA^{2}$. Compared to cubic $\mathrm{SrTiO}_{3}(4 \times 4$ $\AA^{2}$ ) the $\mathrm{DyScO}_{3}$ diffraction pattern is expected to have half the peak spacing. In

\footnotetext{
${ }^{1}$ The circular line shape observed in Fig. 4.3(d-f) was caused by a reflection in the RHEED camera set-up, not by the sample.
} 
Fig. 4.3 $\mathrm{SrRuO}_{3}$ dot spacing thus resembles are more cubic like structure compared to the $\mathrm{DyScO}_{3}$ substrate, as indicated by the dotted lines.

Time evolution of the specular RHEED intensity of the growth of 150 pulses of $\mathrm{SrRuO}_{3}$ on ordered mixed terminated $\mathrm{DyScO}_{3}$ is shown in Fig. 4.3(g). A clear intensity maximum was observed after 64 seconds of deposition and an additional maximum after 96 seconds. Dotted vertical lines indicate time intervals of 32 seconds after the start of the deposition. The growth speed was determined at 32 pulses per monolayer after completion of the first oscillation. The first oscillation required twice as much time as the second oscillation. A similar time evolution during $\mathrm{SrRuO}_{3}$ growth is observed for growth on $\mathrm{SrTiO}_{3} \cdot{ }^{[71]}$ This longer first oscillation is attributed to a termination conversion from $\mathrm{TiO}_{2}$ to $\mathrm{SrO}$ instead of having $\mathrm{RuO}_{2}$ as a terminating layer. The time required to complete the termination conversion is equal to the time required to deposit an amount of material equal to two unit-cell layers of $\mathrm{SrRuO}_{3}$. Therefore, the initial oscillation is twice as long as the second. ${ }^{2}$

After the second oscillation, the overall intensity of the specular reflection slowly increased over time, but no additional oscillations were observed. This overall intensity increase was observed for $\mathrm{SrRuO}_{3}$ films grown on $\mathrm{DyScO}_{3}$. However, no such increase was reported on $\mathrm{SrTiO}_{3}$ in the literature. $\mathrm{DyScO}_{3}$ being a wide band gap insulator exhibits surface charging when exposed to high energy electrons in vacuum conditions, hampering RHEED analysis. The conductive $\mathrm{SrRuO}_{3}$ film resolved this surface charging effect. Combined with a change in structure factor this could possibly cause a change in overall RHEED intensity, which could scale with the $\mathrm{SrRuO}_{3}$ coverage or the $\mathrm{SrRuO}_{3}$ thickness (conductivity). During the growth every laser pulse gave rise to a drop in RHEED intensity, followed be a recovery before the next pulse arrived, as is shown in the inset in Fig. 4.3(g). This pulse recovery was strongest after the completion of the first two layers of $\mathrm{SrRuO}_{3}$ and is indicative of a steady-state or step-flow like growth mode.

The small intensity increase after roughly 20 seconds of deposition is not well understood. Similar low intensity oscillations were observed in other samples during the growth of self-organized $\mathrm{SrRuO}_{3}$ on $\mathrm{DyScO}_{3}$. RHEED patterns of $\mathrm{SrRuO}_{3}$ nanostructures are comparable to RHEED patterns of $\mathrm{SrRuO}_{3}$ thin films (not shown). Overall, RHEED indicates, the top surface of the structures are atomically flat when viewed at gracing angle.

\subsubsection{Nanowire formation on $\mathrm{DyScO}_{3}$ substrates}

The substrate templates depicted in Fig. 4.2(a,c) were used as a starting template for growth of $\mathrm{SrRuO}_{3}$. After growth of 300 pulses ${ }^{3}$ of $\mathrm{SrRuO}_{3}$ by PLD on these doubly terminated $\mathrm{DyScO}_{3}$ substrates, the template shape was maintained and

\footnotetext{
${ }^{2}$ Depending on the growth conditions, the termination conversion can take place during deposition of more than two unit-cell layers.

${ }^{3} \mathrm{PLD}$ growth of $\mathrm{SrRuO}_{3}$ is performed at $1 \mathrm{~Hz}$, the completion of one monolayer required roughly 30 pulses.
} 


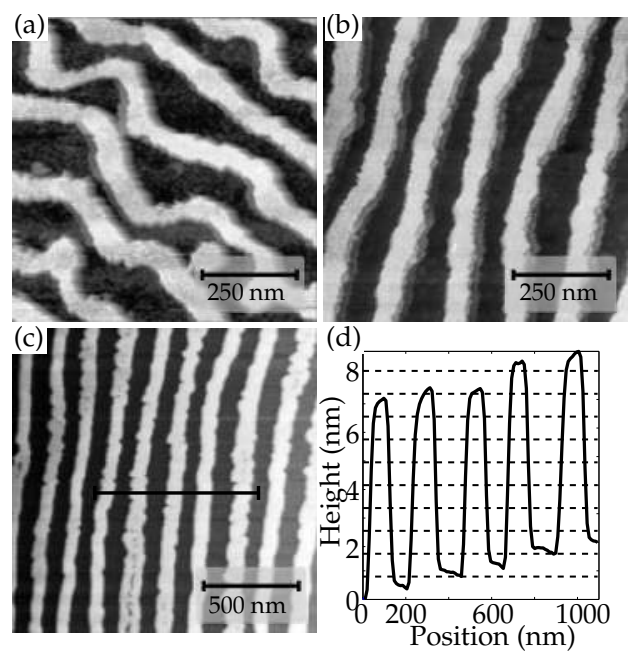

Figure 4.4: $\mathrm{AFM}$ height of (a), (b) $\mathrm{SrRuO}_{3}$ nanostructures after PLD growth on the $\mathrm{DyScO}_{3}$ substrates shown in Fig. 4.2(a) and (c) respectively. In (c) another $\mathrm{SrRuO}_{3}$ nanowire pattern is shown and a corresponding height profile in $(\mathrm{d})$.

multiple unit-cell high nanowires were formed. Figure 4.4(a,b) show that the $\mathrm{SrRuO}_{3}$ morphology reflected the original substrate termination. Wires grew on one of the terminations, while the other termination remained uncovered. Moreover, $\mathrm{SrRuO}_{3}$ columns that grew on circular shaped terraces of the substrate are shown in Fig. 4.2(a). Figure 4.4(c) shows that if the substrate mixed termination was well ordered, the growth resulted in arrays of well-defined nanowires. $\mathrm{SrRuO}_{3}$ mimicked the original surface termination during growth, resulting in an array of nanowires.

The observed wire patterns had a very high aspect ratio as is shown in Fig. 4.5 using a scanning electron microscope (SEM). The wires were well-separated over a length of $15 \mu \mathrm{m}$, but likely run across the entire $5 \times 5 \mathrm{~mm}$ substrate. The $\mathrm{SrRuO}_{3}$ nanowires were up to $8 \mathrm{~nm}$ or 20 mono-layer in height after PLD growth of 300 pulses. The growth speed of $\mathrm{SrRuO}_{3}$ at the given conditions was around 30 pulses per monolayer, so one would expect a $4 \mathrm{~nm}$ thin film if the substrate was singly terminated. Interestingly, the volume of the $8 \mathrm{~nm}$ high nanowires matched the volume of a $4 \mathrm{~nm}$ smooth film, since the coverage was roughly $50 \%$. All the deposited material sticked to the surface and accumulated in the nanowire structures. Therefore, the growth process involved must be diffusion related. No relationship with the substrate step edges or a difference in sticking coefficient was observed, as suggested in literature. ${ }^{[58,74]}$

\section{Substrate influence and thickness evolution}

A diffusion related growth mechanism for the formation of the wire patterns discussed above requires ad-atom transport with a diffusion length $l_{d}$ which is larger 

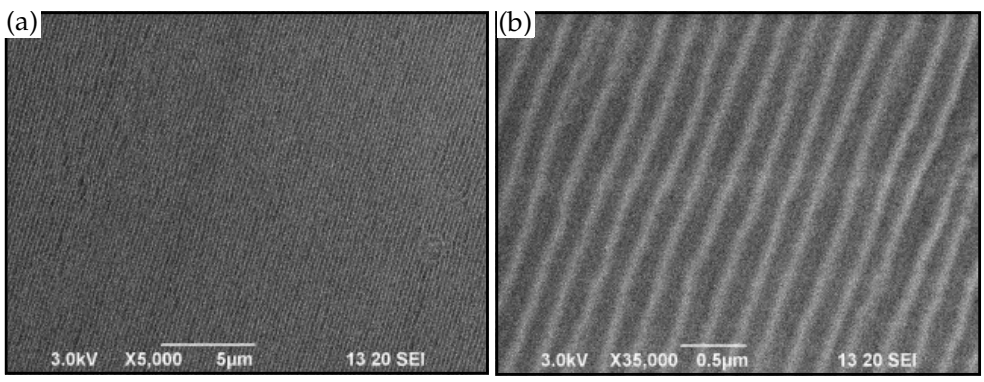

Figure 4.5: Scanning electron microscope (SEM) images of the $\mathrm{SrRuO}_{3}$ nanowire pattern shown in Fig. 4.4(b). (a) overview image $\sim 20 \times 15 \mu \mathrm{m}$ and (b) zoom of $\sim 3 \times 2.5 \mu \mathrm{m}$.

than the separation of the mixed terminated surfaces areas $1_{t}^{4}$ By changing the terrace width, $l_{t}$ and studying the resulting nanostructures such diffusion phenomena can be studied. $\mathrm{A} \mathrm{DyScO}_{3}$ substrate polished with a bow shaped surface height distribution was used to study the effects of miscut/terrace-width on the resulting $\mathrm{SrRuO}_{3}$ features, i.e., nanowires or nanodots. By using one substrate with a local substrate miscut variation, it is possible to study the effects of miscut while using exactly the same growth conditions for each miscut angle. AFM phase and height images of the mixed terminated $\mathrm{DyScO}_{3}$ substrate are shown in Fig. 4.6(a,b) and a schematic of the bow shaped surface in Fig. 4.6(c). The substrate AFM image, shown in (a) and (b), shows a step-width of $320 \mathrm{~nm}$ and two distinct termination areas of roughly $160 \mathrm{~nm}$ and $80 \mathrm{~nm}$ in width were observed.

Figure 4.6(d) shows the formation of wire shaped features on a low terracewidth area, i.e., high miscut angle. No features were observed in between the nanowires, which were roughly the same width as the step-width of the substrate. Upon increasing the terrace-width, as depicted in Fig. 4.6(e,f), the wires had a feature-width smaller than the terrace-width. Moreover, some features were observed in between the nanowires, which are of lower height. Upon further increase of the terrace width, as shown in Fig. $4.6(\mathrm{~g})$, also islands and circles formed, which had a feature-width comparable to the wire shapes.

The experimentally observed relationship between the step-width and the feature-size (wire-width) is shown in Fig. 4.6(h). When increasing the step-width the wire separation increased linearly until roughly $200 \mathrm{~nm}$. Further increasing the step-width did not result in larger nanowires/features. A typical feature size of $170 \mathrm{~nm}$ in width was found for this specific sample.

The feature height after deposition of 660 pulses $^{5}$ is roughly $2 \mathrm{~nm}$ and roughly $4 \mathrm{~nm}$ after 1320 pulses as indicated in Fig. 4.6(i). This in agreement with the growth speed estimated from RHEED ${ }^{6}$. Examples of the surface morphology af-

\footnotetext{
${ }^{4}$ Separation of mixed terminated areas is considered to be equal to the terrace width.

${ }^{5}$ In this experiment a smaller laser spot-size on the $\mathrm{SrRuO}_{3}$ target was used, resulting a reduced growth speed

${ }^{6}$ Here the volume of materials in the main features equals the volume of a similar continuous film, as material also nucleates on other areas. This hampers analysis of the material volume by AFM.
} 

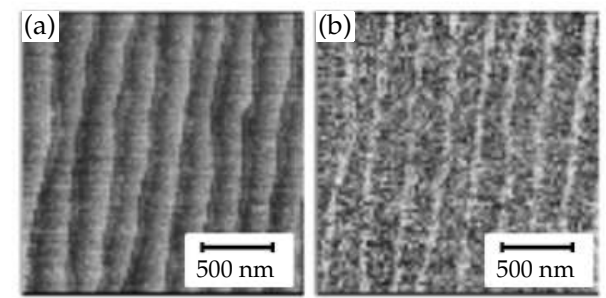

(c)
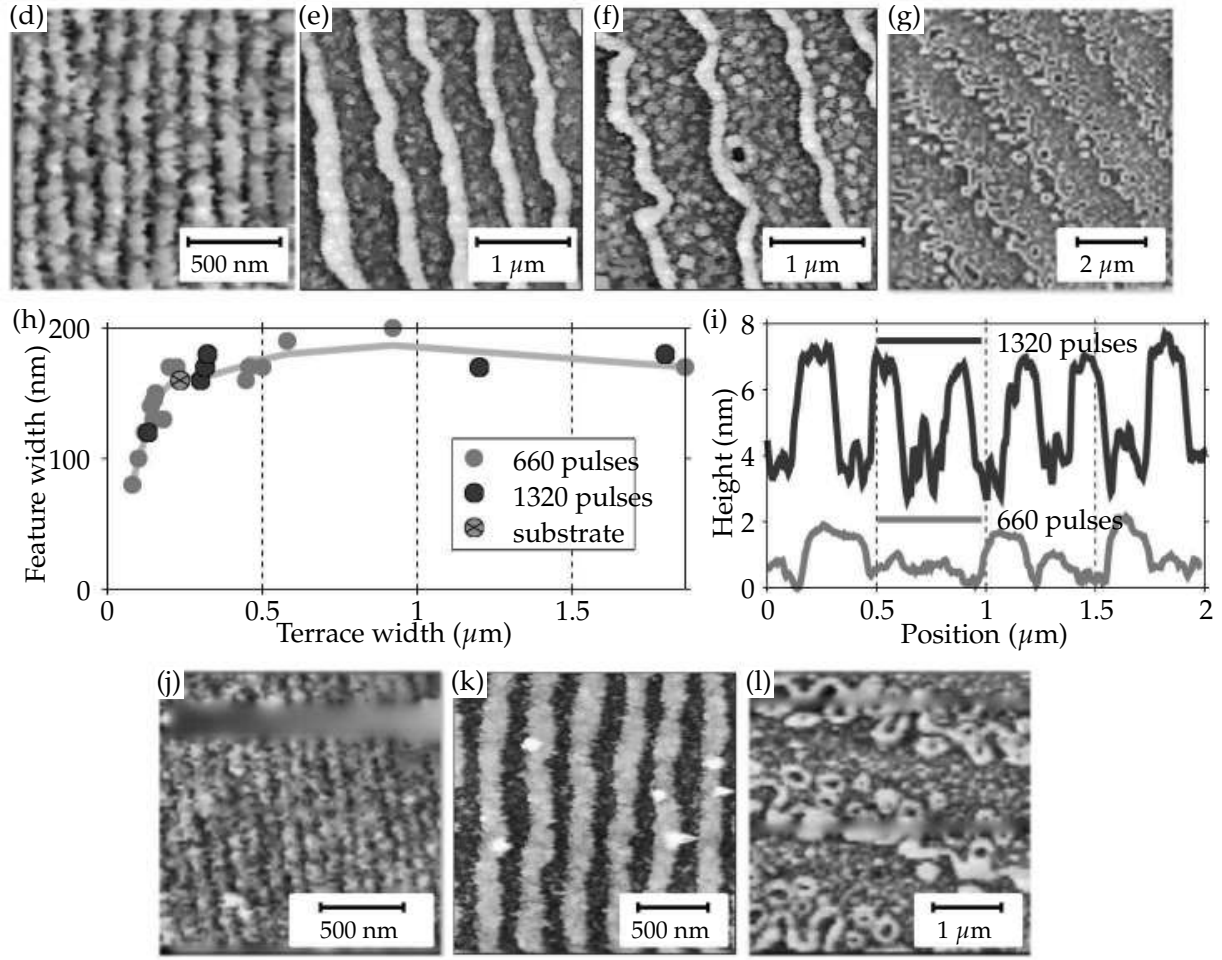

Figure 4.6: AFM height evolution of $\mathrm{SrRuO}_{3}$ on a $\mathrm{DyScO}_{3}$ substrate. (a) and (b) Micrographs of the substrate, height and phase. (c) Schematic drawing of the surface as polished with a bow shape (not to scale). (d), (e), (f) and (g) height images of $\mathrm{SrRuO}_{3}$ nanostructures after deposition of 660 pulses for various miscut angles and (j), (k) and (l) after 1320 pulses. (h) Feature width as a function of terrace width extracted from the AFM data, indicating the feature width saturates at a step width of $\sim 200 \mathrm{~nm}$. (i) Height profiles after deposition of 660 and 1320 pulses, indicating the feature height scales with the number of pulses.

ter deposition of 1320 pulses are shown in Fig. 4.6(j-1) and the step-width and feature-size is plotted in Fig. 4.6(h) in red.

In summary, a constant feature-size of roughly $170 \mathrm{~nm}$ was observed for all step-widths above $170 \mathrm{~nm}$. After the deposition of additional material on $\mathrm{SrRuO}_{3}$ patterns, the features only increased in height, not in width. The observed feature 

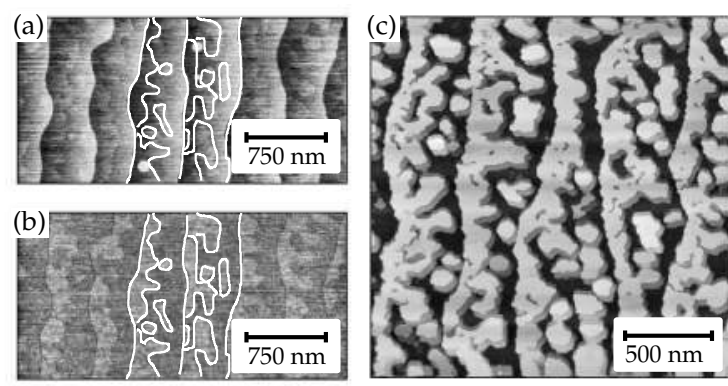

Figure 4.7: $\mathrm{NdGaO}_{3}$ (110) substrate height (a) and phase (b) TM-AFM images and the resulting $\mathrm{SrRuO}_{3}$ pattern after deposition (c). The white lines in (a) and (b) are drawn to clarify the edges of the mixed terminated regions.

sizes at a step-width of roughly $300 \mathrm{~nm}$ was equal to the mixed termination areas of the $\mathrm{DyScO}_{3}$ substrate.

\subsubsection{Termination selective growth other substrates}

Apart from $\mathrm{DyScO}_{3}$ (110) discussed above, also $\mathrm{SrTiO}_{3}$ (001) substrates can show ordered mixed termination after annealing. ${ }^{[58,74,76]}$ Moreover, $\mathrm{SrRuO}_{3}$ nanostructures are reported to be created on various substrates, such as, $\mathrm{LaAlO}_{3}{ }^{[75]}$ and LSAT ${ }^{[77]}$. In Fig. 4.7(a,b), a mixed terminated $\mathrm{NdGaO}_{3}$ substrate is depicted. Ordered areas offset in height and phase contrast were visible on the substrate terraces. The step edges were curved, as well as the edges of the differently terminated surfaces areas. The curved features were probably caused by the large step width of $\sim 400 \mathrm{~nm}$. After growth of $\mathrm{SrRuO}_{3}$ shown in Fig. 4.7(c), structures which mimicked the substrate termination pattern were observed. The observed mixed termination pattern of $\mathrm{NdGaO}_{3}$ and subsequent nanostructure formation was very similar to that of $\mathrm{SrRuO}_{3}$ on $\mathrm{DyScO}_{3}$.

\subsection{Structural and electronic characterization}

Below the structure, interface configuration (surface termination layer) and local electronic properties of nanowire patterns are reported, by using X-ray Diffraction, electron microscopy and STM.

\subsubsection{Structural characterization}

Structural analysis was performed of a patterned $\mathrm{SrRuO}_{3}$ film on a $\mathrm{DyScO}_{3}$ substrate. Reciprocal lattice maps of a sample showing $\mathrm{SrRuO}_{3}$ features of about $7 \mathrm{~nm}$ in height and $160 \mathrm{~nm}$ in width were recorded in order to determine if the $\mathrm{SrRuO}_{3}$ is grown epitaxially. Maps around the $(44 \overline{4}),(260),(444)$ and $(620)$ $\mathrm{DyScO}_{3}$ substrate peaks are shown in Fig. 4.8. The highest intensity peaks were 


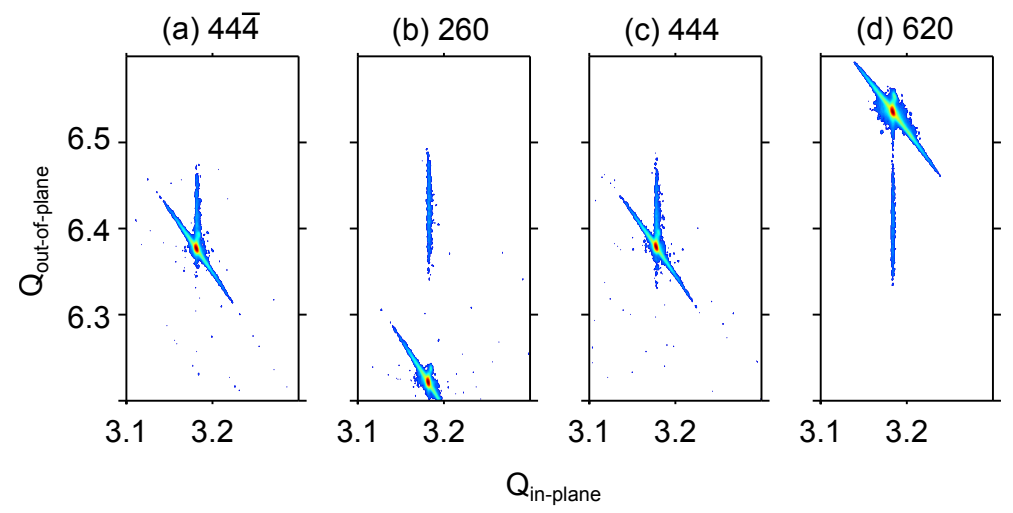

Figure 4.8: Reciprocal lattice maps of $\mathrm{SrRuO}_{3}$ on $\mathrm{DyScO}_{3}(110)$ around the $\phi=0^{\circ}(44 \overline{4}), \phi=$ $90^{\circ}(260), \phi=180^{\circ}$ (444) and $\phi=270^{\circ}$ (620) $\mathrm{DyScO}_{3}$ orthorhombic substrate peaks. Film peaks of lower intensity were found at similar $2 \theta$ values for all $\phi$ angles.

from the orthorhombic $\mathrm{DyScO}_{3}$ substrate and the lower intensity peaks were $\mathrm{SrRuO}_{3}$ films peaks. The $\mathrm{DyScO}_{3}$ showed a clear orthorhombic crystal structure, while the $\mathrm{SrRuO}_{3}$ films was nearly tetragonal. This is in good agreement with literature findings. ${ }^{[79]}$ Using the structural parameters reported by Vailionis et al. ${ }^{[79]}$ as input parameters, the structure was refined based on the data from Fig. 4.8 to a monoclinic structure $(\mathrm{a}=5.57 \AA, \mathrm{b}=5.57 \AA, \mathrm{c}=7.90 \AA$ and $\gamma=90.37^{\circ}$ ). The observed in-plane ordering and peak positions compared well with continues thin films, indicating patterned $\mathrm{SrRuO}_{3}$ samples using mixed terminated substrates are epitaxial and show a similar crystal structure as compared to conventional thin films. ${ }^{[79]}$

\subsubsection{Interface structure and configuration}

The structure as measured by $\mathrm{XRD}$ indicated the $\mathrm{SrRuO}_{3}$ patterns were epitaxially grown on $\mathrm{DyScO}_{3}$ (110) substrates. However, the exact atomic stacking at the interface is not known. It is especially interesting to confirm that $\mathrm{SrRuO}_{3}$ selectively grows on $\mathrm{ScO}_{2}$ terminated areas. Therefore, the exact structure and atomic configuration at the interface was studied using TEM, see also section 2.3.1.

For this purpose a patterned $\mathrm{SrRuO}_{3}$ samples was prepared with an additional ferroelectric $\mathrm{PbTiO}_{3}$ layer with a thickness of $\sim 30 \mathrm{~nm}$. This $\mathrm{PbTiO}_{3}$ layer was grown directly after the $\mathrm{SrRuO}_{3}$ nanowires, without cooling down the sample. ${ }^{7}$ The $\mathrm{PbTiO}_{3}$ layer allowed for studying the $\mathrm{DyScO}_{3}$ surface both near a $\mathrm{SrRuO}_{3}$ feature and in between $\mathrm{SrRuO}_{3}$ features, since the $\mathrm{PbTiO}_{3}$ grew epitaxially on both areas; the $\mathrm{DyScO}_{3}-\mathrm{PbTiO}_{3}$ interface can be studied in more detail than a possible $\mathrm{DyScO}_{3}$ - vacuum interface. The sample was prepared for TEM by focused ion beam milling, to create lamella perpendicular to the pseudo-cubic

\footnotetext{
${ }^{7}$ The morphology and growth of $\mathrm{PbTiO}_{3}$ on $\mathrm{SrRuO}_{3}$ nanowires was studied in detail by Smith et al. ${ }^{[80]}$, along with the effect of the electrode pattern on the ferroelectric properties of $\mathrm{PbTiO}_{3}$.
} 
[001] zone axis of $\mathrm{DyScO}_{3}$ and perpendicular to the nanowires.

Figure 4.9(a) shows a HAADF-STEM overview of the sample. $\mathrm{SrRuO}_{3}$ features were observed with a height of $\sim 5 \mathrm{~nm}$, a width of $\sim 120 \mathrm{~nm}$ and are spaced about $25 \mathrm{~nm}$ apart. Two-dimensional spectral images were acquired with STEMEELS to investigate the spatial distribution of the elements within the sample. Quantitative elemental maps are shown in Fig. 4.9(b), for Ru-M $\mathrm{M}_{4,5}$ (light-blue), $\mathrm{Sc}_{\mathrm{L}} \mathrm{L}_{2,3}$ (red) and $\mathrm{Ti}-\mathrm{L}_{2,3}$ (blue) edges. The distinct sample areas can clearly be identified as $\mathrm{PbTiO}_{3}$ (blue), $\mathrm{SrRuO}_{3}$ (light-blue), and $\mathrm{DyScO}_{3}$ (red).

In Fig. 4.9(c) a high resolution HAADF-STEM image of the sample is shown. Three zones are indicated: (1) interface between $\mathrm{DyScO}_{3}$ and $\mathrm{SrRuO}_{3}$; (2) interface between $\mathrm{SrRuO}_{3}$ and $\mathrm{PbTiO}_{3}$ and (3) interface between $\mathrm{DyScO}_{3}$ and $\mathrm{PbTiO}_{3}$. $\mathrm{Al}$ interfaces show coherent growth of both $\mathrm{SrRuO}_{3}$ and $\mathrm{PbTiO}_{3}$. To determine the exact stacking sequence two-dimensional STEM EELS spectral images of Dy, Sc, $\mathrm{Ru}$ and $\mathrm{Ti}$ are shown in Fig. 4.9(d,e). The corresponding line profiles are shown in Fig. 4.9(f). In region (1) the Sc signal of the $\mathrm{DyScO}_{3}$ substrate persists further outward compared to the Dy signal, while in region (3) the opposite is observed, indicating an stacking sequence in region (1) of $\mathrm{ScO}_{2}-\mathrm{SrO}$ and in region (3) of DyO- $\mathrm{TiO}_{2}$. The topmost surface of $\mathrm{SrRuO}_{3}$, in region (2) shows an interesting stacking, where an additional $\mathrm{SrTiO}_{3}$ layer seems to be formed at the interface between $\mathrm{SrRuO}_{3}$ and $\mathrm{PbTiO}_{3}$. This formation of $\mathrm{SrTiO}_{3}$ is discussed in more detail by Egoavil et al. ${ }^{[30]}$ and Smith et al. [80]

Smearing of intensity contrast near the edges of the $\mathrm{SrRuO}_{3}$ wires was observed near all nanowires at the vertical interface between $\mathrm{SrRuO}_{3}$ and $\mathrm{PbTiO}_{3}$. From the TEM data, the edges of the wires appear not to be straight, but rather curved. However, the TEM data showed an average of several $\mathrm{SrRuO}_{3}$ layers, due to transmission through several nanometers of material. The nanowires were meandering slightly, possibly resulting in the blurred edges in the TEM images.

Overall, the TEM results showed that the surface terminating layer of $\mathrm{DyScO}_{3}$ is $\mathrm{ScO}_{2}$ underneath the $\mathrm{SrRuO}_{3}$ nanowires and $\mathrm{DyO}$ in between the nanowires. Moreover, no $\mathrm{Ru}$ was found near the interface between the $\mathrm{DyScO}_{3}$ substrate and $\mathrm{PbTiO}_{3}$ film in between the $\mathrm{SrRuO}_{3}$ wires, indicating $\mathrm{SrRuO}_{3}$ only nucleated on the $\mathrm{ScO}_{2}$ termination. The interfaces between $\mathrm{SrRuO}_{3}$ and $\mathrm{DyScO}_{3}$ and $\mathrm{PbTiO}_{3}$ and $\mathrm{DyScO}_{3}$ were found to be atomically sharp and well-defined.

\subsubsection{Local electronic properties}

Experimental tunneling AFM (TUNA) results recorded on a wire patterned sample $^{8}$ are shown in Fig. 4.10. The AFM height image, Fig. 4.10(a), and TUNA current map, Fig. 4.10(b), showed the same pattern. Each $\mathrm{SrRuO}_{3}$ wire had a finite tunneling current, while the uncovered areas did not show any transport, as can be seen in the line profiles plotted in Fig. 4.10(c). Most wires had a different tunneling current. For example the wire observed around $x=0.4 \mu \mathrm{m}$ had a TUNA signal which was an order of magnitude smaller compared to most other wires.

\footnotetext{
${ }^{8}$ TUNA performed on the same sample as shown in Fig. 4.4(b).
} 

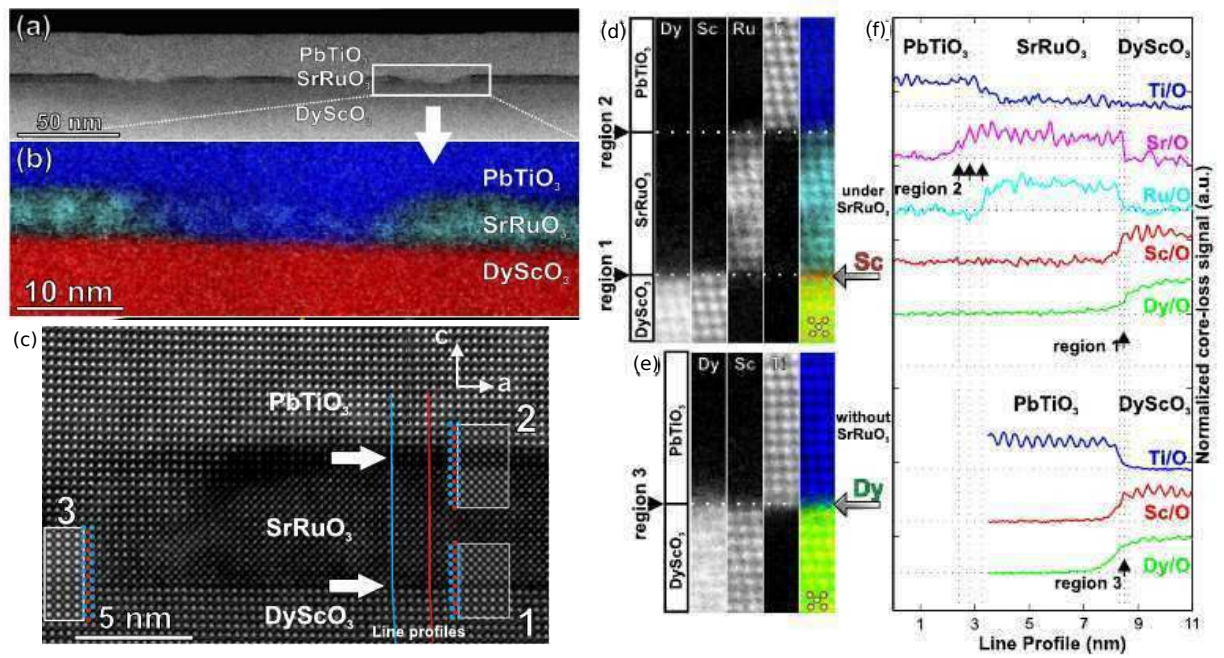

Figure 4.9: TEM images of a $\mathrm{SrRuO}_{3}$ wire pattern covered with $30 \mathrm{~nm}$ of $\mathrm{PbTiO}_{3}$ on a $\mathrm{DyScO}_{3}$ substrate. In (a) an HAADF-STEM overview is shown and in (b) two-dimensional STEM-EELS quantitative elemental maps for Sc (red), Ti (blue) and Ru (light blue) are plotted. In (c) a high resolution image is shown where three zones are indicated, which are studied using STEM-EELS in (d) and (e) as maps and in (f) as line profiles in order to study the interface configurations. These elemental maps indicate a stacking sequence of $\mathrm{ScO}_{2}-\mathrm{SrO}$ near a $\mathrm{SrRuO}_{3}$ wire and $\mathrm{DyO}-\mathrm{TiO}_{2}$ in between the wires. Moreover, no $\mathrm{SrRuO}_{3}$ was found at the $\mathrm{DyScO}_{3}-\mathrm{PbTiO}_{3}$ interface, indicating $\mathrm{SrRuO}_{3}$ only nucleated on the $\mathrm{ScO}_{2}$ terminated areas.

Upon changing the sample bias, no Ohmic behavior was observed. Therefore, no quantitative statements can be made about the conductivity of the wires using this configuration. However, since each wire showed a distinctly different conductivity, they were found to be electrically insulated from each-other over a long range.

\subsection{Discussion}

\section{Nanowire PLD growth}

$\mathrm{DyScO}_{3}$ substrates showing an ordered striped phase of different chemical surface terminations can be selected based on AFM morphology and phase or friction contrast. By varying the anneal time, the morphology can be tuned as is observed in Fig. 4.2 for $\mathrm{DyScO}_{3}$ substrates annealed for 30 minutes and 4 hours. Subsequent growth of $\mathrm{SrRuO}_{3}$ using PLD results in nanostructures, which closely resembled the mixed termination pattern of the $\mathrm{DyScO}_{3}$ substrate, as shown in Fig. 4.4.

The observed RHEED patterns and time evolution of the specular spot during 


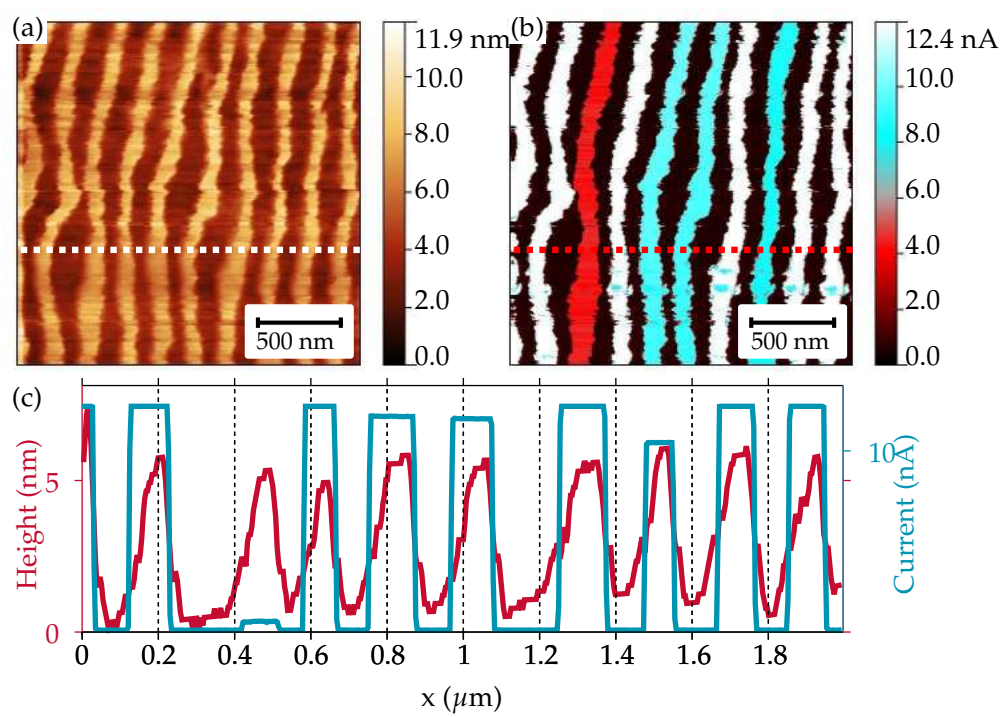

Figure 4.10: TUNA conducting AFM images on a $\mathrm{SrRuO}_{3}$ nanowire patterned $5 \times 5 \mathrm{~mm}^{2}$ sample. Sample edges were grounded. In (a) the AFM height is shown, (b) TUNA current recorded at $100 \mathrm{mV}$ sample bias, (c) line profile of height and TUNA current (dotted lines in $(a, b)$ indicate the location of the line profile).

deposition for the growth of $\mathrm{SrRuO}_{3}$ nanostructures is comparable to the growth of $\mathrm{SrRuO}_{3}$ on $\mathrm{SrTiO}_{3} \cdot{ }^{[71]}$ Diffraction patterns of $\mathrm{SrRuO}_{3}$ nanostructures are dominated by spots which are aligned on a Laue circles. These patterns indicate an atomically smooth crystalline top surface, in accordance with the AFM analysis of the nanowire top surface structures. The small initial oscillation, observed after $\sim 20$ seconds, is not well-understood. This small oscillation is not observed for growth on singly terminated (chemically treated) $\mathrm{DyScO}_{3}$. Therefore, it is likely related to surface morphology of the mixed terminated $\mathrm{DyScO}_{3}$ substrate. A possible explanation is that the $\mathrm{ScO}_{2}$ terminated area, which the $\mathrm{SrRuO}_{3}$ selectively nucleates on, has some initial surface roughness at an atomic scale, as for example observed in Fig. 3.3(a). $\mathrm{SrRuO}_{3}$ might first resolve this atomic roughness during the initial phase of the growth, giving rise to a small increase in the RHEED intensity.

\section{Nanowire properties and structure}

The interface between $\mathrm{DyScO}_{3}$ and $\mathrm{SrRuO}_{3}$, studied by TEM, is shown to be atomically sharp. Using STEM EELS, the terminating layer of the $\mathrm{DyScO}_{3}$ substrate is shown to be $\mathrm{ScO}_{2}$ where $\mathrm{SrRuO}_{3}$ is grown and $\mathrm{DyO}$ in between the $\mathrm{SrRuO}_{3}$ features, indicating that the patterned growth of $\mathrm{SrRuO}_{3}$ is directly related to the surface termination, as expected from phase contrast and frictionforce AFM studies of the $\mathrm{DyScO}_{3}$ substrate.

Conductive AFM (TUNA) experiments show a large contrast in electrical prop- 
erties on and off the $\mathrm{SrRuO}_{3}$ wire features. Wires are found to be conductive and well-isolated from each other over long distances. Since no Ohmic behavior was observed no quantitative statements can be made. However, this large electrical contrast of nanopatterned electrodes provides open new routes for analysis of, i.e., ferroelectric nanostructures. ${ }^{[80]}$

$\mathrm{SrRuO}_{3}$ nanostructures width a height of $\sim 7 \mathrm{~nm}$, show a crystal structure, which is comparable to that of a continuous thin films, as measured using reciprocal lattice maps (XRD). The nanostructured films are coherently grown on $\mathrm{DyScO}_{3}$ (110) substrates (cube-on-cube type). The structure of $\mathrm{SrRuO}_{3}$ on $\mathrm{DyScO}_{3}$ is nearly tetragonal with a small monoclinic distortion. $\mathrm{SrRuO}_{3}$ nanowires grown on mixed terminated $\mathrm{SrTiO}_{3}$ substrates are found the be twinned ${ }^{9}$ by Kan and Shimakawa ${ }^{[81]}$ and show a different temperature dependence compared to continuous films. Such twinning is not observed in this work on $\mathrm{SrRuO}_{3}$ structures on $\mathrm{DyScO}_{3}$ substrates.

\section{Nanowire growth model}

The resulting $\mathrm{SrRuO}_{3}$ nanowires and islands have shapes and dimensions similar to the substrate surface mixed termination pattern. In Fig. 4.4(a) a clear example is found, where both dots and lines of $\mathrm{SrRuO}_{3}$ form, mimicking the substrate termination pattern, shown in Fig. 4.2(a). When changing the miscut angle (terracewidth) on a single substrate, no effect was found on the average feature size. On areas with a terrace width of $170 \mathrm{~nm}$ or above, a typical feature size of $\sim 170 \mathrm{~nm}$ was found, independent of the miscut angle, as shown in Fig. 4.6.

On low miscut substrate areas, islands are formed in between the $\mathrm{SrRuO}_{3}$ nanowires, similar to islands observed in Fig. 4.2(a). Since both the annealing of the $\mathrm{DyScO}_{3}$ substrate and the growth of $\mathrm{SrRuO}_{3}$ are diffusive processes, both could yield islands if the terrace width is larger than the diffusion length. However, since the feature size of the islands is of similar length as the mixed terminated areas of the substrate, it seems reasonable to assume the size and distribution of islands is induced by the $\mathrm{DyScO}_{3}$ substrate. This implies that the typical feature size of $170 \mathrm{~nm}$ is predominantly caused by diffusion processes which occurred during the annealing of the substrate. The spacing of the $\mathrm{SrRuO}_{3}$ features is related to the diffusivity on $\mathrm{DyO}$ during $\mathrm{SrRuO}_{3}$ growth. However, small Bsite nucleation points could be present on the DyO terminated areas, giving rise to island formation. Therefore, it is difficult to quantify the diffusion length of $\mathrm{SrRuO}_{3}$ on DyO. Results shown in Fig. 4.4(c), indicate the diffusion length to be at least $50 \mathrm{~nm}$. Deposition of additional $\mathrm{SrRuO}_{3}$ layers, up to $\sim 4 \mathrm{~nm}$ in height, does not affect the average feature-width.

In literature, similar growth phenomena are reported on $\mathrm{SrTiO}_{3} \cdot{ }^{[58,74]}$ Initially, this type of ordered $\mathrm{SrRuO}_{3}$ growth was attributed to the surface step edges. While latter, it was attributed to a difference in adatom sticking coefficient on different surface termination. In the work presented in this chapter, no relationship

\footnotetext{
${ }^{9}$ Twinning of $\mathrm{SrRuO}_{3}$ on $\mathrm{SrTiO}_{3}$ occurs not only for nanowire structures, but also in continuous films, due to pre-existing defects in $\mathrm{SrTiO}_{3}$ substrates.
} 
with the step edges was found. Apart from the fact that the surface mixed termination on the $\mathrm{DyScO}_{3}$ substrate orders along the vicinal steps. Moreover, it was found that all material sticks to the surface and no evaporation takes place.

The amount of material deposited using PLD at the given conditions is the same as the volume of the features formed during growth. All the material thus sticks and diffuses to form the nanowire and islands structures. This implies a surface diffusion process occurs during growth where adatoms diffuse along the surface. In the special case of $\mathrm{SrRuO}_{3}$ previous studies have shown growth of $\mathrm{SrRuO}_{3}$ on different surface terminations shows different surface diffusivity. ${ }^{[71]}$ When the surface diffusion on $\mathrm{DyO}$ is high compared to $\mathrm{ScO}_{2}$ and $\mathrm{SrRuO}_{3}$ itself material will nucleate preferentially onto the $\mathrm{ScO}_{2}$ and $\mathrm{SrRuO}_{3}$ terminated areas. This can be explained, since the adatom density, $\eta$, scales linearly with the flux F and scales inversely with the diffusivity, D: $\eta \sim \frac{F}{D} \cdot{ }^{[82]}$ A local difference in diffusivity will thus yield a local difference in nucleation probability, resulting in preferential growth. The kinetics of this growth mode can be simulated using a Solid-on-Solid type Monte Carlo model. This is done in chapter 5.

\subsection{Conclusions}

In this chapter, coherently grown $\mathrm{SrRuO}_{3}$ nanowires on doubly terminated templates are obtained by means of self-organization on the mixed terminated $\mathrm{DyScO}_{3}$ and $\mathrm{NdGaO}_{3}$ surfaces. This type of self-organization is the result of a surface diffusion process, since the volume of the nanostructures is equal to the volume of a continuous thin film sheet deposited at the same conditions; all the material sticks and diffuses. During pulsed laser deposition $\mathrm{SrRuO}_{3}$ mimics the surface mixed termination template, independent of terrace width. Effectively, the substrate mixed termination pattern acts as a template for the growth on conducting $\mathrm{SrRuO}_{3}$ nanostructures for thin films. The nanowire structures are typically between 100 and $200 \mathrm{~nm}$ in width and made up to $8 \mathrm{~nm}$ in height. The length of these nanowires is found to be at least $25 \mu \mathrm{m}$, but is likely coupled to the terrace step structure, which can have step-lengths comparable to the sample size of $5 \mathrm{~mm}$. The $\mathrm{SrRuO}_{3}$ structures are epitaxially grown and have a nearly tetragonal structure on $\mathrm{DyScO}_{3}$ (110) substrates, The formed interfaces are atomically sharp and are found to have a stacking sequence of $\mathrm{ScO}_{2}-\mathrm{SrO}$ underneath a nanowire. While a DyO terminating layer is found in between the $\mathrm{SrRuO}_{3}$ wires. Indicating $\mathrm{SrRuO}_{3}$ nucleates selectively on the $\mathrm{ScO}_{2}$ surface terminated areas of $\mathrm{DyScO}_{3}$ (110). These highly ordered conducting nanostructures can be used to study the physical properties of complex oxides with reduced dimensions or act as a starting point to create laterally controlled heterostructures. In the latter case, their in-plane interaction could lead to a vast range of unexplored physical properties. 


\title{
Chapter 5
}

\section{Growth kinetics on termination templates}

\begin{abstract}
Commonly used conducting metal-oxide $\mathrm{SrRuO}_{3}$ shows a fascinating form of self-organization during growth by pulsed laser deposition on perovskite substrates with a mixed surface termination. The atomistic nature of this type of growth is not well understood. Therefore, a threedimensional lattice kinetic Monte Carlo model is developed to study the time evolution of $\mathrm{SrRuO}_{3}$ growth on various termination templates. The templates are simulated by locally varying the activation barrier for diffusion, i.e., changing the local diffusivity. Relative energy barrier changes of $\sim 0.3 \mathrm{eV}$ between $\mathrm{A}$ and B-site surfaces are shown to give rise to self-organized nanopattern formation. A critical nanowire height behavior is found, which determines the time evolution of nanowire growth.
\end{abstract}




\subsection{Introduction}

Growth of epitaxial thin films is of great interest for creating functional heterostructures. Much effort is typically directed towards achieving smooth surfaces and interfaces. A layer-by-layer type growth mode is often desired, as it yields atomically smooth layers. However, this growth mode is not always readily obtained, as it depends on both thermodynamics and growth kinetics. In pulsed laser deposition, the high supersaturation helps to achieve a high nucleation density, which can promote layer-by-layer growth.

In an interesting case of $\mathrm{SrRuO}_{3}$ heteroepitaxy, initial layer-by-layer growth is observed on single $\mathrm{TiO}_{2}$ terminated $\mathrm{SrTiO}_{3}$ (001) substrates. However, after completion of the initial monolayer, a growth mode transition occurs towards a stepflow like growth mode. ${ }^{[83,84]}$ This growth mode transition coincides with a termination conversion from $\mathrm{TiO}_{2}$ to $\mathrm{SrO}$. ${ }^{[71]}$ The termination conversion is attributed to the volatility of $\mathrm{RuO}_{x}$ species. Moreover, growth of $\mathrm{SrRuO}_{3}$ is shown to be highly sensitive to the local surface termination of the substrate ${ }^{[57,58]}$. For example, self-organized (nanowire) growth on mixed $\mathrm{DyO} / \mathrm{ScO}_{2}$ terminated $\mathrm{DyScO}$ (110) substrates is observed, as mentioned in chapter 4. For step-flow like growth and self-organization to occur, a sufficiently high surface diffusivity is required, depending on the stepdensity of the substrate. $\mathrm{SrRuO}_{3}$ clearly shows this high diffusivity, as under certain conditions also an extreme case of step-flow growth can occur: step-bunching. ${ }^{85,86]}$ Self-assembled growth is expected to occur when there is a local variation in the surface diffusivity. For example a high diffusivity on A-site and a lower diffusivity on B-site.

In order to model the interesting behavior of $\mathrm{SrRuO}_{3}$ growth during PLD, a lattice kinetic Monte Carlo (kMC) model is employed. Atomistic simulations using kMC models are well-suited to study kinetic effects, coarsening and coalescence at physically relevant time scales for film growth. ${ }^{[4,87,88]} \mathrm{kMC}$ type models have been used extensively in order to study growth of metal/semiconductor systems, as well as several oxide systems. ${ }^{[89-98]}$ Moreover, kMC models are previously used to explain atomistic growth during oxide homoepitaxy, e.g., layerby-layer and step-flow growth. ${ }^{[99,100]}$. In such models, the growth of complex oxides, like $\mathrm{SrRuO}_{3}$, is simplified by assuming deposition of complete pseudo-cubic unit-cell blocks and all processes are modeled using Arrhenius type interactions.

For simulating thin film growth, often $\mathrm{kMC}$ models with a two-dimensional lattice or grid are used. For thin film growth this approximation can be validated due to the two-dimensional nature of thin films. However, in order to simulate diffusion, nucleation and growth of nanowires, a model with a three-dimensional lattice was developed, which is well-suited to simulate vertical growth of nanostructures and islands.

In this chapter, the growth of $\mathrm{SrRuO}_{3}$ on mixed terminated templates is simulated using a three-dimensional $\mathrm{kMC}$ model. The activation barrier for diffusion is varied locally, in order to simulate local difference in surface termination; either A or B-site. A kMC model is used to study the time-evolution of self-organized 
growth of $\mathrm{SrRuO}_{3}$ nanowires. The wire patterns observed in chapter 4 , as well as the continues film growth observed in chapter 3 are simulated. First, the effect of this local difference in activation barrier is studied, in order to confirm the validity of the model proposed in chapter 4 . The energy barrier difference between A and B-site terminated areas should be physically realistic. Secondly, the timeevolution of the self-organized nanowire growth is simulated in detail and modeled using a critical nanowire height behavior. Finally, the kMC model is used to study and predict $\mathrm{SrRuO}_{3}$ growth behavior on perfect singly A-site terminated templates, which are not readily available in experiments. Prior to discussing the simulation results, the commonly observed types of thermodynamic and kinetic growth are discussed. Followed by the details of the three-dimensional kMC model.

\subsubsection{Atomistic view on thin film growth}

Epitaxial growth can occur in various growth modes, resulting in different surface morphologies. The type of growth-mode depends on the thermodynamic properties of the materials involved and on the amount of misfit strain. Thermodynamics is suitable to describe growth when the system is near equilibrium, giving rise to three types of growth modes. These models can be classified based on the relative surface energies of the substrate and film. However, if the diffusion time and length are limited, kinetic effects start to play a role, resulting in additional growth modes. In this context it is common to refer to a system being in the thermodynamic limit when the time scales are large and the system is allowed to minimize its surface energy. Alternatively, the system is said to be in the kinetic limit when short time-scale interactions dominate and the system might get fixed in a local minimum. The pulsed nature of PLD and the high supersaturation often result in high nucleation rates and short time scale (kinetic) interactions dominate growth. Below, the three common thermodynamic growth modes, as well as two types of kinetic growth modes, are discussed.

\section{Growth in thermodynamic equilibrium}

The three growth modes to describe crystal growth are layer-by-layer growth ${ }^{[101]}$, island growth and Stranski-Krastanov growth. ${ }^{[102]}$ Based on the interfacial tension or surface energy of the film $\left(\gamma_{F}\right)$, substrate $\left(\gamma_{S}\right)$ and substrate-film interface $\left(\gamma_{I}\right)$, island growth and layer growth can be explained. Layer-by-layer or Frank-van der Merwe growth, occurs when the film material wets the surface $\left(\gamma_{S} \geq \gamma_{F}+\gamma_{I}\right)$ and nucleation occurs predominantly in the two in-plane directions. Island growth or Volmer-Weber growth, occurs when the bonding between atoms is stronger in the film than between the film and substrate. Therefore, the film material does not wet the surface $\left(\gamma_{S}<\gamma_{F}+\gamma_{I}\right)$. In heteroepitaxial growth, an intermediate growth mode can occur, called Stranski-Krastanov growth, where initially the film wets the surface. However, after several monolayers, islands growth occurs due to the applied strain. 


\section{Kinetics of crystal growth}

When the system is not in thermodynamic equilibrium, kinetic effects start to play a role in determining the resulting morphology. High supersaturation during pulsed deposition will result in a high nucleation density, which limits the diffusion of adatoms on the surface. The time particles are allowed to diffuse, before a new pulse is applied, is limited. Depending on the diffusion length and nucleation density, this can give rise to different kinetically limited growth modes.

If the diffusion length on terrace stepped surfaces, $l_{D}$, is larger than the terrace width, $l_{t}$ : adatoms can diffuse towards the terrace step edge and attach to it. In this high-diffusivity growth mode or step-flow growth, deposited adatoms nucleate at the pre-existing terrace steps, while nucleation on the terraces is limited; intralayer mass transport is high. This results in an effective motion or flow of the terrace step edges. In an extreme case of step-flow growth, terrace steps can merge and form multiple unit-cell high vertical steps. For example, when no barrier is present for adatoms to cross a step edge (interlayer transport), larger terrace can move faster compared to smaller terraces, resulting in step-bunching. ${ }^{[103]}$

When the intralayer mass transport is limited, nucleation on the terrace will occur. Initially, small nuclei will form, which will grow latterly to form islands. Depending on the amount of interlayer mass transport, two growth regimes can be classified. If interlayer mass transport is limited, second layer nucleation will occur on-top of the initial islands, resulting in multilayer growth. However, if a steady interlayer mass transport is present, second layer nucleation can be prevented and layer-by-layer growth occurs. A critical island radius, $R_{C}$ can be defined, which determines the mean island radius at which second-layer nucleation starts to occur. The critical radius depends on the amount of interlayer mass transport ${ }^{1}$, the mean island spacing and the material flux, as described in detail by Tersoff et al. ${ }^{[82]}$.

The diffusion length, $l_{D}$, scales with the diffusion coefficient $D_{S}$ and the $\tau$ the residence time before re-evaporation: $l_{D} \approx \sqrt{D_{S} \tau}$. The diffusion coefficient is defined as in Eq. (5.1). ${ }^{[99]}$

$$
D_{S}=v \alpha^{2} e^{\left(\frac{-E_{A}}{k_{B} T}\right)}
$$

Here, $\alpha$ is equal to the characteristic jump distance, $v$ is the attempt frequency for diffusion, typically close the Debye frequency $\sim 10^{13}$ and $\mathrm{E}_{\mathrm{A}}$ is the activation energy for diffusion.

\subsection{Monte Carlo type growth simulations}

The kinetic effects of the mentioned growth modes, can be described by kinetic Monte Carlo (kMC) type models. In such models the diffusion of adatoms is

\footnotetext{
${ }^{1}$ The critical island size, $R_{C}$, is strongly influenced by the energy barrier adatoms have to overcome in order to descend across a step edge to a lower terrace. If this barrier is high, second layer nucleation is enhanced and $R_{C}$ is reduced.
} 
simulated by calculating the hopping rates $(\mathrm{k})$ for all atoms on a simulated surface grid or lattice. The hopping rates are calculated using Eq. (5.2), where $E_{D}=$ $E_{S}+n E_{N}$. $E_{S}$ is the substrate contribution and $E_{N}$ scales with the coordination number, $\mathrm{n}$. One atom is selected to diffuse based on its hopping rate. The hopping event is performed, the hopping rates updated and another event is selected. This process is continued until the desired amount of simulation time has elapsed. Details on the algorithm are given in section 2.4.3.

$$
k=k_{0} \cdot e^{\left(\frac{-E_{\mathrm{D}}}{k_{\mathrm{B}} T}\right)}
$$

A two-dimensional Solid-on-Solid (SOS) ${ }^{[46,87,88]}$ type model is used as a starting point for the $\mathrm{kMC}$ model used in this work. Perfect sticking is assumed, i.e., no re-evaporation is considered.

The two-dimensional nature of the simulation grid in SOS-type simulations requires additional definition of diffusion across step edges, to tune interlayer mass transport. When an adatom moves to another step terrace, it has to cross the step edge. Typically, an additional energy barrier is introduced for step-up and step-down motion, called the Ehrlich-Schwoebel ${ }^{[104-106]}$ barrier, $E_{e s}$. This barrier is added to the activation energy for adatoms near a step edge and can be asymmetric with regard to the direction of the movement. A positive barrier will reduce interlayer mass transport. However, negative $E_{e s}$ barriers are also reported in literature. ${ }^{[107]}$ In case of negative $E_{e s}$ barriers, interlayer mass transport is enhanced, which will reduce second-layer nucleation and promote layer-bylayer or step-flow growth.

\subsubsection{Three-dimensional kinetic Monte Carlo model}

The Solid-on-Solid type kMC model, which is often used for simulating thin film growth, uses a two-dimensional grid or lattice. Adatoms are only allowed to move in-plane. This can be validated in the case of thin film growth, due to the two-dimensional nature of thin films. However, during $\mathrm{SrRuO}_{3}$ nanowire growth, vertical features of up to 20 monolayer in height are formed. An intuitive way to simulate vertical diffusion of adatoms, is to allow adatoms to diffuse upwards along vertical steps. This requires the addition of a third dimension, compared to conventional SOS-type models. A three-dimensional model allows for adatoms to diffuse across the surface and along the sides of vertical features, in a similar fashion. Note, that gravity has no effect at these length scales and diffusion along a vertical and horizontal lattice is physically equivalent.

In this work the SOS type kMC model is extended for the use of three-dimensional grids or lattices. The number of nearest neighbors is increased to six in three dimensions. However, to allow for interlayer adatom transport, also diagonal hopping is allowed, increasing the total neighbor count to 18. A schematic of this three-dimensional $\mathrm{kMC}$ model and the considered neighbors is given in Fig. 5.1(a), where several adatoms are plotted (dark gray) on a three-dimensional grid (light gray) and their allowed hopping direction are indicated (gray). The 


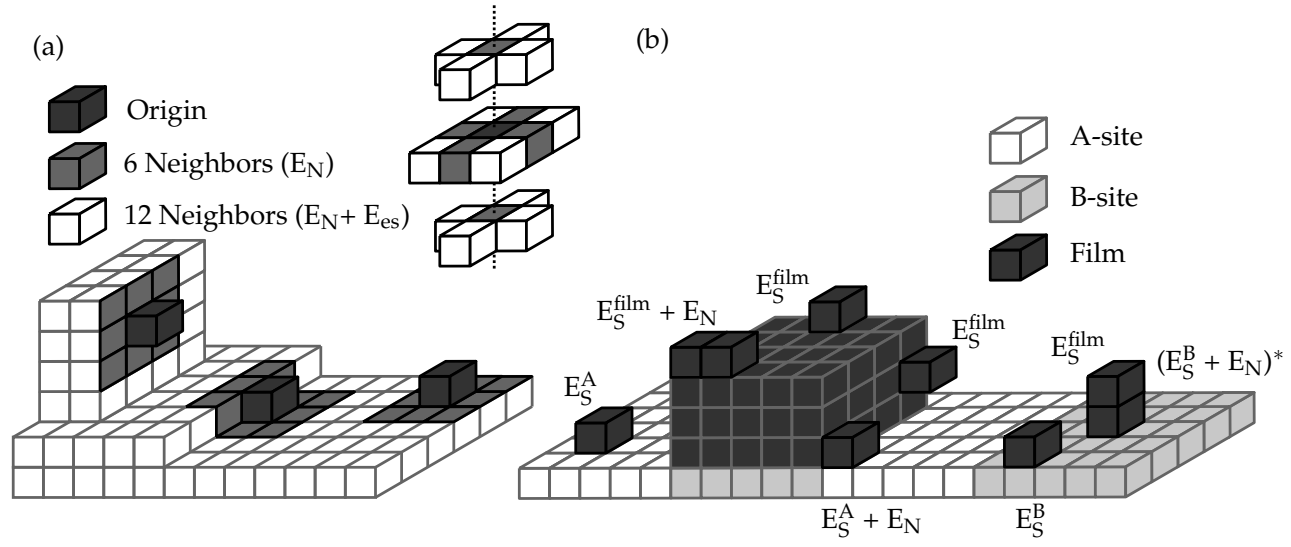

Figure 5.1: Schematics of the three-dimensional kMC model. (a) An overview of the possible movement direction of atoms depicted in a dark color. These atoms are allowed to move to the surrounding shaded positions. All of the 18 considered movement directions are indicated above the terrace structure. (b) Examples of energy barriers in this kMC model. All film atoms are labeled with their corresponding diffusion barrier. The atom marked $(*)$ has its hopping rate set to zero in order to prevent the atom on top of it from starting to float.

energy barriers for diffusion, $E_{D^{\prime}}^{i}$ are calculated using Eq. (5.3), for hopping in direction i.

$$
E_{D}^{i}=E_{S}^{A, B, f i l m}+n E_{N}+\delta_{i} E_{e s}
$$

Here, $\mathrm{E}_{\mathrm{S}}$ and $\mathrm{E}_{\mathrm{N}}$ are similar to a SOS-type model. For the hopping rate calculation only the six nearest neighbor are taken into account, in order to acquire similar hopping rates as are obtained in the SOS model and in the literature. While, for determining the hopping direction, all 18 neighbors are considered. To control the amount interlayer adatom transport, an $\mathrm{E}_{\mathrm{es}}$ barrier is applied for hops in the 12 diagonal directions. Therefore, $\delta_{i}=0$ for $i=1 \ldots 6$ and $\delta_{i}=1$ for $i=7, \ldots, 18$, where $i \leq 6$ represents hopping in one of the six cubic directions.

Areas of different surface termination are modeled by adjusting the static (substrate) contribution, $\mathrm{E}_{\mathrm{S}}$, to the diffusion barrier. Compared to the homoepitaxial diffusion barrier, $E_{S}^{\text {film }}$, a higher energy barrier for diffusion is set on B-site terminated surface areas. A lower barrier is set on A-site areas $\left(\mathrm{E}_{\mathrm{S}}^{\mathrm{A}}\right)$ compared to the barrier on B-site areas, $\left(\mathrm{E}_{\mathrm{S}}^{\mathrm{B}}\right)$.

An overview of the parameters used is given in Table 5.1. The parameters are selected based on experimentally obtained values for the activation energy measured using RHEED ${ }^{[99,108]}\left(\mathrm{E}_{\mathrm{A}}=0.5-2.2 \mathrm{eV}\right)$ and literature values used for $\mathrm{E}_{\mathrm{N}}$ in oxides ${ }^{[90]}\left(\mathrm{E}_{\mathrm{N}}=0.25-0.6 \mathrm{eV}\right)$. These values are also comparable to parameters used to simulate the growth of semiconductors on silicon using molecular beam epitaxy. ${ }^{[109]}$ 
Table 5.1: Three-dimensional kMC settings. Parameters based on the combined work of Rijnders ${ }^{[99]}$, Koster ${ }^{[108]}$, Vvedensky et al. ${ }^{[109]}$ and Lam et al. ${ }^{[90]}$. The simulations are performed with a pulse repetition rate of $1 \mathrm{~Hz}$ with $k_{0}$ set to $10^{12} \mathrm{~Hz}$, equivalent to a repetition rate of $10 \mathrm{~Hz}$ at $k_{0}=10^{13} \mathrm{~Hz}$. The number of pulses required for the completion of a single monolayer was set to 30 . A grid of $256 \times 32$ u.c. $^{2}$ was used for most simulations.

\begin{tabular}{cccccc}
\hline \hline $\mathrm{E}_{\mathrm{S}}^{\text {film }}(\mathrm{eV})$ & $\mathrm{E}_{\mathrm{S}}^{\mathrm{A}}(\mathrm{eV})$ & $\mathrm{E}_{\mathrm{S}}^{\mathrm{B}}(\mathrm{eV})$ & $\mathrm{E}_{\mathrm{N}}(\mathrm{eV})$ & $\mathrm{E}_{\mathrm{es}}(\mathrm{eV})$ & $\mathrm{T}$ \\
\hline 1.1 & {$[0.9-1.0]$} & 1.3 & 0.25 & {$[-0.15-0.15]$} & $600^{\circ} \mathrm{C}$ \\
\hline \hline
\end{tabular}

In Fig. 5.1(b) several examples of the energy barriers for diffusion used for calculating the hopping rates are shown $\left(\delta_{i}=0\right)$. The energy barrier for diffusion of an adatom on A-site surface areas, $E_{S}^{A}$, is calculated by using a fixed $E_{S}$ value and an interaction term for the A-site substrate: $E_{D}=\left(E_{S}+E_{N}^{A}\right)=E_{S}^{A}$. The energy barrier for diffusion for an adatom on an A-site area, which has one neighbor: $E_{D}$ $=\left(E_{S}+E_{N}^{A}\right)+E_{N}^{\text {film }}=E_{S}^{A}+E_{N}$. One neighbor term, determines the effective value of $E_{S}$. The remaining five neighbors determine the contribution strength of $E_{N}$. In the discussion of the simulation results and parameters overview, $E_{\mathrm{N}}^{\mathrm{A}, \mathrm{B}}$, film

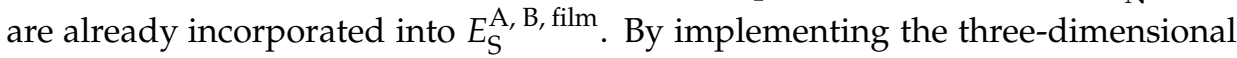
model in this way, the calculated hopping rates are similar to hopping rates in the two-dimensional model used in literature.

Note that, this three-dimensional model allows for overhang of adatoms. This overhang facilitates the transport of adatoms along vertical steps. However, prior to every material pulse the amount of overhang still present is limited, as typically large islands are formed and little no second layer nucleation occurs. ${ }^{2}$

\subsection{Growth simulations on termination templates}

The effects of substrate mixed termination patterns on the time evolution of the growth of $\mathrm{SrRuO}_{3}$ nanowires is studied in this section. The result of an adapted three-dimensional SOS-type model are discussed, which is able to simulate true vertical growth of nanowires and islands. The $\mathrm{kMC}$ model is first tested on a simple starting template, a singly terminated substrate. Next, the model is used to calculate the growth evolution of nanowires on ordered mixed terminated templates. The time evolution of the wire growth is studied quantitatively and modeled. The effect of $\mathrm{E}_{\mathrm{S}}^{\mathrm{A}}$ on the wire growth is studied. Finally the model is used to predict the growth of $\mathrm{SrRuO}_{3}$ on singly A-site terminated surfaces, which are not readily obtained experimentally.

\footnotetext{
${ }^{2}$ In equilibrium, the volume attributed to overhang situations in the kMC simulations is below 10 u.c. $^{2}$ on a $256 \times 32$ u.c. $^{2}$ lattice. This indicates that, in equilibrium situations, little overhang is found to be present.
} 
(a)

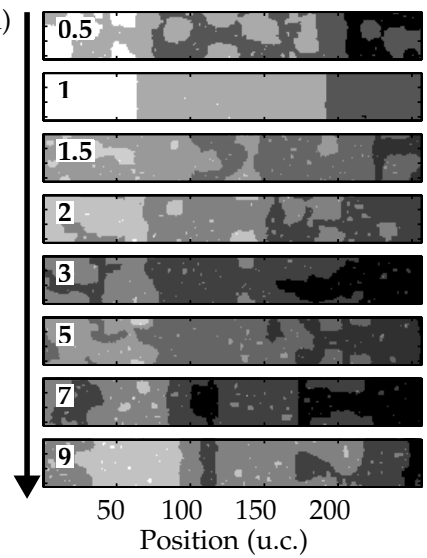

(b)

(c)

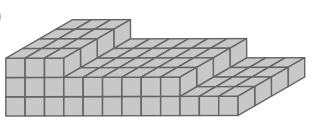

(d)

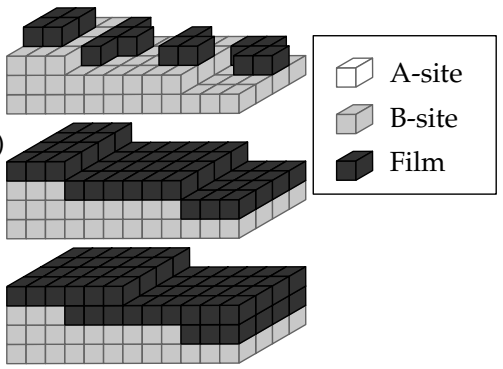

Figure 5.2: kMC simulation surface morphologies on a singly B-site terminated template, shown in (a). The time evolution of the morphology is depicted after deposition of the volume equivalent of $0.5,1,1.5,2,3,5,7$ and 9 monolayers. The stepped terrace structures and morphologies are indicated by the schematics depicted next to the results, (b) the template, (c) after $\sim 0.5$ monolayer of deposition islands form, (d) after $\sim 1$ monolayer a complete layer forms and (e) subsequent growth occurs in a step-flow like growth mode.

\subsubsection{Simulations on a simple termination template}

Results of kMC simulations on a singly B-site terminated template are depicted in Fig. 5.2(a). A schematic of the singly terminated template with two terraces steps is shown in Fig. 5.2(b). The growth on simulated B-site terminated areas $\left(E_{S}^{B}=\right.$ $1.3 \mathrm{eV})$, occurred in a layer-by-layer growth mode. The initial monolayer island formation, which occurred after depositing 0.5 monolayers, is shown schematically in Fig. 5.2(c). The islands coalesced and formed a closed layer after deposition one monolayer of material. The surface was now terminated by a layer of film material and $E_{S}$ equals $E_{S}^{\text {film }}$ on the entire surface $(1.1 \mathrm{eV})$, as depicted in Fig. 5.2(d).

Subsequent growth of additional film material on a film covered surface is identical to homoepitaxial growth; the energy barriers $E_{S}$ and $E_{N}$ remained fixed. Growth occurred in a step-flow-like growth mode, as depicted in Fig. 5.2(e). In the simulations, some island formation occurred. The islands were relatively large, but no second layer nucleation was observed. These large islands coalesced with terrace step edges. This resulted in meandering step edges.

The simulated growth behavior agreed with the experimentally observed initial growth of $\mathrm{SrRuO}_{3}$ on B-site terminated substrates, like $\mathrm{TiO}_{2}$ terminated $\mathrm{SrTiO}_{3}$. After completion of the initial layer, the diffusivity was found to be increased, resulting in step-flow like growth. Some island formation still occurred, resulting in meandering step edges. This step meandering was also observed by Rijnders [99] for simulated growth of $\mathrm{SrRuO}_{3}$. The meandering is more pronounced in this work, possibly due to the use of slightly different simulation parameters. 


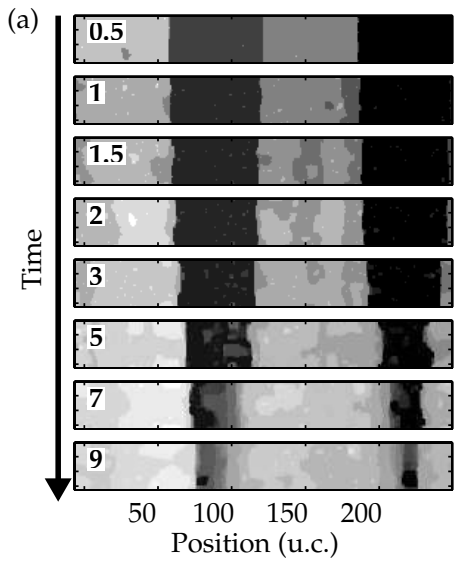

(b)

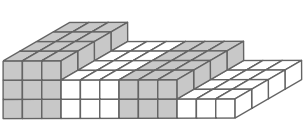

(c)

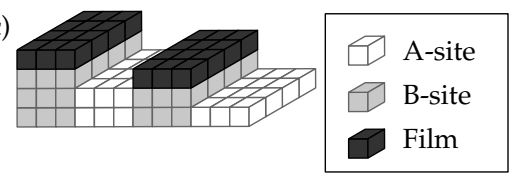

(d)

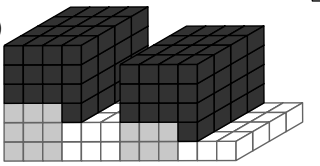

Figure 5.3: kMC simulation surface morphologies on an ordered mixed terminated surface, in (a). The time evolution of the morphology is depicted after deposition of the volume equivalent of $0.5,1,1.5,2,3,5,7$ and 9 monolayers. A schematic (b) of the ordered $50 \% \mathrm{~A}$ and $50 \% \mathrm{~B}$-site surface termination. Initial nucleation and growth (c) occurred preferentially on the B-site areas with $\mathrm{E}_{\mathrm{S}}^{\mathrm{A}}<<\mathrm{E}_{\mathrm{S}}^{\mathrm{B}}$. After completion of a closed film layer on the B-site area (d), nucleation and growth occurred preferentially on the film covered area, $\mathrm{E}_{\mathrm{S}}^{\mathrm{A}}<\mathrm{E}_{\mathrm{S}}^{\text {film }}$

\subsubsection{Simulations on ordered termination templates}

In Fig. 5.3(a), simulation results on an ordered mixed terminated template are shown. A schematic of the mixed template is shown in Fig. 5.3(b). Two terrace steps were simulate and the ratio of A/B-site termination was set to $50 \%$.

The energy barrier were set, as given in Table $5.1\left(\mathrm{E}_{\mathrm{S}}^{\mathrm{A}}=1.0 \mathrm{eV}\right)$, where $\mathrm{E}_{\mathrm{S}}^{\mathrm{A}}<<$ $E_{S}^{B}$. These settings resulted in a higher surface diffusivity on A-site compared to B-site. Therefore, the nucleation probability was higher on B-site compared to Asite. This resulted in all of the deposited material to initially nucleate and grow on the B-site areas. After the deposition of the volume equivalent of 0.5 monolayers the B-site terminated areas were completely covered with film material, as schematically indicated in Fig. 5.3(c). The initial growth on an ordered mixed terminated template showed that the film material preferentially nucleated on the B-site surfaces areas.

The growth observed after completely covering the B-site areas with film material was determined by the difference between $E_{S}^{A}$ and $E_{S}^{\text {film }}$. This difference was smaller compared to the difference between $E_{S}^{A}$ and $E_{S}^{B}$. The wires grew both in width and in height during subsequent deposition. Material was deposited both on the nanowires as on the A-site terminated substrate areas. The nucleation probability was the highest on the film covered areas. That includes the vertical edges of the nanowires which are formed. Therefore, nanowires also grew in width, as schematically indicated in Fig. 5.3(d)

Upon depositing several monolayer of film material, the nanowires grew closer 
together and islands started to form on A-site terminated areas. Initially, after deposition of 1 to 3 monolayers these islands were small. However, larger islands appeared over time. The islands showed a form of Oswald ripening with the large wire features. After simulating the deposition of roughly 10 monolayer, the nanowires coalesced and a continuous film was formed.

\subsubsection{Tuning simulated self-organization}

In literature experimental values of $\mathrm{E}_{\mathrm{A}}$ are obtained between 0.5 and $2 \mathrm{eV}$. ${ }^{[99,108]}$ However, this activation barrier does not give direct information on for example $\mathrm{E}_{\mathrm{N}}$ and $\mathrm{E}_{\mathrm{es}}{ }^{3}$. Moreover, the $\mathrm{E}_{\mathrm{S}}$ difference between A-site and B-site termination is unknown.

In order to study the effect of $E_{S}^{A}$ on the simulated growth of nanowires, the $E_{S}^{A}$ parameter was varied between 0.9 and $1.0 \mathrm{eV}$. The results are shown in Fig. 5.4. At $E_{S}^{A}=1.0 \mathrm{~V}$ the wire evolution was similar to the results shown in Fig. 5.3(a) and a lateral growth speed of $\sim 0.35$ u.c./ second was found. ${ }^{4}$ When $\mathrm{E}_{\mathrm{S}}^{\mathrm{A}}$ was reduced, the lateral growth speed was decreased to 0.15 and 0.12 u.c. / second for $E_{S}^{A}=0.95$ $\mathrm{eV}$ and $\mathrm{E}_{\mathrm{S}}^{\mathrm{A}}=0.9 \mathrm{eV}$ respectively. This lateral growth speed is indicated in Fig. 5.4 by the dotted lines.

The evolution of the wire edge position appears to be asymmetric with respect to the initial step-down and step-up sides of the B-site terminated area. This was most clearly observed in the evolution of $\mathrm{E}_{\mathrm{S}}^{\mathrm{A}}=0.95 \mathrm{eV}$ in Fig. 5.4(b), where the right side of wire shape grows slower than the left side. However, in some simulation the opposite is found. These asymmetries were attributed to small island formation close to the nanowire. These islands coalesced with the wire features and resulted in a small variation in the lateral growth speed of the nanowires.

\subsubsection{Time evolution of self-organized nanowire growth}

The simulated growth of nanowires on ordered mixed terminated templates, as shown in Fig. 5.3(a), showed simultaneous growth of the features in height (vertical) and in width (lateral). The lateral growth eventually resulted in coalescence of the nanowires. Using similar simulation parameters as used for the simulations shown in Fig. 5.3, the time evolution of the wire-height and width ${ }^{5}$ are shown in Fig. 5.5. A grid of 512x128 u.c. ${ }^{2}$ was used and three B-site areas of $\sim$ 56 u.c. in width covering about a third of the sample surface were used as a starting template.

Initially, the B-site terminated areas were covered with film material, while the A-site termination remained uncovered. The first complete layer of film material on the B-site terminated area was formed after $\sim 10$ seconds of deposition $(0.1$

\footnotetext{
${ }^{3}$ No significant change in wire growth was observed for $\mathrm{E}_{\mathrm{es}}$ values in the range of -0.15 to $0.15 \mathrm{eV}$.

${ }^{4}$ The lateral growth speed was calculated from the moment the wires started to grow in width (60 seconds).

${ }^{5}$ The wire-width and height were obtained from height maps by localizing peaks after applying a Sobel type edge detection algorithm.
} 


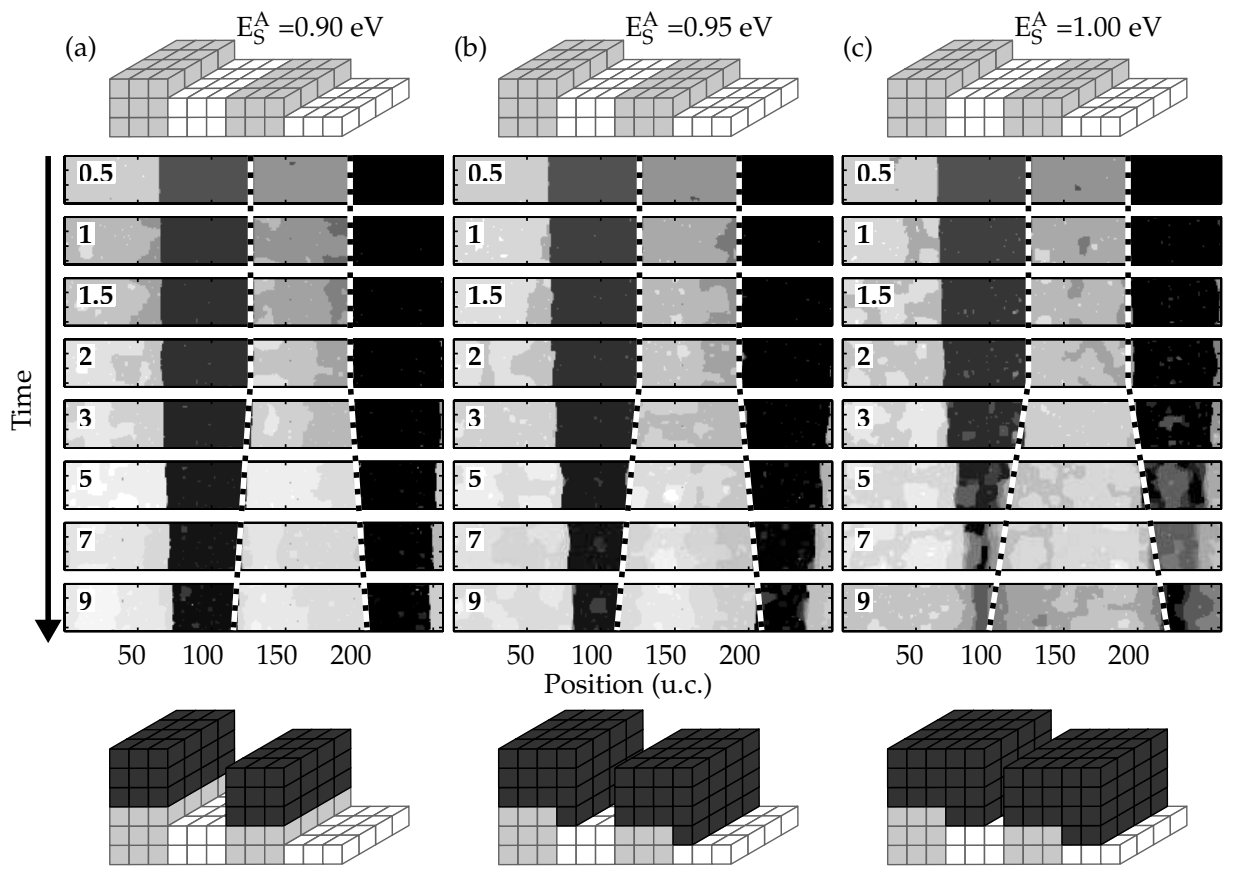

Figure 5.4: $\mathrm{kMC}$ simulation surface morphologies on ordered mixed terminated surfaces with equal coverage of $\mathrm{A}$ and B-site, similar to the simulation results shown in Fig. 5.3. Three values of $\mathrm{E}_{\mathrm{S}}^{\mathrm{A}}$ were simulated, $0.9 \mathrm{eV}(\mathrm{a}), 0.95 \mathrm{eV}(\mathrm{b})$ and $1.0 \mathrm{eV}$ (c). A larger $\mathrm{E}_{\mathrm{S}}^{\mathrm{A}}$ value reduced the diffusivity on A-site terminated surface areas, moreover the ratio between $\mathrm{E}_{\mathrm{S}}^{\mathrm{A}}$ $+E_{N}$ and $E_{S}^{\text {film }}$ was altered which changed the behavior near the edge of a nanowire. The stepped terrace structures and termination configurations are indicated by the schematics on the top of the figure and the resulting nanowires are schematically drawn at the bottom of the figure. The time evolution of the morphology is depicted after deposition of the volume equivalent of $0.5,1,1.5,2,3,5,7$ and 9 monolayers.

u.c./second). The growth speed remained constant up to 50 seconds of deposition time where 5 u.c. high wires were formed. After 50 seconds the wires started to grow in width. The vertical growth speed was reduced to $\sim 0.03$ u.c./second. The growth in width occurred at a rate of $\sim 0.3$ u.c./second, but was not linear in time. The volume of deposited material increased linearly, as no evaporation was taken into account (not shown).

This behavior near 50 seconds of deposition can be described by a simple one-dimensional model. A critical nanowire height is defined, similar in concept to the critical island radius for second layer nucleation ${ }^{[82]}$, discussed in section 5.1.1. The lateral growth of nanowires is considered as a form of second layer nucleation. In this model, in the first 50 seconds, there is no lateral growth; i.e. no nucleation on the nanowire sides. The vertical growth rate scales linearly with incident flux F: $h(t)=F t$. The wire width, $w(t)$ and spacing, $s(t)$, remains constant 
(a)

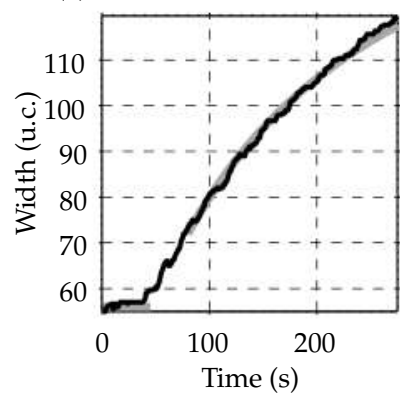

(b)

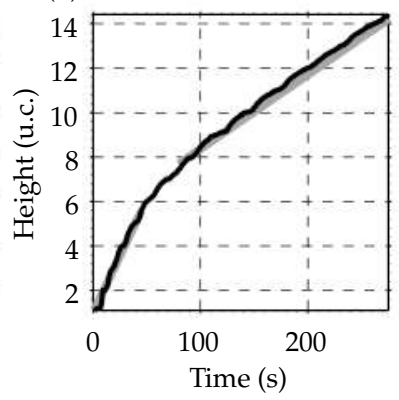

(c)

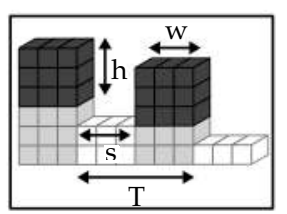

Figure 5.5: Nanowire growth evolution of width (a) and height (b). A lattice grid of $512 \times 128$ u.c. $^{2}$ was used with $1 / 3$ covered by B-site surface termination. Three B-site areas of $\sim 56$ u.c. in width and three A-site areas of $\sim 114$ u.c. in width were set as template. During growth, the width remained constant until roughly 50 seconds. The height initially increased quickly, but as the wires started to grow in width, the vertical growth speed decreased. The five-point running average was applied to the data, in order to smoothen outliers caused by the edge finding algorithm. The results of a simple 1-dimensional model are shown in light gray (see text). The main parameters of this model are indicated in (c): wire width, w, height, $h$, spacing, s and the terrace width, $T$.

up to 50 seconds. By assuming that after this point nucleation on the nanowire sides occurs, the interlayer mass transport is limited and Eq. (5.4) can be formulated. The growth rate of the wire spacing, $d s / d t$ is determined by the ratio of the amount of material deposited in between the wires, $F * s(t)$ and height of the wire. The wire width equals $T-s(t)$, where $\mathrm{T}$ is equal the total width of the $\mathrm{A}$ and B-site areas, typically the terrace width. These parameters are drawn in the schematic in Fig. 5.5(c).

$$
\frac{d h(t)}{d t}=F \quad \text { and } \quad \frac{d s}{d t}=-F \frac{s(t)}{h(t)} \quad \text { and } \quad \frac{d w}{d t}=-\frac{d s}{d t}
$$

By defining $h(0)=h_{\text {crit }}$ and $s(0)=s_{\text {crit }}$, Eq. (5.4) can be solved, giving Eq. (5.5).

$$
h(t)=F t+h_{\text {crit }} \quad \text { and } \quad s(t)=\frac{s_{\text {crit }} h_{\text {crit }}}{F t+h_{\text {crit }}} \quad \text { and } \quad w(t)=T-s(t)
$$

Results of this model are plotted in light gray in Fig. 5.5. This simple model shows good agreement with the simulation results, before and after the growth transition occurs.

\subsubsection{Simulated growth on other templates}

With the current parameter set, growth on completely B-site terminated templates and 50/50 mixed templates was successfully simulated. Simulated growth 

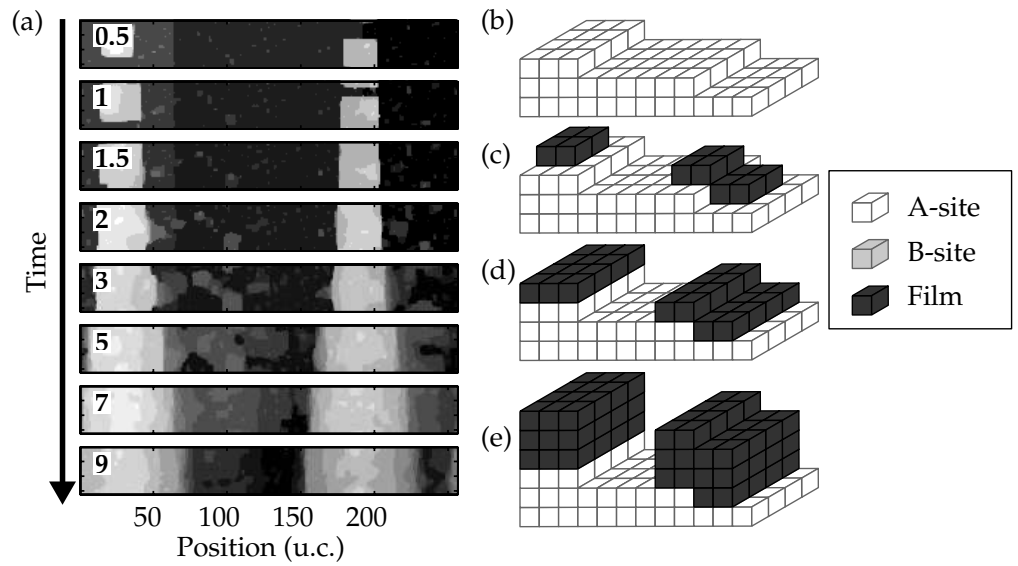

Figure 5.6: kMC simulation surface morphologies on a singly A-site terminated template, shown in (a). The time evolution of the morphology is depicted after deposition of the volume equivalent of $0.5,1,1.5,2,3,5,7$ and 9 monolayers. The stepped terrace structures and morphologies are indicated by the schematics depicted next to the results, (b) the template, (c) after $\sim 0.25$ monolayer of deposition islands form, (d) after $\sim 0.5$ monolayer (e) subsequent growth occurred similar to nanowire growth.

results agreed with experimentally observed growth. In chapter 3, various surface termination templates are proposed and measured for $\mathrm{DyScO}_{3}$ (110) substrates. Special focus is aimed at creating a perfectly A-site singly terminated template in section 3.4. $\mathrm{SrRuO}_{3}$ growth on artificially created DyO terminated surfaces resulted in island formation, which was attributed to non-perfect DyO termination, as observed by surface $\mathrm{X}$-ray diffraction.

Using kMC simulation, growth on perfect singly terminated A-site templates can be simulated and the simulations can be used to predict the growth of $\mathrm{SrRuO}_{3}$ on such A-site surfaces. The simulation results of growth on A-site terminated templates are shown in Fig. 5.6(a). The starting template morphology was set similar to the perfect B-site template, Fig. 5.2(b) and is depicted in Fig. 5.6(b). Here $E_{S}$ was set to $E_{S}^{A}$ on the entire surface. The diffusivity was high; only two islands formed after the deposition of the volume equivalent of 0.5 monolayers, as also indicated in the schematic in Fig. 5.6(c). One island started to nucleate at a step edge (right), whereas a second island nucleated on a terrace (left). Adatoms attached to these islands had an $E_{S}$ value equal to $E_{S}^{\text {film }}$. The islands formed a termination template where areas of $E_{S}^{A}$ and $E_{S}^{\text {film }}$ were obtained, as indicated in Fig. 5.6(d). This template was nearly identical to the template observed in Fig. 5.3(c)

Subsequent growth occurred in a way very similar to the growth on mixed terminated templates. The initial islands grew in both $\mathrm{x}$ and $\mathrm{y}$ directions, but due to the limited height of the simulation grid, the top and bottom of the island coalesced and appeared like a wire. The resulting patterns after the simulated deposition of 9 monolayer showed nanowire formation, as drawn in the schematic 
in Fig. 5.6(e). This indicates that with the current simulation parameters, a form of nanowire growth is expected to occur during $\mathrm{SrRuO}_{3}$ growth on perfectly Asite singly terminated substrates.

\subsection{Discussion}

Using an adapted SOS model, self-organization during growth on mixed terminated surfaces can be simulated, by introducing local differences in the diffusivity $\left(E_{S}\right)$. At sufficiently high temperatures, a small $E_{S}$ difference $(\sim 0.3 \mathrm{eV})$ will result in self-organized growth. Simulation results agreed qualitatively with experimental data, by assuming a low value of $E_{S}$ on simulated A-site surface termination compared to $E_{S}$ values on B-site or film covered areas: $E_{S}^{A}<E_{S}^{\text {film }} \leq E_{S}^{B}$. Wang et al. ${ }^{[110]}$ report adsorption of $\mathrm{Sr}$ and $\mathrm{Ti}$ favors $\mathrm{TiO}_{2}$ surface termination over SrO. Moreover, Eglitis and Vanderbilt ${ }^{[111]}$ report a higher surface energy for $\mathrm{TiO}_{2}$ termination compared to $\mathrm{SrO}$, indicating wetting of $\mathrm{TiO}_{2}$ is preferred. Therefore, the assumption $\mathrm{E}_{\mathrm{S}}^{\mathrm{B}}>\mathrm{E}_{\mathrm{S}}^{\mathrm{A}}$, is validated, for the $\mathrm{SrTiO}_{3}$ case. ${ }^{6}$

The selected parameters used for the $\mathrm{kMC}$ simulations, provide the expected growth modes on singly terminated B-site templates; the initial growth occurred in a layer-by-layer growth mode. Growth of the film material in the simulation occurred in a nearly step-flow-like growth mode, where the diffusion length was of the order of the size of the original B-site terminated areas. This agrees well with experimental growth results. Based on these results $E_{S}^{B}, E_{S}^{\text {film }}$ and $E_{N}$ were kept constant and diffusivity on A-site termination was initially assumed to be the main factor in determining the time evolution of the nanowire height and width.

Experimental data show that the $\mathrm{SrRuO}_{3}$ nanowires on $\mathrm{DyScO}_{3}$ coalesce after roughly $20 \mathrm{~nm}$ of $\mathrm{SrRuO}_{3}$ was deposited in 25 minutes (13 pm/second), see section 4.2.3. In the case of $100 \mathrm{~nm}$ wide wires, separated by $100 \mathrm{~nm}$, this corresponds to a vertical growth rate of $67 \mathrm{pm} / \mathrm{second}$ or 0.16 u.c. / second. This agrees with the growth speeds obtained using the simulations. ${ }^{7}$ However, experimental data also show that nanowires mimic the exact termination template up to 600 seconds of deposition, while the simulations show that already after 50 seconds of deposition, the wires start to grow laterally. ${ }^{8}$

This perfect vertical growth is modeled by defining a critical nanowire height. This height is similar in concept to the critical island size for second-layer nucleation in normal layer-by-layer growth. ${ }^{[82]}$ When nucleation results in an islands of at least the critical size, second layer nucleation starts to occur. The onset of second layer nucleation is calculated to be very sharp. ${ }^{[82]}$ If one assumes that the edge of the wire is similar to a vertical terrace and the height of the edge becomes of the order of the critical nanowire height: interlayer mass transport between

\footnotetext{
${ }^{6}$ No such studies are found for $\mathrm{DyScO}_{3}(110)$ surfaces.

${ }^{7}$ The possible initial growth of $\sim 5$ u.c. of nanowire as observed in the simulations does not significantly effect this estimation ( 0.18 u.c. / second).

${ }^{8} \mathrm{~A}$ similar growth speed was used in both experiments and simulations.
} 
the nanowire and the lower lying substrate is limited. This would reduce the vertical growth of the nanowires to material deposited on the wire. At this critical thickness, the lateral growth speed would increase, as is also observed in the simulations. Using this model, the observed simulated evolution of the wire height and width can be well-described, as shown in Fig. 5.5. The point or film thickness at which the wires started to grow laterally did not seem to be influenced by $E_{S}^{A}$, as shown in Fig. 5.4. Indicating the critical thickness behavior was most likely re-

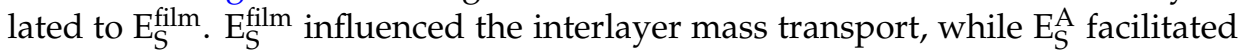
the inter-nanowire mass transport.

This critical thickness model could explain that small trenches observed in $\mathrm{SrRuO}_{3}$ growth on $\mathrm{SrTiO}_{3}$, for films even thicker than $20 \mathrm{~nm} .{ }^{[100]}$ As the amount of material in the trenches gets smaller with respect to the height of the nanowires, the material contributing to the lateral wire growth is reduced and the trenches might be stabilized in films above $20 \mathrm{~nm}$.

This initial growth behavior can be altered in the simulations to better reflect the experimental data, by changing the diffusion length on the film, i.e., $E_{S}^{\text {film }}$. This parameter is not studied in detail, as it was initially considered to be fixed. The simulations show formation of large islands and step-flow growth. Experiments indicate almost perfect step-flow growth mode and no island formation, indicating that the diffusivity in the simulations was set too low. ${ }^{9}$ A higher diffusivity also increases the critical island size, increasing the time perfect vertical growth occurs.

The simulation results obtained for nanowire type growth are used to study other types of surface termination templates, which are not readily obtained or studied experimentally. For example, the growth on singly terminated A-site templates shows interesting behavior. On single A-site termination, islands or nanowires of $\mathrm{SrRuO}_{3}$ are formed, very similar to growth on ordered templates of $\mathrm{A}$ and B-site surface termination. As $\mathrm{SrRuO}_{3}$ nucleates at the step edge, a termination pattern, similar to that of an ordered A/B situation, is formed. Effectively an ordered A/film surface termination pattern is obtained after growth of half a monolayer. Subsequent growth occurs on top of this initial layer, comparable to growth on ordered A/B templates.

\subsection{Conclusions}

Self-organized growth on ordered termination templates of perovskite-type oxides is qualitatively described using a three-dimensional kinetic Monte Carlo model. The model relies on a small difference in activation barrier for diffusion between $\mathrm{A}$ and $\mathrm{B}$-site terminated surface areas, $\mathrm{E}_{\mathrm{S}}^{\mathrm{B}}-\mathrm{E}_{\mathrm{S}}^{\mathrm{A}} \leq 0.3 \mathrm{eV}$ and requires a high surface diffusivity. The diffusion length on A-site termination should be larger than the spacing of B-site terminated areas. $\mathrm{E}_{\mathrm{S}}^{\mathrm{A}} \ll \mathrm{E}_{\mathrm{S}}^{\mathrm{film}}<\mathrm{E}_{\mathrm{S}}^{\mathrm{B}}$. This difference

\footnotetext{
${ }^{9}$ Increasing the diffusivity is computational intensive, due to the decrease in the time increment each simulation step represents.
} 
in diffusion lengths yields a higher nucleation probability on B-site compared to A-site terminated surfaces.

The simulations show a critical feature height, above which the vertical growth speed is reduced and the lateral growth speed is increased. The initial perfect vertical growth, but eventual coalescence of the nanowires is also observed experimentally. Experimental results indicate a higher critical thickness compared to the simulations. By increasing the diffusivity in the $\mathrm{kMC}$ simulations, a higher critical thickness might be obtained, for example by lowering $\mathrm{E}_{\mathrm{S}}^{\text {film }}$.

The observed sensitivity of $\mathrm{SrRuO}_{3}$ the local surface termination is not present in all material. The apparent sensitivity of $\mathrm{SrRuO}_{3}$ is expected to be related to the volatility of $\mathrm{RuO}_{x}$ and the termination conversion during the initial stages of growth. Similar behavior might be found in systems with other volatile species, like $\mathrm{CaRuO}_{3}, \mathrm{BiFeO}_{3}$ and $\mathrm{PbTiO}_{3}$.

\subsubsection{Outlook}

The type of SOS or kMC simulations performed in this work only take into account averaged interaction instead of the actual crystal growth. In literature reports, the number of interactions is increased, by simulating diffusion and nucleation of individual cations. For example by allowing only certain types of atoms to only move onto specific position of a predefined grid. This grid resembles, for example, a perovskite structure ${ }^{[95]}$. However, such models require defining many interaction energies, which are not well-known. By using molecular dynamics calculations, these interaction can be calculated and used as input for Monte Carlo simulations, resulting in hybrid models. ${ }^{[112,113]}$ Such hybrid models could provide a more detailed description the energy barriers for diffusion in complex oxide heteroepitaxy. 


\title{
Chapter 6
}

\section{Structural phase transition in ultra-thin $\mathrm{SrCuO}_{2}$ films}

\begin{abstract}
Epitaxial and atomically smooth ultra-thin $\mathrm{SrCuO}_{2}$ films are grown on $\mathrm{SrTiO}_{3}$ substrates using pulsed laser deposition. The structural and chemical aspects of these single-layer films of various thickness are characterized using in situ X-ray photoelectron diffraction (XPD) and photoelectron spectroscopy. A structural transformation is demonstrated from bulk-planar to chain-type $\mathrm{SrCuO}_{2}$, as the film thickness is reduced from 9 to 3 unit-cells, by comparing XPD scans to multiple-scattering electron diffraction simulations. This observation is in agreement with the recent theoretical prediction by Zhong et al. ${ }^{[114]}$ and opens new pathways for tuning the crystal structure in ultra-thin films of polar cuprates.
\end{abstract}

Published as journal article: B. Kuiper, D. Samal, D.H.A. Blank, J.E. ten Elshof, G. Rijnders and G. Koster. APL Materials 1, 042113 (2013). ${ }^{[115]}$ 


\subsection{Introduction}

By reducing the film thickness, creating nanopatterns and thereby the dimension, one can tune the properties of various material systems. In case of oxides, a change in properties typically coincides with an alternation of the crystal structure or geometrical shape. The structural analysis and quantification of ultra-thin films and nanostructures is of high interest in this regard. In this chapter, the use of X-ray photoelectron diffraction (XPD) for the analysis of oxygen sublattice structures in ultra-thin films is discussed on a model system: $\mathrm{SrCuO}_{2}$. In the $\mathrm{SrCuO}_{2}$ case, a clear shift is expected to be observed using XPD. Knowledge gained by analyzing this model system is paramount for the analysis and understanding of more subtle changes typically expected in ultra-thin oxide films, like $\mathrm{SrRuO}_{3}$, discussed in chapter 7 .

In recent years there has been a dramatic increase in designing, growing and characterizing novel complex oxide materials with atomic precision that give rise to enhanced or new functionalities. The study of the resulting atomically engineered layers and interfaces has proven to be a promising field of research, with the opportunity to manipulate and control various degrees of freedom, e.g., electronic, lattice, spin and orbital. Specifically, for the case of $\mathrm{ABO}_{3}$ complex oxide thin films, the shape, rotations and distortions of the $\mathrm{BO}_{6}$ oxygen octahedral due to epitaxial strain and/or lattice defects play a crucial role in the resulting physical properties. ${ }^{[116,117]}$ Moreover, the structure of the oxygen sublattice at the interface between polar and non-polar materials, e.g., $\mathrm{LaAlO}_{3}-\mathrm{SrTiO}_{3}{ }^{[10]}$ plays a vital role in many of the exotic interfacial phenomena. Therefore, the local structure at interfaces and in ultra-thin layers is of great importance in such systems and needs to be explored precisely.

Oxygen atomic positions in such materials can be studied using various techniques, e.g., by measuring the oxygen positions and tilt patterns using $\mathrm{X}$-ray diffraction ${ }^{[116,117]}$ or by using transmission electron microscopy ${ }^{[118]}$. Alternatively it is possible to probe the local structure using electron diffraction ${ }^{[119]}$ or X-ray photoelectron diffraction (XPD). ${ }^{[120-123]}$ In particular, the latter technique, $\mathrm{XPD}$, is highly suitable to study ultra-thin films, due to its high surface sensitivity and element specific structural information. Moreover, it does not require any sample preparation, it can be applied to films with less than one full layer of coverage and it is a non-destructive method. XPD has been successfully applied to various oxide thin film systems, which provides unique information about the oxygen displacement, even with displacements of a fraction of an angstrom. [121] For example XPD has been used to analyze the surface structure of $\mathrm{SrTiO}_{3}{ }^{[120,124]}$ by comparison of experimental data with multiple-scattering (MS) simulations. Here this technique is used to study this structural transformation in ultra-thin polar infinite-layer $\mathrm{SrCuO}_{2}$.

The infinite layer tetragonal $\mathrm{SrCuO}_{2}(\mathrm{a}=\mathrm{b}=3.926 \AA, \mathrm{c}=3.432 \AA)^{[8]}$ is one of the parent oxide structures in the cuprate family that hosts high $\mathrm{T}_{C}$ superconductivity. ${ }^{[7,125,126]}$ Its structure can be considered as an oxygen deficient perovskite, 

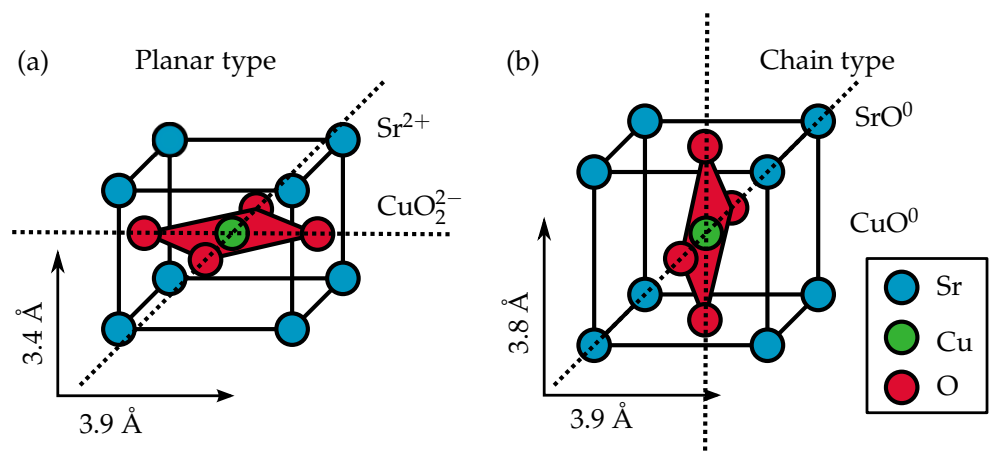

Figure 6.1: Schematic drawing of the crystal structure of (a) planar or infinite layer $\mathrm{SrCuO}_{2}$ and (b) chain-type $\mathrm{SrCuO}_{2}$. Dotted lines indicate the both directions of the $\mathrm{CuO}_{4}$ plaquettes.

where one oxygen atom is missing in the $\mathrm{Sr}^{2+}$ plane. Essentially, its structure is planar and can be viewed as an alternative stacking of $\mathrm{Sr}^{2+}$ and $\mathrm{CuO}_{2}^{2-}$ planes ${ }^{\text {[127]; }}$ where each $\mathrm{Cu}^{2+}$ ion is four-fold coordinated to $\mathrm{O}^{2-}$ ions forming $\mathrm{CuO}_{4}$ plaquettes. Since each alternative constituent atomic plane has a formal charge of $+/-2 \mathrm{e}$; it leads to a built-in electrostatic potential, which increases with the $\mathrm{SrCuO}_{2}$ thickness. ${ }^{[127]}$

The recent study by Zhong et al. ${ }^{[114]}$ on ultra-thin films of $\mathrm{SrCuO}_{2}$ predicts a structural transformation from bulk planar to a chain-type structure upon reducing the thickness below $\sim 5$ unit-cells, that relieves the built-in internal electrostatic potential in $\mathrm{SrCuO}_{2}$. Effectively this phase transformation is caused by an atomic reconstruction, where one oxygen atom is moved from the $\mathrm{CuO}_{2}^{2-}$ plane to the $\mathrm{Sr}^{2+}$ plane; thereby making the charge neutral $\mathrm{SrO}$ and $\mathrm{CuO}$ atomic planes. The modified chain-type structure can be viewed as if the $\mathrm{CuO}_{4}$ plaquettes are stacked along a perpendicular direction as compared to that in the case of planar one. A schematic of chain vs plane-type $\mathrm{SrCuO}_{2}$ layering and the local charge at the planes is shown in Fig. 6.1. In the process of rearrangement of oxygen ions, the c-axis lattice parameter of the chain-type $\mathrm{SrCuO}_{2}$ on $\mathrm{SrTiO}_{3}$ is predicted to be increased by $0.5 \AA$ as compared to the bulk-planar counterpart. In fact the recent study by D. Samal et al. ${ }^{[128]}$ demonstrated this effect, which clearly shows a change in the oxygen sublattice as a function of $\mathrm{SrCuO}_{2}$ thickness in $\mathrm{SrCuO}_{2}-$ $\mathrm{SrTiO}_{3}$ superlattice heterostructures. In addition, the work by Aruta et al. ${ }^{[129]}$ on $\mathrm{CaCuO}_{2}-\mathrm{SrTiO}_{3}$ superlattice heterostructures also hypothesized the possible formation of $\mathrm{CuO}$ chain-type layering at the interface, that in a way relieves the built-in electrostatic potential in $\mathrm{CaCuO}_{2}$.

Realization of artificially made chain-type structures in ultra-thin $\mathrm{SrCuO}_{2}$ layers will open new routes to design/engineer novel superconducting cupratehybrids, with alternation of chain and plane-type structures (the basic structural paradigm in cuprates) that can give more insight into the study of high Tc cuprates at a fundamental level. Moreover, a recent study suggests that the addition of a 
$\mathrm{SrCuO}_{2}$ epilayer strongly reduces the impurity scattering at the conducting interfaces in oxide $\mathrm{LaAlO}_{3}-\mathrm{SrTiO}_{3}$ heterostructures, opening the door to higher carrier mobility materials. ${ }^{[130]}$

Despite a great deal of interest in infinite layer ultra-thin polar cuprate films, no studies on the structure of bare/single layer ultra-thin films are found in the literature. Ultra-thin single-layer films should be much cleaner to study as compared to superlattice heterostructures made out of the same layers. In the case of superlattice heterostructures more complexity arises due to the addition of many interfaces that leads to chemical intermixing, interdiffusion, roughening etc. Here such bare thin films of $\mathrm{SrCuO}_{2}$ are studied.

In this chapter, the results of a detailed structural study on bare $\mathrm{SrCuO}_{2}$ layers in the ultra-thin limit, that essentially supports the prediction ${ }^{[114]}$ for a structural transformation from planar to chain-type with reducing $\mathrm{SrCuO}_{2}$ thickness. Thin films of 3 and 9 unit-cells in thickness are fabricated and studied, which are expected to respectively be below and above the critical thickness of 5 monolayers. By using XPD the precise change in the c-axis lattice parameter between the chain and the plane-type $\mathrm{SrCuO}_{2}$ layers is measured. MS electron diffraction calculations based on the program EDAC ${ }^{[36]}$ are used to simulate diffraction patterns that match the experimental data. A similar approach was used on a related cuprate, $\mathrm{CuO}$, in ultra-thin form, to successfully detect a tetragonal phase. ${ }^{\text {[131] }}$ Moreover, X-ray photoelectron spectroscopy (XPS) and (UPS) are used to quantitatively compare the film stoichiometry and account for the possible change in electronic structure between chain-type and planar $\mathrm{SrCuO}_{2}$.

\subsection{Experimental results and simulations}

Before investigating the predicted structural phase transition of $\mathrm{SrCuO}_{2}$ ultrathin films, first the growth of $\mathrm{SrCuO}_{2}$ on $\mathrm{SrTiO}_{3}$ substrates is studied using RHEED and AFM. Next XPD results of the bare $\mathrm{SrTiO}_{3}$ substrates are discussed and finally $\mathrm{SrCuO}_{2}$ films above and below the critical thickness are compared using XPD, XPS, UPS and MS simulations.

\subsubsection{Substrate preparation and $\mathrm{SrCuO}_{2}$ film growth}

TM-AFM micrographs of two substrates are shown in Fig. 6.2(a,b) indicating an atomically smooth surface. No signs of mixed termination were observed, indicating a single $\mathrm{TiO}_{2}$ surface termination. Both samples had a miscut of about $0.05^{\circ}$. Figure 6.2(c) shows the time evolution of the RHEED specular reflection for $\mathrm{SrCuO}_{2}$ growth on both substrates. One clear oscillation was observed, while the overall intensity decreased to a fixed value in about 40 seconds. For both samples the first maximum in the specular intensity coincided with the first minimum in the full-width-half-maximum, indicating completion of one layer of $\mathrm{SrCuO}_{2}$. The growth speed was estimated using the initial oscillation: 13 pulses per unit-cell layer for the 9 unit-cell sample and 14 pulses per unit-cell layer for the 3 unit-cell 

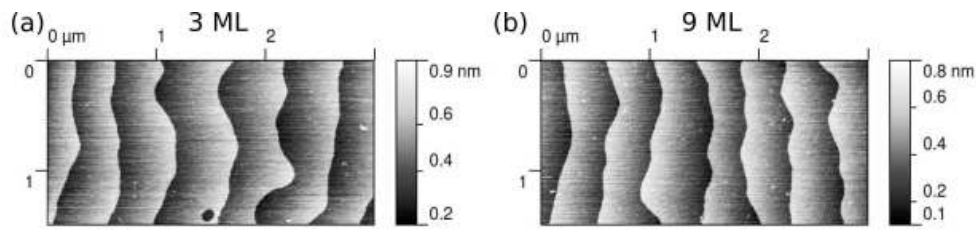

(c)
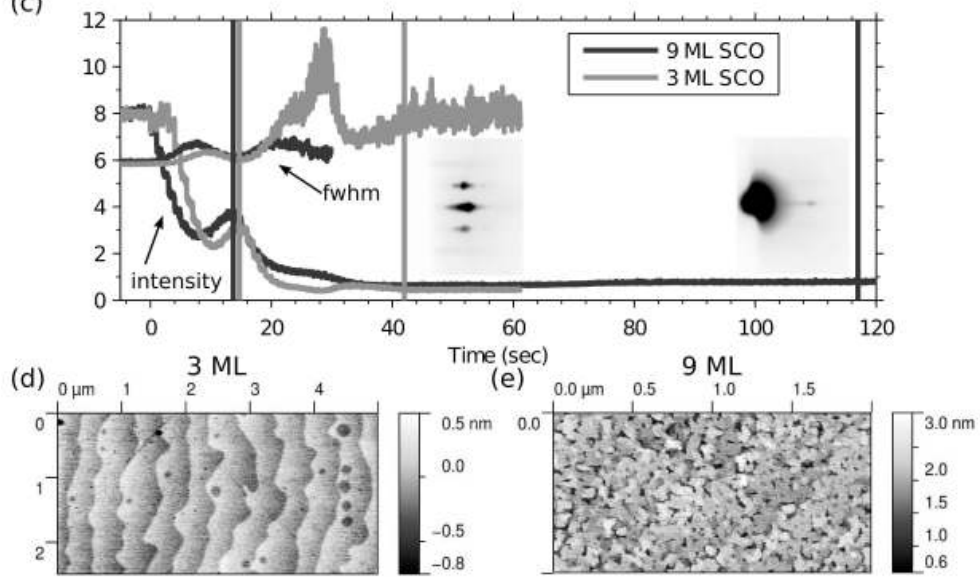

Figure 6.2: Growth and morphology of $\mathrm{SrCuO}_{2}$ films on $\mathrm{Nb}$ doped $\mathrm{SrTiO}_{3}$. Image (a) and (b) show TM-AFM data of substrates used for the deposition of 3 and 9 layers of $\mathrm{SrCuO}_{2}$. In (c) the RHEED specular intensity and full width half maximum evolution during growth is shown of both films is shown. (d) and (e) show the resulting film morphologies. Insets in (c) are RHEED images taken after growth of $\mathrm{SrCuO}_{2}$.

sample. Deposition was stopped at 117 and 42 seconds respectively. AFM images shown in Fig. 6.2(d,e) had root-mean-square (RMS) roughness values of 0.18 and $0.36 \mathrm{~nm}$ for 3 and 9 unit-cell films respectively, which was less than the c-axis parameter of $\mathrm{SrCuO}_{2}$, indicating atomically smooth surfaces. Both RHEED patterns shown in the insets of figure Fig. 6.2(c) are indicative of a two-dimensional/flat surface structure. However, a comparatively weaker intensity of the RHEED pattern corresponding to the 9 unit-cell sample was attributed to increased roughness at the atomic scale. ${ }^{[29]}$

\subsection{2 $\mathrm{SrTiO}_{3}$ substrate reference structure}

When measuring ultra-thin films of $\mathrm{SrCuO}_{2}$ using XPD, the top-most surface layers will contribute most to the observed signal. However, in the case of only 3 unit-cells of $\mathrm{SrCuO}_{2}$, contributions from the $\mathrm{SrTiO}_{3}$ substrate was visible in the observed photoemission spectra, see also section 6.2.4. To be able to separate possible substrate contributions to the photoelectron diffraction pattern, the structure of $\mathrm{SrTiO}_{3}$ was studied in exactly the same set-up as used for the $\mathrm{SrCuO}_{2}$ films. These $\mathrm{SrTiO}_{3}$ reference spectra were also used to confirm proper operation and analysis of the used XPD set-up, by comparison with results found in litera- 
(a)

Sr 3d

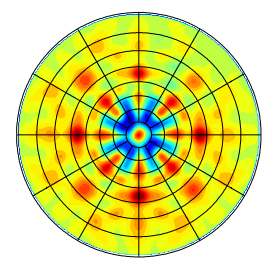

Ti $2 p$

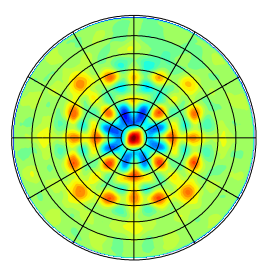

O 1s

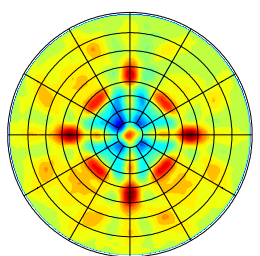

(b)
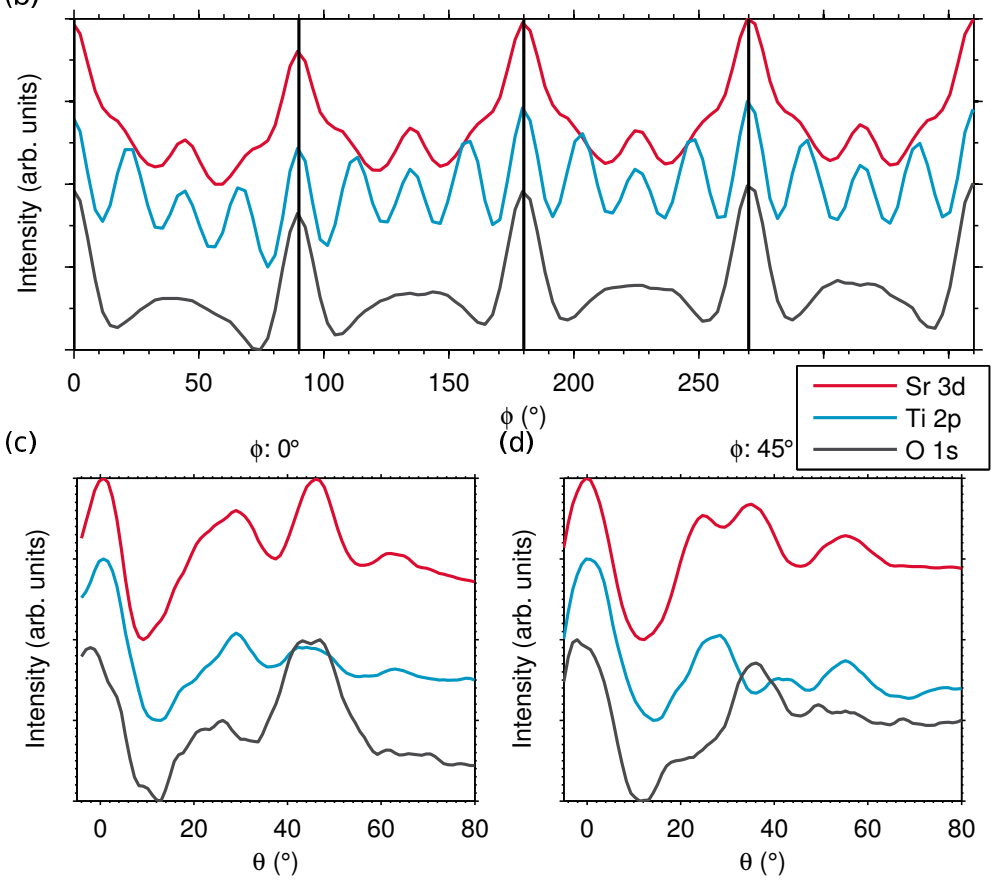

Figure 6.3: X-ray Photo-electron diffraction stereographic projections, (a) of a $\mathrm{TiO}_{2}$ terminated $\mathrm{Nb}$ doped $\mathrm{SrTiO}_{3}$ substrate for $\mathrm{Sr} 3 \mathrm{~d}$, Ti $2 \mathrm{p}$ and $\mathrm{O}$ 1s core level electrons using a $\theta$ stepsize of $1^{\circ}$ and a $\phi$ stepsize of $3^{\circ}$. Corresponding $\phi$-scans (b) and $\theta$-scans (c),(d) at $\phi$ equals $0^{\circ}$ or $45^{\circ}$ respectively. A three point moving average was applied in order to smoothen the data.

ture. ${ }^{[120,123,124]}$

Figure 6.3(a) contains full maps of Sr $3 \mathrm{~d}(133 \mathrm{eV})$, Ti $2 \mathrm{p}(486 \mathrm{eV})$ and $\mathrm{O} 1 \mathrm{~s}$ $(530 \mathrm{eV})$ core level binding energies recorded at $\theta$ step-sizes of $1^{\circ}$ and $\phi$ step-sizes of $3^{\circ}$. A linear spectral background was removed for every angle and the machine specific angular background was removed by subtracting a scaled dataset recorded at $1150 \mathrm{eV}$ (no peak present), see section 2.3.4. Clear four fold symmetry was observed as expected for cubic $\mathrm{SrTiO}_{3}$. Slight intensity deviations as a function of $\phi$ were caused by wobble of the sample manipulator. A correction for this wobble was applied to the $\theta$ axis. However, the intensity was also reduced, 

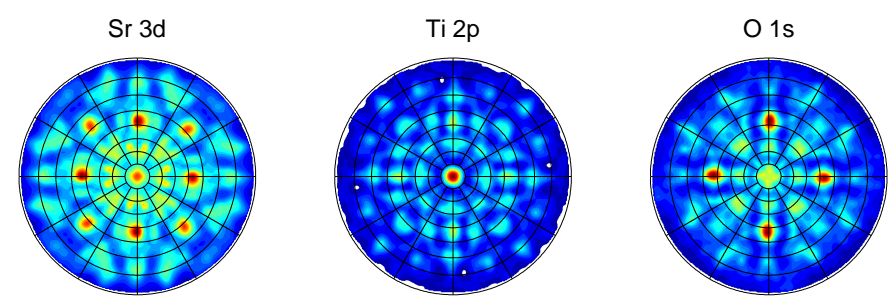

Figure 6.4: Simulated XPD patterns of $\mathrm{SrTiO}_{3}$ for $\mathrm{Sr} 3 \mathrm{~d}, \mathrm{~T} 2 \mathrm{p}$ and $\mathrm{O} 1 \mathrm{~s}$ emitter atoms. An EDAC parabolic cluster containing 236 atoms was used as input.

because the sample was not perfectly aligned with the focal point of the hemispherical analyzer and x-ray source for every angle. The highest signal to noise ratio was obtained from $\mathrm{O} 1 \mathrm{~s}$ photo-electrons. This was caused partially by the highest absolute count rate for $\mathrm{O}$ 1s in the spectrum and partially by diffraction effects. By applying a forward focusing ${ }^{[37,38]}$ analyses, which assumes enhanced scattering along atomic rows, the strongest peaks were observed for the lowest miller index direction: [001] $(\theta=0)$ and [101], [011], [101], [011] at $\theta=45^{\circ}$, see also section 2.3.4.

High resolution $\phi$-scans at $\theta=45^{\circ}$ shown in Fig. 6.3(b) revealed that in between the main peaks discussed before, several additional peaks were present. In a forward focusing approach these correspond to the [111] $\left(\phi=45^{\circ}\right),[121]\left(\phi=27^{\circ}\right)$ and [211] $\left(\phi=63^{\circ}\right)$ peaks. However, to predict the exact positions and peak shapes the forward focusing approach does not suffice. When for example the distance between atoms in an atomic row is reduced a hollow/volcano ${ }^{[132]}$ shape peak might appear instead of a Gaussian shaped peak, especially at high kinetic energies. Therefore, MS simulations were done using EDAC ${ }^{[36]}$ which use the entire structure to calculate the expected photoelectron diffraction pattern.

Such MS simulation results of a $\mathrm{TiO}_{2}$ terminated $\mathrm{SrTiO}_{3}$ substrate are depicted in Fig. 6.4. Simulated patterns ${ }^{1}$ matched the recorded data qualitatively. The peak positions were predicted and measured at similar values. However, relative intensity difference between peaks were different in measured data compared to simulations results. These difference can be partly attributed to the experimental $\theta$ background subtraction method, which was an approximation of the true background. Fine-tuning the simulation parameters might also improve the relative intensity differences. For the study of $\mathrm{SrCuO}_{2}$ in this chapter, focus is directed towards analyzing the peak positions, therefore this was not studied in more detail.

\footnotetext{
${ }^{1} \mathrm{~A}$ parabolic cluster of 236 atoms was used and a inner potential of $10.5 \mathrm{eV}$, an inelastic mean free path of $23 \AA$, maximum total angular momentum of 15 and a maximum inter-atom distance of $1.8 \mathrm{~nm}$ were set as input parameters. At absolute zero temperature the results converged after five iteration steps.
} 

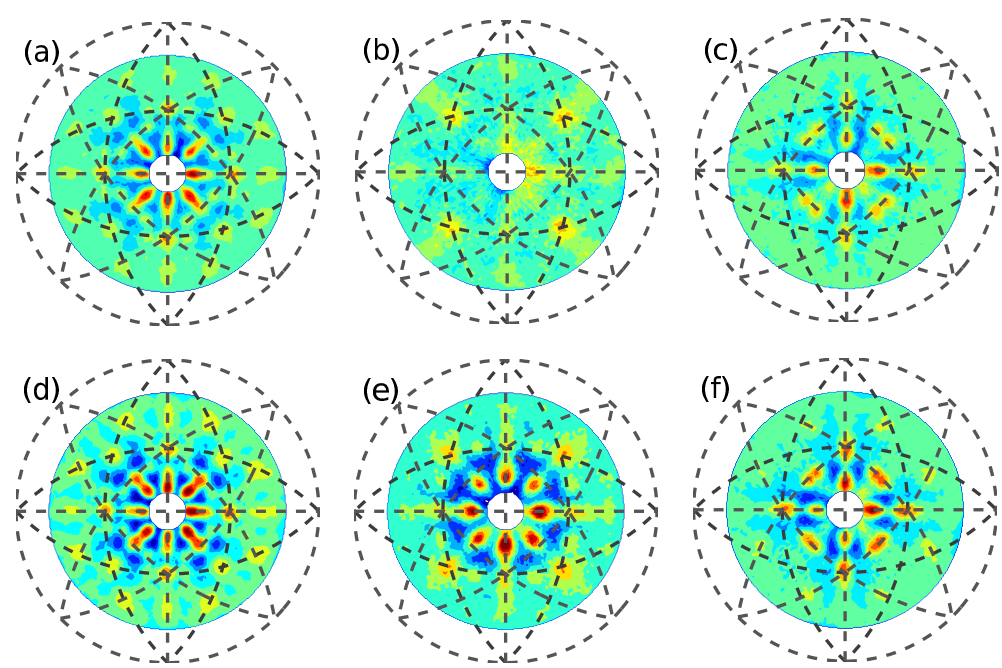

Figure 6.5: Stereographic projection of the intensity maps of (a-c) 3 layers of $\mathrm{SrCuO}_{2}$ on $\mathrm{SrTiO}_{3}$ and (d-f) 9 layers of $\mathrm{SrCuO}_{2}$. Sr 3d emission is show in (a,d), Cu $3 p$ in (b,e) and O 1s in $(c, f)$. The dotted lines are drawn as guides to the eye where the intersection correspond the zone axis direction for a simple cubic structure.

\subsection{3 $\mathrm{SrCuO}_{2}$ structural characterization by XPD}

Experimental intensity maps of a 3 mono-layer $\mathrm{SrCuO}_{2}$ film are shown in Fig. 6.5(ac) for $\mathrm{Sr} 3 \mathrm{~d}(134 \mathrm{eV}), \mathrm{Cu} 3 \mathrm{p}(77 \mathrm{eV})$ and $\mathrm{O} 1 \mathrm{~s}(531 \mathrm{eV})$. Similar stereographic projections for a 9 mono-layer $\mathrm{SrCuO}_{2}$ film are shown in Fig. 6.5(d-f). All patterns showed a clear four-fold symmetry. The $\mathrm{O} 1 \mathrm{~s}$ and $\mathrm{Sr} 3 \mathrm{~d}$ maps of the 3 and 9 layer film showed a similar general shape. For the 9 layer case the signal to noise ratio was higher than for the 3 layer $\mathrm{SrCuO}_{2}$ film, giving rise to a sharper diffraction pattern. This was observed most clearly in the $\mathrm{Cu} 3 p$ pattern. For $\mathrm{Cu} 3 p$ the peaks near $\theta=0^{\circ}$ were almost not observed. An outward shift $(\theta)$ was observed for all peaks, by analyzing the exact peak positions in the maps shown in Fig. 6.5.

Presented XPD results were recorded using X-ray generated photoelectrons at kinetic energies above $500 \mathrm{eV}$. As mentioned above, at such emission energies, forward scattering effects dominate. ${ }^{[37,38]}$ This allowed for direct analysis of the crystal structure. The main peak positions can be calculated based on these atomic rows. If for a simple cubic structure the forward scattering along the [001] direction is set at $\theta=0^{\circ}$ (out-of-plane): the [101] peak occurs at $45^{\circ}$ and the [111] at $54^{\circ}$. In the case of $\mathrm{SrCuO}_{2}$ the chain-type structure (c-axis $\sim 3.8 \AA$ ) has a main [101] peak at $\theta=46^{\circ}(\tan \theta=3.9 / 3.8)$ and a planar structure (c-axis $\sim 3.4 \AA$ ) at $\theta$ $=49^{\circ}(\tan \theta=3.9 / 3.4)$. A more detailed picture of these main peaks is given in Fig. 6.6, where the atomic rows of $\mathrm{Sr}$ atoms are highlighted with the matching $\theta$ angles for both planar and chain-type $\mathrm{SrCuO}_{2}$.

High resolution XPD $\theta$-scans of both $\mathrm{SrCuO}_{2}$ films are depicted in Fig. 6.7 for $\mathrm{Cu} 2 \mathrm{p}, \mathrm{Sr} 3 \mathrm{~d}$ and $\mathrm{O}$ 1s electrons. Clear shifts in $\theta$ peak positions in the $\mathrm{O} 1 \mathrm{~s}$ 


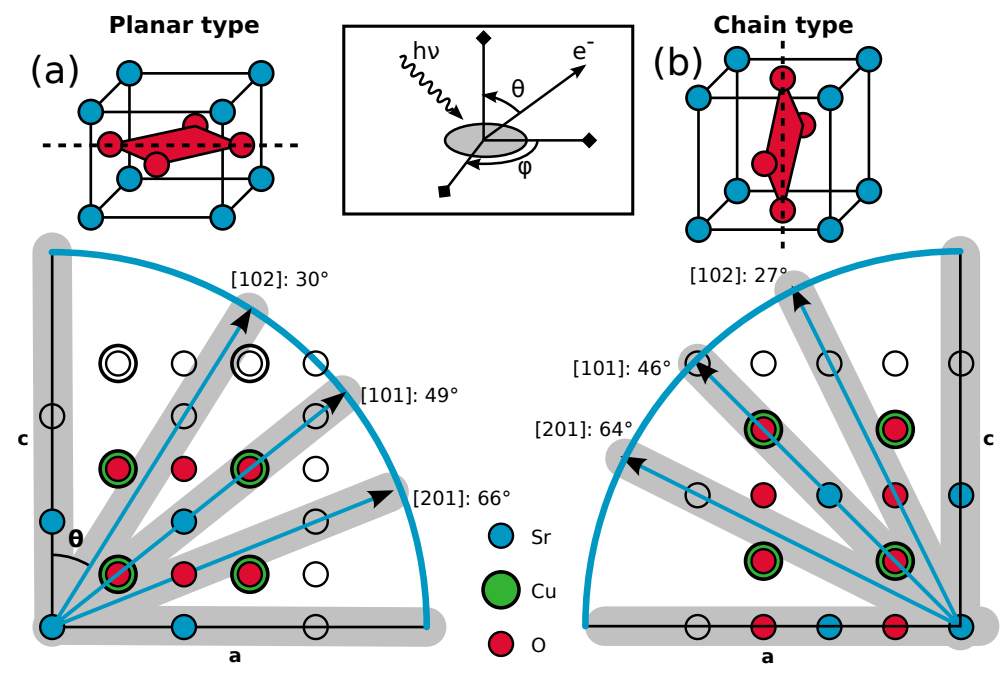

Figure 6.6: Schematic drawing of the crystal structure of (a) planar or infinite layer $\mathrm{SrCuO}_{2}$ and (b) chain-type $\mathrm{SrCuO}_{2}$. Strong forward scattering angles [101], [102] and [201] are shown for both structures and corresponding $\theta$ angles are calculated. The central inset schematically indicates the XPD measurement geometry, see also section 2.3.4.

and $\mathrm{Sr} 3 \mathrm{~d}$ scans were observed, when comparing the 3 unit-cells and 9 unit-cells $\mathrm{SrCuO}_{2}$ films. For clarity, the peak positions are indicated by vertical lines (blue for 9 unit-cell and red for 3 unit-cell). The main peak in the $\mathrm{O} 1 \mathrm{~s}$ spectrum for the 9 unit-cell film was found at $49.9 \pm 1^{\circ}$ and at $45.4 \pm 1^{\circ}$ for the 3 unit-cell $\mathrm{SrCuO}_{2}$ film. These angles corresponded to a c-axis of $\sim 3.8 \AA$ and $\sim 3.3 \AA$ respectively. Similar values were found for the $\mathrm{Cu} 3 \mathrm{p}$ scans. However for $\mathrm{Sr} 3 \mathrm{~d}$, the observed shift in $\theta$ angle was reduced. An overview of all measured peak position and calculated c-axis values is given in Table 6.1. An average $\theta$ shift of $\sim 3^{\circ}$ was observed when increasing the film thickness from 3 to 9 unit-cells. This is in accordance with the expected structural transformation.

Moreover, the experimental patterns were compared to MS simulations (green lines) based on the theoretical structures, as shown in Fig. 6.6. The Sr 3d scan showed a good agreement between experiment and simulation, both in the peak position and the peak shapes for planar and chain-type structures. The $\mathrm{O} 1 \mathrm{~s}$ simulation showed the main peak position at nearly the same value of $\theta$ as observed in the experimental data. However, at lower angles the simulations deviated from the experimental data for the chain-type structure. The $\mathrm{Cu} 3 \mathrm{p}$ simulations did not show good agreement with the experimental results, but the main experimental peak positions were still in good agreement with the forward scattering mechanism. Moreover, the rather flat and featureless experimental spectrum for the 3 unit-cell sample was at least qualitatively predicted by theory. 

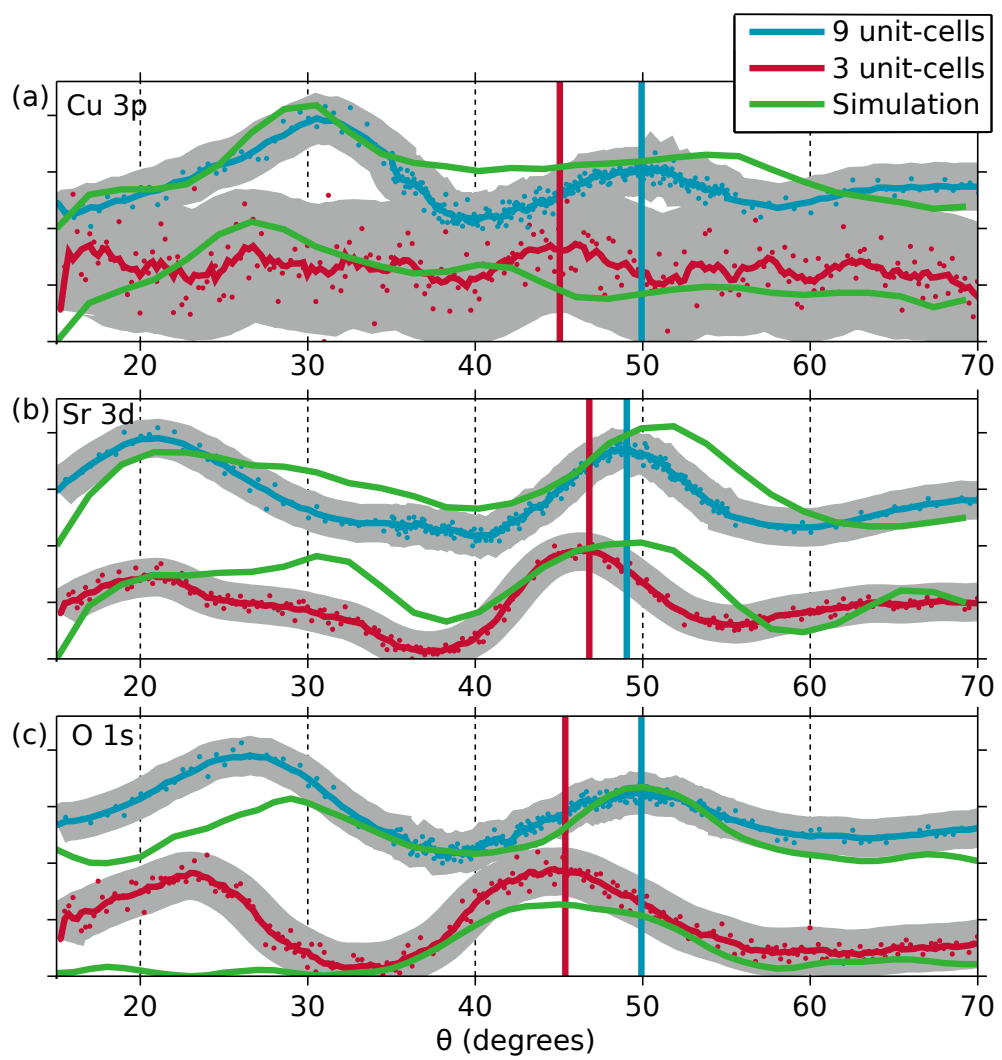

Figure 6.7: XPD measurements and simulations of $\mathrm{SrCuO}_{2}$ ultra-thin films of 3 and 9 unit-cells in thickness. $\theta$ scans are shown for (a) $\mathrm{Cu} \mathrm{3p}$, (b) Sr 3d and (c) O 1s. The dots are measured data points, similar colored solid lines are acquired by applying a 9 point moving average. EDAC simulation results are shown in green.

\subsubsection{Thin film photoemission}

Photoelectron spectra of both $\mathrm{SrCuO}_{2}$ films recorded at normal emission angle $\left(\theta=0^{\circ}\right)$ are shown in Fig. 6.8. Core level spectra of $\mathrm{Cu} 2 \mathrm{p}, \mathrm{O} 1 \mathrm{~s}, \mathrm{Sr} 3 \mathrm{~d}$ and Ti $2 \mathrm{p}$ of the substrate are plotted. The Ti $2 \mathrm{p}$ signal from the $\mathrm{SrTiO}_{3}$ substrate was used to correct the measured binding energies for sample charging effects (shifts in the observed energy value). For all individual scans a Shirley background was subtracted and the intensity normalized to the total area, in order to compare peak shapes. A Ti $2 p$ signal from the substrate was present in both films. Thus, the observed $\mathrm{Sr} 3 \mathrm{~d}$ and $\mathrm{O}$ 1s spectral lines contained electrons from both the $\mathrm{SrCuO}_{2}$ film and the $\mathrm{SrTiO}_{3}$ substrate. Small changes in peak shapes of $\mathrm{O}$ 1s and $\mathrm{Sr} 3 \mathrm{~d}$ were possibly caused by these substrate contributions. Moreover, in the $\mathrm{O} 1 \mathrm{~s}$ spectrum, near $532 \mathrm{eV}$, a low intensity peak related to surface contaminants was observed in all films. A subtle change was observed in the $\mathrm{Cu} 2 \mathrm{p}$ spectrum near the main peak at $937 \mathrm{eV}$, as well as in the structure near 

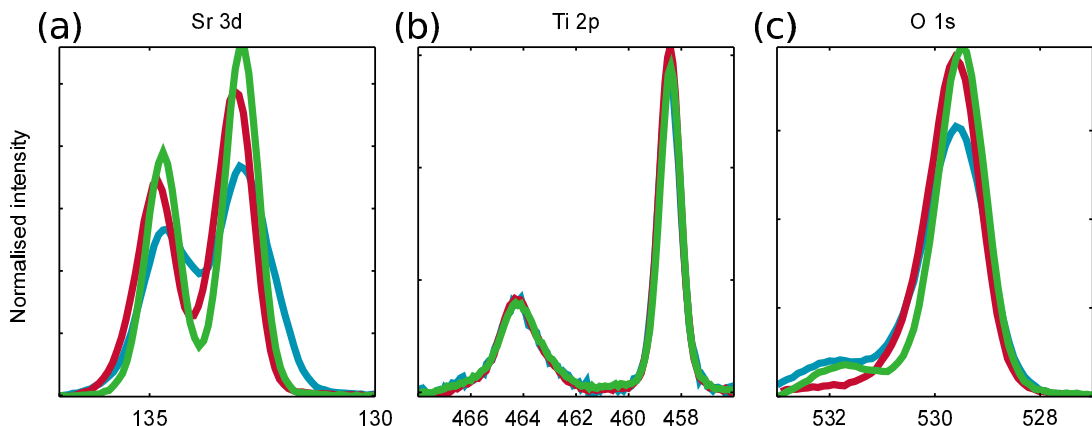

(d)

(e)

UPS
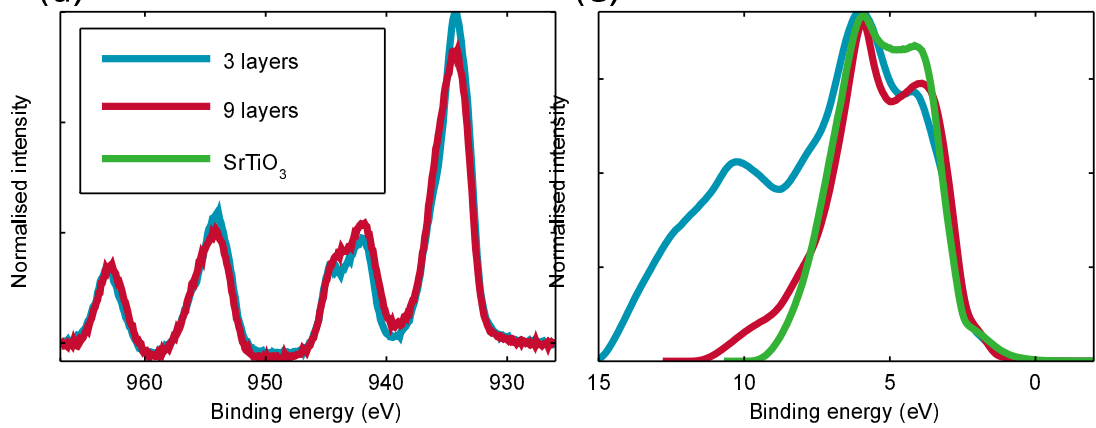

Figure 6.8: Photo-emission spectra of $\mathrm{SrCuO}_{2}$ core levels of (a) Sr 3d, (b) Ti 2p (c) O 1s, (d) $\mathrm{Cu} 2 \mathrm{p}$ and (e) valance band (UPS). For all XPS scans a Shirley background was subtracted and the intensity normalized to the total area, the Ti $2 p$ spectra were corrected for binding energy shifts due to sample charging. For UPS this charging was corrected by overlapping the main peak near $6 \mathrm{eV}$.

$942 \mathrm{eV}$. These changes were possibly caused by a change in coordination number or bond distance ${ }^{[131,133,134]}$. However, these were hard to quantify and beyond the scope of this work. The observed $\mathrm{Cu} 2 \mathrm{p}$ spectral shape with strong satellites on $2 p_{3 / 2}$ and $2 p_{1 / 2}$ at $943 \mathrm{eV}$ and $963 \mathrm{eV}$, respectively, indicated a dominant $\mathrm{Cu}^{2+}$ valence state in both of our $\mathrm{SrCuO}_{2}$ films

UPS spectra are shown in Fig. 6.8(e). Due to sample charging only qualitative comparison could be made. ${ }^{2}$ These results indicated the valance band of a three layer $\mathrm{SrCuO}_{2}$ film to be comparable to that of the $\mathrm{SrTiO}_{3}$ substrate. A shift in spectral weight was observed, but the general spectral shape remained constant. For the 9 layer $\mathrm{SrCuO}_{2}$ film additional peaks appeared at higher binding energy. This indicated a change in electronic structure.

\footnotetext{
${ }^{2}$ The spectra were shifted in energy in such a way that the main peak around $6 \mathrm{eV}$ overlaped for both $\mathrm{SrCuO}_{2}$ films and a $\mathrm{Nb}$ doped $\mathrm{SrTiO}_{3}$ substrate.
} 


\subsubsection{Quantitative photoemission}

To study if the observed structural transition is purely caused by structural rearrangement, quantitative XPS analysis was performed. The results are given in Table 6.1 based on the XPS data shown in Fig. 6.8. The percentages were calculated using calculated Scofield photoelectric cross-sections ${ }^{[135]}$ for the relative sensitivity factors. ${ }^{3}$ The Scofield cross-sections do not take into account machine specific corrections. Nonetheless, they yield reasonable results for an $\mathrm{SrTiO}_{3} \mathrm{sub}$ strate, also given in Table 6.1. In brackets the measured relative intensities of $\mathrm{Sr} 3 \mathrm{~d}, \mathrm{Cu} 2 \mathrm{p}, \mathrm{Ti} 2 \mathrm{p}$ and $\mathrm{O} 1 \mathrm{~s}$ are given. A Ti $2 \mathrm{p}$ signal of different magnitudes was clearly observed for both 3 and 9 unit-cell $\mathrm{SrCuO}_{2}$ films, with this being due to the greater inelastic attenuation of the $\mathrm{SrTiO}_{3}$ signal by the thicker film. Thus, the relative intensities of $\mathrm{Sr}, \mathrm{Cu}$ and $\mathrm{O}$ represented a combination of the $\mathrm{SrCuO}_{2}$ film and the underlying $\mathrm{SrTiO}_{3}$ substrate. Therefore, a correction was applied, whereby the observed Ti signal was subtracted from the $\mathrm{Sr}$ and $\mathrm{O}$ signals, assuming a stoichiometric $\mathrm{SrTiO}_{3}$ contribution. The resulting $\mathrm{SrCuO}_{2}$ compositions are shown without brackets in Table 6.1. This correction assumed a homogeneous $\mathrm{SrCuO}_{2}$ film and no dependence of the kinetic energy on the mean free path of the escaping electrons. The later assumption increased the experimental error, estimated at around 5\%. Taking into account the data analysis methodology, it was concluded that the two $\mathrm{SrCuO}_{2}$ films had a similar stoichiometry within the experimental error.

\subsection{Discussion}

SrCuO films on $\mathrm{TiO}_{2}$ terminated $\mathrm{Nb}$ doped $\mathrm{SrTiO}_{3}$ substrates had a RMS roughness of less than $0.4 \mathrm{~nm}$ and two-dimensional spotty RHEED patterns, both indicating atomically smooth surfaces. However, the surface is slightly roughened when the thickness is increased to 9 unit-cell layers compared to the 3 unit-cell film. Similar behavior was found by Samal et al. ${ }^{[128]}$ in superlattice heterostructures for thicker $\mathrm{SrCuO}_{2}$ layers. Since the observed roughness was smaller than the unit-cell height of $\mathrm{SrCuO}_{2}$, the effective thickness was not affected. No effects of this small roughness increase were found to give rise to a change in XPD or XPS signal.

Structural analyses using in situ XPD on an $\mathrm{Nb}$ doped $\mathrm{SrTiO}_{3}$ substrate showed good agreement with literature reports, for example by Pancotti et al. ${ }^{[120]}$. Moreover, forward scattering analysis predicts the main peaks at experimentally observed positions, near $45^{\circ}$ for the main peaks. Full MS simulations (EDAC) give a qualitative match with experimental results on $\mathrm{SrTiO}_{3}$ substrates. While, the main features in the simulated diffraction pattern are also visible in the experimental data, the relative intensities differ. This might be caused by the back-

\footnotetext{
${ }^{3}$ Quantitative XPS analysis was done using theoretical cross-sections, without taking into account the energy dependent inelastic losses and assuming a homogeneous film. This method allowed for comparing the changes in the stoichiometry of $\mathrm{SrCuO}_{2}$ films with respect to each other. However a more accurate estimation requires the consideration of inelastic losses.
} 
Table 6.1: XPD peak positions and corresponding c-axis lengths based on a forwards scattering approximation on the left side of the table. The right side indicates XPS relative intensities for $\mathrm{Sr} 3 \mathrm{~d}, \mathrm{Cu} 2 \mathrm{p}, \mathrm{O}$ 1s and Ti 2p high resolution spectra. A 20\%, 20\%, 60\% distribution was expected for stoichiometric $\mathrm{SrTiO}_{3}$ and $25 \%, 25 \%, 50 \%$ for $\mathrm{SrCuO}_{2}$. A Shirley background was removed and relative sensitivity factors were taken in to account. The substrate contributions to the spectral weight of $\mathrm{Sr}$ and $\mathrm{O}$ were removed by assuming a stoichiometric contribution of $\mathrm{SrTiO}_{3}$ based on the Ti peak. Relative intensities without this correction are shown in brackets.

\begin{tabular}{|c|c|c|c|c|c|c|c|}
\hline & \multicolumn{3}{|c|}{$\theta$ peak position \& c-axis } & \multicolumn{4}{|c|}{ Relative XPS intensity $( \pm 5 \%)$} \\
\hline & Sr 3d & $\mathrm{Cu} 3 \mathrm{p} / \mathrm{Ti} 2 \mathrm{p}$ & $\mathrm{O} 1 \mathrm{~s}$ & Sr 3d & $\mathrm{Cu} 2 \mathrm{p}$ & $\mathrm{O} 1 \mathrm{~s}$ & Ti $2 p$ \\
\hline 3 unit-cells & $46.8^{\circ} \pm 1^{\circ}$ & $45.0^{\circ} \pm 1^{\circ}$ & $45.4^{\circ} \pm 1^{\circ}$ & $30 \%$ & $23 \%$ & $47 \%$ & $0 \%$ \\
\hline $\mathrm{SrCuO}_{2}$ & $3.7 \pm 0.1 \AA$ & $3.9 \pm 0.1 \AA$ & $3.8 \pm 0.1 \AA$ & $(24 \%)$ & $(11 \%)$ & $(54 \%)$ & $(11 \%)$ \\
\hline 9 unit-cells & $49.0^{\circ} \pm 1^{\circ}$ & $49.9^{\circ} \pm 1^{\circ}$ & $49.9^{\circ} \pm 1^{\circ}$ & $27 \%$ & $25 \%$ & $48 \%$ & $0 \%$ \\
\hline $\mathrm{SrCuO}_{2}$ & $3.4 \pm 0.1 \AA$ & $3.3 \pm 0.1 \AA$ & $3.3 \pm 0.1 \AA$ & $(26 \%)$ & $(20 \%)$ & $(50 \%)$ & $(4 \%)$ \\
\hline $\mathrm{SrTiO}_{3}$ & $46^{\circ} \pm 1^{\circ}$ & $45^{\circ} \pm 1^{\circ}$ & $45^{\circ} \pm 1^{\circ}$ & $25 \%$ & $0 \%$ & $58 \%$ & $18 \%$ \\
\hline
\end{tabular}

ground removal as a function of $\theta$ which is applied to the experimental data. This effect is most pronounced for small $\theta$ angles. The machine specific background is approximated by a linear relationship and this is not valid for all $\theta$ values, as discussed in section 2.3.4. The effect of the background removal on the peak positions is small compared to the width of most XPD peaks. Therefore, the peak positions between $\sim 20^{\circ}$ and $\sim 70^{\circ}$ can still be compared qualitatively between experiment, forward scattering and simulations.

Photoelectron diffraction measurements of ultra-thin $\mathrm{SrCuO}_{2}$ films of 3 and 9 layers showed a clear shift in the main peak [101] position for all spectral lines. For $\mathrm{Cu} 3 \mathrm{p}$ and $\mathrm{O} 1 \mathrm{~s}$ the observed shifts were similar. However for Sr 3d the relative change between 3 and 9 unit-cell films was reduced. This reduction might be caused by a contribution of the $\mathrm{SrTiO}_{3}$ substrate to the $\mathrm{Sr} 3 \mathrm{~d}$ intensity from $\mathrm{SrCuO}_{2}$, lowering the observed $\theta$ position of the 9 unit-cell film. However, based on quantitative XPS analysis, an estimated maximum contribution of $11 \%$ for normal emission of $\mathrm{Sr}$ is assumed. Therefore, the XPD signal measured between 40 and 50 degrees was not significantly affected by the substrate. If this shift would be caused by Sr signal from the substrate, a similar shift should be observed in the $\mathrm{O}$ 1s signal. The $\mathrm{Sr} 3 \mathrm{~d}$ peak position of the $\mathrm{SrTiO}_{3}$ substrate was higher $\left(46^{\circ}\right)$ compared to the Ti $2 \mathrm{p}$ and $\mathrm{O} 1$ s peak position $\left(45^{\circ}\right)$ of the substrate. This small deviation in the $\mathrm{Sr} 3 \mathrm{~d}$ peak position was found in $\mathrm{SrTiO}_{3}$ and $\mathrm{SrCuO}_{2}$ ultra-thin films and thus likely arises from an intrinsic diffraction effect for $\mathrm{Sr} 3 \mathrm{~d}$ electrons in perovskite like unit-cells. This statement is supported by the MS simulations, 
which also show a smaller $\theta$ shift for Sr emission as compared to $\mathrm{O} 1 \mathrm{~s}$ emission.

The mismatch observed between MS simulations and experiment for $\mathrm{Cu} 3 \mathrm{p}$ photoelectrons, especially for the 3 unit-cell film is not well understood. The experimental signal to noise ratio is low, hampering qualitative analyses apart from observing a main peak near $45^{\circ}$. The MS simulation of a chain-type film does not show a peak near $45^{\circ}$ as one would expect from forward scattering principles. Why this peak is not observed in simulations in not clear. The reduced in-plane symmetry has a strong effect on the simulated diffraction pattern (not shown). Such asymmetries are not experimentally observed, as shown in Fig. 6.5. This discrepancy requires a more detailed study of the simulation parameters, e.g., using larger clusters. The simulations do qualitatively predict the flat and featureless $\mathrm{Cu}$ angular emission distribution for chain-type $\mathrm{SrCuO}_{2}$.

Using a forward scattering analysis of the measured main peak $\theta$ angles, caxis parameters of both $\mathrm{SrCuO}_{2}$ films are extracted. Experimentally a c-axis of $3.35 \pm 0.1 \AA$ is found for a 9 layer thick $\mathrm{SrCuO}_{2}$ film and a c-axis of $3.80 \pm 0.1 \AA$ is found for a 3 layer $\mathrm{SrCuO}_{2}$ film. This is in good agreement of the theoretically predicted structural phase transition. ${ }^{[114]}$

Using quantitative XPS, both $\mathrm{SrCuO}_{2}$ films are found to have a similar stoichiometry. Due to the use of theoretical cross-sections the error-margin in the absolute stoichiometry values is large, making statements about the absolute stoichiometry weak. However, the films can be compared to each other and a similar analysis of an $\mathrm{SrTiO}_{3}$ substrate using Scofield cross-sections yields reasonable result. Although limited, the analysis performed here indicates that the structural rearrangement takes place without a change in film stoichiometry.

In both XPS and UPS experimental results, changes in the peak shape are observed when increasing the $\mathrm{SrCuO}_{2}$ film thickness from 3 to 9 unit-cell. This indicates a change in electronic structure as also discussed by Samal et al. ${ }^{[128]}$. More detailed analyses and further study using photoemission is of high interest for future developments, i.e., tuning the electronic properties. However, this electronic analysis is beyond the scope of this work.

\subsection{Conclusions}

A structural phase transition in $\mathrm{SrCuO}_{2}$ ultra-thin single-layer films as a function of film thickness is demonstrated using XPD. A film of 3 unit-cells thick is confirmed to be of the chain-type and a 9 unit-cells thick films is of the planar type. Using both simple forward focusing arguments and MS simulations, the measured peak positions are found to be in accordance with the predicted structure. The observed structural change occurs while the stoichiometry is conserved, as observed by photoelectron spectroscopy. The present findings provide new insight for designing artificial cuprate heterostructures with new electronic properties. 


\title{
Chapter 7
}

\section{Role of interfacial oxygen octa- hedral coupling in strained $\mathrm{SrRuO}_{3}$ films}

\begin{abstract}
By applying epitaxial strain to ultra-thin films of ferromagnetic $\mathrm{SrRuO}_{3}$, one can essentially tune its structure and properties. Substrate induced strain is commonly used to alter the physical properties of $\mathrm{SrRuO}_{3}$, effectively by adjusting the structural parameters, i.e., bond distances and $\mathrm{RuO}_{6}$ octahedral tilts and rotations. In this chapter, the role of oxygen octahedral coupling across the substrate-film and film-capping layer interfaces on the magnetic properties of $\mathrm{SrRuO}_{3}$ is studied in detail. This interfacial coupling is shown to provide a new way to control the properties of $\mathrm{SrRuO}_{3}$, in addition to merely considering substrate induced strain. Experimental evidence for an enhancement of the ferromagnetic Currie temperature of $\mathrm{SrRuO}_{3}$ by the addition of a $\mathrm{SrTiO}_{3}$ capping layer is provided, which is driven by octahedral coupling. The model is supported by density functional calculations. Moreover, uncapped ultrathin $\mathrm{SrRuO}_{3}$ films show a thickness dependent metal-insulator transition which is not well-understood. The role of octahedral coupling on this metal-insulator transition is explored. Initial experimental results are provided as well as DFT calculation.
\end{abstract}

Part of the work presented in this chapter is prepared as a journal article: S. Thomas, B. Kuiper, J. Hu, Z. Zhong, R. Wu, G. Rijnders, G. Koster, and J. Xia. Capping-layer-induced enhancement of ferromagnetism in ultra-thin $\mathrm{SrRuO}_{3}$ films. $(2013)^{[136]}$ 


\subsection{Introduction}

Complex oxide thin films heterostructures and interfaces have recently attracted great attention. By inducing strain one can essentially tune the structure and properties of such thin films to reveal new or enhanced physical properties, often very different from bulk. For example, enhancement of ferroelectricity by using substrate induced strain has been demonstrated in thin films of $\mathrm{SrTiO}_{3}{ }^{[12]}$ and $\mathrm{BaTiO}_{3}{ }^{[137]}$. Moreover, ferromagnetism in $\mathrm{SrRuO}_{3}{ }^{[100,138]}$ has been tuned by substrate induced strain.

Nowadays, the role of oxygen octahedral rotations in perovskite-type thin films and interfaces gets more attention in this regard. Next to strain induced structural changes, coupling of the octahedral network due to corner connectivity at oxide interfaces is postulate to significantly influence film properties. ${ }^{[13,139]}$ Octahedral distortions, i.e., rotations and tilts are postulated to extend several layers across interfaces, like observed in $\mathrm{SrRuO}_{3}$ thin films on $\mathrm{GdScO}_{3}$ substrates ${ }^{[14]}$. Essentially, one might make use of the octahedral coupling across interfaces, independent of the substrate induced lattice strain, to tune film properties by careful selection of the substrates lattice constant and octahedral tilt pattern. ${ }^{1}$

$\mathrm{SrRuO}_{3}$ is an interesting member of the perovskite family as it is one of the few undoped conducting complex oxides and therefore commonly used as an electrode layer in complex oxide heterostructures. From a fundamental point of few $\mathrm{SrRuO}_{3}$ is also widely studied due to its interesting physical properties, e.g., bad metal behavior at high temperature, $4 \mathrm{~d}$ itinerant ferromagnetism, intermediate electron correlation and large magnetocrystalline anisotropy. ${ }^{[100]}$ The itinerant ferromagnetism allows for the possible use of $\mathrm{SrRuO}_{3}$ in the field of spintronics. ${ }^{[140,141]}$. A critical thickness for the onset of ferromagnetism is found near 4 unit-cell (u.c.) layers in thickness; below 4 u.c. no conductivity and ferromagnetism is observed. However, for films above 4 u.c. in thickness, the complete film is conducting and magnetic. No dead layer is observed and all layers are found to contribute to the magnetic signal. ${ }^{[142]}$ The origin of this transition is not well-understood. In a recent report the critical thickness is found the be reduced when creating artificial stacks or superlattice structures which contain $\mathrm{SrRuO}_{3}$ layers with a thickness below 4 unit-cell layers. ${ }^{[143]}$

The ferromagnetic Currie temperature $\left(\mathrm{T}_{\mathrm{C}}\right)$ of $\mathrm{SrRuO}_{3}$ in strained thin films, depends on the film thickness. A $\mathrm{T}_{\mathrm{C}}$ of $155 \mathrm{~K}$ is observed for thick films, which is near the bulk value. However, upon reducing the thickness down to the critical limit, a $\mathrm{T}_{\mathrm{C}}$ below $120 \mathrm{~K}$ is found. ${ }^{[100,142]}$ An enhancement of $\mathrm{T}_{\mathrm{C}}$ is found for $\mathrm{SrRuO}_{3}$ films on $\mathrm{SrTiO}_{3}$ after removal of the $\mathrm{SrTiO}_{3}$ substrate by chemical etching. ${ }^{[138]}$ The $\mathrm{T}_{\mathrm{C}}$ of $\mathrm{SrRuO}_{3}$ is highly sensitive measure for the amount of induced strain or structural deformation in thin films.

In this chapter, the effects of reduced film thickness and octahedral coupling are studied in epitaxial ultra-thin $\mathrm{SrRuO}_{3}$ films on $\mathrm{SrTiO}_{3}(001)$ and $\mathrm{DyScO}_{3}(110)$

\footnotetext{
${ }^{1}$ Note that the octahedral tilt patterns in bulk perovskite-type materials can by described using Glazer notation. ${ }^{[24]}$ These tilt patterns each correspond to a specific crystal structure (space group).
} 
(a)

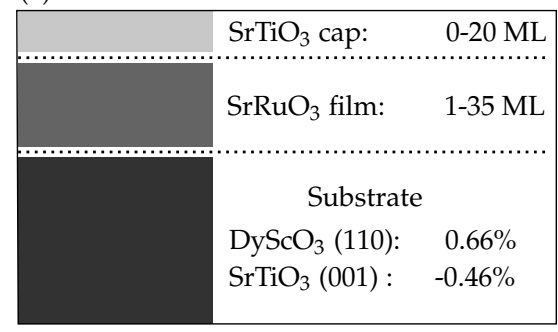

(b)

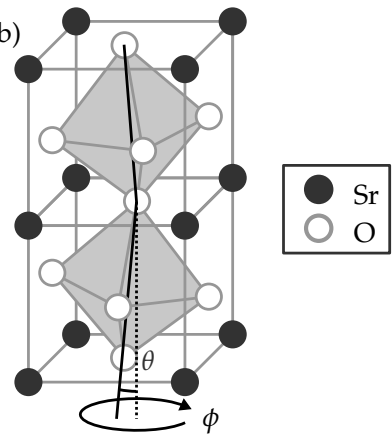

Figure 7.1: Schematic (a) of the used sample geometries of thin film structures discussed in this chapter. $\mathrm{SrRuO}_{3}(110)$ films are grown on $\mathrm{DyScO}_{3}(110)$ and $\mathrm{SrTiO}_{3}$ (001) single crystal substrates in the specified thickness ranges. Schematic (b) of the octahedral inplane rotations $(\phi)$ and out-of-plane tilts $(\theta)$ of in perovskite oxides. For clarity, only two psuedo-cubic blocks are drawn in this schematic instead of the full orthorhombic unit-cell of $\mathrm{SrRuO}_{3}$.

substrates. An enhancement of $\mathrm{T}_{\mathrm{C}}$ is found for $\mathrm{SrRuO}_{3}$ films on $\mathrm{DyScO}_{3}$ substrates capped with several layers of $\mathrm{SrTiO}_{3}$. The $\mathrm{T}_{\mathrm{C}}$ is studied by using a loopless Sagnac interferometer ${ }^{[144]}$, measuring the polar magneto-optic Kerr effect (MOKE). The observed $\mathrm{T}_{\mathrm{C}}$ enhancement is related to a change in octahedral tilt patterns induced by the capping-layer and supported by density functional theory (DFT) calculations. Especially under tensile strain, $\mathrm{SrRuO}_{3}$ is found to be highly sensitive to the addition of a capping layer.

Moreover, DFT calculations predict a possible structural transformation in (uncapped) ultra-thin $\mathrm{SrRuO}_{3}$ films where the octahedral tilt patterns are altered and a possible anti-ferromagnetic ground state of this structural phase is postulated to cause the observed critical thickness behavior. Using both structural and magnetic characterization techniques an attempt is made to confirm this predicted phase transition.

In this chapter, first the sample geometries used to study $\mathrm{SrRuO}_{3}$ are discussed along with the structural properties of $\mathrm{SrRuO}_{3}$. Next, DFT calculation results are discussed which predict a tetragonal ground state of $\mathrm{SrRuO}_{3}$, for which initial experimental evidence is provided by XRD and magnetic dichroism. Finally, the enhancement of $T_{C}$ by addition of a capping layer is shown and DFT models which support these findings are discussed.

\subsubsection{Sample geometry, orientation and structure}

Epitaxial $\mathrm{SrRuO}_{3}$ films prepared using pulsed laser deposition (PLD) with various thickness, strain and capping layer thickness are discussed here. $\mathrm{SrRuO}_{3}$ has an orthorhombic crystal structure, isostructural with $\mathrm{GdFeO}_{3}(\mathrm{a}=5.5670 \AA, \mathrm{b}=$ $5.5304 \AA$ and $c=7.8446 \AA)^{[145]}$ Its structure can be represented as a pseudo-cubic structure with a lattice parameter of $\mathrm{a}_{p c}=3.923 \AA$. The $\mathrm{RuO}_{6}$ octahedra show 
out-of-phase rotations about the $[\overline{1} 10]_{\text {ortho }}$ and in-phase around the $[001]_{\text {ortho }}$. directions, glazer notation: $\mathrm{a}^{-} \mathrm{a}^{-} \mathrm{c}^{+} \cdot{ }^{[14]}$ At temperatures above $550{ }^{\circ} \mathrm{C}$ a tetragonal phase is stabilized with out-of-phase rotations around the [001] $]_{\text {ortho }}$ and above $680{ }^{\circ} \mathrm{C}$ a cubic phase is formed in bulk $\mathrm{SrRuO}_{3} \cdot{ }^{[146]}$ Films grown on $\mathrm{SrTiO}_{3}(100)$ and $\mathrm{DyScO}_{3}$ (110) substrates grow in the (110) ortho. direction; i.e., the orthorhombic [001] direction lies in-plane with respect to the film surface. $\mathrm{SrTiO}_{3}$ has a lattice constant of $3.905 \AA$ resulting in compressively strained $\mathrm{SrRuO}_{3}$ films $(-0.46 \%)$ and $\mathrm{DyScO}_{3}$ has a pseudo-cubic lattice constant of $3.949 \AA(0.66 \%){ }^{2}$

Films of $\mathrm{SrRuO}_{3}$ with a thickness of 1 to 35 psuedo-cubic unit-cell layers $(\sim 14 \mathrm{~nm})$ are prepared on both $\mathrm{SrTiO}_{3}(001)$ and $\mathrm{DyScO}_{3}(110)$ substrates with a $\mathrm{SrTiO}_{3}$ capping layer thickness between 0 and 20 u.c. A schematic of the sample geometries is shown in Fig. 7.1(a). The tilt and rotation angles, $\theta$ and $\phi$ discussed in this chapter are shown in the schematic in Fig. 7.1(b).

\subsection{Critical thickness of strained $\mathrm{SrRuO}_{3}$}

Ultra-thin films of $\mathrm{SrRuO}_{3}$ show a critical thickness for the onset of ferromagnetism and conductivity near 4 u.c. layers, as shown by Xia et al. ${ }^{[142]}$. However, Chang et al. ${ }^{[147]}$ report a critical thickness of 2 u.c. layers. Chang et al. ${ }^{[147]}$ argue that the discrepancy between their results and the work of Xia et al. ${ }^{[142]}$ is related to different growth speeds. High growth speeds could result in disordered film growth, leading to decreased conductivity and magnetism. However, the surface morphologies of 2 u.c. thick films in both reports appear similar. The reports use different laser fluence values and the thickness calibration is done using different methods in both cases. Nonetheless, in both reports a metal-insulator transition is observed, which is not well-understood. Recently, Liu et al. ${ }^{[143]}$ also found ferromagnetism in 2 u.c. layer $\mathrm{SrRuO}_{3}$ films, when grown in a superlattice structure combined with $\mathrm{LaAlO}_{3}$. Below results from DFT calculations on the structure and magnetic state of $\mathrm{SrRuO}_{3}$ are discussed for strained single-layer $\mathrm{SrRuO}_{3}$ films with thickness of a few unit-cell layers. Experimental evidence which partially agrees with the predictions is acquired using XRD and XMCD/XMLD and is given at the end of this section.

\subsubsection{Ground state of tetragonal $\mathrm{SrRuO}_{3}$}

Results of DFT calculations are depicted in Fig. 7.2 using the LDA+U approximation ${ }^{3}$, for all three bulk structures of $\mathrm{SrRuO}_{3}$. The structural energies of the tetragonal an cubic structures were found to be roughly 30 and $200 \mathrm{meV} / \mathrm{Ru}$ higher compared to the bulk orthorhombic structure, which is consistent with the observed structural transitions of $\mathrm{SrRuO}_{3}$ from orthorhombic to tetragonal to

\footnotetext{
${ }^{2}$ Strain is calculated using: $\left(a_{s}-a_{f}\right) / a_{f}$ where $a_{s}$ and $a_{f}$ are the pseudo-cubic lattice constants of the substrate and film respectively.

${ }^{3}$ DFT calculations using the GGA approximation gave qualitatively consistent results.
} 

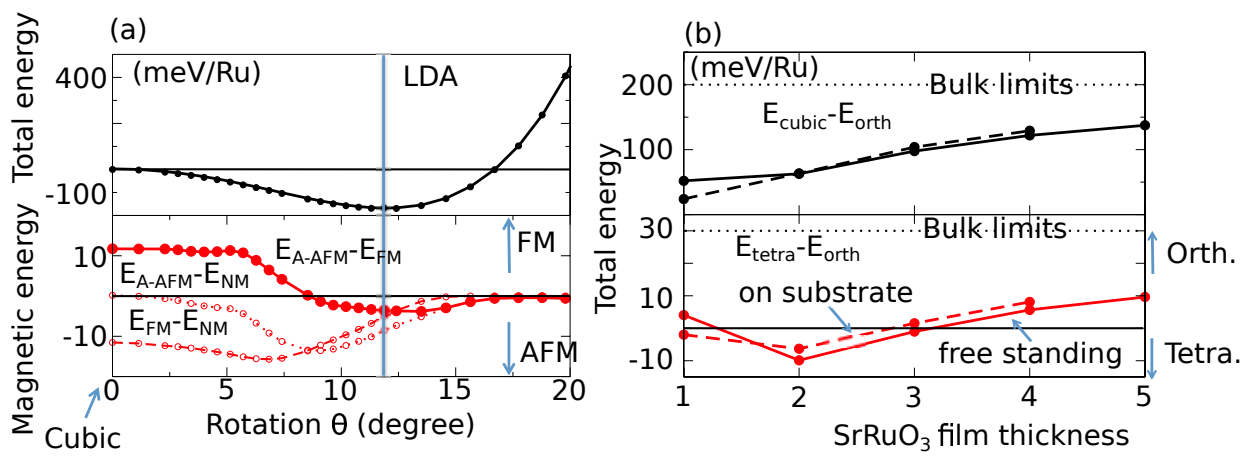

(c)

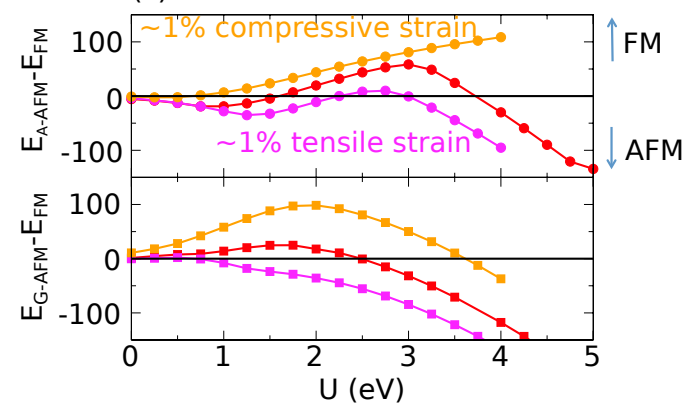

Figure 7.2: DFT calculation results of $\mathrm{SrRuO}_{3}$. In (a) the magnetic ground state of a tetragonal $\mathrm{SrRuO}_{3}$ phase is studied by comparing the total and magnetic energy of various magnetic configurations as function of octahedral rotation angle. Here the vertical line depicts the energy minimized tilt angle and a tilt of zero degree corresponds to a cubic structure. In (b) the structural phases of $\mathrm{SrRuO}_{3}$ on $\mathrm{SrTiO}_{3}$ are compared as a function of film thickness; the dotted lines are calculated by fixing the in-plane lattice parameters; for the solid lines also the octahedral coupling with $\mathrm{SrTiO}_{3}$ is taken into account. In (c) the magnetic energy difference between possible AFM and FM phases for tetragonal $\mathrm{SrRuO}_{3}$ are calculated as function of the correlation term, U. The DFT calculations were performed by Zhicheng Zhong, see also section 2.4.2

cubic as a function of temperature. ${ }^{[146]}$ In Fig. 7.2(a) the total energy and magnetic energies of possible magnetic ordering in tetragonal $\mathrm{SrRuO}_{3}$ is shown as a function of the octahedral rotation angle. At the energy minimized tilt angle $(\sim$ $\left.12^{\circ}\right)$ the A-type anti-ferromagnetic $(\mathrm{A}-\mathrm{AFM})^{4}$ phase is preferred over the ferromagnetic (FM) phase and none magnetic (NM) phase. This indicates the ground state of $\mathrm{SrRuO}_{3}$ with a tetragonal structure is possibly AFM instead of FM.

Interestingly, as discussed above, substrate induced strain might influence the structure of $\mathrm{SrRuO}_{3}$, especially in ultra-thin epitaxial films. Therefore, the total energy of orthorhombic and tetragonal $\mathrm{SrRuO}_{3}$ were calculated strained on $\mathrm{SrTiO}_{3}$. The in-plane lattice parameters were fixed and/or at the interface

\footnotetext{
${ }^{4}$ In an A-type anti-ferromagnetic structure anti-ferromagnetic coupling occurs between layers and ferromagnetic coupling occurs within one layer. ${ }^{[148]}$
} 
the octahedral network was connected. Calculation results of the total energy of $E_{\text {cubic }}-E_{\text {orth }}$ and $E_{\text {tetra }}-E_{\text {orth }}$ are shown in Fig. 7.2(b) as a function of film thickness. The dotted lines were calculated by fixing the strain and substrate interface; the solid lines were calculated by only fixing the in-plane lattice constant. The orthorhombic phase was favored over the cubic phase independent of film thickness. However, the tetragonal phase was favored for films below 3 u.c., especially for $\mathrm{SrRuO}_{3}$ films fixed on a $\mathrm{SrTiO}_{3}$ substrate. Indicating a possible tetragonal structure can be obtained in ultra-thin $\mathrm{SrRuO}_{3}$ films on $\mathrm{SrTiO}_{3}$.

In Fig. 7.2(c) the effects of substrate induced strain were calculated by comparing $A$ and $G$ type $^{5}$ AFM phases to the FM phase for different values of $U$, since $U$ was not well-known for $\mathrm{SrRuO}_{3}$. Tensile strain of $1 \%$ favors both types of AFM phases, while compressive strain of $1 \%$ favors the FM phase for most values of $U$.

These calculations indicate that it is possible that strain induces a tetragonal structure in $\mathrm{SrRuO}_{3}$ thin films below 3 u.c. This tetragonal structure has an antiferromagnetic ground state. This agrees with previous experimental observations of a critical thickness in $\mathrm{SrRuO}_{3}$ and indications of exchange bias coupling. ${ }^{[142]}$

\subsubsection{Increased symmetry in ultra-thin films}

To experimentally verify the possible formation of a tetragonal ground state in ultra-thin $\mathrm{SrRuO}_{3}$ films on $\mathrm{SrTiO}_{3}$, as predicted above, XRD experiment were done. To be able to study films of only several nanometers in thickness a synchrotron X-ray source was used, see also section 2.3.2. Here, the crystal directions were defined in pseudo-cubic notation, where the [001] direction is out-of-plane. Thus an L-scan was done along the $[110]_{\text {ortho }}$ direction.

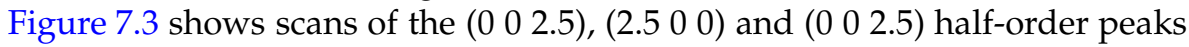
of a 2 and 8 u.c. $\mathrm{SrRuO}_{3}$ film on $\mathrm{SrTiO}_{3}$. In the L-scan shown in (a) of the $(00$ 2.5) peak, a clear peak was observed in the 8 u.c. sample near $\mathrm{L}=2.475$. Whereas, for the 2 u.c. sample this peak was not observed ${ }^{6}$ indicating a higher symmetry. H-scans (in-plane) of both films (b) and (c) did not show any peaks, suggesting that rotations were suppressed along orthogonal in-plane directions. Note that the $\mathrm{SrTiO}_{3}$ substrate peak near $\mathrm{L}=2.5$ was observed due to higher harmonics of double frequency in the synchrotron spectrum, which gives some signal on high quality crystalline materials.

The $\left(\begin{array}{ll}0 & 0\end{array} 2.5\right)$ reflection should be forbidden if octahedra rotate strictly around the [001] direction and was not observed in thick $\mathrm{SrRuO}_{3}$ films. Possibly the octahedra were deformed in the 8 u.c. film. When comparing the 2 and 8 u.c. films, the 2 u.c. film has a more regular octahedral tilt and/or rotation pattern compared to the 8 u.c. film.

\footnotetext{
${ }^{5}$ G-type antiferromagnetic order is obtained when anti-ferromagnetic ordering occurs both in between layers and within one layer. ${ }^{[148]}$

${ }^{6}$ The missing $\left(\begin{array}{lll}0 & 0 & 2.5\end{array}\right)$ peak in the 2 u.c. sample was expected not to be missing due to lack of intensity from the thin film.
} 


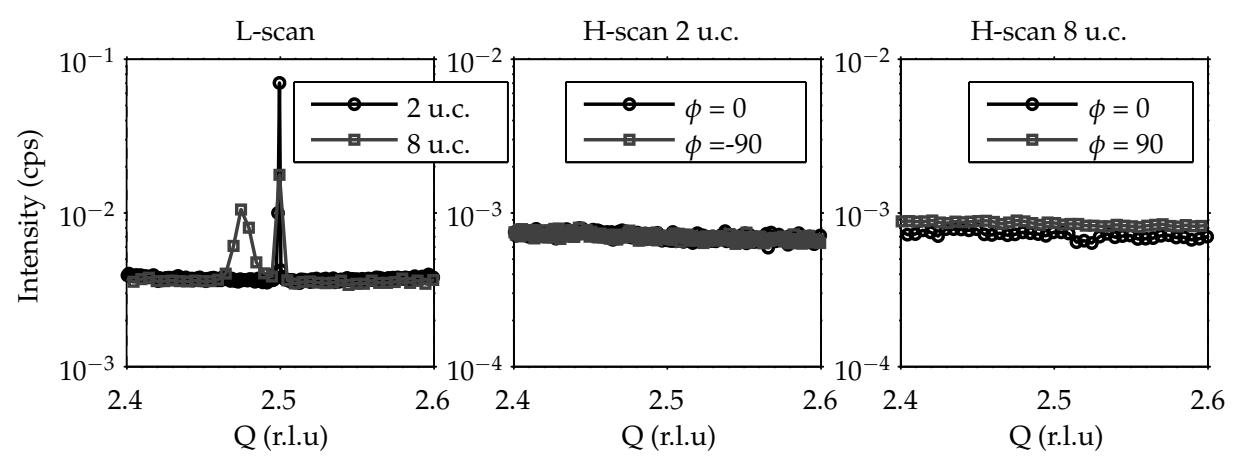

Figure 7.3: Synchrotron $\mathrm{XRD}$ on $\mathrm{SrRuO}_{3}$ films of 2 and 8 u.c. thick on $\mathrm{SrTiO}_{3}$. (a) Scans of the $\left(\begin{array}{ll}0 & 0 \\ 2.5\end{array}\right)$ reflection show a half-order peak in 8 u.c. sample, but not in the 2 u.c. sample. In-plane scans (b) and (c) show no half order peak. XRD results indicate the 2 u.c. sample has a higher symmetry compared to the 8 u.c. sample. Note that a sharp peak related to the $\mathrm{SrTiO}_{3}$ substrate at $\mathrm{L}=2.5$ is visible for both samples in (a).

\subsubsection{Search for a possible AFM phase}

X-ray magnetic dichroism experiments were done on two $\mathrm{SrRuO}_{3}$ films grown on $\mathrm{DyScO}_{3}$ substrates, one with a thickness of 3 u.c. and an thicker reference sample of $13 \mathrm{~nm}$. Using both circular and linear dichroism it is possible to determine possible ferromagnetic (FM) and anti-ferromagnetic (AFM) ordering. The $13 \mathrm{~nm}$ $\mathrm{SrRuO}_{3}$ film showed a clear XMCD signal as shown in Fig. 7.4(a), while the 3 u.c. sample showed a very reduced XMCD signal in the same field ( 2 Tesla). The strength of the XMCD signal of this 3 u.c. sample was studied as a function of the applied magnetic field in Fig. 7.4(b); a linear relationship is found which describes paramagnetic behavior. Moreover, no magnetic moment was measured at zero field and $5 \mathrm{~K}$, indicating the film is not ferromagnetic.

Linear dichroism experiments (XMLD) were also performed on both samples. These measurements can be used to understand if there is an AFM phase in the system. Assuming that, a spin ordering takes place which induces the spins to preferentially be aligned in-plane or out-of-plane. Only a very small XMLD signal was observed for both samples, as shown in Fig. 7.4(c). To eliminate the orbital effects from a possible magnetic contribution, the XMLD signal as defined as $\mathrm{XLD}_{10 \mathrm{~K}}-\mathrm{XLD}_{300 K}$, where XMLD was the magnetic part of the linear dichroism signal. The result was that there was no magnetic contribution to the XLD; no AFM phase was observed in both the $13 \mathrm{~nm}$ and 3 u.c. films down to $10 \mathrm{~K}$. Therefore, the 3 u.c. film was found to be paramagnetic and not to be antiferromagnetic and not ferromagnetic. 

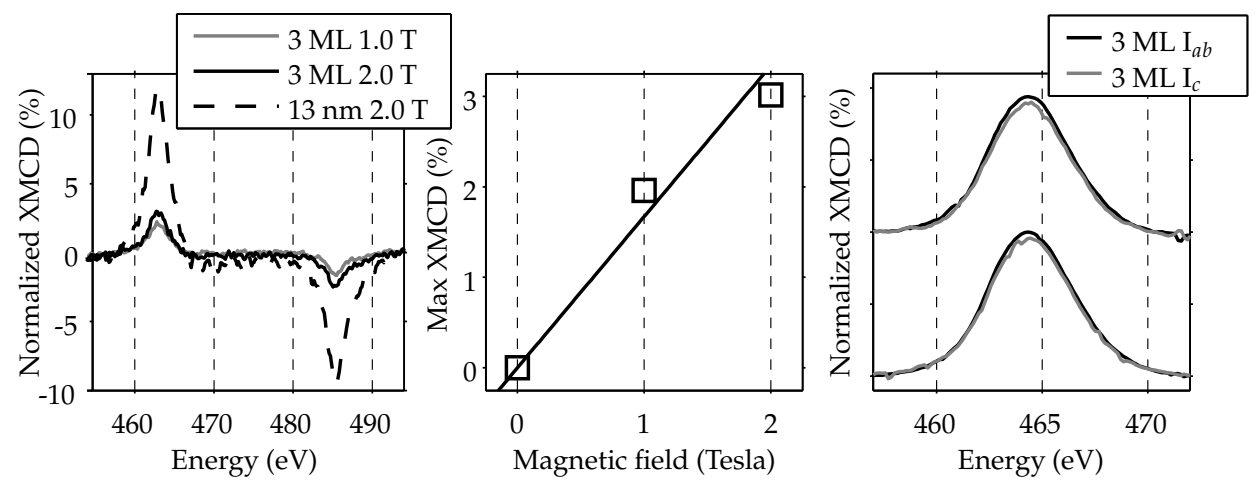

Figure 7.4: Xray magnetic linear and circular dichroism results of; (a) XMCD normalized intensity of the $\mathrm{Ru}$ M-edge for a 3 u.c. and a $13 \mathrm{~nm} \mathrm{SrRuO}_{3}$ film on $\mathrm{DyScO}_{3}$ without a capping layer showing a magnetic contribution from $\mathrm{Ru}$; (b) maximum of the XMCD $\mathrm{M}_{3}$ signal as a function of the magnetic field for the 3 u.c. sample; (c) XAS adsorption spectra for both samples for linear polarization of light parallel to the interface (black line) and perpendicular to the interface (red line).

\subsubsection{Structural characterization by XPD}

Structural analysis of ultra-thin films by XRD indicated a possible change in crystal structure when the $\mathrm{SrRuO}_{3}$ film thickness is reduced. Since the signal strength obtained from such thin layer is low, a surface sensitive technique like X-ray photoelectron diffraction (XPD) could provide an alternative way to study the structural properties of $\mathrm{SrRuO}_{3}$ ultra-thin films. Due to this surface sensitivity, XPD also allows for the structural study of films with less than a monolayer of coverage.

The structure of $20 \mathrm{~nm}$ films of $\mathrm{SrRuO}_{3}$ is studied on $\mathrm{DyScO}_{3}$ and $\mathrm{SrTiO}_{3}$. Since $\mathrm{SrRuO}_{3}$ is nearly tetragonal on $\mathrm{DyScO}_{3}$ and nearly orthorhombic on $\mathrm{SrTiO}_{3}$, a change in symmetry is expected. ${ }^{[79]} \mathrm{XPD}$ results of two $\sim 20 \mathrm{~nm} \mathrm{SrRuO} 3$ films grown on $\mathrm{SrTiO}_{3}$ and $\mathrm{DyScO}_{3}$ are shown in Fig. 7.5. In (a)-(c) stereographic projections of the intensity maps of the $\mathrm{Sr} 3 \mathrm{~d}, \mathrm{Ru} 3 \mathrm{p}$ and $\mathrm{O} 1 \mathrm{~s}$ emission lines are shown of $\mathrm{SrRuO}_{3}$ on $\mathrm{SrTiO}_{3}$. Clear four fold symmetry was observed in all spectra. However, $\mathrm{SrRuO}_{3}$ on $\mathrm{SrTiO}_{3}$ is known to be orthorhombic. Therefore, small variations in peak position are expected for different $\phi$ angles. High resolution $\theta$-scans of the pseudo-cubic [101], [011], [1101] and [0111] directions of $\mathrm{SrRuO}_{3}$ are shown in Fig. 7.5(d) and (e) on $\mathrm{DyScO}_{3}$ and $\mathrm{SrTiO}_{3}$ respectively.

The observed peak positions are given in Table 7.1. The values shown are averaged over all four $\phi$ angles and the standard deviations are indicated. The variations observed in the peak position on each sample were of the order of $0.2^{\circ}$, but did not show any systematic behavior, as was expected based in XRD result. Even larger variations were observed for an $\mathrm{SrTiO}_{3}$ substrate, depicted in Fig. $7.5(\mathrm{f}) . \theta$-shifts of less than $0.2^{\circ}$ in $\theta$ are expected for an orthorhombic to 

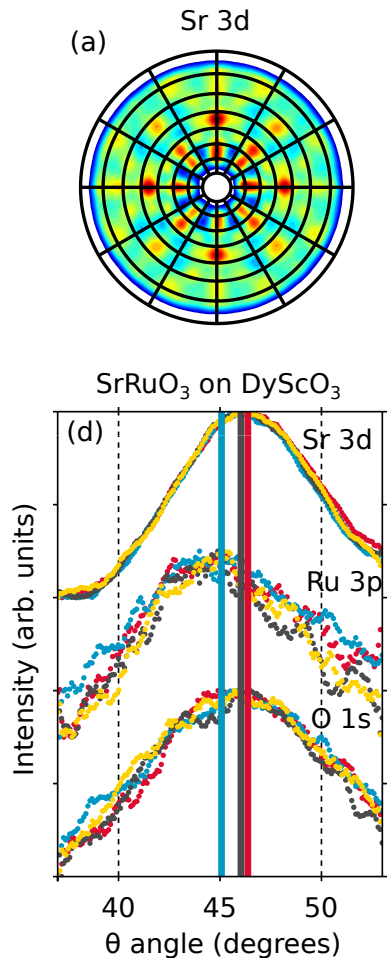

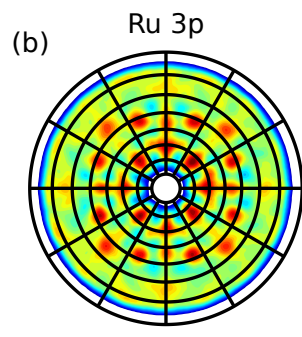

$\mathrm{SrRuO}_{3}$ on $\mathrm{SrTiO}_{3}$

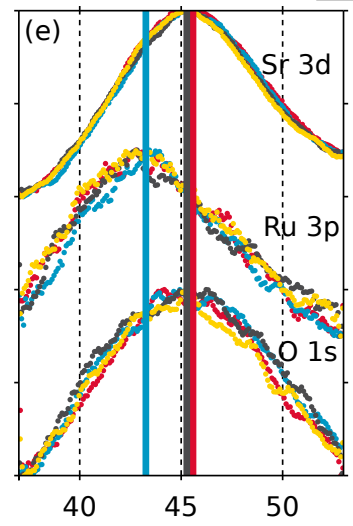

$\theta$ angle (degrees)
0 is

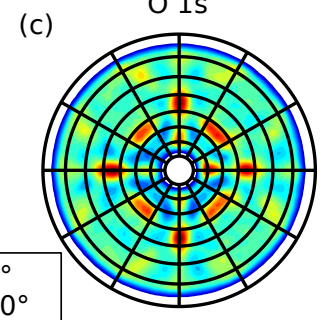

$\mathrm{SrTiO}_{3}$

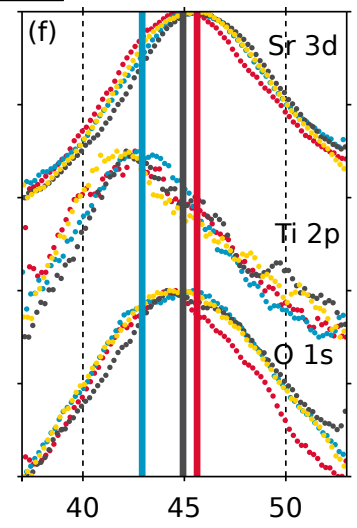

$\theta$ angle (degrees)

Figure 7.5: XPD results from $\mathrm{SrRuO}_{3}$ grown on $\mathrm{DyScO}_{3}$ and $\mathrm{SrTiO}_{3}$. (a) Sr 3d intensity map, (b) Ru 3p intensity map and (c) O 1s intensity map of a $20 \mathrm{~nm} \mathrm{SrRuO}_{3}$ film on $\mathrm{SrTiO}_{3}$, showing a four-fold symmetry. The black lines indicate $\theta$ steps of $10^{\circ}$ and $\phi$ steps of $30^{\circ}$. (d) The exact symmetry is studied by high resolution $\theta$ scans at different $\phi$ angles of the [101], [011], [1̄01] and [0111] peaks of $\mathrm{SrRuO}_{3}$ on $\mathrm{DyScO}_{3}$. (e) Similar $\theta$ scans of $\mathrm{SrRuO}_{3}$ on an $\mathrm{SrTiO}_{3}$ substrate. (f) $\theta$ scans of an $\mathrm{Nb}$ doped $\mathrm{SrTiO}_{3}$ substrate, here the Ti $2 \mathrm{p}$ is shown instead of the $\mathrm{Ru} 3 \mathrm{p}$. The observed average $\theta$ value are slightly reduced for $\mathrm{SrRuO}_{3}$ on $\mathrm{SrTiO}_{3}$ compared to $\mathrm{SrRuO}_{3}$ on $\mathrm{DyScO}_{3}$. The average $\theta$ peak position values are indicated by vertical lines: $\mathrm{Sr}$ (red), $\mathrm{Ru} / \mathrm{Ti}$ (blue) and $\mathrm{O}$ (gray). The measured $\theta$ values are given in Table 7.1.

tetragonal transition. The observed variations in the peak position for an $\mathrm{SrTiO}_{3}$ substrate were close to $0.2^{\circ}$, indicating the current XPD experiments are not able to distinguish the structural transition in thick $\mathrm{SrRuO}_{3}$ films. Therefore, it was not possible to determine the octahedral tilt patterns based on analysis of the main peaks in $\theta$-scans as a function of $\phi$ angle.

Although, the variations of $\theta$ positions as a function of $\phi$ do not provide the desired structural information, the average $\theta$ positions did vary with the applied strain. Since $\mathrm{SrRuO}_{3}$ is compressively strained on $\mathrm{SrTiO}_{3}$, the c-axis is in- 
Table 7.1: $\theta$-peak positions obtained using Gaussian fits of the experimental data plotted in Fig. 7.5. The observed peak positions and the standard deviation in the peak position for the different $\phi$ angles are given.

\begin{tabular}{rccc}
\hline \hline & $\mathrm{SrRuO}_{3}$ on $\mathrm{DyScO}_{3}$ & $\mathrm{SrRuO}_{3}$ on $\mathrm{SrTiO}_{3}$ & $\mathrm{SrTiO}_{3}$ substrate \\
\cline { 2 - 4 } $\mathrm{Sr} \mathrm{3d}$ & $46.4 \pm 0.1^{\circ}$ & $45.6 \pm 0.1^{\circ}$ & $45.6 \pm 0.3^{\circ}$ \\
$\mathrm{Ru}$ 3p $/ \mathrm{Ti}$ 2p & $45.1 \pm 0.2^{\circ}$ & $43.3 \pm 0.2^{\circ}$ & $42.9 \pm 0.3^{\circ}$ \\
$\mathrm{O}$ 1s & $46.0 \pm 0.2^{\circ}$ & $45.3 \pm 0.1^{\circ}$ & $44.9 \pm 0.3^{\circ}$ \\
\hline \hline
\end{tabular}

creased. Simple forward focusing analyses ${ }^{7}$ predicts the [101] pseudo-cubic peak of $\mathrm{SrTiO}_{3}$ at $45^{\circ}$. The $\mathrm{O}$ 1s position for $\mathrm{SrTiO}_{3}$ perfectly matches this expected peak position. The [101] pseudo-cubic peak of strained $\mathrm{SrRuO}_{3}$ on $\mathrm{SrTiO}_{3}$ is expected at $44.6^{\circ}$. However, the observed value was $45.3^{\circ}$. For $\mathrm{SrRuO}_{3}$ on $\mathrm{DyScO}_{3}$ this [101] peak is expected at $45.6^{\circ}$, but was observed at $46^{\circ}$. Thus, the predicted peak positions based on a simple forward focusing approximation did not match the experimental results. However, the observed $\theta$ peak position for $\mathrm{SrRuO}_{3}$ on $\mathrm{DyScO}_{3}$ is larger than the observed peak position for $\mathrm{SrRuO}_{3}$ on $\mathrm{SrTiO}_{3}$, which agrees qualitatively with the forward focusing approximation. Possible deviations might be caused by structural changes not taken into account in this simple pseudo-cubic approximation.

\subsection{Capping layer induced enhancement of ferro- magnetism}

The above calculations and experimental data indicate the apparent sensitivity of $\mathrm{SrRuO}_{3}$ thin films to strain. Although no unambiguous experimental evidence for the predicted structural phase transition was found, $\mathrm{SrRuO}_{3}$ seems to be highly sensitive to the applied strain, resulting in tilting of the oxygen octahedra. Therefore, an interesting route to study the effects of strain and octahedral coupling separately is to apply a capping layer to ultra-thin $\mathrm{SrRuO}_{3}$ films to influence the octahedral rotations. This capping-layer methodology is studied below. Experimental results of the effect of the capping layer on the magnetic properties are provided and supporting DFT calculations are discussed.

\subsection{1 $\mathrm{T}_{\mathrm{C}}$ enhancement}

The ferromagnetic $T_{C}$ was studied as a function of film thickness and capping layer thickness using the polar magneto-optic Kerr effect (MOKE). The polar Kerr effect was used for studying out-of-plane magnetism. ${ }^{[144]}$ MOKE is a non-

\footnotetext{
${ }^{7}$ The expected peak position is calculated using: $\tan \theta=\frac{a}{c}$. For strained $\mathrm{SrRuO}_{3}$ films lattice constants are calculated by fixing the in-plane lattice parameters to the values of the substrate and calculating the c-axis by assuming the volume of the $\mathrm{SrRuO}_{3}$ unit-cell is conserved.
} 
Figure temporarily withheld from online publication. This figure is prepared for publication by Thomas et al. ${ }^{[136]}$. Corresponding authors G. Koster and J. Xia.

Figure 7.6: $\mathrm{T}_{C}$ as a function of temperature for $\mathrm{SrRuO}_{3}$ films in (a). $\mathrm{SrTiO}_{3}$ capped films on $\mathrm{DyScO}_{3}$ substrates had a higher $\mathrm{T}_{C}$ than uncapped films. The $\mathrm{T}_{C}$ increase as a function of film thickness is indicated by the dotted red line (a guide to the eye). In (b) a schematic of the Kerr measurement setup is shown and in (c) an AFM image of a 10 u.c. $\mathrm{SrRuO}_{3}$ film grown on a $\mathrm{DyScO}_{3}$ substrate capped with 8 u.c. of $\mathrm{SrTiO}_{3}$ is shown. (d) An example of an hysteresis measurement of an uncapped 7 u.c. $\mathrm{SrRuO}_{3}$ film on a $\mathrm{DyScO}_{3}$ substrate. (e) A comparison of Kerr signal as a function of the temperature for capped an uncapped 10 u.c. $\mathrm{SrRuO}_{3}$ films on $\mathrm{DyScO}_{3}$ substrates. The figure is adapted from Thomas et al. ${ }^{136]}$.

reciprocal effect where left- and right-circularly polarized light traveling through a magnetic medium propagate at different speeds. ${ }^{[149]}$ Left- and right-circularly polarized light acquire a phase different equal to twice the Kerr signal. The Kerr signal is proportional to the magnetic moment of the of sample. In the polar Kerr measurement, light is reflected perpendicular to the surface. In ultra-thin films, the penetration depth of light is much larger than the film thickness. Therefore, the Kerr signal is directly proportional to the density of magnetic moments in the film. ${ }^{[136,150]}$

\section{MOKE results}

Experimentally observed $\mathrm{T}_{C}$ values for $\mathrm{SrRuO}_{3}$ films on $\mathrm{SrTiO}_{3}$ and $\mathrm{DyScO}_{3}$ are shown in Fig. 7.6(a). The red markers indicate previous obtained results of uncapped $\mathrm{SrRuO}_{3}$ films on $\mathrm{SrTiO}_{3}$ substrates. A dotted red line is added to the data as a guide to eye, indicating the observed dependence of $\mathrm{T}_{C}$ for uncapped films on $\mathrm{SrTiO}_{3}$. On $\mathrm{SrTiO}_{3}$, a TC values of $109 \mathrm{~K}$ for 4 u.c. of $\mathrm{SrRuO}_{3}$ was found and a $\mathrm{T}_{C}$ of $146 \mathrm{~K}$ for a 20 u.c. film. Uncapped $\mathrm{SrRuO}_{3}$ films on $\mathrm{DyScO}_{3}$ showed a lower $\mathrm{T}_{C}$ as function of temperature compared to $\mathrm{SrTiO}_{3}$, as indicated by the black markers. Although a thicker, 35 u.c. film of $\mathrm{SrRuO}_{3}$ on $\mathrm{DyScO}_{3}$ was found to have a $\mathrm{T}_{C}$ of $158 \mathrm{~K}$, i.e., very close to the bulk transition temperature. For all films below 4 u.c. no ferromagnetic order was found, as was previously shown. ${ }^{[142]}$

Several films were prepared with an additional $\mathrm{SrTiO}_{3}$ capping layer. For example a 10 u.c. film grown on a $\mathrm{DyScO}_{3}$ substrate capped with 8 u.c. of $\mathrm{SrTiO}_{3}$ had a $\mathrm{T}_{C}$ of $154 \mathrm{~K}$; more than $20 \mathrm{~K}$ higher than after removing the capping layer 
by wet chemical etching (see below). Similarly, a 7 u.c. $\mathrm{SrRuO}_{3}$ film on $\mathrm{DyScO}_{3}$ capped with 8 u.c. of $\mathrm{SrTiO}_{3}$ showed in increase of more than $20 \mathrm{~K}$ as well. No noticeable change in $\mathrm{T}_{C}$ was found when applying a capping layer on $\mathrm{SrTiO}_{3}$ substrates. For example, two different samples of 5 u.c. $\mathrm{SrRuO}_{3}$ were prepared, one with a 2 u.c. capping layer and one without capping. Both samples had similar $\mathrm{T}_{C}$ as indicated in Fig. 7.6(a) by the blue dot.

Different values of the capping layer thickness, shown in Fig. 7.6(a), were obtained either by fabricating different films or by selective chemical etching of the $\mathrm{SrTiO}_{3}$ capping-layer. This selective etching was done using a $\mathrm{HF}-\mathrm{HNO}_{3}$ and water mixture, which etches $\mathrm{SrTiO}_{3}$, but does not affect $\mathrm{SrRuO}_{3}$ and $\mathrm{DyScO}_{3}$. ${ }^{\text {[138] }}$ The etching was done while partially covering the sample surface, resulting in a partial coverage of the capping layer on one substrate. A schematic of the resulting surface configuration and Kerr measurement is shown in Fig. 7.6(b). The surface of a 10 u.c. $\mathrm{SrRuO}_{3}$ film capped with 8 u.c. $\mathrm{SrTiO}_{3}$ on $\mathrm{DyScO}_{3}$ was atomically smooth, as shown in Fig. 7.6(c). A comparison of the $\mathrm{T}_{C}$ as a function of temperature before and after etching is shown in Fig. 7.6(e), which clearly showed an increased $\mathrm{T}_{C}$ prior to removal of the capping layer. For reference, an example hysteresis curve of $\mathrm{SrRuO}_{3}$ is shown in Fig. 7.6(d).

By decreasing the capping layer thickness on a different sample of 10 u.c. of $\mathrm{SrRuO}_{3}$ on $\mathrm{DyScO}_{3}$ to only 2 layers of $\mathrm{SrTiO}_{3}$, the $\mathrm{T}_{C}$ enhancement was reduced by a factor of two. This indicated that the $\mathrm{T}_{C}$ enhancement was dependent on the capping layer thickness. However, the enhancement saturated close to 8 u.c. of capping layer. Adding 20 u.c. of $\mathrm{SrTiO}_{3}$ capping to a 10 u.c. $\mathrm{SrRuO}_{3}$ film on $\mathrm{DyScO}_{3}$ did not further enhance $\mathrm{T}_{C}$. By means of chemical etching, an area of 1 u.c. $\mathrm{SrTiO}_{3}$ coverage was acquired, which only had a slightly lowered $\mathrm{T}_{C}$ compared to areas covered with 2 u.c, indicating the initial capping layer had the largest influence on $\mathrm{T}_{C}$, see also section 7.3.2.

\section{DFT calculations}

In order to understand how strain and capping influences the $\mathrm{T}_{C}, \mathrm{DFT}$ calculations were performed. By optimizing the lattice constant of $\mathrm{SrRuO}_{3}$, a halfmetallic state was found with a total magnetic moment of $1.97 \mu \mathrm{B}$, which agreed with previous calculations and experimental measurements. ${ }^{[151,152]}$ The effects of strain were simulated in three scenarios were an in-plane strain $(\varepsilon)$ was applied from $-2 \%$ to $+2 \%$. The exchange energy $\left(\Delta E=E_{A F M}-E_{F M}\right)$ shown in Fig. 7.7, is a measure for $T_{C}$. A positive exchange coupling is indicative of a ferromagnet and a negative exchange energy of an anti-ferromagnet. The black curve (first scenario) represents the results obtained by only applying in-plane strain, the red curve (second scenario) was obtained by also fixing the c-axis in order to conserve the volume in the unit-cell and the blue curve (third scenario) is obtained in a similar way, but now applying an $\mathrm{SrTiO}_{3}$ capping layer. The in-plane strain applied by $\mathrm{SrTiO}_{3}(-0.46 \%)$ and $\mathrm{DyScO}_{3}(+0.66 \%)$ are indicated by the shaded regions.

Maximum $\triangle \mathrm{E}$ was observed for bulk (black curve) and free standing thin films (red curve) of $\mathrm{SrRuO}_{3}$ under compressive strain. This helps to explain the experi- 


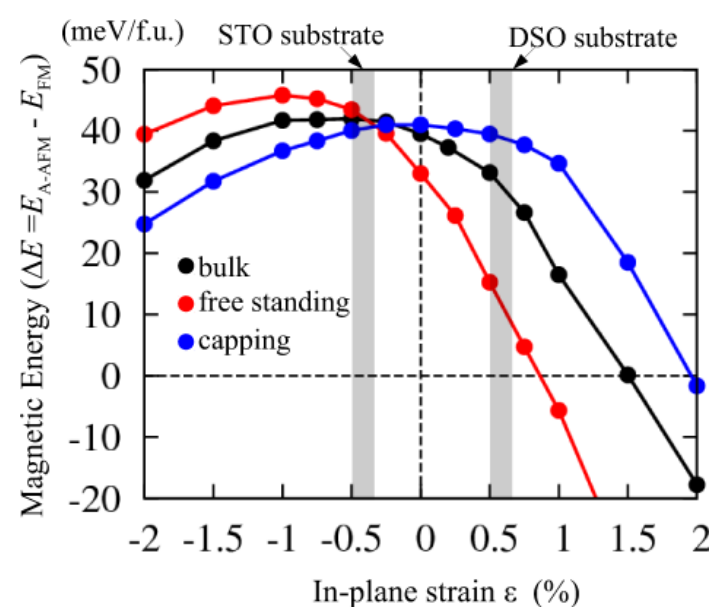

Figure 7.7: Magnetic energy $\Delta E=E_{A-A F M}-E_{F M}$ of bulk $\mathrm{SrRuO}_{3}$ (black), free standing $\mathrm{SrRuO}_{3}$ thin films (red), and $\mathrm{SrRuO}_{3}$ thin films capped with $\mathrm{SrTiO}_{3}$ (blue), with respect to in-plane strain $\varepsilon$. Shaded regions indicate in-plane strain induced by $\mathrm{SrTiO}_{3}$ or $\mathrm{DyScO}_{3}$ substrate. The DFT calculations were performed by Zhicheng Zhong. [136]

mental results, where $\mathrm{SrRuO}_{3}$ thin films $\mathrm{DyScO}_{3}$ showed a reduced $\mathrm{T}_{C}$ compared to films on $\mathrm{SrTiO}_{3}$. The addition of an $\mathrm{SrTiO}_{3}$ capping layer to a $\mathrm{SrRuO}_{3}$ film on $\mathrm{DyScO}_{3}$ might effectively reduce the applied tensile strain $(\varepsilon)$ resulting in a higher $\mathrm{T}_{C}$. This also helps to explain the experimental data, whereas the thickness of the $\mathrm{SrTiO}_{3}$ capping layer was increased, the $\mathrm{T}_{C}$ enhancement was larger. However, the effect of the capping layer also introduced an additional boundary condition on the oxygen octahedra at the interface between $\mathrm{SrRuO}_{3}$ and $\mathrm{SrTiO}_{3}$.

The interfaces between the capping-layer, $\mathrm{SrRuO}_{3}$ film and substrate had an effect on the rotation and distortions of the $\mathrm{RuO}_{6}$ octahedra. ${ }^{[15]}$ Due to strong hybridization between the ruthenium d-orbitals and the oxygen p-orbitals, the rotations of the oxygen octahedra in $\mathrm{SrRuO}_{3}$ can have a large effect on the electronic and magnetic properties. ${ }^{[153]}$ Calculation results including this interface coupling effect are shown blue in Fig. 7.7 (blue curve). For compressive strain, capping reduced the $\mathrm{T}_{C}$ slightly. However at just $-0.46 \%$ strain induced by $\mathrm{SrTiO}_{3}$, this effect is negligible. This was also observed in the experimental data where capping $\mathrm{SrRuO}_{3}$ on $\mathrm{SrTiO}_{3}$ with $\mathrm{SrTiO}_{3}$ did not influence $\mathrm{T}_{C}$. For tensile strain, like on $\mathrm{DyScO}_{3}$, the addition of a capping layer had a larger effect on $\Delta \mathrm{E}$, which indicated an increase in $\mathrm{T}_{C}$ when adding a capping layer.

The observed difference in behavior of the capped and free-standing situations can be explained by the type of the distortions of the oxygen octahedra. In Table 7.2 the tilt and rotations angles of bulk, free-standing thin films and capped $\mathrm{SrRuO}_{3}$ films are shown. The main effect of strain on bulk and thin films of $\mathrm{SrRuO}_{3}$ was to change the $\mathrm{Ru}-\mathrm{O}$ bonds lengths (c-axis) due to a change in height of the unit-cell, while the tilt and rotation angles of the oxygen octahedra remained nearly constant when the strain was varied. This effect is shown in 
Table 7.2: Octahedral tilt and rotation angles (degrees) for the three different scenarios under in-plane strain: (1) bulk in-plane strained; (2) thin film in-plane strained and (3) an in-plane strained thin film with a capping layer. A sketch of the tilt and rotation angles is shown in Fig. 7.1. For the thin film scenarios the angles are mentioned for the first and second layers. The second layer values are close to bulk; relaxation occurs quickly. The tilt and rotation angles for the first two scenarios are almost constant as a function of strain. However, for the capped thin films these angles vary more strongly as a function of strain. The DFT calculations were performed by Zhicheng Zhong, see section 2.4.2.

\begin{tabular}{|c|c|c|c|c|c|c|}
\hline & \multicolumn{2}{|c|}{$\overline{\bar{\varepsilon} \varepsilon=0 \%}$} & \multicolumn{2}{|c|}{ 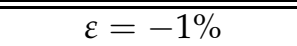 } & \multicolumn{2}{|c|}{$\overline{c \varepsilon=1 \%}$} \\
\hline & Tilt $\theta$ & Rotation $\phi$ & Tilt $\theta$ & Rotation $\phi$ & Tilt $\theta$ & Rotation $\phi$ \\
\hline Bulk & $9.8^{\circ}$ & $8.0^{\circ}$ & $9.5^{\circ}$ & $8.0^{\circ}$ & $9.05^{\circ}$ & $7.95^{\circ}$ \\
\hline Thin film $1^{e}$ & $7.6^{\circ}$ & $9.8^{\circ}$ & $7.7^{\circ}$ & $9.6^{\circ}$ & $7.6^{\circ}$ & $9.7^{\circ}$ \\
\hline Thin film $2^{e}$ & & $8.5^{\circ}$ & & $8.6^{\circ}$ & & $8.5^{\circ}$ \\
\hline Capping $1^{e}$ & $5.6^{\circ}$ & $7.4^{\circ}$ & $5.5^{\circ}$ & $6.5^{\circ}$ & $6.5^{\circ}$ & $6.3^{\circ}$ \\
\hline Capping $2^{e}$ & $9.4^{\circ}$ & $8.5^{\circ}$ & $8.95^{\circ}$ & $8.5^{\circ}$ & $9.2^{\circ}$ & $8.5^{\circ}$ \\
\hline
\end{tabular}

Table 7.2, where almost no change in tilt and rotation angles were observed for bulk $\mathrm{SrRuO}_{3}$ as a function of strain. However, introducing a $\mathrm{SrTiO}_{3}$ capping layer had a large impact on the role of strain on the tilt and rotation angles. Compared to bulk and uncapped thin films the rotations were reduced in the case of capping for all values of applied strain. Moreover, for different strain values, the rotation and tilt angles vary more strongly compared to bulk and uncapped thin films. Rotation angles of the second $\mathrm{SrRuO}_{3}$ layer from the interface are close to bulk values, indicating relaxation occurs quickly. ${ }^{8}$

\subsubsection{Selective etching of the $\mathrm{SrTiO}_{3}$ capping layer}

As discussed briefly in section 7.3.1 the $\mathrm{SrTiO}_{3}$ capping layer was selectively etched using a acidic solution. ${ }^{[138]}$ By applying a partial coverage with wax, the etching was performed on selected areas of the substrate. Results of such an etch are shown in Fig. 7.8(a) of a 10 u.c. $\mathrm{SrRuO}_{3}$ film on $\mathrm{DyScO}_{3}$ capped 2 u.c. of $\mathrm{SrTiO}_{3}$. In Fig. 7.8(a) three distinct regions are visible in the false color spatial map of the Kerr signal with the same reflected power (not shown). For the defined regions, $\mathrm{T}_{C}$ values of 131,141 and $144 \mathrm{~K}$ were measured. The latter value of $144 \mathrm{~K}$ was equal to the value observed prior to etching, i.e., 2 u.c. of capping layer (region 3). The other regions were attributed to 1 u.c. layer of $\mathrm{SrTiO}_{3}$ capping (region 2) and no capping (region 1). The temperature dependence of the Kerr signal is plotted for the three regions in Fig. 7.8(b). The $\mathrm{T}_{C}$ difference between no capping layer (region 1) and one layer (region 2) is about $10 \mathrm{~K}$, while the addition of a second capping layer (region 3) only increased $\mathrm{T}_{C}$ with $4 \mathrm{~K}$.

\footnotetext{
${ }^{8}$ In the calculations the tilt and rotations angles quickly return to their bulk values, as indicated by the difference between the surface layer rotations and the second layer rotations. However, in a recent experiment involving an $\mathrm{SrRuO}_{3}-\mathrm{GdScO}_{3}$ interface, it was demonstrated that the tilt and rotation angles in the $\mathrm{SrRuO}_{3}$ relax to their bulk values only after 4 to 5 u.c. of film. ${ }^{[14]}$
} 


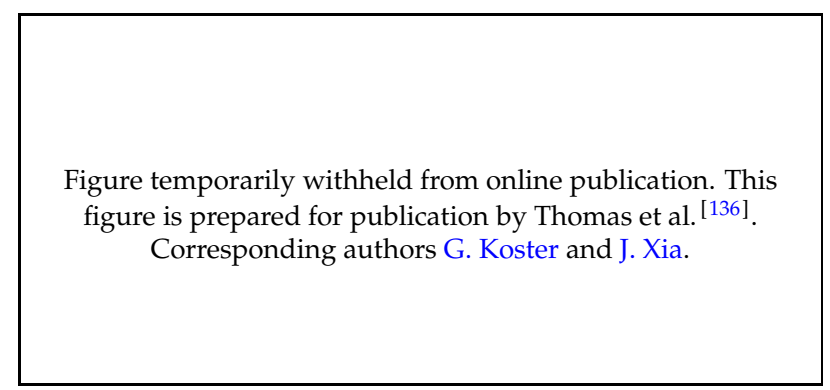

Figure 7.8: Spatial Kerr measurements (a) on a 10 u.c. $\mathrm{SrRuO}_{3}$ film on $\mathrm{DyScO}_{3}$ capped 2 u.c. of $\mathrm{SrTiO}_{3}$, where the capping layer was partially removed. Near the transition between etched and unetched areas, three distinct regions could be defined based on the Kerr signal, R1, R2 and R3. These regions were attributed to different capping layer thicknesses, zero, one and two $\mathrm{SrTiO}_{3}$ layers respectively. In (b) the temperature dependence of the Kerr signal is shown, indicating different values of $\mathrm{T}_{C}$ for the different regions. The obtained $\mathrm{T}_{\mathrm{C}}$ values are plotted in Fig. 7.6(a) as black, green and blue squares. This figure is adapted from Thomas et al. ${ }^{[136]}$.

Most of the data obtained for uncapped $\mathrm{SrRuO}_{3}$ films on $\mathrm{DyScO}_{3}$ was obtained by etching away the $\mathrm{SrTiO}_{3}$ capping layer. Therefore, the effect of etching on the $\mathrm{SrRuO}_{3}$ film was studied on a 7 u.c. $\mathrm{SrRuO}_{3}$ film without a capping layer, as shown in Fig. 7.9(a-d). The unetched region shown in (a) shows a uniform distribution of the Kerr signal, except for some small defects. Figure 7.9(c) and (d) show that the temperature dependence of the Kerr signal indicated a $\mathrm{T}_{\mathrm{C}}$ of $123 \mathrm{~K}$ for both the unetched and the etched regions. The reflected power, shown in the insets in (c) and (d), as well as the observed Kerr signal was slightly reduced on the etched region and the variation on the Kerr signal strength at low temperatures was increased with respect to the unetched region. However, a similar level of uniformity was observed and the measured $\mathrm{T}_{C}$ value was unchanged, $123 \mathrm{~K}$. Indicating the $\mathrm{T}_{C}$ values in $\mathrm{SrRuO}_{3}$ films were not influenced by the etching procedure.

\subsection{Discussion}

Thin films of $\mathrm{SrRuO}_{3}$ appear to be highly sensitive to the applied strain and interface coupling as shown by both theoretical calculations, as well as experimental data. Especially when influencing the interface coupling of octahedra by the addition of a capping layer this effect becomes clear in experimental observations of $\mathrm{T}_{C}$.

\subsubsection{Ground state of ultra-thin $\mathrm{SrRuO}_{3}$ films}

The critical thickness behavior of $\mathrm{SrRuO}_{3}$, i.e., the absence of ferromagnetism and conductivity when the $\mathrm{SrRuO}_{3}$ film thickness on $\mathrm{SrTiO}_{3}$ is reduced to below 
Figure temporarily withheld from online publication. This figure is prepared for publication by Thomas et al. ${ }^{[136]}$.

Corresponding authors G. Koster and J. Xia.

Figure 7.9: Study of $T_{C}$ in etched and unetched regions of an uncapped 7 u.c. $\mathrm{SrRuO}_{3}$ film on $\mathrm{DyScO}_{3}$. A scan of Kerr signal in an unetched region of sample at $80 \mathrm{~K}$ in (a) and a scan of the Kerr signal at $80 \mathrm{~K}$ of a partially etched region in (b), showing both etched and unetched areas. The black dots in (a) and (b) show the locations used for measuring Kerr versus temperature data in (c) and (d). Both are showing roughly the same $\mathrm{T}_{C}$ on all locations. The insets in (c) and (d) show the reflected power. Figure is adapted from Thomas et al. ${ }^{[136]}$.

4 u.c. was initially observed to also show exchange bias behavior. ${ }^{[142]}$ Exchange bias coupling occurs when a ferromagnetic and an anti-ferromagnetic materials are in contact, indicating a possible AFM phase or layer in the $\mathrm{SrRuO}_{3}$ film. DFT results, as shown in Fig. 7.2, indicate an AFM ground state of tetragonal $\mathrm{SrRuO}_{3}$. This tetragonal ground state could possibly be stabilized below 4 u.c. of $\mathrm{SrRuO}_{3}$ film thickness.

This possible tetragonal ground state was studied experimentally using high resolution (in-plane) XRD. XRD scans of half-order peaks of $\mathrm{SrRuO}_{3}$ on $\mathrm{SrTiO}_{3}$ films above and below the critical thickness show a different degree of symmetry, i.e., the 2 u.c. sample showed a more symmetric structure in the out-ofplane direction. However, a thick $\mathrm{SrRuO}_{3}$ film also showed this more symmetric structure, making the interpretation of the XRD results difficult with the limited amount of measured reflections. A possible explanation might be that if the film thickness is reduced from bulk-like to $\sim 8$ u.c., strain induces a change in structure, lowering the symmetry. When the film thickness reduced even more, i.e., below the critical thickness, possible octahedral deformations might be induced which could have a more symmetrical tilt and rotation pattern. To determine the exact nature of the observed change in symmetry more reflections should be acquired on both samples.

Magnetic dichroism experiments (XMCD) on films above and below the critical thickness on $\mathrm{DyScO}_{3}$ indicate a ferromagnetic state above the critical thick- 
ness. However, a linear dependence of the magnetic signal versus the applied field was found, indicative of paramagnetic behavior. Linear dichroism experiments did not reveal any anti-ferromagnetic order in films above and below the critical thickness. The film below the critical thickness was found to be conducting, which is different from the case studied by Xia et al. ${ }^{[142]}$, possibly explaining why no anti-ferromagnetic order was observed as a conducting $\mathrm{SrRuO}_{3}$ film is expected to be ferromagnetic. Interestingly, the sample did not show ferromagnetism, which is directly coupled to conductivity in $\mathrm{SrRuO}_{3}$. This discrepancy cannot be explained with the current models.

\subsubsection{Capping induced $\mathrm{T}_{\mathrm{C}}$ enhancement}

A $\mathrm{T}_{C}$ enhancement of up to $20 \mathrm{~K}$ is observed when applying an $\mathrm{SrTiO}_{3}$ capping layer to a 10 u.c. $\mathrm{SrRuO}_{3}$ film on $\mathrm{DyScO}_{3}$; a $\mathrm{T}_{C}$ value of $154 \mathrm{~K}$ was observed close to bulk value. Several mechanisms could induce this effect: (1) effective strain induced by the STO over-layer; (2) octahedral-coupling across the interfaces, which alter the structure and properties of the $\mathrm{SrRuO}_{3}$ film and (3) the $\mathrm{SrTiO}_{3}$ capping layer might serve as a protective cover for the $\mathrm{SrRuO}_{3}$ film. No $\mathrm{T}_{C}$ enhancement was found after applying a 2 u.c. cap to a 5 u.c. $\mathrm{SrRuO}_{3}$ film on $\mathrm{SrTiO}_{3}$. The absence of the enhancement on $\mathrm{SrTiO}_{3}$ substrates indicates the $\mathrm{SrTiO}_{3}$ capping layer does not merely act as a protective cover, ruling out the third option. A simple model which assumes that a capping layer induces effective compressive strain, which will reduce the effective tensile strain of $\mathrm{SrRuO}_{3}$ on $\mathrm{DyScO}_{3}$ does match qualitatively with the DFT calculation results. A higher $\Delta \mathrm{E}$ is obtained when the tensile strain of $\mathrm{DyScO}_{3}$ is lowered. Moreover, such a simple model also qualitatively describes the capping layer thickness dependence, as a thicker capping layer could intuitively induce a larger change in the effective applied strain. ${ }^{9}$ However, this does not match qualitatively as the observed $\mathrm{T}_{C}$ of capped films on $\mathrm{DyScO}_{3}$ is higher than uncapped $\mathrm{SrRuO}_{3}$ films on $\mathrm{SrTiO}_{3}$. Additionally, DFT calculations indicate little to no dependence of strain on the octahedral rotations and tilts.

A model which takes into account octahedral coupling across the interfaces substrate-film and film-capping does show strong changes in octahedral tilts and rotations in $\mathrm{SrRuO}_{3}$ as a function of strain. Effectively making $\mathrm{SrRuO}_{3}$ highly sensitive to the capping layer for tensile strained films. This matches with the observed experimental data and with state-of-the-art literature studies, like done by Aso et al. ${ }^{[14]}$. Therefore, making the second mechanism the most likely scenario.

A highly reactive $\mathrm{HNO}_{3}$-HF etch was performed on several samples to locally remove the $\mathrm{SrTiO}_{3}$ capping layer as done successfully by Gan et al. ${ }^{[138]}$ on $\mathrm{SrTiO}_{3}$ substrates. In most cases the data-points of capped, uncapped and various capping layer thickness where obtained by this chemical etching method. Therefore, the influence of etching on $\mathrm{SrRuO}_{3}$ films was studied. The observed

\footnotetext{
${ }^{9}$ In these ultra-thin $\mathrm{SrRuO}_{3}$ films both the $\mathrm{SrRuO}_{3}$ and the $\mathrm{SrTiO}_{3}$ capping layer are fully strained to the substrate. The strain or deformation is therefore fixed. The additional stress possibly caused by the $\mathrm{SrTiO}_{3}$ film is referred to as effective strain in the text.
} 
change in Kerr signal intensity and reflective power is attributed to a change in sample roughness. However, the $\mathrm{T}_{C}$ did not change after etching of an uncapped film. The $T_{C}$ values obtained after etching are in qualitative agreement with the guide to the eye drawn in Fig. 7.6(a), indicating the an intrinsic uncapped film is similar to a film where the $\mathrm{SrTiO}_{3}$ capping layer is removed. The latter observation is interesting since it shows to structure and properties of the $\mathrm{SrRuO}_{3}$ film can be altered when etching is done at room temperature.

\subsection{Conclusions}

The DFT prediction of a possible tetragonal and anti-ferromagnetic (AFM) phase in $\mathrm{SrRuO}_{3}$ can explain the observed critical thickness behavior in $\mathrm{SrRuO}_{3}$ ultrathin films. The structure of ultra-thin films is shown to be more symmetric below the critical thickness when comparing films close to this transition. However, no definite statements about the octahedral tilt patterns (structure) of $\mathrm{SrRuO}_{3}$ can be made based on the current XRD and XPD experiments. Moreover, no evidence for an AFM phase was found using $X$-ray dichroism experiments.

Enhancement of $\mathrm{T}_{C}$ in $\mathrm{SrRuO}_{3}$ thin films by addition of an $\mathrm{SrTiO}_{3}$ capping layer of up to $20 \mathrm{~K}$ has been shown. This effect was observed only if the capping layer and substrate were of different materials, in this case, $\mathrm{SrTiO}_{3}$ capping of $\mathrm{SrRuO}_{3}$ grown on $\mathrm{DyScO}_{3}$ substrates. DFT calculations indicate this enhancement is due to a change in octahedral tilt angles in the $\mathrm{SrRuO}_{3}$ thin films induced by the $\mathrm{SrTiO}_{3}$ capping layer. The $\mathrm{SrTiO}_{3}$ capping layer has larger effect on $\Delta E$ when $\mathrm{SrRuO}_{3}$ is under tensile strain, as is the case on a $\mathrm{DyScO}_{3}$ substrate. This separate control of octahedral distortions and substrate induced strain resulted in a $\mathrm{T}_{C}$ near bulk value in a $4 \mathrm{~nm} \mathrm{SrRuO}$ thin film. Separate control of strain and octahedral coupling opens new pathways to enhance or find new properties in complex oxide thin films. 


\section{Bibliography}

[1] Richard P. Feynman. There's plenty of room at the bottom. Engineering and Science 23, 22 (1960).

[2] Editorial. 'Plenty of room' revisited. Nature nanotechnology 4, 781 (2009).

[3] M. N. Baibich, J. M. Broto, A. Fert, F. Nguyen Van Dau, F. Petroff, P. Etienne, G. Creuzet, A. Friederich, and J. Chazelas. Giant magnetoresistance of $(001) \mathrm{Fe} /(001) \mathrm{Cr}$ magnetic superlattices. Physical Review Letters 61, 2472 (1988).

[4] G. Binasch, P. Grünberg, F. Saurenbach, and W. Zinn. Enhanced magnetoresistance in layered magnetic structures with antiferromagnetic interlayer exchange. Physical Review B 39, 4828 (1989).

[5] D. Dijkkamp, T. Venkatesan, X. D. Wu, S. A. Shaheen, N. Jisrawi, Y. H. Min-Lee, W. L. McLean, and M. Croft. Preparation of $Y-B a-C u$ oxide superconductor thin films using pulsed laser evaporation from high Tc bulk material. Applied Physics Letters 51, 619 (1987).

[6] G. J. H. M. Rijnders, G. Koster, D. H. A. Blank, and H. Rogalla. In situ monitoring during pulsed laser deposition of complex oxides using reflection high energy electron diffraction under high oxygen pressure. Applied Physics Letters 70, 1888 (1997).

[7] M. Azuma, Z. Hiroi, M. Takano, Y. Bando, and Y. Takeda. Superconductivity at $110 \mathrm{~K}$ in the infinite-layer compound $\left(\mathrm{Sr}_{1-x} \mathrm{Ca}_{x}\right)_{1-y} \mathrm{CuO}_{2}$. Nature 356, 775 (1992).

[8] M. Takano, Y. Takeda, H. Okada, M. Miyamoto, and T. Kusaka. ACuO (A: alkaline earth) crystallizing in a layered structure. Physica C: Superconductivity 159, 375 (1989).

[9] P. Muralt, R. G. Polcawich, and S. Trolier-McKinstry. Piezoelectric thin films for sensors, actuators, and energy harvesting. MRS Bulletin 34, 658 (2011).

[10] A. Ohtomo and H. Y. Hwang. A high-mobility electron gas at the $\mathrm{LaAlO}_{3} / \mathrm{SrTiO}_{3}$ heterointerface. Nature 427, 423 (2004).

[11] J. M. Rondinelli, S. J. May, and J. W. Freeland. Control of octahedral connectivity in perovskite oxide heterostructures: An emerging route to multifunctional materials discovery. MRS Bulletin 37, 261 (2012).

[12] J. H. Haeni, P. Irvin, W. Chang, R. Uecker, P. Reiche, Y. L. Li, S. Choudhury, W. Tian, M. E. Hawley, B. Craigo, A. K. Tagantsev, X. Q. Pan, S. K. Streiffer, L. Q. Chen, S. W. Kirchoefer, J. Levy, and D. G. Schlom. Room-temperature ferroelectricity in strained $\mathrm{SrTiO}_{3}$. Nature 430, 758 (2004).

[13] J. He, A. Borisevich, S. V. Kalinin, S. J. Pennycook, and S. T. Pantelides. Control of octahedral tilts and magnetic properties of perovskite oxide heterostructures by substrate symmetry. Physical Review Letters 105, 227203 (2010).

[14] R. Aso, D. Kan, Y. Shimakawa, and H. Kurata. Atomic level observation of octahedral distortions at the perovskite oxide heterointerface. Scientific reports 3, 2214 (2013).

[15] J. M. Rondinelli and N. A. Spaldin. Substrate coherency driven octahedral rotations in perovskite oxide films. Physical Review B 82, 113402 (2010).

[16] S. Bühlmann, B. Dwir, J. Baborowski, and P. Muralt. Size effect in mesoscopic epitaxial ferroelectric structures: Increase of piezoelectric response with decreasing feature size. Applied Physics Letters 80, 3195 (2002).

[17] J. L. MacManus-Driscoll. Self-assembled heteroepitaxial oxide nanocomposite thin film structures: designing interface-induced functionality in electronic materials. Advanced Functional Materials 20, 2035 (2010). 
[18] R. Uecker, B. Velickov, D. Klimm, R. Bertram, M. Bernhagen, M. Rabe, M. Albrecht, R. Fornari, and D. G. Schlom. Properties of rare-earth scandate single crystals (Re=Nd-Dy). Journal of Crystal Growth 310, 2649 (2008).

[19] V. M. Goldschmidt. Die Gesetze der Krystallochemie. Die Naturwissenschaften 14, 477 (1926).

[20] M. Schmidbauer, A. Kwasniewski, and J. Schwarzkopf. High-precision absolute lattice parameter determination of $\mathrm{SrTiO}_{3}, \mathrm{DyScO}_{3}$ and $\mathrm{NdGaO}_{3}$ single crystals. Acta Crystallographica Section B 68, 8 (2012).

[21] M. Raekers, K. Kuepper, S. Bartkowski, M. Prinz, A. V. Postnikov, K. Potzger, S. Zhou, A. Arulraj, N. Stüß er, R. Uecker, W. L. Yang, and M. Neumann. Electronic and magnetic structure of $\mathrm{RScO}_{3}(\mathrm{R}=\mathrm{Sm}, \mathrm{Gd}, \mathrm{D} y)$ from $\mathrm{X}$-ray spectroscopies and first-principles calculations. Physical Review $\mathrm{B}$ 79, 125114 (2009).

[22] D. G. Schlom, L.-Q. Chen, C.-B. Eom, K. M. Rabe, S. K. Streiffer, and J.-M. Triscone. Strain tuning of ferroelectric thin films. Annual Review of Materials Research 37, 589 (2007).

[23] M. Ohring. Epitaxy. In Materials Science of Thin Films 417-494. (2002).

[24] A. M. Glazer. The classification of tilted octahedra in perovskites. Acta Crystallographica Section B 28, 3384 (1972).

[25] G. Koster, B. L. Kropman, G. J. H. M. Rijnders, D. H. A. Blank, and H. Rogalla. Quasi-ideal strontium titanate crystal surfaces through formation of strontium hydroxide. Applied Physics Letters 73, 2920 (1998).

[26] J. E. Kleibeuker, G. Koster, W. Siemons, D. Dubbink, B. Kuiper, J. L. Blok, C. Yang, J. Ravichandran, R. Ramesh, J. E. ten Elshof, D. H. A. Blank, and G. Rijnders. Atomically defined rare-earth scandate crystal surfaces. Advanced Functional Materials 20, 3490 (2010).

[27] M. Huijben. Interface engineering for oxide electronics. PhD thesis University of Twente Enschede, The Netherlands (2006).

[28] W. Unertl, editor. Physical Structure volume 1. Elsevier 1st editio edition (1996).

[29] G. Koster. Electron diffraction techniques for studying thin film growth in situ. In G. Koster and G. Rijnders, editors, In situ characterization of thin film growth 1 . Woodhead Publishing, Limited (2011).

[30] R. Egoavil, H. Tan, J. Verbeeck, S. Bals, B. Smith, B. Kuiper, G. Rijnders, G. Koster, and G. Van Tendeloo. Atomic scale investigation of a $\mathrm{PbTiO}_{3} / \mathrm{SrRuO} / \mathrm{DyScO}$ heterostructure. Applied Physics Letters 102, 223106 (2013).

[31] P. Tinnemans. $\mathrm{DyScO}_{3}$ thin film substrates, an X-ray diffraction study. PhD thesis Radboud University Nijmegen, The Netherlands (2012).

[32] E. Vlieg. A (2+3)-type surface diffractometer: Mergence of the z-axis and (2+2)-type geometries. Journal of Applied Crystallography 31, 198 (1998).

[33] E. Vlieg. ROD: a program for surface X-ray crystallography. Journal of Applied Crystallography 33, 401 (2000).

[34] D. Briggs and J. T. Grant, editors. Surface analysis by Auger and X-ray photoelectron spectroscopy. IM Publications and SurfaceSpectra Limited (2003).

[35] J. M. Luque, M. D. Calzada, and M. Saez. A new procedure for obtaining the Voigt function dependent upon the complex error function. Journal of Quantitative Spectroscopy and Radiative Transfer 94, 151 (2005).

[36] F. J. García de Abajo, M. A. Van Hove, and C. S. Fadley. Multiple scattering of electrons in solids and molecules: A cluster-model approach. Physical Review B 63, 075404 (2001).

[37] M.-L. Xu and M. A. van Hove. Surface structure determination with forward focused electrons. Surface Science 207, 215 (1989).

[38] W. F. Egelhoff Jr. X-Ray photoelectron and auger electroo forward scattering: A new tool for surface crystallography. Critical Reviews in Solid State and Materials Sciences 16, 213 (1990). 
[39] H. C. Poon and S. Y. Tong. Focusing and diffraction effects in angle-resolved X-ray photoelectron spectroscopy. Physical Review B 30, 6211 (1984).

[40] J. F. Moulder, W. F. Stickle, P. E. Sobol, and K. D. Bomben. Handbook of X-ray photoelectron spectroscopy. Perkin-Elmer Corporation Minnesota, USA (1992).

[41] A. Herrera-Gomez, F. S. Aguirre-Tostado, P. G. Mani-Gonzalez, M. Vazquez-Lepe, A. Sanchez-Martinez, O. Ceballos-Sanchez, R. M. Wallace, G. Conti, and Y. Uritsky. Instrument-related geometrical factors affecting the intensity in XPS and ARXPS experiments. Journal of Electron Spectroscopy and Related Phenomena 184, 487 (2011).

[42] J. Xia, E. Schemm, G. Deutscher, S. A. Kivelson, D. A. Bonn, W. N. Hardy, R. Liang, W. Siemons, G. Koster, M. M. Fejer, and A. Kapitulnik. Polar Kerr-effect measurements of the high-temperature $\mathrm{YBa}_{2} \mathrm{Cu}_{3} \mathrm{O}_{6+x}$ superconductor: Evidence for broken symmetry near the pseudogap temperature. Physical Review Letters 100, 127002 (2008).

[43] J. Xia, V. Shelukhin, M. Karpovski, A. Kapitulnik, and A. Palevski. Inverse proximity effect in superconductor-ferromagnet bilayer structures. Physical Review Letters 102, 087004 (2009).

[44] H. Karapetyan, M. Hücker, G. D. Gu, J. M. Tranquada, M. M. Fejer, J. Xia, and A. Kapitulnik. Magneto-optical measurements of a cascade of transitions in superconducting $\mathrm{La}_{1.875} \mathrm{Ba}_{0.125} \mathrm{CuO}_{4}$ single crystals. Physical Review Letters 109, 147001 (2012).

[45] M. Matsumoto and T. Nishimura. Mersenne twister: a 623-dimensionally equidistributed uniform pseudo-random number generator. ACM Transactions on Modeling and Computer Simulation 8, 3 (1998).

[46] P. A. Maksym. Fast Monte Carlo simulation of MBE growth. Semiconductor Science and Technology 3, 594 (1988).

[47] J. E. Kleibeuker, B. Kuiper, S. Harkema, D. H. A. Blank, G. Koster, G. Rijnders, P. Tinnemans, E. Vlieg, P. B. Rossen, W. Siemons, G. Portale, J. Ravichandran, J. M. Szepieniec, and R. Ramesh. Structure of singly terminated polar $\mathrm{DyScO}_{3}$ (110) surfaces. Physical Review B 85, 165413 (2012).

[48] V. Vonk, S. Konings, G. J. van Hummel, S. Harkema, and H. Graafsma. The atomic surface structure of $\mathrm{SrTiO}_{3}$ (001) in air studied with synchrotron X-rays. Surface Science 595, 183 (2005).

[49] A. Fragneto, G. M. De Luca, R. Di Capua, U. Scotti di Uccio, M. Salluzzo, X. Torrelles, T.-L. Lee, and J. Zegenhagen. Ti- and Sr-rich surfaces of $\mathrm{SrTiO}_{3}$ studied by grazing incidence $x$-ray diffraction. Applied Physics Letters 91, 101910 (2007).

[50] M. R. Castell. Scanning tunneling microscopy of reconstructions on the $\mathrm{SrTiO}_{3}$ (001) surface. Surface Science 505, 1 (2002).

[51] T. Ohnishi, K. Shibuya, M. Lippmaa, D. Kobayashi, H. Kumigashira, M. Oshima, and $\mathrm{H}$. Koinuma. Preparation of thermally stable $\mathrm{TiO}_{2}$-terminated $\mathrm{SrTiO}_{3}(100)$ substrate surfaces. Applied Physics Letters 85, 272 (2004).

[52] J. G. Connell, B. J. Isaac, G. B. Ekanayake, D. R. Strachan, and S. S. A. Seo. Preparation of atomically flat $\mathrm{SrTiO}_{3}$ surfaces using a deionized-water leaching and thermal annealing procedure. Applied Physics Letters 101, 251607 (2012).

[53] G. J. Rijnders, G. Koster, D. H. Blank, and H. Rogalla. In-situ growth monitoring during PLD of oxides using RHEED at high oxygen pressure. Materials Science and Engineering: B 56, 223 (1998).

[54] R. Bachelet, F. Sánchez, F. J. Palomares, C. Ocal, and J. Fontcuberta. Atomically flat SrO-terminated $\mathrm{SrTiO}_{3}$ (001) substrate. Applied Physics Letters 95, 141915 (2009).

[55] X. Ke, C. Adamo, D. G. Schlom, M. Bernhagen, R. Uecker, and P. Schiffer. Low temperature magnetism in the perovskite substrate $\mathrm{DyScO}_{3}$. Applied Physics Letters 94, 152503 (2009).

[56] M. Foerster, R. Bachelet, V. Laukhin, J. Fontcuberta, G. Herranz, and F. Sánchez. Laterally confined two-dimensional electron gases in self-patterned $\mathrm{LaAlO}_{3} / \mathrm{SrTiO}_{3}$ interfaces. Applied Physics Letters 100, 231607 (2012).

[57] B. Kuiper, J. L. Blok, H. J. W. Zandvliet, D. H. A. Blank, G. Rijnders, and G. Koster. Selforganization of $\mathrm{SrRuO}_{3}$ nanowires on ordered oxide surface terminations. MRS Communications 
1, 17 (2011).

[58] R. Bachelet, F. Sánchez, J. Santiso, C. Munuera, C. Ocal, and J. Fontcuberta. Self-assembly of $\mathrm{SrTiO}_{3}$ (001) chemical-terminations: A route for oxide-nanostructure fabrication by selective growth. Chemistry of Materials 21, 2494 (2009).

[59] J. E. Boschker and T. Tybell. Qualitative determination of surface roughness by in situ reflection high energy electron diffraction. Applied Physics Letters 100, 151604 (2012).

[60] G. Koster, G. Rijnders, D. H. A. Blank, and H. Rogalla. Surface morphology determined by (001) single-crystal $\mathrm{SrTiO}_{3}$ termination. Physica C: Superconductivity 339, 215 (2000).

[61] P. Jakob and Y. J. Chabal. Chemical etching of vicinal Si(111): Dependence of the surface structure and the hydrogen termination on the $\mathrm{pH}$ of the etching solutions. The Journal of Chemical Physics 95, 2897 (1991).

[62] R. P. Liferovich and R. H. Mitchell. A structural study of ternary lanthanide orthoscandate perovskites. Journal of Solid State Chemistry 177, 2188 (2004).

[63] A. Biswas, P. B. Rossen, C.-H. Yang, W. Siemons, M.-H. Jung, I. K. Yang, R. Ramesh, and $\mathrm{Y}$. H. Jeong. Universal Ti-rich termination of atomically flat $\mathrm{SrTiO}_{3}(001),(110)$, and (111) surfaces. Applied Physics Letters 98, 051904 (2011).

[64] H. Boschker, J. Verbeeck, R. Egoavil, S. Bals, G. van Tendeloo, M. Huijben, E. P. Houwman, G. Koster, D. H. A. Blank, and G. Rijnders. Preventing the reconstruction of the polar discontinuity at oxide heterointerfaces. Advanced Functional Materials 22, 2235 (2012).

[65] M. N. K. Bhuiyan, M. Menghini, J.-P. Locquet, J. Won Seo, C. Dieker, W. Jäger, and C. Marchiori. Epitaxial growth of $\mathrm{Dy}_{2} \mathrm{O}_{3}$ films on $\mathrm{SrTiO}_{3}(001)$ substrates by molecular beam epitaxy. Journal of Vacuum Science and Technology B 29, 01A801 (2011).

[66] B. V. Beznosikov and K. S. Aleksandrov. Perovskite-like crystals of the Ruddlesden-Popper series. Crystallography Reports 45, 792 (2000).

[67] G. Koster, G. J. H. M. Rijnders, D. H. A. Blank, and H. Rogalla. Imposed layer-by-layer growth by pulsed laser interval deposition. Applied Physics A Materials Science and Processing 69, S17-S22 (1999).

[68] R. Dirsyte, J. Schwarzkopf, G. Wagner, R. Fornari, J. Lienemann, M. Busch, and H. Winter. Thermal-induced change in surface termination of $\mathrm{DyScO}_{3}(110)$. Surface Science 604, L55-L58 (2010).

[69] H. N. Lee, H. M. Christen, M. F. Chisholm, C. M. Rouleau, and D. H. Lowndes. Strong polarization enhancement in asymmetric three-component ferroelectric superlattices. Nature 433, 395 (2005).

[70] G. Koster, K. Verbist, G. Rijnders, H. Rogalla, G. van Tendeloo, and D. H. A. Blank. Structure and properties of $(\mathrm{Sr}, \mathrm{Ca}) \mathrm{CuO}_{2}-\mathrm{BaCuO}$ superlattices grown by pulsed laser interval deposition. Physica C: Superconductivity 353, 167 (2001).

[71] G. Rijnders, D. H. A. Blank, J. Choi, and C.-B. Eom. Enhanced surface diffusion through termination conversion during epitaxial $\mathrm{SrRuO}_{3}$ growth. Applied Physics Letters 84, 505 (2004).

[72] H. Zheng, J. Wang, S. E. Lofland, Z. Ma, L. Mohaddes-Ardabili, T. Zhao, L. Salamanca-Riba, S. R. Shinde, S. B. Ogale, F. Bai, D. Viehland, Y. Jia, D. G. Schlom, M. Wuttig, A. Roytburd, and R. Ramesh. Multiferroic $\mathrm{BaTiO}_{3}-\mathrm{CoFe}_{2} \mathrm{O}_{4}$ nanostructures. Science 303, 661 (2004).

[73] J. He, R. Dittmann, S. Karthäuser, and E. Vasco. Geometric shadowing from rippled SrRu $\mathrm{O}_{3} / \mathrm{SrTiO}_{3}$ surface templates induces self-organization of epitaxial $\mathrm{SrZrO}_{3}$ nanowires. Physical Review B 74, 205410 (2006).

[74] F. Sánchez, G. Herranz, I. C. Infante, C. Ferrater, M. V. García-Cuenca, M. Varela, and J. Fontcuberta. Growth modes and self-organization in the epitaxy of ferromagnetic $\mathrm{SrRuO}_{3}$ on $\mathrm{SrTiO}_{3}(001)$. Progress in Solid State Chemistry 34, 213 (2006).

[75] E. Vasco, R. Dittmann, S. Karthäuser, and R. Waser. Fabrication of stress-induced $\mathrm{SrRuO}_{3}$ nanostructures by pulsed laser deposition. Applied Physics A 79, 1461 (2004). 
[76] G. Herranz, F. Sánchez, N. Dix, D. Hrabovsky, I. C. Infante, J. Fontcuberta, M. V. García-Cuenca, C. Ferrater, and M. Varela. Controlled magnetic anisotropy of $\mathrm{SrRuO}_{3}$ thin films grown on nominally exact $\mathrm{SrTiO}_{3}(001)$ substrates. Applied Physics Letters 89, 152501 (2006).

[77] R. Bachelet, C. Ocal, L. Garzón, J. Fontcuberta, and F. Sánchez. Conducted growth of $\mathrm{SrRuO}_{3}$ nanodot arrays on self-ordered $\mathrm{La}_{0.18} \mathrm{Sr}_{0.82} \mathrm{Al}_{0.59} \mathrm{Ta}_{0.41} \mathrm{O}_{3}(001)$ surfaces. Applied Physics Letters 99, 051914 (2011).

[78] M. Naito and H. Sato. Reflection high-energy electron diffraction study on the $\mathrm{SrTiO}_{3}$ surface structure. Physica C: Superconductivity 229, 1 (1994).

[79] A. Vailionis, W. Siemons, and G. Koster. Room temperature epitaxial stabilization of a tetragonal phase in $\mathrm{ARuO}_{3}(\mathrm{~A}=\mathrm{Ca}$ and $\mathrm{Sr}$ ) thin films. Applied Physics Letters 93, 051909 (2008).

[80] B. F. Smith, B. Kuiper, J. E. ten Elshof, G. Rijnders, S. Bals, J. Verbeeck, G. V. Tendeloo, and G. Koster. Domain engineering in ferroelectric thin films through nano-patterned bottom electrodes. In preparation (2013).

[81] D. Kan and Y. Shimakawa. Geometric-shape-dependent structural transition behavior in (110) $\mathrm{SrRuO}_{3}$ epitaxial thin films. Journal of Applied Physics 111, 093532 (2012).

[82] J. Tersoff, A. W. Denier van der Gon, and R. M. Tromp. Critical island size for layer-by-layer growth. Physical Review Letters 72, 266 (1994).

[83] J. Choi, C. B. Eom, G. Rijnders, H. Rogalla, and D. H. A. Blank. Growth mode transition from layer by layer to step flow during the growth of heteroepitaxial $\mathrm{SrRuO}_{3}$ on (001) $\mathrm{SrTiO}_{3}$. Applied Physics Letters 79, 1447 (2001).

[84] W. Hong, H. N. Lee, M. Yoon, H. M. Christen, D. H. Lowndes, Z. Suo, and Z. Zhang. Persistent step-flow growth of strained films on vicinal substrates. Physical Review Letters 95, 095501 (2005).

[85] F. Sánchez, G. Herranz, J. Fontcuberta, M. García-Cuenca, C. Ferrater, and M. Varela. Giant step bunching from self-organized coalescence of $\mathrm{SrRuO}_{3}$ islands. Physical Review B 73, 073401 (2006).

[86] M. Yoon, H. N. Lee, W. Hong, H. M. Christen, Z. Zhang, and Z. Suo. Dynamics of step bunching in heteroepitaxial growth on vicinal substrates. Physical Review Letters 99, 055503 (2007).

[87] J. D. Weeks and G. H. Gilmer. Dynamics of Crystal Growth. Advances in Chemical Physics 40, 157 (1979).

[88] K. Malarz and A. Z. Maksymowicz. A simple solid-on-solid model of epitaxial thin films growth: surface morphology anisotropy. International Journal of Modern Physics C 10, 6 (1999).

[89] S. Schinzer, M. Sokolowski, M. Biehl, and W. Kinzel. Unconventional MBE strategies from computer simulations for optimized growth conditions. Physical Review B 60, 2893 (1999).

[90] P.-M. Lam, S. J. Liu, and C. H. Woo. Monte Carlo simulation of pulsed laser deposition. Physical Review B 66, 045408 (2002).

[91] Q. Zhang, J. Zhu, J. Tan, G. Yu, J. Wu, and D. Xiao. Monte Carlo simulation of the growth of $\mathrm{SrTiO}_{3}$ thin film with molecular source. Vacuum 81, 539 (2006).

[92] Y. Guanglong, Z. JiangGuo, Z. Jiliang, Z. Qinglei, L. Wei, T. Junzhe, and X. Dingquan. The growth simulation of $\mathrm{ABO}_{3}$ type epitaxial thin films. Integrated Ferroelectrics 78, 85 (2006).

[93] X.-j. Zheng, B. Yang, Z. Zhu, B. Wu, and Y.-1. Mao. Kinetic Monte Carlo simulation of growth of $\mathrm{BaTiO}_{3}$ thin film via pulsed laser deposition. Transactions of Nonferrous Metals Society of China 17, 1441 (2007).

[94] M. J. Aziz. Film growth mechanisms in pulsed laser deposition. Applied Physics A 93, 579 (2008).

[95] P. Petrov and W. Miller. A new kinetic Monte Carlo method for the thin film growth of perouskites. Surface Review and Letters 16, 909 (2009).

[96] Z. Zhu, X. J. Zheng, and W. Li. Submonolayer growth of $\mathrm{BaTiO}_{3}$ thin film via pulsed laser deposition: A kinetic Monte Carlo simulation. Journal of Applied Physics 106, 054105 (2009).

[97] Z. Zhu, X. J. Zheng, and W. Li. Multilayer growth of $\mathrm{BaTiO}_{3}$ thin films via pulsed laser deposition: An energy-dependent kinetic Monte Carlo simulation. Applied Surface Science 256, 5876 (2010). 
[98] S. B. Lee. Scaling in film growth by pulsed laser deposition and modulated beam deposition. Physical Review E 83, 041605 (2011).

[99] G. Rijnders. The initial growth of complex oxides. PhD thesis University of Twente Enschede, The Netherlands (2001).

[100] G. Koster, L. Klein, W. Siemons, G. Rijnders, J. S. Dodge, C.-B. Eom, D. H. A. Blank, and M. R. Beasley. Structure, physical properties, and applications of $\mathrm{SrRuO}_{3}$ thin films. Reviews of Modern Physics 84, 253 (2012).

[101] V. A. Shchukin and D. Bimberg. Spontaneous ordering of nanostructures on crystal surfaces. Reviews of Modern Physics 71, 1125 (1999).

[102] M. Ohring. Substrate surfaces and thin-film nucleation. In Materials Science of Thin Films 357-415. Academic Press San Diego second edition (2002).

[103] H. M. Christen and G. Eres. Recent advances in pulsed-laser deposition of complex oxides. Journal of physics: Condensed matter 20, 264005 (2008).

[104] R. L. Schwoebel and E. J. Shipsey. Step motion on crystal surfaces. Journal of Applied Physics 37, 3682 (1966).

[105] R. L. Schwoebel. Step motion on crystal surfaces II. Journal of Applied Physics 40, 614 (1969).

[106] G. Ehrlich and F. G. Hudda. Atomic view of surface selfdiffusion: Tungsten on tungsten. The Journal of Chemical Physics 44, 1039 (1966).

[107] M. H. Xie, S. Y. Leung, and S. Y. Tong. What causes step bunching- negative Ehrlich-Schwoebel barrier versus positive incorporation barrier. Surface Science 515, L459-L463 (2002).

[108] G. Koster. Artifcially layered oxides by pulsed laser deposition. PhD thesis University of Twente Enschede, The Netherlands (1999).

[109] D. Vvedensky, S. Clarke, K. Hugill, M. Wilby, and T. Kawamura. Growth kinetics on vicinal surfaces. Journal of Crystal Growth 99, 54 (1990).

[110] J. Wang, M. Fu, X. S. Wu, and D. Bai. Surface structure of strontium titanate. Journal of Applied Physics 105, 083526 (2009).

[111] R. I. Eglitis and D. Vanderbilt. First-principles calculations of atomic and electronic structure of $\mathrm{SrTiO}_{3}$ (001) and (011) surfaces. Physical Review B 77, 195408 (2008).

[112] H. Huang, G. H. Gilmer, and T. Díaz de la Rubia. An atomistic simulator for thin film deposition in three dimensions. Journal of Applied Physics 84, 3636 (1998).

[113] P. Zoontjens, T. P. Schulze, and S. C. Hendy. Hybrid method for modeling epitaxial growth: Kinetic Monte Carlo plus molecular dynamics. Physical Review B 76, 245418 (2007).

[114] Z. Zhong, G. Koster, and P. J. Kelly. Prediction of thickness limits of ideal polar ultrathin films. Physical Review B 85, 121411(R) (2012).

[115] B. Kuiper, D. Samal, D. H. A. Blank, J. E. ten Elshof, G. Rijnders, and G. Koster. Control of oxygen sublattice structure in ultra-thin $\mathrm{SrCuO}$ films studied by X-ray photoelectron diffraction. APL Materials 1, 042113 (2013).

[116] A. Vailionis, H. Boschker, W. Siemons, E. P. Houwman, D. H. A. Blank, G. Rijnders, and G. Koster. Misfit strain accommodation in epitaxial $\mathrm{ABO}_{3}$ perouskites: Lattice rotations and lattice modulations. Physical Review B 83, 064101 (2011).

[117] S. J. May, J.-W. Kim, J. M. Rondinelli, E. Karapetrova, N. A. Spaldin, A. Bhattacharya, and P. J. Ryan. Quantifying octahedral rotations in strained perouskite oxide films. Physical Review B 82, 014110 (2010).

[118] C. L. Jia, S. B. Mi, M. Faley, U. Poppe, J. Schubert, and K. Urban. Oxygen octahedron reconstruction in the $\mathrm{SrTiO}_{3} / \mathrm{LaAlO}_{3}$ heterointerfaces investigated using aberration-corrected ultrahigh-resolution transmission electron microscopy. Physical Review B 79, 081405(R) (2009).

[119] D. I. Woodward and I. M. Reaney. Electron diffraction of tilted perovskites. Acta Crystallographica Section B 61, 387 (2005). 
[120] A. Pancotti, N. Barrett, L. F. Zagonel, and G. M. Vanacore. Multiple scattering $x$-ray photoelectron diffraction study of the $\mathrm{SrTiO}_{3}(100)$ surface. Journal of Applied Physics 106, 034104 (2009).

[121] L. Despont, C. Koitzsch, F. Clerc, M. G. Garnier, P. Aebi, C. Lichtensteiger, J.-M. Triscone, F. J. Garcia de Abajo, E. Bousquet, and P. Ghosez. Direct evidence for ferroelectric polar distortion in ultrathin lead titanate perovskite films. Physical Review B 73, 094110 (2006).

[122] D. P. Woodruff. Surface structural information from photoelectron diffraction. Journal of Electron Spectroscopy and Related Phenomena 178-179, 186 (2010).

[123] C. Raisch, T. Chassé, C. Langheinrich, and A. Chassé. Preparation and investigation of the A-site and B-site terminated $\mathrm{SrTiO}_{3}$ (001) surface: A combined experimental and theoretical x-ray photoelectron diffraction study. Journal of Applied Physics 112, 073505 (2012).

[124] J. Kawai, K. Tamura, M. Owari, and Y. Nihei. X-ray photoelectron diffraction of $\mathrm{SrTiO}_{3}$. Journal of Electron Spectroscopy and Related Phenomena 61, 103 (1992).

[125] G. Er, S. Kikkawa, F. Kanamaru, Y. Miyamoto, S. Tanaka, M. Sera, M. Sato, Z. Hiroi, M. Takano, and Y. Bando. Structural, electrical and magnetic studies of infinite-layered $\mathrm{Sr}_{1-x} \mathrm{La}_{x} \mathrm{CuO} \mathrm{O}_{2}$ superconductor. Physica C: Superconductivity 196, 271 (1992).

[126] M. G. Smith, A. Manthiram, J. Zhou, J. B. Goodenough, and J. T. Markert. Electron-doped superconductivity at $40 \mathrm{~K}$ in the infinite-layer compound $\mathrm{Sr}_{1-y} \mathrm{Nd}_{y} \mathrm{CuO} 0_{2}$. Nature 351, 549 (1991).

[127] G. Koster, A. Brinkman, H. Hilgenkamp, A. J. H. M. Rijnders, and D. H. A. Blank. High-T(c) superconducting thin films with composition control on a sub-unit cell level; the effect of the polar nature of the cuprates. Journal of physics: Condensed matter 20, 264007 (2008).

[128] D. Samal, T. Haiyan, H. Molegraaf, B. Kuiper, W. Siemons, S. Bals, J. Verbeeck, G. Van Tendeloo, Y. Takamura, E. Arenholz, C. A. Jenkins, G. Rijnders, and G. Koster. Experimental evidence for oxygen sublattice control in polar infinite layer $\mathrm{SrCuO}_{2}$. Physical Review Letters 111, 096102 (2013).

[129] C. Aruta, C. Schlueter, T.-L. Lee, D. Di Castro, D. Innocenti, A. Tebano, J. Zegenhagen, and G. Balestrino. Interface reconstruction in superconducting $\mathrm{CaCuO} / 2 \mathrm{SrTiO}_{3}$ superlattices: $A$ hard $x$ ray photoelectron spectroscopy study. Physical Review B 87, 155145 (2013).

[130] M. Huijben, G. Koster, M. K. Kruize, S. Wenderich, J. Verbeeck, S. Bals, E. Slooten, B. Shi, H. J. A. Molegraaf, J. E. Kleibeuker, S. van Aert, J. B. Goedkoop, A. Brinkman, D. H. A. Blank, M. S. Golden, G. van Tendeloo, H. Hilgenkamp, and G. Rijnders. Defect engineering in oxide heterostructures by enhanced oxygen surface exchange. Advanced Functional Materials 23, 5240 (2013).

[131] W. Siemons, G. Koster, D. H. Blank, R. H. Hammond, T. H. Geballe, and M. R. Beasley. Tetragonal CuO: End member of the 3d transition metal monoxides. Physical Review B 79, 195122 (2009).

[132] A. Winkelmann, C. S. Fadley, and F. J. Garcia de Abajo. High-energy photoelectron diffraction: model calculations and future possibilities. New Journal of Physics 10, 113002 (2008).

[133] F. Parmigiani, L. E. Depero, T. Minerva, and J. B. Torrance. The fine structure of the Cu2p $p_{3 / 2}$ X-ray photoelectron spectra of copper oxide based compounds. Journal of Electron Spectroscopy and Related Phenomena 58, 315 (1992).

[134] M. A. van Veenendaal and G. A. Sawatzky. Nonlocal screening effects in $2 p x$-ray photoemission spectroscopy core-level line shapes of transition metal compounds. Physical Review Letters 70, 2459 (1993).

[135] J. H. Scofield. Hartree-Slater subshell photoionization cross-sections at 1254 and $1487 \mathrm{eV}$. Journal of Electron Spectroscopy and Related Phenomena 8, 129 (1976).

[136] S. Thomas, B. Kuiper, J. Hu, Z. Zhong, R. Wu, G. Rijnders, G. Koster, and J. Xia. Capping-layerinduced enhancement of ferromagnetism in ultra-thin $\mathrm{SrRuO}_{3}$ films. In preparation (2013).

[137] K. J. Choi, M. Biegalski, Y. L. Li, A. Sharan, J. Schubert, R. Uecker, P. Reiche, Y. B. Chen, X. Q. Pan, V. Gopalan, L.-Q. Chen, D. G. Schlom, and C. B. Eom. Enhancement of ferroelectricity in strained $\mathrm{BaTiO}_{3}$ thin films. Science 306, 1005 (2004).

[138] Q. Gan, R. A. Rao, C. B. Eom, J. L. Garrett, and M. Lee. Direct measurement of strain effects on magnetic and electrical properties of epitaxial $\mathrm{SrRuO}_{3}$ thin films. Applied Physics Letters 72, 978 
(1998).

[139] J. M. Rondinelli and C. J. Fennie. Octahedral rotation-induced ferroelectricity in cation ordered perovskites. Advanced Materials 24, 1961 (2012).

[140] D. C. Worledge and T. H. Geballe. Negative spin-polarization of SrRuOH. Physical Review Letters 85,5182 (2000).

[141] M. Feigenson, J. W. Reiner, and L. Klein. Efficient current-induced domain-wall displacement in $\mathrm{SrRuO}_{3}$. Physical Review Letters 98, 247204 (2007).

[142] J. Xia, W. Siemons, G. Koster, M. R. Beasley, and A. Kapitulnik. Critical thickness for itinerant ferromagnetism in ultrathin films of $\mathrm{SrRuO}_{3}$. Physical Review B 79, 140407(R) (2009).

[143] Z. Q. Liu, Y. Ming, W. M. Lü, Z. Huang, X. Wang, B. M. Zhang, C. J. Li, K. Gopinadhan, S. W. Zeng, A. Annadi, Y. P. Feng, T. Venkatesan, and Ariando. Tailoring the electronic properties of $\mathrm{SrRuO}_{3}$ films in $\mathrm{SrRuO}_{3} / \mathrm{LaAlO}_{3}$ superlattices. Applied Physics Letters 101, 223105 (2012).

[144] J. Xia, P. T. Beyersdorf, M. M. Fejer, and A. Kapitulnik. Modified Sagnac interferometer for high-sensitivity magneto-optic measurements at cryogenic temperatures. Applied Physics Letters 89, 062508 (2006).

[145] C. W. Jones, P. D. Battle, P. Lightfoot, and W. T. A. Harrison. The structure of SrRuO $\mathrm{O}_{3}$ by time-offlight neutron powder diffraction. Acta Crystallographica Section C 45, 365 (1989).

[146] K. J. Choi, S. H. Baek, H. W. Jang, L. J. Belenky, M. Lyubchenko, and C.-B. Eom. Phase-transition temperatures of strained single-crystal $\mathrm{SrRuO}_{3}$ thin films. Advanced Materials 22, 759 (2010).

[147] Y. Chang, C. Kim, S.-H. Phark, Y. Kim, J. Yu, and T. Noh. Fundamental thickness limit of itinerant ferromagnetic $\mathrm{SrRuO}_{3}$ thin films. Physical Review Letters 103, 057201 (2009).

[148] R. Vidya, P. Ravindran, A. Kjekshus, H. Fjellvåg, and B. C. Hauback. Magnetic properties of Ca-doped $\mathrm{SrRuO}_{3}$ from full-potential calculations. Journal of Solid State Chemistry 177, 146 (2004).

[149] C. C. Robinson. Electromagnetic theory of the Kerr and the Faraday effects for oblique incidence. Journal of the Optical Society of America 54, 1220 (1964).

[150] S. Bader. SMOKE. Journal of Magnetism and Magnetic Materials 100, 440 (1991).

[151] C. Etz, I. V. Maznichenko, D. Böttcher, J. Henk, A. N. Yaresko, W. Hergert, I. I. Mazin, I. Mertig, and A. Ernst. Indications of weak electronic correlations in $\mathrm{SrRuO}_{3}$ from first-principles calculations. Physical Review B 86, 064441 (2012).

[152] P. T. Barton, R. Seshadri, and M. J. Rosseinsky. Electrical and magnetic properties of the complete solid solution series between $\mathrm{SrRuO}_{3}$ and $\mathrm{LaRhO}_{3}$ : Filling $t_{2 g}$ versus tilting. Physical Review $\mathrm{B}$ 83, 064417 (2011).

[153] W. Lu, W. Dong Song, K. He, J. Chai, C.-J. Sun, G.-M. Chow, and J.-S. Chen. The role of octahedral tilting in the structural phase transition and magnetic anisotropy in $\mathrm{SrRuO}_{3}$ thin film. Journal of Applied Physics 113, 063901 (2013). 


\section{Summary}

The perovskite oxide material class comprises a vast range of interesting physical properties, like high temperature superconductivity and room temperature ferromagnetism. The common oxygen backbone in such $\mathrm{ABO}_{3}$ perovskites and the similar lattice parameters allow for creating oxide heterostructures. Materials with different intrinsic properties can be combined in order to find new or enhanced properties. Upon reducing the size of such complex oxide materials down to a few atomic layers, properties are often found to be altered with respect to their bulk counterparts. The nature of these changes in properties, either intrinsic or extrinsic, is an active field or research, driven by the industrial quest for smaller electronic devices.

Size reduction in oxide thin films can be achieved with different numbers of reduced dimensions. The most intuitive size reduction is to strongly reduce the film thickness to the point that a two-dimensional sheet of material is formed. Additional in-plane reduction of the number of finite dimensions results in onedimensional ribbons or zero-dimensional dots. In this thesis, epitaxial thin films are prepared and studied where the size or dimensionality is reduced. By studying growth kinetics during the formation of epitaxial, typically single crystalline thin films, the intrinsic effects of strain and oxygen octahedral coupling upon size reduction are studied in two scenarios. The first part of the thesis is aimed towards obtaining epitaxial nanostructures, where the lateral dimensions are reduced in systems with either zero or one finite dimension. The final part of the thesis discusses two cases of continuous two-dimensional thin films, where the film thickness is strongly reduced.

In order to obtain in-plane epitaxial nanostructures, a novel bottom-up route for thin film self-organization is explored, by exploiting the surface termination of $\mathrm{ABO}_{3}$ complex oxides. Such oxides are intrinsically layered as they consists of alternating sheets of $\mathrm{AO}$ and $\mathrm{BO}_{2}$. In the case of $\mathrm{DyScO}_{3}$ these alternating layers have opposite charge. The exact surface of a polar complex oxide substrate material $\mathrm{DySCO}_{3}(110)$ is studied as a model system in chapter 3 . The surface termination of $\mathrm{DyScO}_{3}$ after annealing is found to be mixed. Both areas with a $\mathrm{DyO}$ and areas with a $\mathrm{ScO}_{2}$ surface termination are found to be present at the surface. By using a wet-chemical etching procedure, singly $\mathrm{ScO}_{2}$ terminated $\mathrm{DyScO}_{3}$ surface are obtained. No structural evidence for surface reconstructions or displacements of polar $\mathrm{ScO}_{2}$ termination is found. Suggesting that the polar surface is relieved by introducing oxygen vacancies in the topmost $\mathrm{ScO}_{2}$ layer. After annealing of DyScO 3 substrates, the $\mathrm{DyO}$ and $\mathrm{ScO}_{2}$ terminated surface areas showed different degrees of ordering on different samples. In the case of a substrate showing or- 
dered areas of mixed surface termination, these different areas can be observed using an atomic force microscope. For example, when the two areas of different surface termination order along the substrate vicinal steps. Subsequently, an artificial DyO overlayer on singly $\mathrm{ScO}_{2}$ terminated $\mathrm{DyScO}_{3}$ was created by using pulsed laser deposition (PLD). The artificial DyO overlayer adapted its structure to the crystal structure of $\mathrm{DyScO}_{3}$.

Mixed terminated $\mathrm{DyScO}_{3}$ substrates which show ordered areas of both $\mathrm{DyO}$ and $\mathrm{ScO}_{2}$ surface termination are used as templates for the self-organized growth of $\mathrm{SrRuO}_{3}$ in chapter $4 . \mathrm{SrRuO}_{3}$ nanowires form during PLD growth on $\mathrm{DyScO}_{3}$ (110) substrates that show an ordered stripe phase of mixed surface termination. More specifically, $\mathrm{SrRuO}_{3}$ self-organizes on the $\mathrm{ScO}_{2}$ surface termination. The nanowires are epitaxial and are electrically conductive. The amount of deposited material is equal to the volume of the nanowires, indicating all of the deposited material sticks to the surface and diffuses away from the DyO covered surface areas.

This self-organized $\mathrm{SrRuO}_{3}$ growth on mixed terminated $\mathrm{DyScO}_{3}$ substrates is assumed to occur in units of complete perovskite unit-cell blocks. This simplification allows for using existing kinetic Monte Carlo type growth models. The time evolution of the growth of $\mathrm{SrRuO}_{3}$ nanowires is found to be comparable to the time evolution found in experiments, by introducing a difference in activation energy for diffusion on different surface termination areas. Two distinct regions in the time evolution of the nanowire height and width are found. At first, the wires grow mostly in height and the width of the wires equals the width of the surface mixed termination area. However, the wires start to grow in width after a critical nanowire height is reached. This sudden onset of lateral growth is explained using a model similar to the model that is commonly used to describe the onset of second layer nucleation in thin film growth.

Self-organized lateral thin film nanostructures are made using ordered mixed terminated complex oxide substrates. The resulting epitaxial nanowires and nanodots can be used to study size effects in $\mathrm{SrRuO}_{3}$ and in complex oxide heterostructures grown on top of the $\mathrm{SrRuO}_{3}$ nanostructures. For example, by studying the effects of a patterned bottom electrode on the ferroelectric properties of $\mathrm{PbTiO}_{3}$. The ordering of surface termination is also observed on $\mathrm{NdGaO}_{3}$ and $\mathrm{SrTiO}_{3}$ substrates and thus appears to be a general property of perovskite type oxides. In literature the growth kinetics of $\mathrm{SrRuO}_{3}$ on $\mathrm{TiO}_{2}$ and $\mathrm{SrO}$ surface terminations are found to be different. However, the physical mechanisms that drives the sensitivity of $\mathrm{SrRuO}_{3}$ growth kinetics to the surface termination is not wellunderstood.

The comparable crystal structure of the different perovskite crystals allows for creating thin films, where the in-plane lattice of the substrate material is matched to that of the film material, resulting in epitaxially strained thin films. Strain can be used to tune and enhance thin film properties by influencing the crystal structure. In ultra-thin films and nanostructures of only several atomic layers in thickness, the interface layers formed at the substrate-film interface and at the surface account for a substantial part of the material volume and thus contribute 
significantly to the film properties.

In chapter six, the effect is size reduction in ultra-thin films of $\mathrm{SrCuO}_{2}$ is studied. The theoretically predicted structural phase transition in $\mathrm{SrCuO}_{2}$, where one oxygen atom is displaced, is used as a model system for studying thin film structural parameters by $\mathrm{X}$-ray photoelectron diffraction (XPD). Upon reducing the film thickness of $\mathrm{SrCuO}_{2}$ from 9 layer to 3 layer, a structural transition is found. The c-axis of the film as determined by XPD is found to be $\sim 3.4 \AA$ for the 9 layer film and $\sim 3.8 \AA$ for the 3 layer film. This is in accordance with the theoretically predicted structural transition. Moreover, previous measurements on superlattice structures already indicated this structural transition occurs in experiments. In the current work this transition is confirmed to occur in the single-layer thin films as well.

In the final chapter, the effects of size reduction in $\mathrm{SrRuO}_{3}$ thin films are studied. Literature reports show that when the film thickness of $\mathrm{SrRuO}_{3}$ is reduced, the normally conductive films become insulating and are no longer ferromagnetic. However, above four unit-cell layers in thickness, all of the film layers contribute to the magnetic signal, i.e., no dead layer is observed in $\mathrm{SrRuO}_{3}$ thin films. Theoretical calculations relate the onset of conductivity and ferromagnetism in ultra-thin $\mathrm{SrRuO}_{3}$ films to a structural transition from nearly orthorhombic to nearly tetragonal. The magnetic ground state of tetragonal $\mathrm{SrRuO}_{3}$ is calculated to be anti-ferromagnetic. In this thesis, several attempts are made to confirm either the structural or the magnetic transition. Initial experimental evidence for a structural transition is found. The ground state of ultra-thin $\mathrm{SrRuO}_{3}$ films below the critical thickness is found to be paramagnetic.

Theoretical calculations relate the interfacial coupling at the substrate film interface of the oxygen octahedra of $\mathrm{SrRuO}_{3}$ to its structure and magnetic properties. By adding a $\mathrm{SrTiO}_{3}$ capping layer, the ferromagnetic $\mathrm{T}_{\mathrm{C}}$ of $\mathrm{SrRuO}_{3}$ films on $\mathrm{DyScO}_{3}$ substrates is found to be enhanced to nearly bulk values. This enhancement is attributed to the changes in the octahedral tilt and rotation patterns of $\mathrm{SrRuO}_{3}$ induced by the $\mathrm{SrTiO}_{3}$ capping layer. This novel method for enhancing the magnetic properties of $\mathrm{SrRuO}_{3}$ is of great interest for understanding and enhancing the properties of $\mathrm{SrRuO}_{3}$ film with reduced dimensions. 


\section{Samenvatting}

De perovskiet oxide materiaal klasse omvat een veelvoud aan interessante fysische eigenschappen, zoals supergeleiding bij hoge temperatuur en ferromagnetisme bij kamertemperatuur. De vergelijkbare zuurstof structuur en roosterconstantes in zulke $\mathrm{ABO}_{3}$ perovskieten maken het mogelijk om oxide heterostructuren te fabriceren. Hierin kunnen materialen met verschillende intrinsieke eigenschappen worden gecombineerd met als doel de eigenschappen te verbeteren of om nieuwe eigenschappen te vinden. Bij het reduceren van de afmetingen van zulke complexe oxide materialen tot slechts enkele atoomlagen worden veelal eigenschappen gevonden die anders zijn dan de bulk waardes. De drijfveer van het onderzoek naar de aard van deze aangepaste eigenschappen, die zowel intrinsiek als extrinsiek kunnen zijn, is het steeds kleiner maken van elektronica.

Reduceren van afmetingen in oxidische dunne lagen kan in verschillende richtingen. De meest intuitieve vorm van verkleinen is om de dikte terug te brengen, waarbij een tweedimensionale laag wordt gevormd. Het verder terug brengen van het aantal eindige afmetingen resulteert in eendimensionale draden of nuldimensionale spikkels. In dit proefschrift worden dunne lagen gemaakt waarbij de grootte en het aantal vrij te kiezen dimensies wordt beperkt. Door epitaxiale dunne lagen te bestuderen worden intrinsieke effecten van het substraat geïnduceerde spanning en octaëder koppeling verkend. Het eerste deel van dit proefschrift is er op gericht om epitaxiale nanostructuren te maken waarbij de laterale afmetingen worden beperkt tot slechts nul of één vrij te kiezen dimensie. In het laatste deel van dit proefschrift worden twee gevallen besproken van het reduceren van afmetingen in twee dimensionale dunne lagen.

Om nanostructuren te maken is een nieuwe manier verkend om patronen in dunne lagen te maken. $\mathrm{ABO}_{3}$ complexe oxides zijn intrinsiek gelaagd en bestaan uit afwisselende lagen $\mathrm{AO}$ en $\mathrm{BO}_{2}$. In het geval van $\mathrm{DyScO} 3$ (110) hebben deze lagen een tegenovergestelde lading. De precieze structuur van het oppervlak van $\mathrm{DyScO}_{3}$ (110) is bestudeerd als een model systeem in hoofdstuk 3. De oppervlakte terminatie na verwarmen is een mengsel van $\mathrm{DyO}$ and $\mathrm{ScO}_{2}$. Zowel gebieden met een $\mathrm{DyO}$ en gebieden met een $\mathrm{ScO}_{2}$ oppervlakte terminatie zijn gevonden met behulp van oppervlakte analyse technieken. Door gebruik te maken van een chemisch ets proces zijn enkelvoudig $\mathrm{ScO}_{2}$ getermineerde $\mathrm{DyScO}_{3}$ oppervlaktes verkregen. Er zijn geen aanwijzingen gevonden die duiden op oppervlakte reconstructies of verplaatsing van atomen aan het oppervlak van $\mathrm{ScO}_{2}$ getermineerd $\mathrm{DyScO}_{3}$. Dit suggereert dat zuurstof vacatures aan het oppervlak de polaire eigenschap van $\mathrm{DyScO}_{3}$ verhelpen. Om een dubbel getermineerd oppervlakte te verkriigen, worden er samples verhit. $\mathrm{Na}$ het verwarmen van $\mathrm{DyScO}_{3}$ 
zijn op verschillende samples verschillende types van ordering van $\mathrm{DyO}$ en $\mathrm{ScO}_{2}$ zichtbaar. Als het substraat geordende gebieden van beide terminaties heeft, bijvoorbeeld een ordening langs de bestaande stapranden, kunnen deze gebieden gemeten worden. Met behulp van gepulste laser depositie (PLD) is een kunstmatige DyO laag aangebracht op enkelvoudig $\mathrm{ScO}_{2}$ getermineerd $\mathrm{DyScO}_{3}$. Deze kunstmatige laag neemt de kristalstructuur van het substraat over.

$\mathrm{DyScO}_{3}$ substraten met geordende gebieden van zowel $\mathrm{DyO}$ als $\mathrm{ScO}_{2}$ terminatie zijn gebruikt als sjabloon voor groei van $\mathrm{SrRuO}_{3}$ in hoofdstuk 4 . Tijdens het groeien wordt het patroon in de terminatie gevolgd, $\mathrm{SrRuO}_{3}$ nanodraden worden gevormd op $\mathrm{DyScO}_{3}$ substraten die een gestreepte gemengde terminatie hebben. Deze nanodraden vormen zich op de $\mathrm{ScO}_{2}$ getermineerde gebieden van het $\mathrm{DyScO}_{3}$ substraat. De nanodraden zijn epitaxiaal en elektrisch geleidend. De hoeveelheid gedeponeerd materiaal is gelijk aan het volume aan materiaal in de nanodraden. Dit duidt er op dat al het gedeponeerde materiaal hecht aan het oppervlak en via diffusie op de $\mathrm{ScO}_{2}$ getermineerde gebieden komt.

Als aangenomen wordt dat alle processen plaats vinden in complete perovskiet eenheidscel blokken, kan deze vorm van zelforganisatie tijdens $\mathrm{SrRuO}_{3}$ groei op $\mathrm{DyScO}_{3}$ substraten worden beschreven met bestaande groei modellen. Deze versimpeling maakt het mogelijk om bestaande kinetische Monte Carlo type modellen te gebruiken. De gesimuleerde tijdsevolutie van de groei van $\mathrm{SrRuO}_{3}$ nanodraden is vergelijkbaar met de tijdsevolutie die gevonden is in experimenten. Een verschil in activatie energie voor diffusie is geïntroduceerd voor gebieden met verschillende oppervlakte terminatie. In de tijdsevolutie kunnen twee delen worden gedefinieerd. Initieel groeien de draden voornamelijk in de hoogte terwijl de breedte gelijk blijft aan de oorspronkelijke breedte van de gemengde terminatie. Na het bereiken van een kritische hoogte beginnen de draden ook in breedte te groeien. Dit plotse begin van laterale groei is uitgelegd met een model dat vergelijkbaar is met het model dat wordt gebruikt om het begin van nucleatie in de tweede atoomlaag te beschrijven in dunne laag groei.

Door gebruik te maken van gemengde oppervlakte terminatie en zelforganisatie zijn nanostructuren gemaakt in dunne lagen. Deze epitaxiale nanodraden en nanostippen kunnen worden gebruikt om de invloed van afmetingen op de eigenschappen te meten in complexe oxide heterostructuren. Hiermee zijn bijvoorbeeld de effecten van een gepatroneerde elektrode op de ferroelektrische eigenschappen van $\mathrm{PbTiO}_{3}$ bestudeerd. De ordening van oppervlakte terminatie is ook geobserveerd op $\mathrm{SrTiO}_{3}$ and $\mathrm{NdGaO}_{3}$ substraten en lijkt hiermee dus een algemene eigenschap van perovskieten. In de literatuur zijn de kinetische effecten

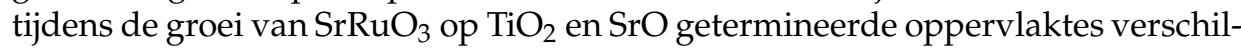
lend bevonden. Echter, het exacte mechanisme dat leid tot de gevoeligheid van $\mathrm{SrRuO}_{3}$ voor de verschillende terminaties is niet bekend.

Door de vergelijkbare kristalstructuur van de verschillende perovskiet kristallen kunnen dunne lagen worden gemaakt waarbij de rooster constanten van het substraat materiaal gelijk is aan die van het dunne laag materiaal. Dit resulteert in epitaxiale lagen die onder spanning staan. Door invloed van deze spanning verandert de structuur van de lagen en dus de eigenschappen. De hoeveelheid 
spanning die wordt geïnduceerd door het substraat is afhankelijk van deze keuze van het substraat materiaal. De eigenschappen van de laag kunnen dus worden bewerkt door de keuze van het substraat materiaal. In het geval van ultradunne lagen van slechts enkele atoomlagen dik, vormt het grensvlak tussen de laag en het substraat en de laag en vacuüm een substantieel deel van het volume aan materiaal. Deze grensvlakken spelen dus een belangrijke rol bij het bepalen van de eigenschappen van zulke ultradunne lagen.

In hoofdstuk zes worden de effecten van het reduceren van de laagdikte in ultradunne $\mathrm{SrCuO}_{2}$ lagen bestudeerd. De theoretisch voorspelde faseovergang in $\mathrm{SrCuO}_{2}$ waarbij een zuurstof atoom van plaats veranderd wordt gebruikt als een model systeem voor het bestuderen van de structurele eigenschappen van dunne lagen met behulp van X-ray photoelectron diffractie (XPD). Een structuur verandering is gemeten als de laagdikte wordt teruggebracht van 9 naar 3 lagen. De c-as van de laag is bepaald op $3.4 \AA$ in het geval van 9 lagen en $3.8 \AA$ in het geval van 3 lagen $\mathrm{SrCuO}_{2}$. Dit komt overeen met de theoretische voorspelde faseovergang. Eerdere metingen op supperlattice structuren hebben bovendien al aangeven dat deze faseovergang plaats kan vinden. In dit proefschrift is de faseovergang bevestigd voor enkelvoudige dunne lagen.

In het laatste hoofdstuk wordt het effect van het reduceren van de laagdikte van $\mathrm{SrRuO}_{3}$ dunne lagen bestudeerd. Vanuit de literatuur is bekend dat als de laagdikte van $\mathrm{SrRuO}_{3}$ wordt teruggebracht dat de normaal elektrisch geleidende lagen isolerend worden en niet meer ferromagnetisch zijn. Boven de vier atoomlagen in dikte dragen alle lagen bij aan de magnetische eigenschappen; er is geen dode laag in $\mathrm{SrRuO}_{3}$ dunne lagen. Theoretische berekeningen verbinden de plotse overgang van isolerend naar geleidend en ferromagnetisch aan een verandering van structuur van bijna orthorombisch naar bijna tetragonaal. Hierbij is voorspeld dat de magnetische grondtoestand antiferromagnetisch is. In dit proefschrift worden verschillende poging gedaan om de structurele en/of de magnetische overgang te bevestigen. Initiële experimentele resultaten geven aan dat er een verandering van structuur plaats vindt. Uit metingen van de magnetische eigenschappen blijkt dat de grond toestand van ultradunne $\mathrm{SrRuO}_{3}$ lagen dunner dat de kritische dikte paramagnetisch is.

Theoretische berekeningen verbinden de koppeling van de zuurstof octaëders tussen het substraat en de dunne laag $\mathrm{SrRuO}_{3}$ aan de structuur en magnetische eigenschappen van $\mathrm{SrRuO}_{3}$. Door een toplaag van $\mathrm{SrTiO}_{3}$ toe te voegen wordt de ferromagnetische overgangstemperatuur verhoogd tot bijna bulk waardes. Deze verhoging is toegewezen aan de verandering van zuurstof octaëder verbuigingen en verdraaiingen in $\mathrm{SrRuO}_{3}$ die worden geïnduceerd door de $\mathrm{SrTiO}_{3}$ toplaag. Deze nieuwe methode om de magnetische eigenschappen van $\mathrm{SrRuO}_{3}$ te modificeren is interessant voor een beter begrip en voor het verbeteren van de eigenschappen van $\mathrm{SrRuO}_{3}$ dunne lagen met gereduceerde afmetingen. 


\section{Dankwoord}

De afgelopen vier jaar heb ik met veel plezier aan de totstandkoming van dit proefschrift gewerkt. Het gedane onderzoek dat geleid heeft tot de afronden van mijn boekje is mede mogelijk gemaakt door de steun van anderen. De inhoud is gedeeltelijk tot stand gekomen middels diverse samenwerkingen. Bij dezen wil ik iedereen die een rol heeft gespeeld bij mijn promotie graag bedanken.

Allereerst wil ik mijn promotor en assistent-promotor bedanken voor de gegeven kans en mogelijkheden om bij IMS onderzoek te doen. Beide ontzettend bedankt voor alle interessante discussies, die mij hebben geholpen om mijn onderzoek richting te geven. Gertjan, als dagelijks begeleider heeft jou enthousiasme mij sterk gemotiveerd. Guus, ik waardeer bij jou in het bijzonder de directe manier van communiceren en de duidelijke adviezen die je gaf op basis van jou rijke ervaring. Daarnaast wil ik Dave graag bedanken voor zijn ondersteuning, in het bijzonder in het begin van mijn promotie traject. Dave, jou passie voor de nanotechnologie en je eindeloze nieuwsgierigheid hebben op mij altijd een bijzonder enthousiasmerende werking gehad.

Veel van mijn onderzoek was niet mogelijk geweest zonder samenwerking met collega's, zowel bij de vakgroep IMS als daar buiten. Binnen de vakgroep wil ik graag Josée, David, Nirupam, Brian, Peter B, Peter dV, Rik, Debakanta, Evert, Mark, Adré en de mede-TOPpers Wouter en Maarten bedanken voor hun bijdrages aan mijn onderzoek. Outside of the IMS group, I would especially like to thank my collaborators: Arturas at Stanford University, Lior and Noam at Bar Ilan University, Zhicheng at Vienna University of Technology, Gabriella at SPINCNR in Napoli, Jing and Sean at University of California Irvine, Ricardo and Jo at University of Antwerp and Giuseppe at ESRF in Grenoble. Binnen Nederland wil ik graag Paul en Elias van de Radboud Universiteit Nijmegen en Sybolt, Xiao en Joost van de Universiteit Twente bedanken voor hun samenwerking. Daarnaast heb ik de input van mijn Master en Bachelor studenten Chris en Daniël erg gewaardeerd.

Het werk in het COMAT lab heeft mij altijd veel plezier gegeven. Voor de hulp bij reparaties en onderhoud en voor de gezellige COMAT diners en uitjes wil ik graag alle medegebruikers bedanken: Jeroen, Joost, Hans, Josée, Andreas, Brian, Chris, Rik, Werner, Michelle, David en Tom. In het bijzonder wil ik ook de technici Henk, Arjen, Gerrit en Dominic van IMS en Frank en Dick van ICE bedanken voor hun zeer praktische hulp bij mijn onderzoek. Marion en José, bedankt voor jullie hulp bij de problemen en vragen van mij buiten het lab.

De tijd in de IMS vakgroep heb ik als erg gezellig en leuk ervaren. Naast de interessante discussies bij werkbesprekingen of aan de koffietafel waren vooral ook 
de evenementen en uitjes erg leuk, zoals de kerstlunches en de studiereis naar Oxford. Bedankt, Brian, Nirupam, Josée, Hans, Jeroen, Xin, Ruud, Alim, Rik, Tom, Werner, Michelle, Peter B, Peter dV, Oktay, David, Kurt, Kenan, Anirban, Maarten, Wouter, Rogier, Huiyu, Sjoerd, Pablo, Hajo, Petra, Roy, Bahruz, Maaike, Muhammed, Joska, Gerard, Michiel, Eddy, Anuj, Evert, Zhaoliang, Minh, Debakanta, Dominic, Gerrit, Henk, Arjen, José, Marion, Guus, Gertjan, Mark, André, Dave, Jos, Bernard, Gerwin, Ben, Tjeerd, Anthony, Willem, Suresh, Wolter, Tülin, Tomasz, Nicolas, Jaap, Matjaz, Matthijn, Herman, Ole en Ronald.

In de periode dat ik aan mijn promotie werkte heb ik met veel plezier badminton gespeeld bij D.B.V. DIOK. Ook heb ik de nodige afleiding gevonden tijdens de gezellige diners met vrienden. Ik heb grote waardering voor iedereen die met veel interesse probeerde te begrijpen wat ik precies deed in het nanolab. Dank ook aan mijn paranimfen Rick en Rikkert. Rick bedankt voor de vele leuke badmintonwedstrijden samen en Rikkert bedankt voor afleiding met spelletjes en puzzels.

Tot slot wil ik graag mijn familie bedanken. Bijzonder dankbaar ben ik voor de eindloze en onvoorwaardelijke steun van mijn ouders en broertje gedurende mijn gehele opleiding. Hoewel mijn vader de afronding van mijn promotie helaas niet mee kan maken, vind ik rust in de gedachte dat hij erg trost op mij zou zijn. Ontzettend dankbaar ben ik voor de steun van mijn lieve vrouw. Marloes, jouw onuitputtelijke geduld en realistische wereldblik hebben mij heel sterk geholpen de afgelopen vier jaar. Jouw liefdevolle ondersteuning geeft mij de mogelijkheid om het beste uit mezelf te halen. Een laatste woord van dank gaat uit naar mijn dochter Elise. Gedurende de laatste zeventien maanden van mijn promotie en het schrijven van mijn proefschrift hebben jou lach en stralende ogen mij vrolijk gehouden en gemotiveerd.

Bouwe Kuiper

Enschede, januari 2014 